\title{
Monte Carlo-based analysis of the effect of positional and thematic uncertainties in thematic maps on biodiversity model coefficients
}

by

Patrick Kirby

A thesis submitted to the

Faculty of Graduate and Postdoctoral Affairs

in partial fulfillment of the requirements for the degree of

\author{
Master of Science \\ in \\ Geography
}

Department of Geography and Environmental Studies,

Carleton University,

Ottawa, Ontario

(C) 2015

Patrick Kirby 


\begin{abstract}
When modelling, considering the influence of all forms of uncertainty is important. This study used Monte Carlo simulation approaches to quantify the influence of positional and thematic uncertainties in landscape maps on metrics and model coefficients based on these maps. First, a brief comparison of simulation approaches, differing in their consideration of spatially correlated thematic error within agricultural fields, was conducted. Results helped to identify an approach under which the output distributions best-represented reference metrics. Second, the influence of both positional and thematic uncertainties on model coefficients were quantified and compared to already-considered forms of uncertainty. Three simulation approaches, differing in how they consider spatially correlated thematic error between fields, were used. Results suggest that the influence of positional and thematic uncertainties was lower. The demonstrated simulation approaches may be useful to studies in similar landscapes, where local reference data are not available, for updating coefficient confidence intervals.
\end{abstract}




\section{Acknowledgements}

I would like to give many thanks to my thesis supervisor, Dr. Scott Mitchell, for the support and guidance that he provided over the past few years. Additional thanks to committee member Dr. Murray Richardson, for thesis advice and a modelling background.

Thank you, Dr. Lenore Fahrig and Dr. Jude Girard, for developing the models that were used in this study. Additional thanks to Dr. Girard for explaining these models and answering many questions related to the Farmland Biodiversity Project. Thank you, Dr. Dennis Duro, for answering questions related to the same project, for providing advice, and for providing very helpful explanations related to image analysis. Thank you, Dr. Adam Smith, for a very helpful discussion regarding modelling and model averaging. Thank you, Dan Bert, for assistance with technical issues in the lab. Thank you, Jessica van den Berg, for providing a detailed crop classification study in the area, as well as providing data for pilot projects. Additional thanks to Dr. Doug King, Dr. Kathryn Lindsay, Dr. Scott Mitchell, Dr. Jon Pasher, Dr. Lutz Tischendorf, and many others involved in the Farmland Biodiversity Project.

Thanks to all of the faculty and staff in Carleton's Department of Geography and Environmental Studies that have provided knowledge and support over the past several years. Many thanks to Dr. Andrew Davidson, Dr. Doug King, Dr. Scott Mitchell, and Dan Patterson for the background in remote sensing and GIS that they provided over the years. Thank you, Dr. Gita Ljubicic, for the background in thesis writing. Thank you, Judy Eddy, Natalie Pressburger, and Erin Johnston, for the administrative guidance and support over the years. 
Thanks to the National Wildlife Research Centre, Environment Canada, Carleton University, the Government of Ontario, Mrs. Elizabeth Fleming, Auto-Carto Six, the Canadian Association of Geographers GIScience study group, and ESRI Canada for funding and support. Thank you to the Earth Observation Team of the Science and Technology Branch at Agriculture and Agri-Food Canada (via Dr. Andrew Davidson) for supplemental ground reference data. Thank you to the Natural Sciences and Engineering Research Council of Canada for funding of the Farmland Biodiversity Project.

Finally, many thanks to friends and family for all of the support. Thank you Mom, Bob, Dad, Julie, Dan, Cassi, Lib, and Jill. It is always a pleasure spending time with you all, and I could not have done it without you. 


\section{Table of Contents}

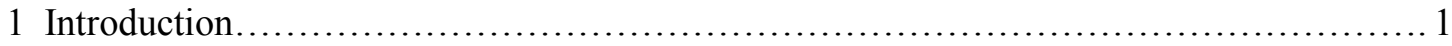

1.1 Uncertainty, uncertainty propagation, and its importance........................ 2

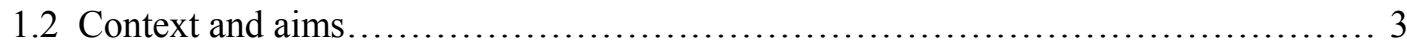

1.3 Research questions.................................................... 5

1.4 Thesis Structure.................................................................. 5

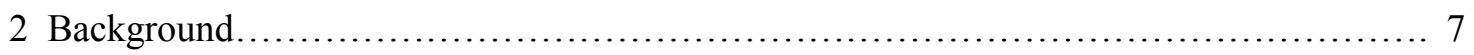

2.1 Biodiversity strategies in Canada and Ontario................................. 7

2.2 Biodiversity and agricultural landscapes................................... 7

2.3 Characterizing landscapes with remotely sensed imagery....................... 9

2.3.1 Image classification................................................. 10

2.3.1.1 Maximum likelihood and Bayesian classifiers........................... 11

2.3.1.2 Classification trees and Random Forests.............................. 12

2.3.1.3 Hard and soft classifications......................................... 14

2.3.2 Digitizing and polygon representation................................... 16

2.3 .3 Object-based image analysis........................................... 17

2.4 Error, accuracy, and uncertainty in spatial data................................. 17

2.4.1 Error and uncertainty in thematic maps................................... 18

2.4.2 Error and uncertainty in point and linear features..........................20

2.5 Uncertainty analysis and Monte Carlo methods................................ 21

2.5.1 Monte Carlo simulation................................................... 22

2.5.2 Sequential indicator simulation............................................. 24

2.5.3 Field-based Monte Carlo simulation.........................................25

2.6 Farmland Biodiversity Project.............................................. 26

2.6.1 Modelling............................................................... 29

\section{Continued on next page}




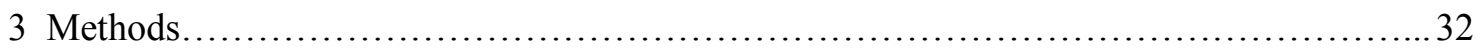

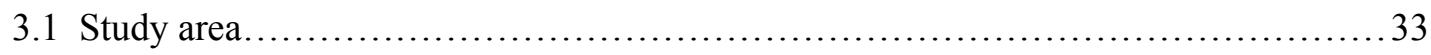

3.1 .12011 Ontario growing season........................................ 35

3.1 .22012 Ontario growing season......................................... 35

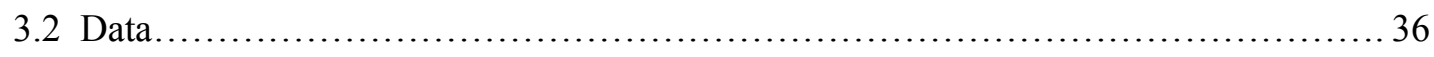

3.2.1 Data from the Farmland Biodiversity Project................................ 36

3.2.2 Data from Agriculture and Agri-Food Canada.................................... 39

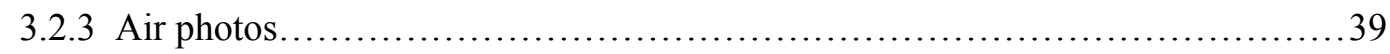

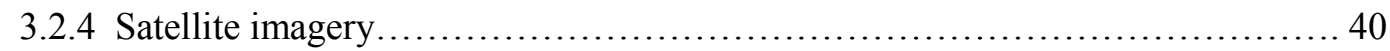

3.3 Simulation of the effect of thematic uncertainty on SHDI...........................43

3.3.1 Site selection............................................................. 44

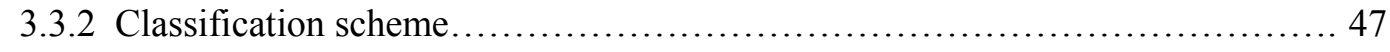

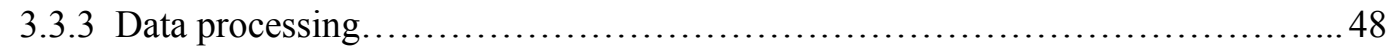

3.3.4 Classifier training areas.................................................... 50

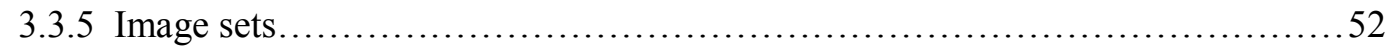

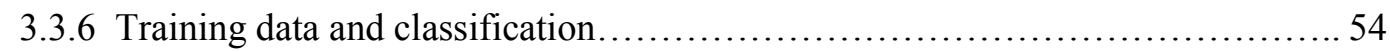

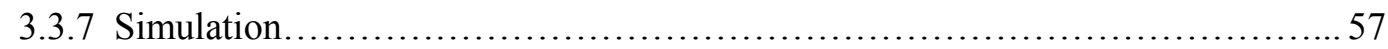

3.4 Effects of positional and thematic uncertainties on the model coefficients.............59

3.4.1 Positional uncertainty estimation: Vertex position uncertainty..................6 61

3.4.2 Positional uncertainty estimation: Boundary interpretation uncertainty...........64

3.4.2.1 Merge and split cases.......................................... 66

3.4.2.2 Agricultural versus non-agricultural boundary cases..................... 69

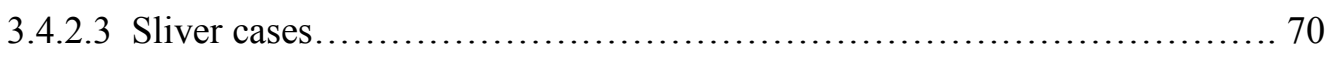

3.4.3 Thematic uncertainty estimation..................................... 70

3.4.3.1 Thematic Mapper imagery processing ............................. 71

3.4.3.2 Classification scheme and training samples......................... 72

3.4.3.3 Classification................................................... 74

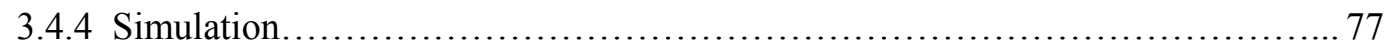

3.4.4.1 Realization generation and landscape metric calculation.................. 77

3.4.4.2 Alternate, grouped simulation approaches.......................... 79

3.4.4.3 Extension of simulation through model averaging ...................... 82

\section{Continued on next page}




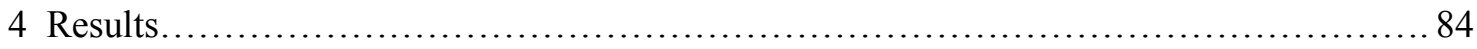

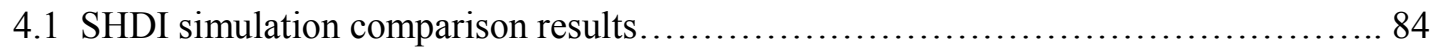

4.1.1 Probabilities of class membership (soft classification) .......................84

4.1 .2 Normalized entropy................................................ 90

4.1.3 Accuracy of the hardened classifications................................ 91

4.1 .4 Landscape realizations............................................. 93

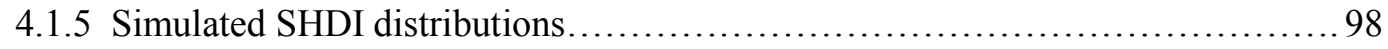

4.1.5.1 Simulation convergence......................................... 104

4.2 Effects of positional and thematic uncertainties on the biodiversity models............ 106

4.2.1 Image classification..................................................... 107

4.2.1.1 Accuracy of the hardened classification............................. 107

4.2.1.2 Normalized entropy........................................... 109

4.2.1.3 Second and third-most likely classes of incorrectly classified fields......... 111

4.2 .2 LC map realizations.................................................. 113

4.2.2.1 Alternate boundaries and thematic classes............................ 114

4.2.2.2 Alternate vertex positions.......................................... 116

4.2.3 Simulated metrics under independent field and grouped approaches..............119

4.2.3.1 Landscape metrics under independent field simulation.....................120

4.2.3.2 Simulated SHDI offset and grouped simulation....................... 123

4.2.4 Simulated model coefficients........................................... 126

4.2.4.1 Offset between original and hard coefficients........................ 128

4.2.4.2 Offset between hard and simulated coefficients......................... 128

4.2.4.3 Positional and thematic uncertainties versus the considered uncertainties....131

4.2.4.4 Convergence of simulated coefficients and variance estimates...............133

\section{Continued on next page}




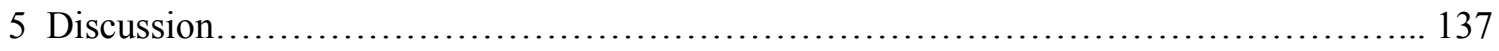

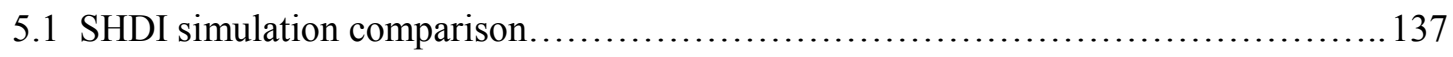

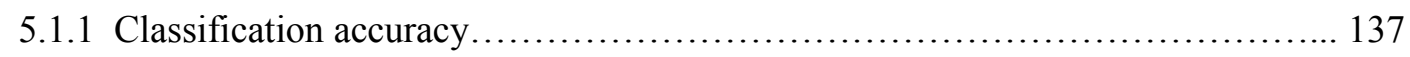

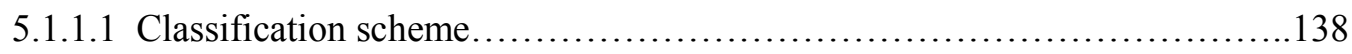

5.1.1.2 Training and validation data limitations............................. 139

5.1 .1 .3 Resolution and classifier........................................ 141

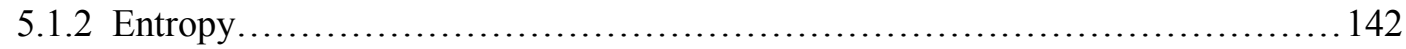

5.1 .3 Structure preservation................................................ 143

5.1 .4 Simulated SHDI distributions......................................... 145

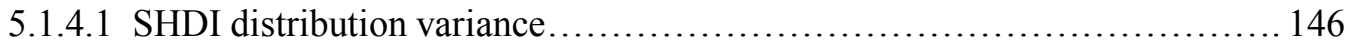

5.1.4.2 SHDI offset........................................................ 147

5.2 Effects of positional and thematic uncertainties on the biodiversity models............ 149

5.2.1 Image classification and thematic uncertainty........................... 149

5.2.1.1 Classification accuracy.......................................... 149

5.2.1.2 Entropy and class probability estimates.............................. 151

5.2 .2 Positional uncertainty................................................... 153

5.2.2.1 Vertex position uncertainty estimates............................... 153

5.2.2.2 Boundary interpretation uncertainty estimates......................... 155

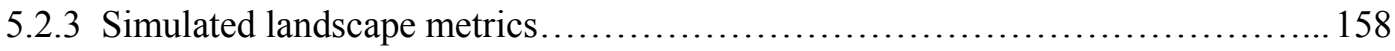

5.2.4 Model averaged coefficients.......................................... 160

5.2.4.1 Coefficient offset between original and hard coefficients................. 161

5.2.4.2 Coefficient offset between hard and simulated coefficients.................. 162

5.2.4.3 Variance comparison........................................... 163

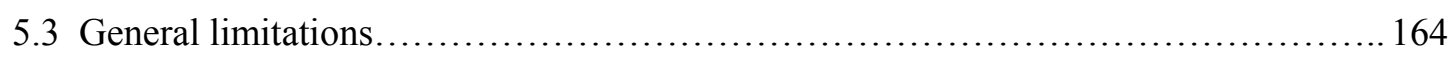

5.4 Further research........................................................ 167

5.4.1 Improvement of positional and thematic uncertainty estimates............. 167

5.4 .2 Alternate simulation approaches................................... 169

5.4.3 Alternate metrics and modelling approaches.......................... 170

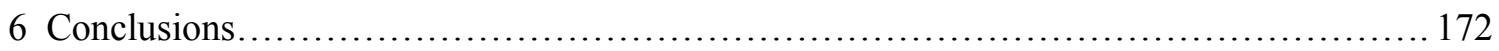

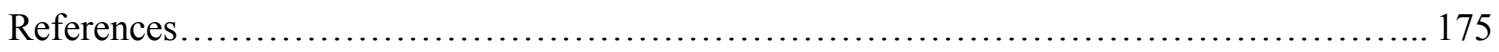




\section{Figures}

Figure 2.1 Illustrated examples of inverse and discrete inverse transform sampling......... 23

Figure 3.1 Distribution of the study landscapes throughout eastern Ontario................. 34

Figure 3.2 Natural colour composite TM images of the study area....................... 42

Figure 3.3 Haze image with numbered QB image extents............................... 45

Figure 3.4 Examples of soybean fields from QB images.............................. 46

Figure 3.5 Field boundary trimming ............................................. 49

Figure 3.6 Distribution of training areas [limited extent classification] .................... 51

Figure 3.7 Number of TM training samples by class [limited extent classification] ......... 52

Figure 3.8 Feature selection for the multi-date TM image set............................ 53

Figure 3.9 Class membership probability raster processing........................... 57

Figure 3.10 Example of positional uncertainties.................................. 60

Figure 3.11 Example of vertex omissions from the vertex position uncertainty estimation..... 63

Figure 3.12 Quantile-quantile plots: vertex position uncertainty vs. normal distribution...... 63

Figure 3.13 Examples of local estimates of boundary interpretation uncertainty.............. 66

Figure 3.14 Examples of the results of three Canny edge detector runs.................... 68

Figure 3.15 Locations of the year-2011 landscape centers relative to the cloud mask.......... 72

Figure 3.16 Number of training samples, by class [year-2011 study area classification] ...... 73

Figure 3.17 Illustrated example of the values retained from model averaging simulation..... 83

Figure 4.1 Posterior probabilities of class membership from single date TM classification... 86

Figure 4.2 Posterior probabilities of class membership from multi-date TM classification... 87

Figure 4.3 Posterior probabilities of class membership from QB classification.............. 88

Figure 4.4 Hardened classifications and realization examples, single date TM classification. 94

Figure 4.5 Hardened classifications and realization examples, multi-date TM classification..95

Figure 4.6 Hardened classifications and realization examples, QB classification............. 96

Figure 4.7 Simulated SHDI distributions ........................................... 99

Figure 4.8 Cumulative SHDI variance versus the number of realizations.................... 105

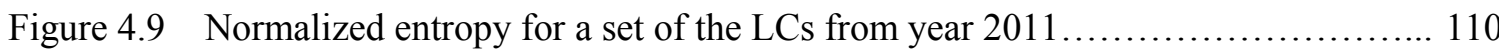

Figure 4.10 Examples of realizations of alternate LC map composition and configuration..... 115

Figure 4.11 Ten realizations of field boundary vertex positions.......................... 117

Figure 4.12 Example of the difference in shape preservation for simulated vertices of fields.. 118

Figure 4.13 Comparison plots for simulated versus re-digitized field boundaries............ 119

Figure 4.14 Distributions of simulated P.AG metrics............................... 120

Figure 4.15 Distributions of simulated MFS metrics................................ 121

Figure 4.16 Distributions of simulated SHDI metrics................................. 122

Figure 4.17 Hard LC maps for which there was an offset between hard and simulated SHDI. 124

Figure 4.18 Offsets in SHDI metrics under different simulation approaches................ 125

Figure 4.19 Hard and simulated regression coefficients and confidence intervals............ 127

Figure 4.20 SHDI coefficients under different simulation approaches.................... 129

Figure 4.21 Offsets in SHDI coefficient under different simulation approaches............. 129

Figure 4.22 Z-scores for original SHDI coefficients relative to simulated coefficients........ 130

Figure 4.23 Cumulative variance in simulated coefficient versus number of realizations..... 135

Figure 4.24 Cumulative mean in simulated SHDI coefficient versus number of realizations... 136 


\section{Tables}

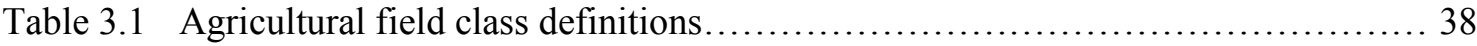

Table 3.2 QuickBird and Thematic Mapper spectral characteristics..................... 40

Table 3.3 QB image details with landscape information................................. 47

Table 3.4 Polygon shrinking distance for area to perimeter ratio intervals................. 76

Table 3.5 Summary table of simulation approaches................................ 81

Table 4.1 Mean normalized entropy for landscapes and [limited extent] classifications...... 90

Table 4.2 Mean normalized entropy by class [limited extent classifications] ............... 91

Table 4.3 Accuracies for the per-field and per-field [limited extent] classifications........... 92

Table 4.4 Variance in SHDI by classification, simulation approach, and landscape........... 100

Table 4.5 Z-scores for reference SHDI relative to the simulated SHDI distributions..........103

Table 4.6 Accuracies for the hardened classifications [year-2011 study area classification].. 108

Table 4.7 Error matrix for the hardened classification [year-2011 study area classification].. 108

Table 4.8 Area weighted mean normalized entropy values by ground referenced class...... 111

Table 4.9 Incorrectly classified fields that have a correct second or third most-likely class... 112

Table 4.10 Proportion of variance: simulated variance vs. model averaging estimate....

\section{Appendices}

Appendix A: Example of independent pixel-based versus field-based simulation

Appendix B: Spectral characteristics of the training data for the limited extent classifications

Appendix C: Transformed divergence for the limited extent classifications

Appendix D: Examples of error and uncertainty in the reference field boundaries

Appendix E: Spectral characteristics and spatial distribution of the training samples for the 2011 study area crop classification

Appendix F: Additional classification entropy and accuracy information [limited extent classifications]

Appendix G: Examples of poorly classified fields with uncertain ground referencing [study area classification]

Appendix H: Hard and simulated biodiversity model coefficients for all diversity types (including abundance)

Appendix I: Uncertainty variance ratios for all models and simulation approaches

Appendix J: Convergence plots across different simulation approaches

Appendix K: Simulated landscape metrics for all LCs

Appendix L: Moran's I statistics

Appendix M: Model summaries 


\section{Table of acronyms and short forms}

\begin{tabular}{|c|c|}
\hline $\begin{array}{l}\text { Acronym/ } \\
\text { short form }\end{array}$ & Description \\
\hline AAFC & Agriculture and Agri-Food Canada \\
\hline $\mathrm{AICc}$ & second-order bias corrected Akaike's Information Criterion \\
\hline $\mathrm{CDF}$ & cumulative density function \\
\hline$E_{\mathrm{n}}$ & normalized entropy \\
\hline GIS & geographical information systems \\
\hline $\mathrm{LC}$ & $\begin{array}{l}1 \mathrm{~km} \text { by } 1 \mathrm{~km} \text { landscape center. While this scale can still be considered a } \\
\text { landscape, the acronym is used to distinguish between the full } 3 \mathrm{~km} \text { by } 3 \mathrm{~km} \\
\text { extents (analyzed in Section } 3.3 \text { ) and the } 1 \mathrm{~km} \text { by } 1 \mathrm{~km} \text { extents (analyzed in } \\
\text { Section 3.4). }\end{array}$ \\
\hline MC & Monte Carlo \\
\hline MFS & mean agricultural field size (landscape metric; predictor variable) \\
\hline NDVI & normalized difference vegetation index \\
\hline OBIA & object-based image analysis \\
\hline OMAFRA & Ontario Ministry of Agriculture Food and Rural Affairs \\
\hline \multirow[t]{2}{*}{ P.AG } & proportion of land in agricultural production \\
\hline & (landscape metric; predictor variable) \\
\hline PDF & probability density function \\
\hline PRN & pseudo-random number \\
\hline QB & QuickBird \\
\hline RMSE & root mean square error \\
\hline RS & remotely sensed \\
\hline SAR & synthetic aperture radar \\
\hline SHDI & Shannon diversity index (landscape metric; predictor variable) \\
\hline SIS & sequential indicator simulation \\
\hline TM & Thematic Mapper (Landsat-5 sensor) \\
\hline
\end{tabular}

\section{Table continued on next page}




\section{Table of acronyms and short forms (continued)}

\begin{tabular}{|c|c|}
\hline $\begin{array}{l}\text { Acronym/ } \\
\text { short form }\end{array}$ & Description \\
\hline$\widehat{\bar{\Theta}}_{\text {hard }}$ & $\begin{array}{l}\text { model averaged coefficient estimates based on the hard dataset (reference } \\
\text { field boundaries, with year-2011 crop types based on hardened image } \\
\text { classification) }\end{array}$ \\
\hline$\widehat{\operatorname{se}}\left(\widehat{\bar{\Theta}}_{\text {hard }}\right)$ & $\begin{array}{l}\text { unconditional model averaging standard error estimates associated with } \widehat{\widehat{\Theta}}_{\text {hard }} \text {; } \\
\text { has a corresponding variance estimate, } \widehat{\operatorname{var}}\left(\widehat{\widehat{\Theta}}_{\text {hard }}\right)\end{array}$ \\
\hline$\widehat{\bar{\Theta}}_{\text {sim }}$ & $\begin{array}{l}\text { model averaged coefficient estimates (for either P.AG, MFS, or SHDI), } \\
\text { averaged across all realizations }\end{array}$ \\
\hline$\widehat{\operatorname{se}}\left(\widehat{\bar{\Theta}}_{\text {sim }}\right)$ & $\begin{array}{l}\text { unconditional model averaging standard error estimates associated with } \widehat{\bar{\Theta}}_{\text {sim }} \text {, } \\
\text { pooled across all realizations; has a corresponding variance estimate, } \\
\widehat{\operatorname{var}}_{\left(\hat{\mathrm{\theta}}_{\mathrm{sim}}\right)}\end{array}$ \\
\hline$\sigma_{\text {sim }}$ & $\begin{array}{l}\text { standard deviation in } \widehat{\bar{\Theta}}_{\text {sim }} \text {, across all realizations; has a corresponding } \\
\text { variance, } \sigma_{\text {sim }}^{2}\end{array}$ \\
\hline$\widehat{\bar{\beta}}_{\text {P.AG }}$ & model averaged coefficient for the P.AG variable \\
\hline$\widehat{\bar{\beta}}_{\mathrm{MFS}}$ & model averaged coefficient for the MFS variable \\
\hline$\widehat{\bar{\beta}}_{\text {SHDI }}$ & model averaged coefficient for the SHDI variable \\
\hline
\end{tabular}




\section{Introduction}

Ecology involves the study of the interactions and processes amongst living organisms and between living organisms and their environment (Stine \& Hunsaker, 2001). Landscape ecologists are particularly interested in how landscapes, heterogeneous land areas of interacting ecosystems, are structured, function, and change (Forman \& Godron, 1986). Since these landscapes and the processes within them can be quite complex, a large amount of spatial data often must be acquired, managed, and analyzed for their study (Haines-Young, Green, \& Cousins, 1993; Eastman, 2001). Thus, due to their potential for managing complex spatial data, geographical information systems (GIS) have played an increasing role in landscape ecology since the late 1980's and are now the standard tool for handling spatial data associated with landscapes (Haines-Young et al. 1993; Eastman, 2001; Green, Klomp, Rimmington, \& Sadedin, 2006). In addition, the use of remotely sensed (RS) imagery has become widespread in ecology (Friedl, McGwire, \& McIver, 2001). While seeing use for well over 100 years in the form of aerial photographs, RS products have become more accessible, versatile, and precise, allowing users to characterize more elements of the earth's surface over vast extents (Jensen, 2000; Lillesand, Kiefer, \& Chipman, 2004). GIS and RS data have helped to open new areas of ecological research (Stine \& Hunsaker, 2001). Both can play a role in policy, planning, or decision-support in the ecological context (Eastman, 2001; Stine \& Hunsaker, 2001). 


\subsection{Uncertainty, uncertainty propagation, and its importance}

Data and analyses required to study landscape characteristics and processes include potential error, uncertainty, and uncertainty propagation. When performing these analyses, an understanding of the uncertainties and their propagation is important, as they can inform us on the probability of error (Dungan, 2002). Scientists, for example, are interested in the risk of error when accepting or rejecting a hypothesis. Similarly, decision-makers often rely on risk assessment to quantify the chances of making a good or bad decision, and how to best manage the risk inherent in any decision (Thompson \& Graham, 1996). With scientific assessments often informing decision-makers, the Millennium Ecosystem Assessment's report on ecosystems and human well-being states that "it is essential that any such assessments be accompanied by an accurate and understandable description of underlying assumptions, associated uncertainties and the implications of the uncertainties for the potential outcomes of decisions being made" (Corvalan et al. 2005, p. 47).

The propagation of model input uncertainties can be difficult to quantify analytically, as algebraic complexity tends to increase with model complexity (Morgan, Henrion, \& Small, 1990). Alternatively, Monte Carlo (MC) simulation methods are black-box, stochastic simulation techniques that can be used to quantify uncertainty propagation without an in-depth understanding of how the model functions (Crosetto, Ruiz, \& Crippa, 2001). MC simulation is one of the most commonly used approaches to uncertainty propagation analyses, including those of GIS-based products (Canters, 1997; Brown \& Heuvelink, 2007). It involves repeatedly sampling probabilistic representations 
of model inputs to generate multiple error-sensitized input samples, or realizations, and repeatedly applying the model function or algorithm to each set of sensitized inputs, ultimately generating a distribution of possible model outputs (Crosetto, Tarantola, \& Saltelli, 2000; Crosetto et al. 2001). The characteristics of the output distributions, representative of propagated input uncertainties, are then analyzed (Crosetto et al. 2000; Crosetto et al. 2001).

Landscape ecology studies often use spatial data obtained from thematic land cover maps. These data exist in three dimensions: (1) spatial, (2) thematic, and (3) temporal (Bhatta, 2011). With the temporal dimension assumed constant in many studies, geostatistical MC-based simulation methods are often used to consider uncertainty in the spatial and thematic dimensions. However, they can rely on local reference data and many not be ideal for landscapes with a well-defined spatial structure (e.g. thematic maps of crop type at the farm scale) (Gebbers \& de Bruin, 2010; Caers, 2011).

\subsection{Context and aims}

The research presented in this thesis is situated within a larger project being conducted at Carleton University's Geomatics and Landscape Ecology Laboratory. The Farmland Biodiversity Project seeks to develop a better understanding of how agricultural landscapes can be structured, in terms of composition or configuration, so that biodiversity can be enhanced without removing land from agricultural production (Fahrig et al. 2011). It has involved modelling the relationships between biodiversity and metrics of landscape composition and configuration, for agricultural landscapes in 
eastern Ontario. While uncertainties associated with the sample and model selection were considered, uncertainties associated with measurement error in the landscape metrics were not addressed in depth, as the landscape maps had been ground referenced (Anderson, 2008; Fahrig et al. 2015).

This thesis explores the use of MC methods that are simple compared to many geostatistical approaches, and do not rely on local reference data. The aim is twofold. First, by demonstrating a MC approach to quantifying positional and thematic uncertainty propagation in landscapes with a well-defined spatial structure, without reliance on local reference data, it aims to contribute to the knowledge base surrounding uncertainty propagation analysis for landscapes of this nature. Second, it aims to contribute to the Farmland Biodiversity Project by providing estimates of the uncertainties in model coefficients that can be attributed to positional and thematic uncertainties in the landscape maps, under the scenario that not all of these maps had been ground referenced, instead based on image digitizing and classification.

Since the maps used in the Farmland Biodiversity Project models were ground referenced, the scenario adopted by this thesis deviates from the data collection methods of that project. However, the use of image digitizing and classification could be useful, cost-effective tools for map production, if the dataset were to be spatially or temporally expanded.

The term 'field' is used frequently throughout this thesis, referring to agricultural fields within the landscapes. However, as noted by Canters, 1997, field-based simulation approaches can apply to contiguous groups of pixels that share a thematic class, 
regardless of the land cover or use, as long as the spatial transitions in class are abrupt, rather than gradual.

\subsection{Research questions}

To accomplished the aims stated above, the following three research questions are posed:

(1) For a set of landscape maps that were produced through RS imagery digitizing and classification, how do MC simulation methods with different assumptions regarding within-field spatial autocorrelation differ in terms of bias, variance, and structure preservation when used to quantify the influence of thematic uncertainty on a crop diversity metric?

(2) For the landscape maps, can MC simulation methods that don't rely on local reference data be applied to quantify the effect of positional and thematic uncertainties on model coefficients?

(3) For a set of eastern Ontario biodiversity models, how does the influence of positional and thematic uncertainties on model coefficients compare to already-considered uncertainty estimates from the modelling approach?

\subsection{Thesis Structure}

There are six sections to this thesis. Section 1 provides a brief introduction to some of the key concepts of the thesis, describes the context and aims of the thesis, and presents the research questions. Section 2 provides more-detailed background information on policy, concepts, and analysis techniques related to this thesis. Section 3 describes the methods employed by the thesis study. The study area and available data 
are first described (Sections 3.1 and 3.2, respectively), as this information is important for the understanding of all subsequent methodology sections. Methods related to a brief comparison of simulation approaches for thematic uncertainty quantification, addressing research question 1, are then described (Section 3.3). This is followed by a description of those methods related to a broader uncertainty analysis considering both positional and thematic uncertainties, addressing research questions 2 and 3 (Section 3.4). Section 4 presents the study results for both the brief comparison and the broader uncertainty analysis (Sections 4.1 and 4.2, respectively). Section 5 discusses results of the brief comparison and the broader uncertainty analysis (Sections 5.1 and 5.2 respectively), followed by a discussion of some more general limitations (Section 5.3) and potential directions for further research (Section 5.4). Finally, the conclusions of the thesis are presented in Section 6. 


\section{Background}

\subsection{Biodiversity strategies in Canada and Ontario}

With the objective of conserving biodiversity, the sustainable use of biological resources, and the fair and equitable sharing of the benefits associated with genetic resources, the United Nations held the Convention on Biological Diversity, in 1992 (United Nations, 1992; Minister of Supply Services Canada, 1995). Later in 1992, Canada became the first industrialized nation to ratify the Convention (Minister of Supply Services Canada, 1995). By 1995, as part of Canada's obligations under the Convention, Canada's Biodiversity Strategy was released (Minister of Supply Services Canada, 1995). Composed by a working group of Federal, Provincial, and Territorial government representatives, it issued a number of strategic directives to governments at these levels, with goals related to those of the Convention. By 2005, the Ontario Biodiversity Council was formed to oversee the implementation of Ontario's Biodiversity Strategy, guided by Canada's Biodiversity Strategy (Ontario Biodiversity Council, 2005, 2011). The most recent version of Ontario's Biodiversity Strategy identifies habitat loss as the primary threat to biodiversity in Ontario, to which agriculture is a significant contributor (Ontario Biodiversity Council, 2011).

\subsection{Biodiversity and agricultural landscapes}

With concern over declining biodiversity in agricultural landscapes associated with habitat loss and intensive, high-yield agriculture, as well as an increasing global

demand for food, questions are being raised about how to better-preserve biodiversity in 
these landscapes without sacrificing food production (Fahrig et al. 2011; Phalan, Balmford, Green, \& Scharlemann, 2011; Gabriel, Sait, Kunin, \& Benton, 2013). Phalan et al. (2011) reviewed suggested solutions, breaking them into two main categories: (1) land-sparing approaches, involving the intensification of practices in currently low-yield farmland, thereby preventing the loss of natural area to new farmland, and (2) wildlifefriendly farming approaches, involving the expansion of farming practices that promote biodiversity within the farmland but generally produce lower yields, meaning that some natural areas would have to be put into production to keep up with food demand. They recognized the potential for mixes of these two approaches, but noted a 'surprisingly scarce' amount of data with which to inform decision-makers.

Ecological study of agricultural landscapes has traditionally been based on a black-and-white-like approach, called patch-matrix representation, where the landscape was looked at in terms of habitat (patch) versus non-habitat (matrix), often semi-natural versus other area (Franklin \& Lindenmayer, 2009; Fahrig et al. 2011; Vasseur et al. 2013). Under this approach, more focus may have been placed on semi-natural area versus cropland, and less on the differences amongst agricultural practices within the cropland itself. However, there have been recent calls for alternative ways of looking at the landscape, such as viewing it in terms of how various elements function for particular species, including those elements that may have traditionally been considered as matrix (Franklin \& Lindenmayer, 2009; Prevedello \& Vieira, 2010; Fahrig et al. 2011; Vasseur et al. 2013).

Fahrig et al. (2011) proposed looking at landscapes in terms of the ecological function provided by various land covers, regardless of whether they provided all of the 
resources required by a species (patch), some of the resources (matrix), or none of the resources (also matrix). They also proposed studying landscape heterogeneity in terms of compositional heterogeneity, the proportions of different land covers, and configurational heterogeneity, the spatial arrangement of the land cover. More specifically, they advocated studying the nature of biodiversity relative to: (1) heterogeneity amongst the more-natural cover types, (2) compositional heterogeneity in the agricultural production area, and (3) configurational heterogeneity in the agricultural production area. Recognizing that the conversion of land from agricultural production to more-natural land cover would be difficult from a policy perspective, they argued that a better understanding of how biodiversity is influenced by heterogeneity within the agricultural production portion of the landscape (potentially easier to control than in the more-natural portion) could help to inform policy makers and contribute to the ecological knowledge base. The framework outlined by Fahrig et al. (2011) guided the Farmland Biodiversity Project (Section 2.6), which this thesis builds upon.

\subsection{Characterizing landscapes with remotely sensed imagery}

Remote sensing, since becoming a commonly used tool in ecology, has been used to create thematic maps of discrete classes from which metrics can be calculated, as well as to estimate continuous ecological variables directly from the imagery (Friedl et al. 2001; Duro et al. 2014). In landscape ecology, metrics of landscape composition and configuration have been commonly derived from thematic land cover maps, including (but not limited to) those produced through image classification (Jensen, 2000; Shao \& Wu, 2008; Fahrig et al. 2011; Uuemaa, Mander, \& Marja, 2013). These maps can exist, 
digitally, in raster format or in vector format, following some form of boundary delineation.

\subsubsection{Image classification}

Image classification, often performed on multispectral RS imagery (data collected in multiple wavebands across the electromagnetic spectrum), refers to the assignment of objects (often pixels) to categories, or classes, based on spectral, spatial, or temporal pattern recognition (Lillesand et al. 2004; Tso \& Mather, 2009). Different approaches to image classification are outlined in this section. Not all of these approaches are used in this study, but many have been used for crop classification within the same study area (van den Berg, 2012; AAFC, n.d.), and are included to provide a better understanding of why the approaches used in this study were selected.

Classification approaches generally fall into one of two categories, either unsupervised classification or supervised classification, though there are approaches that combine both (Lillesand et al. 2004). With unsupervised classification, objects are assigned to data classes based on similar characteristics (often spectral or textural characteristics) which can then be aggregated and labeled as being representative of an information class (i.e. a class that conveys the intended information to the map user) (Tso \& Mather, 2009). With supervised classification, samples with characteristics that are representative of information classes are collected, often called training data, and objects are assigned to classes based on their similarities to these training data characteristics (Tso \& Mather, 2009). The algorithms that perform this pattern recognition and assign objects to classes are often called classifiers. 


\subsubsection{Maximum likelihood and Bayesian classifiers}

The maximum likelihood classifier has traditionally been one of the most commonly used for image classification (Foody, Campbell, Trodd, \& Wood, 1992; Brown, Foody, \& Atkinson, 2009). This parametric classifier derives a posteriori (posterior) probabilities (likelihoods; sometimes called class membership probabilities) of an object belonging to any of the considered classes, and assigns it to the class for which this probability is the greatest (Foody et al. 1992). This first involves defining the classes as multivariate normal (Gaussian) distributions, sometimes called clusters, as determined by the means and variance-covariance matrices of the training data (Foody et al. 1992; Mitchell et al. 2008; Brown et al. 2009). The posterior probability of an object belonging to a given class is then calculated based on the characteristics of the object, relative to these clusters (Foody et al. 1992). A priori (prior) probabilities can also be specified, which can be used to influence (weigh) the calculation of posterior probabilities in accordance with prior knowledge as to the composition of the area being classified (Foody et al. 1992; Lillesand et al. 2004). Lillesand et al. (2004) identify an extension of the maximum likelihood classifier, called the Bayesian classifier, where there is a cost of misclassification assigned to each class. For example, if an object has posterior probabilities of $51 \%$ and $49 \%$ for Classes A and B respectively, but Class A has

a higher cost of misclassification, then the object may be classified as Class B, despite the slightly lower probability.

Brown et al. (2009) outline some of the major benefits and limitations of the maximum likelihood classifier. While relatively easy to understand and easy to use, the 
classifier is limited in that it assumes the data are normally distributed (they are often not) and limited in that a high degree of correlation amongst the input image bands (sometimes the case in high dimensional datasets) can reduce classification accuracy. However, there are approaches to reducing the dimensionality of a dataset (sometimes called feature selection), including principal component analysis, canonical component analysis, or the use of classification and regression trees (Lillesand et al. 2004; Questier, Put, Coomans, Walczak, \& Heyden, 2005).

\subsubsection{Classification trees and Random Forests}

Classification trees, a form of decision tree for categorical data, are another approach to image classification. As described in Questier et al. (2005), they were introduced by Breiman et al. (1984), and involve growing a tree-structured set of decision rules based on repeated binary splits of a sample (a portion of the training set, which form the initial node of the tree), until all end nodes are homogenous in terms of class. All of the predictor variables (image bands) are considered for each split, but only the one that will produce the largest reduction in node impurity (measured by the Gini node impurity index) between the parent and child nodes is selected. Since classification trees tend to over-fit the data, which can lead to reduced accuracy, the trees are usually pruned. This involves specifying a threshold tree size, beyond which any splits are removed, as they only account for a small reduction in error (in the training set) and may just be accounting for noise in the sample. Once built and pruned, class predictions can be made for a case (e.g. a pixel) by filtering it through all decision rules within the tree, with class assignment based on the final (terminal) node that the case ends in. 
The Random Forest is a classifier that is based on classification trees. As described by Cutler, Cutler, \& Stevens (2012), this approach was introduced by Breiman (2001), and involves multiple classification trees being built (when used for classification) on bootstrap samples of the initial training data. For each bootstrap sample (based on resampling of the initial training data, with replacement), a portion (Breiman, 2001, suggested two-thirds) is used to build a classification tree, but with only a random subset of variables considered for each split, and without pruning. The portion that is not used for building the tree, called the out-of-bag data, are reserved for error assessment and estimates of variable importance. With all trees grown, classification can be performed by filtering a case through all trees. The class assignment from each tree acts as a vote, with the case being assigned a class based on the highest number of votes (unweighted), across all trees. Probabilistic estimates of class membership can also be assigned to a case, based on the proportion of votes across all trees (Breiman, Cutler, Liaw, \& Wiener, 2012).

Classification trees have several potential benefits over other classifiers, including that they are non-parametric, they can be less restrictive in terms of the nature of the predictor variables, they are relatively easy to understand and use, they can be more robust to outliers or noise in the variables (when pruned), and they can better-handle datasets with a high dimensionality (Questier et al. 2005; Qi, 2012). In addition to the benefits of classification trees, Random Forests do not suffer from the same degree of over-fitting issues, and only require two or three parameters to be set, which do not require much tuning (Cutler et al. 2012). Despite their relative ease of use, Random Forests have also been shown to produce classification accuracies rivalling those of other 
popular classifiers, such as support vector machines (Pal, 2005; Duro, Franklin, Dubé, 2012).

Due to their ability to rank variables according to measurements of their importance, Random Forests have also seen use in feature selection (Menze et al. 2009; Qi, 2012). These importance measures include a permutation-based measure and a measure based on the Gini importance. The permutation-based importance measure is determined by: (1) recording the error associated with classifying the out-of-bag data, (2) permuting the values in the out-of-bag for individual predictor variables, (3) reclassifying the permuted data, again recording the error, and (4) comparing the increase in error associated with the permuted data relative to that of the original out-of-bag data (Cutler $e t$ al. 2012; Qi, 2012). The Gini-based importance measure is based on summing the reduction in Gini node impurity each time a node is split by a particular predictor variable in a given tree, and summing or averaging that reduction across all trees (Qi, 2012). While the permutation-based importance measure is used more commonly in literature related to Random Forests (Qi, 2012), an issue can arise when using correlated predictors, in which case several of the correlated predictors are assigned a high importance if any one of them is actually important (Cutler et al. 2012). The Gini-based importance measure, on the other hand, can be biased in favor of categorical predictor variables (Qi, 2012).

\subsubsection{Hard and soft classifications}

Traditional image classification involves assigning a single class to each pixel. Under this approach, often called hard or crisp classification, a given pixel is represented 
as belonging completely to its specified class, with no local indication as to the confidence with which the class has been assigned (Foody, 1996, 2002; Canters, 1997). However, there are alternate classification approaches, called soft classifications. Soft classifications include both fuzzy classifications, which operate on the idea that a pixel can have varying degrees of membership in many classes, and softened representations of hard classifications, wherein the degree of confidence associated with hard class assignment can be expressed (Foody et al. 1992; Foody 1996, 2002; Canters, 1997; Eastman \& Laney, 2002; Tso \& Mather, 2009).

Due to the nature of hard classification, the representation of sub-pixel information (information about land cover within the extent of an individual pixel) can be difficult. This can be problematic in the case of mixed pixels, where the area represented by an individual pixel consists of more than one class (Canters, 1997; Eastman \& Laney, 2002). The use of a fuzzy classifier is one way to approach this problem (Eastman \& Laney, 2002; van den Berg, 2012). These classifiers, which can be unsupervised (e.g. fuzzy c-means classifier) or supervised (e.g. fuzzy maximum likelihood classifier), can produce measures that are representative of the proportions of classes within a given pixel (highly correlated, not a direct representation) through non-discrete, fuzzy clustering and fuzzy classification parameters (derived from fuzzy training data, in the supervised case) (Wang, 1990; Foody, 1996; Canters, 1997; Eastman \& Laney, 2002). They can also produce better overall classification accuracy (Wang, 1990).

As an alternative to fuzzy classifiers, some traditionally hard classifiers produce output that can be used as a soft representation, including posterior probabilities from the maximum likelihood classifier or activation levels from neural network classifiers (Foody 
et al. 1992; Foody, 1996). These may not be an accurate representation of class proportions within pixels, but they do provide information as to the confidence with which hard classes can be assigned (Foody et al. 1992; Eastman \& Laney, 2002).

\subsubsection{Manual digitizing and per-field classification}

RS products are not always kept in raster format. Often, areas within an image that can be reasonably assumed to be thematically homogenous are manually delineated, or digitized, based on human interpretation (Wicks, Smith, \& Curran, 2002; Awange \& Kyalo Kiema, 2013). With these areas delineated, often as polygons, thematic maps can be created based on ground referencing, image interpretation, or image analysis. Wicks et al. (2002), argued that imagery characteristics within the extent of individual polygons, or shrunken polygons (to avoid mixed pixels), could be statistically combined to both quantify and minimize the variation within these assumed-homogenous areas. A similar approach can also be used to integrate the results of per-pixel image classifications with manually digitized polygons. Duro et al. (2014) applied a majority filter to assign the results of a per-pixel classification of agricultural crops to manually digitized agricultural field polygons (from the same GIS dataset used in this thesis), thereby producing maps that appeared more realistic, in the sense that fields contained a single crop type.

These approaches are sometimes called per-field, or field-based, classification (Aplin \& Atkinson, 2004; De Wit \& Clevers, 2004; Blaes, Vanhalle, \& Defourny, 2005), and are not necessarily restricted to manual digitizing or polygon-based representation. In fact, there are a variety of approaches to per-field classification (De Wit \& Clevers, 
2004). More recently, there has been a move towards more-automated approaches, as described in Section 2.3.3.

\subsubsection{Object-based image analysis}

There is an increasingly used alternative to per-pixel classification methods, called object-based image analysis (OBIA) (Blaschke, 2010). This technique generally involves using automated image segmentation algorithms to group adjacent pixels into objects that potentially hold some degree of meaning to the analyst (Blaschke, 2010; Blaschke et al. 2014). The segmentation algorithms group pixels based on some measure of similarity (e.g. similarity in spectral values), but can also consider factors such as shape and scale of the similarities (Blaschke et al. 2014). Once objects are defined, statistics characterizing the nature of the pixels within the objects (e.g. mean, variance, maximum, minimum) can provide contextual information beyond that which is available in per-pixel approaches (Blaschke, 2010). This contextual information, which can be further supported by decision rules and multi-scale segmentations, can then be taken into account during image classification to produce a map that may resemble real-world features more closely than per-pixel classifications; though, improvements to overall accuracy may be on a case-by-case basis (Dingle Roberson \& King, 2011; Duro et al. 2012; Blaschke et al. 2014).

\section{$\underline{2.4}$ Error, accuracy, and uncertainty in spatial data}

While definitions can vary, Atkinson \& Foody (2002) describe the differences between error and accuracy, in a remote sensing and GIS context. They define error as 
the difference between a true value and a prediction of that value, and accuracy as a measure of both the lack of bias (associated with systematic error) and the precision (associated with random error). They generally describe uncertainty as associated with the 'unknown' but highlight that, in order to have an understanding of uncertainty, there needs to be an understanding of what is imperfectly known. They further describe uncertainty in two components: (1) ambiguity, the uncertainty associated with hard data, and (2) vagueness, the uncertainty associated with fuzzy data. Zhang \& Goodchild (2002) highlight that error and uncertainty are inherent in data, particularly with the level of abstraction associated with some forms spatial data. They argue that, since truth can be difficult or impossible to obtain, "the notion of uncertainty can be a more realistic view of geographical measurement than the notion of errors" (Zhang \& Goodchild, 2002, p.6).

There are a variety of error and uncertainty sources in spatial data, including attribute error and uncertainty, and location or positional error and uncertainty (Stine \& Hunsaker, 2001; Zhang \& Goodchild, 2002). Attribute errors and uncertainties include those of categorical variables, such as thematic classes assigned by hard image classification. Positional errors and uncertainties include those of feature positions in vector data, and can be present in points, lines, and polygons.

\subsubsection{Error and uncertainty in thematic maps}

The error or accuracy of thematic maps derived through image classification is typically measured by the comparison, at specific locations throughout the map, of hard map classes to classes obtained through a more reliable means, such as ground 
referencing (Foody, 2002). The results of this comparison can be displayed in the form of contingency table, often called the error matrix, or confusion matrix. From this error matrix, several accuracy measures can be calculated, including: (1) the producer's accuracy, a measure of omission error in the map, or the proportion of the reference data, for a given reference class, that were correctly classified, (2) the user's accuracy, a measure of commission error in the map, or the proportion of the correctly classified map units (i.e. pixels, or other fixed areal units) for a given map class, (3) the overall accuracy, the proportion of correctly classified maps units, to incorrectly classified map units, and (4) the Cohen's kappa coefficient, a measure of the difference between the observed accuracy of the map and the accuracy that would be expected due to chance agreement (Foody, 2002; Lillesand et al. 2004).

While user's accuracies from the error matrix may provide global estimates of the confidence in hard class assignment, they tell us little about classification confidence at the pixel level. However, per-pixel estimates of the certainty or ambiguity with which a classifier has assigned pixels to a given class (like those mentioned in Section 2.3.1.3) can often be calculated based on by-products of the hard classification (Foody et al. 1992; Foody, 1996, 2002; Canters, 1997; Mitchell et al. 2008; Brown et al. 2009). For example, Mitchell et al. (2008) used standardized Euclidean distances between pixel values and spectral cluster means to map a per-pixel measure of classification confidence, based on the ratio of this distance for the closest class to the distance for the secondclosest class. Gonçalves, Fonte, Júlio, \& Caetano (2012) compared a normalized entropy measure $\left(\boldsymbol{E}_{\mathbf{n}}\right)$ of per-pixel posterior probabilities of class membership with other measures of uncertainty based on soft classifications. $E_{\mathrm{n}}$ was defined as: 


$$
E_{\mathrm{n}}(x)=\frac{-\sum_{i=1}^{n} p_{i}(x) \log _{2} p_{i}(x)}{\log _{2} n}
$$

where $p_{i}(x)$ is the posterior probability of pixel $x$ belonging to class $i$, and $n$ is the total number of classes (Gonçalves et al. 2012). Entropy-based measures have long been attractive for quantifying classification ambiguity (Foody, 1996).

\subsubsection{Error and uncertainty in point and linear features}

Positional error and uncertainty in point and line data can be measured based on repeated measures of the position, ideally from different interpreters (Fortin \& Edwards, 2001).

For points features (e.g. vertices), distances between repeated positional measurements (test sources) and a reference position can be used to estimate error and uncertainty (Shi, Ehlers, \& Tempfli, 1999; Zhang \& Goodchild, 2002). A positional measurement with a higher accuracy should be used as the reference (Goodchild \& Hunter, 1997; Zhang \& Goodchild, 2002). Error can be represented by the root mean square error (RMSE), whereas uncertainty can be represented with probability density functions (PDFs) in orthogonal, horizontal directions (i.e. the $\mathrm{x}$ and $\mathrm{y}$ directions), centered on the map point, with variances based on the distance measures (Shi et al. 1999; Zhang \& Goodchild, 2002). Normal PDFs are often used (Shi et al.1999; Shi \& Liu, 2000; Zhang \& Goodchild, 2002; Tong, Sun, Fan, Goodchild, \& Shi, 2013).

For line features, positional error and uncertainty has traditionally been represented by the epsilon error band model, a buffer of a global width (epsilon) around a digitized line segment, which is assumed to contain the true line position, but is sensitive 
to outliers (Goodchild \& Hunter, 1997; Fortin \& Edwards, 2001; Zhang \& Goodchild, 2002). Goodchild \& Hunter (1997) proposed an alternate distance measure, based on the buffer distance around an assumed-true line that contains a certain percentile of the length of a test line. Shi (1998) and Shi et al. (1999) represented positional uncertainty in line segments with probabilistic representations, defined by PDFs of positional uncertainty in the lines' endpoints, between which errors were assumed to be independent. Later, Shi \& Liu (2000) proposed the G-band error band model, which involves modelling the PDF at any point along a line segment based on the endpoint PDFs and the degree of correlation between them.

\subsection{Uncertainty analysis and Monte Carlo methods}

Uncertainty analysis, sometimes referred to as error propagation, is an important process for assessing whether the quality of model output meets the requirements of decision-makers (Crosetto \& Tarantola, 2001). It involves identifying and modelling all sources of error and uncertainty in a model's inputs and parameters, and determining the degree to which model outputs are influenced by their propagation (Crosetto et al. 2000; Crosetto \& Tarantola, 2001; Crosetto et al. 2001). It can be accompanied with sensitivity analysis, wherein the quantified output uncertainty is apportioned to individual input sources (Crosetto \& Tarantola, 2001). Uncertainty analysis and sensitivity analysis can be performed analytically in some cases, though, as mentioned earlier, MC simulation can be an effective alternative, due to its ability to treat the model as a black box, not requiring assumptions about the model's structure (Crosetto \& Tarantola, 2001; Crosetto et al. 2001). 


\subsubsection{Monte Carlo simulation}

MC simulation is one of the most widely used approaches for uncertainty analysis, including the quality assessment of GIS-based products (Canters, 1997; Crosetto \& Tarantola, 2001; Brown \& Heuvelink, 2007). In the uncertainty analysis case, MC simulation involves four steps, as described by Crosetto et al. (2000), Crosetto \& Tarantola (2001), and Crosetto et al. (2001):

(1) assign PDF representations to model input factors (e.g. uncertainty in an individual vertex's position, or uncertainty in assigning a class to an individual field);

(2) by repeatedly sampling the PDFs, generate multiple error sensitized input factor samples, called realizations, which can be used to generate realizations of model inputs (e.g. landscape metrics) and parameters;

(3) repeatedly apply the model with the realizations of inputs and parameters, generating a distribution of output values; and

(4) analyze the output distribution for the characteristics of interest.

Sampling of the PDFs can be performed via inverse-transform sampling for continuous PDFs, or discrete inverse-transform sampling for discrete PDFs (Kroese, Taimre, \& Botev, 2011). Both of these involve representing the PDF as a cumulative density function (CDF) from which samples are drawn using uniform pseudo-random numbers (PRNs) between 0 and 1 (Kroese et al. 2011). An illustrated example of these sampling approaches is provided in Figure 2.1, below. 

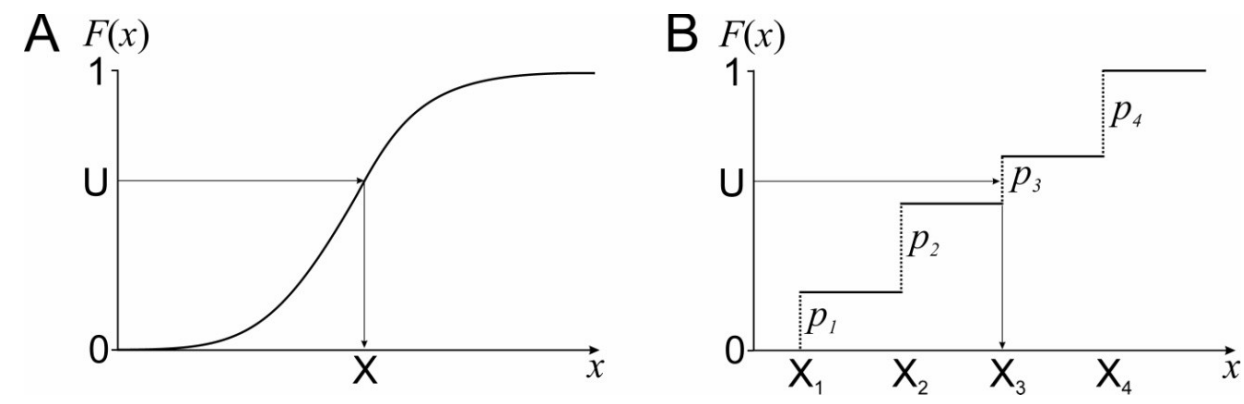

Figure 2.1 Illustrated examples of (A) inverse transform sampling, and (B) discrete inverse transform sampling. $\mathrm{U}$, a uniform random number between 0 and 1 , is used to sample a continuous or discrete $\mathrm{CDF}, F$ (discrete probabilities represent by $p_{1}$ through $p_{4}$ ). $\mathrm{X}$ is a possible value of variable $x$. This figure is based on figures 3.1 and 3.2 of Kroese et al. 2011, pp.46-46.

There is no standard number of realizations that needs to be generated, and the number required can vary depending on the application. Since generating more realizations and performing more model runs will allow the output distribution to be better-defined, the number required depends on the desired level of precision with which the characteristics of interest can be estimated (Mowrer, 1997; Crosetto \& Tarantola, 2001). Mowrer (1997) plotted the cumulative variance of the variables of interest against the number of realizations, to get a visual sense of how many realizations were required to stabilize the estimates.

Estimates from MC simulation that have been determined with a high level of precision can still be inaccurate if the approaches used to generate realizations are inappropriate (Crosetto et al. 2001). There are several MC methods to generating realizations, each of which may be best suited to particular systems or processes (Kroese 
et al. 2011). For example, when generating realizations of time-series data, where the outcome of the next time point is dependent on the outcome, or state, of the current time point, conditioning of the PDF for the next time point as a function of the current state, may be required (Gilks, Richardson, \& Spiegelhalter, 1996; Kroese et al. 2011). In this case, Markov Chain Monte Carlo methods may be useful. Similarly, when generating a realization of a categorical raster map, wherein there is some degree of spatial autocorrelation between pixels, conditioning of the PDF (i.e. posterior probabilities of class membership) for the next pixel, based on the current spatial distribution of pixel realizations, may be required. Sequential Indicator Simulation (SIS) may be useful in such a case.

\subsubsection{Sequential indicator simulation}

SIS is a commonly used geostatistical MC method for generating realizations of categorical maps, while considering spatial autocorrelation (de Bruin, 2000; Kyriakidis \& Dungan, 2001; Zhang \& Goodchild, 2002; Magnussen \& de Bruin, 2003). It requires that the degree of spatial autocorrelation between classes (categories) has been characterized (e.g. with semi-variograms). This information, in addition to location-specific probabilities of class membership and an initial set of high-accuracy reference data (distributed throughout the map), is considered when generating map realizations (de Bruin, 2000).

As described by de Bruin (2000) and Kyriakidis \& Dungan (2001), the SIS approach involves establishing a random path that visits image pixels, one at a time. While visiting pixels along this path, kriging is used to update the PDF (and CDF) of the current 
pixel, based on characteristics of a conditioning set (a group of surrounding pixels) and information regarding the degree of spatial autocorrelation. Once the PDF (and CDF) of the pixel has been conditioned, it is sampled (e.g. using the discrete inverse transform approach), and the pixel is assigned a simulated value. The conditioning set, initially made up of only high-accuracy reference data, is then updated with the simulated value (e.g. a hard thematic class) for the current pixel. Pixels along the random path are visited until all pixels have been assigned a simulated values, constituting a full map realization.

\subsubsection{Field-based Monte Carlo simulation}

Canters (1997) proposed a relatively simple simulation approach that was shown to better preserve the spatial structure of map realizations (in the presence of spatial autocorrelation) than simulating pixels independently. Called field-based simulation, it involves segmenting the image into fields (not necessarily agricultural fields) and drawing a common PRN for all pixels in the same field, when sampling from their CDFs. The approach does not involve conditioning PDFs and, therefore, does not require a set of reference data distributed throughout the landscape to act as an initial conditioning set. Pixels within a field that share a similar PDF are more likely to vary together within a single map realization (guaranteed to vary together if PDFs are identical) than they would under independent simulation.

The segmentation approaches investigated by Canters (1997) were based on grouping contiguous pixels with similar probabilities of class membership, but the paper recognized digitized field boundaries as an alternative. The study found that, while independent pixel-based and field-based simulation approaches produced similar 
estimates for the mean of the output distribution, the variance of the output distribution had potential to be much larger under the field-based approach (largest with the highest degree of grouping). The field-based approach also resulted in better within-field structure preservation within realizations. The field-based simulation approach was again used by Canters, De Genst, \& Dufourmont (2002) while studying the effects of uncertainty in land cover classification and digital elevation model error on structural landscape classification, but did not involve a comparison of simulation approaches.

\section{$\underline{2.6 \quad \text { Farmland Biodiversity Project }}$}

A large, multi-disciplinary project, called the Farmland Biodiversity Project, is being conducted at Carleton University's Geomatics and Landscape Ecology Laboratory. It seeks to develop a better understanding of how agricultural landscapes can be structured, in terms of composition or configuration, so that biodiversity can be enhanced without removing land from agricultural production (Fahrig et al. 2011). It is directed towards informing policy regarding the management of these landscapes, in addition to contributing to the ecological knowledge base (Fahrig et al. 2011).

The project involved building several statistical models, each predicting one of several forms of biodiversity (or abundance) for one of several taxonomic groups, selected based on potential differences in their response to landscape pattern, the ecosystem services that they provide, and the ease with which they can be sampled (Fahrig et al. 2011; Fahrig et al. 2015). Counts of individuals within each taxon were performed at four points or transects (depending on the taxon) within several $1 \mathrm{~km}$ by 1 $\mathrm{km}$ agricultural landscapes across eastern Ontario (Duro et al. 2014). The data were 
collected during the 2011 and 2012 growing seasons. From these counts, alpha, beta, and gamma diversity, as well as abundance, were calculated for each landscape. Duro et al. (2014), citing Lande (1996), Wagner et al. (2000), Whittaker et al. (2001), and Crist et al. (2003), describe the calculation of mean alpha diversity as the mean number of species across the four sampling locations, the calculation of gamma diversity as the total number of species across the four sampling locations, and the calculation of beta diversity as the difference between the gamma diversity and the mean alpha diversity of the landscape. With this approach, gamma diversity, the total diversity of a landscape, is partitioned into alpha and beta diversity, measures of local diversity at sampling points within the landscape and turnover between species across the sampling points respectively (Crist et al. 2003; Duro et al. 2014). This additive partitioning between measurements at different scales (e.g. landscape scale versus sampled habitats within the landscape) allows for the hierarchical analysis of diversity patterns, as opposed to analysis at a fixed scale (Crist et al. 2003). Finally, abundance was the total number of observed individuals of a given taxon, across the four sampling locations (Fahrig et al. 2015).

Each of the landscapes was mapped out to $3 \mathrm{~km}$ by $3 \mathrm{~km}$ in extent, based on air photo digitizing and ground referencing of crop types (further details available in Section 3.2.1). From the innermost $1 \mathrm{~km}$ by $1 \mathrm{~km}$ region of these maps, three sets of landscape metrics were calculated, including the proportion of land in agricultural production (P.AG), the mean agricultural field size (MFS), and the Shannon Diversity Index of the crop types that were present in the landscape (SHDI). The crop types considered in this analysis were corn, soybean, hay, pasture, legumes, cereals, fallow, apple orchard, 
canola, mixed vegetables, strawberries (considered as mixed vegetable for SHDI calculation), peas, sod, and sunflower (definitions of land cover classes provided in Section 3.2.1). The Shannon Diversity Index, as described by Hurlbert (1971), is:

$$
\text { SHDI }=-\sum_{i=1}^{n} \frac{N_{i}}{N} \log \frac{N_{i}}{N}
$$

where $N_{i}$ is the total area of crop class $i$ (of $n$ classes) and $N$ is total area of all crop classes (symbology described in the context of this project). SHDI was the compositional landscape characteristic of interest to the project, while MFS was the configurational characteristic of interest (Pasher et al. 2013).

The landscapes were selected based on an analysis by Pasher et al. (2013). The study involved mapping crop types throughout eastern Ontario using supervised image classification and image segmentation. SHDI and MFS were calculated across this map at scales ranging from $1 \mathrm{~km}$ by $1 \mathrm{~km}$ to $7.5 \mathrm{~km}$ by $7.5 \mathrm{~km}$. Using these metrics, they identified a limit to the landscape extents $(3 \mathrm{~km}$ by $3 \mathrm{~km})$ at and below which the degree of variation in these metrics met the desired level. They further identified several potential landscapes, within areas of $60 \%$ to $90 \%$ P.AG, that avoided spatial autocorrelation in the metrics between landscapes and avoided correlation between the two metrics across all landscapes.

van den Berg (2012) performed image classifications of areas throughout the study area, comparing an unsupervised fuzzy classification with a hard maximum likelihood classification. The study found that the fuzzy classification provided a better representation of landscape heterogeneity near field boundaries than the hard maximum 
likelihood classification, though the fuzzy classifier did struggle with high degrees of classification error in some regions

\subsubsection{Modelling}

The Farmland Biodiversity Project involves several modelling sub-projects, some of which are ongoing. The models analyzed by this thesis were provided by researchers associated with Fahrig et al. (2015), and are very similar to some of those described in Duro et al. (2014). There were 23 models provided, across 6 taxa (beetles, birds, butterflies, plants, spiders, and syrphids) and 4 biodiversity types (abundance included as a biodiversity type for ease of explanation). There were no abundance data for plants. This section summarizes how those models provided by Fahrig et al. (2015) were derived, as similar forms and methods used in that modelling were used for uncertainty analysis performed in this thesis. However, since this research was performed while the models published in Fahrig et al. (2015) were being developed, the models used in this thesis are different, older versions of those published.

For the models provided, any given biodiversity type of any given taxon (response variable) was linearly regressed on the landscape metrics (i.e. P.AG, MFS, SHDI were predictor variables), along with a year indicator variable (year $=1$ for data from year 2012, and 0 for year 2011) to account for differences between years. However, given that variance in biodiversity might not be 'best' explained by all three predictors, the regression was also performed for all possible pairs of the predictors, and all predictors individually (all including the year indicator). So, for any given response variable, there were seven candidate models. Prior to modelling, the metrics had been 
centered (subtract the mean) and scaled (divide by the standard deviation), so the coefficients of the candidate models were beta coefficients $\left(\beta_{\mathrm{P.AG}}, \beta_{\mathrm{MFS}}\right.$, and $\left.\beta_{\mathrm{SHDI}}\right)$.

While a single model may have been selected as the 'best', based on some criterion (e.g. coefficient of determination, or the adjusted coefficient of determination), this would have meant that the established relationships would be conditional on that model being selected (Anderson, 2008). To establish unconditional, more-robust relationships between biodiversity and the landscape characteristics, a model averaging approach was used (Burnham \& Anderson, 2002; Anderson, 2008; Duro et al. 2014). The approach involves, first, comparing candidate models in terms of their ability to explain the variance in the data, penalized for model complexity (the number of model parameters) via the second-order bias corrected Akaike's Information Criterion (AICc) (Anderson, 2008). Weights are then calculated based on the AICc values of each candidate model and used to take weighted average of all model parameters, across those models in which they appear (Anderson, 2008). The model-averaged parameters obtained by this approach were used as the parameters of the final biodiversity models. Additionally, the approach produced unconditional parameter variance estimates that consider: (1) the uncertainty associated with sample, given the model, and (2) the uncertainty associated with model selection (Burnham \& Anderson, 2002; Anderson, 2008).

The models that were based on this averaging approach, using landscape metrics from the ground referenced landscape maps, will be referred to as the original biodiversity models. The landscape metrics (P.AG, MFS, and SHDI) based on the ground referenced landscape maps will be referred to as the original landscape metrics. 
The development of the models was outside of the scope of this thesis, falling under the larger, overarching Farmland Biodiversity Project. This thesis focuses on the uncertainty in the relationships (model coefficients) established by those models, under the scenario that those models were fit on metrics from an alternate set of landscape maps, based on digitizing and image classification (Section 1.2). Therefore, the relationships presented in this thesis differ from the relationships of the original models. 


\section{Methods}

This section describes the methods used to address the research questions (Section 1.3). Sections 3.1 and 3.2 provide basic information about the study area and the data, most of which were provided through the Farmland Biodiversity Project. Methods related to addressing the research questions of this study are presented in Sections 3.3 and 3.4. Section 3.3 describes methods that were aimed specifically at addressing research question 1. Section 3.4 describes those methods for addressing research questions 2 and 3.

All PRNs generated in these methods are based on the Mersenne-Twister algorithm (Brown \& Heuvelink, 2007; The R Core Team, 2014). The algorithm was proposed by Matsumoto \& Nishimura (1998), stating that they felt it was the most suitable for MC simulation, relative to other common PRN generators of the time. It was designed specifically for generating uniform PRNs between 0 and 1 (at 32-bits), and remains a good performer, with a very large period (many numbers generated before duplicates appear), good speed, and good ability to generate uniform distributions (Matsumoto \& Nishimura, 1998; Kroese et al. 2011).

It should be noted that there is a change in terminology between Sections 3.3 and 3.4 (and their corresponding Results and Discussion sections), due to comparisons within different contexts. In Section 3.3 (and associated Results and Discussion sections), when comparing approaches to preserving within-field structure, there is a simulation approach referred to as homogenous field-based simulation. In terms of assigning simulated thematic classes, this approach is the same as independent field simulation, which is 
discussed in Section 3.4 (and associated sections) where different approaches to preserving landscape structure are explored, all with homogenous fields.

All vector data processing (not including initial data obtained from the Farmland Biodiversity Project), unless otherwise stated, was performed with ArcGIS Desktop 10.1 or 10.2 (ESRI, 2012, 2013a). The image classifications performed in Section 3.3 were performed with IDRISI Taiga (Version 16.1) (Eastman, 2009a). The simulations were performed in 'the R environment for statistical computing' (Version 3.0.1) (The R Core Team, 2013), with the exception of a portion of those performed in Section 3.4.

\section{$\underline{3.1}$ Study area}

This thesis is situated in the same study area as the Farmland Biodiversity Project (Section 2.6). The landscapes were distributed throughout a portion of eastern Ontario, roughly southeast of the City of Ottawa, bounded by $74^{\circ} 47^{\prime} \mathrm{W}$ to $75^{\circ} 57^{\prime} \mathrm{W}$ longitude and $44^{\circ} 52^{\prime} \mathrm{N}$ to $45^{\circ} 29^{\prime} \mathrm{N}$ latitude (Fig. 3.1).

The study area lies in the Mixedwood Plains ecozone, the most biologically diverse ecozone in Canada, characterized by rich soils and a moderate climate (Ontario Biodiversity Council, 2010; Duro et al. 2014). The ecozone's landscape, previously dominated by forests, wetlands, prairies, and alvars, is now dominated by a combination of human settlement and agriculture (Ontario Biodiversity Council, 2010, 2011).

In 2011, the general area (three census divisions containing all but 5 of the 93 landscapes) had just over 359,000 hectares of land in agricultural production (including crops, pasture, and summerfallow) (OMAFRA, n.d.; Statistics Canada, 2011). This 
production area consisted of approximately $31 \%$ corn, $28 \%$ soybean, $24 \%$ hay (including grass and legume mixtures), $10 \%$ pasture, $3 \%$ assorted cereals (wheat, oats, barley and mixed grains), and less than $1 \%$ summerfallow (OMAFRA, n.d.; Statistics Canada, 2011).
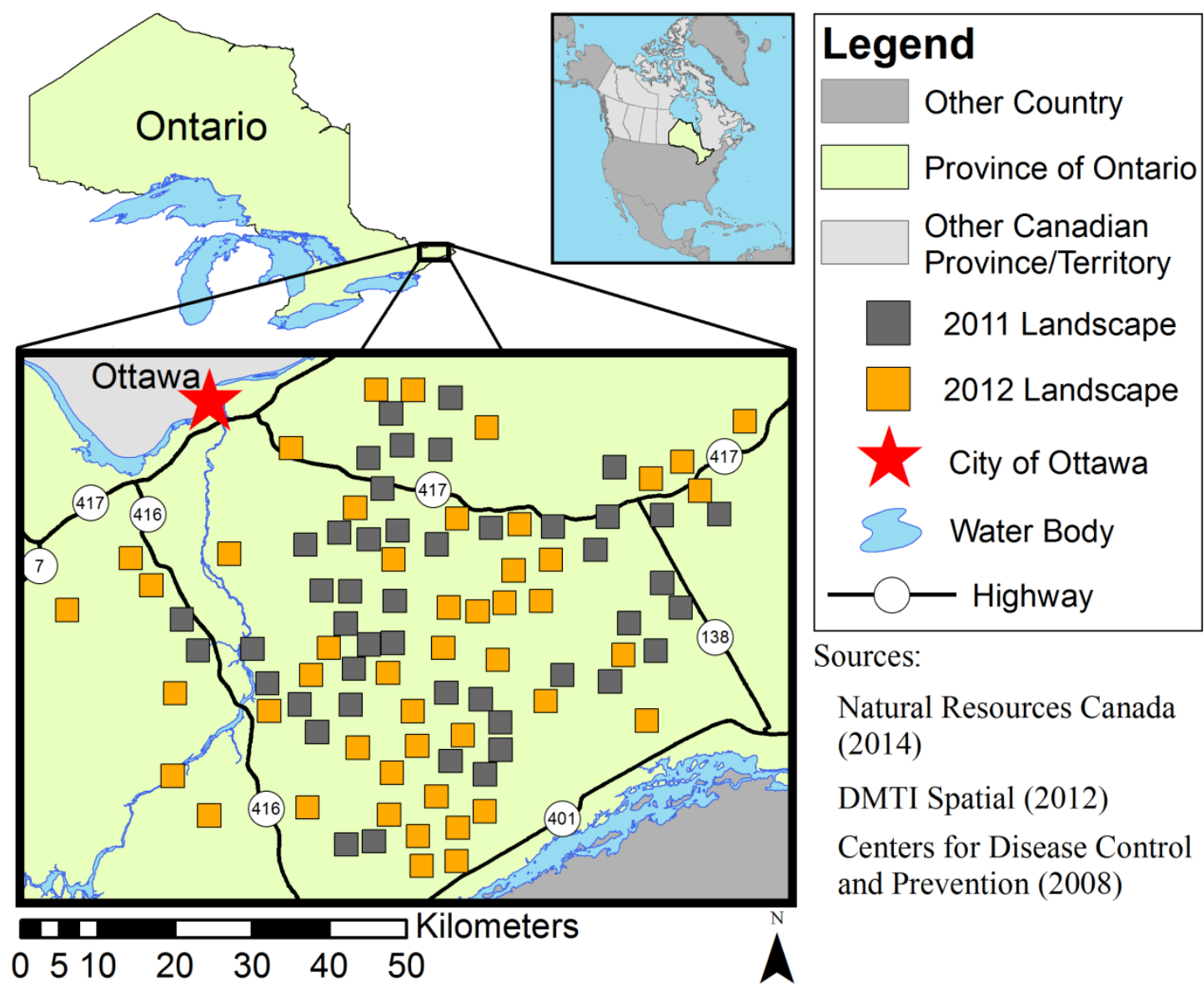

Sources:

Natural Resources Canada (2014)

DMTI Spatial (2012)

Centers for Disease Control and Prevention (2008)

Figure 3.1 Distribution of the study landscapes throughout eastern Ontario. Layout inspired by van den Berg (2012), Figure 2, p. 23 and Dingle Robertson \& King (2011) Figure 1, p. 1506 . Compiled in ArcGIS 10.2 (ESRI, 2013a). 


\subsubsection{Ontario growing season}

The 2011 Ontario growing season, as described in the Ontario Ministry of Agriculture, Food and Rural Affairs' (OMAFRA) (2011a, 2011b, 2011c, 2011d, 2011e) Field Crop Reports, started with a cool and wet April and early May, resulting in delayed planting or seeding of some crops (e.g. corn, soybean, and spring cereals), spring kill of some winter cereals, delayed seeding of forage crops (e.g. hay and legumes) and pastures, and stunted grass growth for forage crops and pastures. In many cases, corn and soybean planting was delayed until late May or early June. Later seeding of the forage crops resulted in more variability among these crops. The conditions also led to some legumes (e.g. alfalfa) remaining dormant for longer than normal, resulting in increased proportions of grass in grass-legume mixes.

The reports (OMAFRA, 2011a, 2011b, 2011c, 2011d, 2011e) state that a dry July stressed many corn and soybean crops, but was followed by excellent weather conditions in August and September, aiding in their recovery and resulting in better than average yields. Final yields for cereals were quite variable. With the dryer July conditions, the quality of mid-summer cuts of forage crops was variable and pasture growth was slowed. However, the quality of forage crop cuts and pasture growth increased with better conditions towards the end of the summer. Conditions remained excellent for pasture growth in September and October.

\subsubsection{Ontario growing season}

The 2012 Ontario growing season had a warm and dry start, according to OMAFRA's (2012a, 2012b, 2012c, 2012d, 2012e) Field Crop Reports. A dry March and 
April resulted in early planting/seeding of corn, soybean, spring wheat, and forage crops, as well as early pasture growth. Some rainfall was received in May and June, but July brought very high temperatures and very low precipitation, stressing crops. Growing conditions improved in August and September, allowing some crops, such as corn and soybean, to recover. Overall, good yields were recorded for corn, soybean, and cereals, while low yields were recorded for forage crops.

\subsection{Data}

This section provides details on vector data obtained from the Farmland Biodiversity Project, some supplementary data from Agriculture and Agri-Food Canada (AAFC), the air photos used, and satellite imagery used.

\subsubsection{Data from the Farmland Biodiversity Project}

Data from the Farmland Biodiversity Project (Section 2.6) included:

- 93 ground referenced landscape maps (46 from year-2011 and 47 from year2012),

- biodiversity measurements for each of the study landscapes,

- landscape metrics for each of the study landscapes, and

- high resolution air photos (Section 3.2.3) and satellite imagery (Section 3.2.4).

The 93 ground referenced landscape maps were stored as a GIS vector dataset, with polygon features. Each map represented a $3 \mathrm{~km}$ by $3 \mathrm{~km}$ landscape and was 
accompanied by a copy representing just the innermost $1 \mathrm{~km}$ by $1 \mathrm{~km}$ center of each landscape. For ease of distinguishing between these extents, the $3 \mathrm{~km}$ by $3 \mathrm{~km}$ extents will be referred to as landscapes, while the inner $1 \mathrm{~km}$ by $1 \mathrm{~km}$ will be called landscape centers (LCs). However, it should be noted that the term 'landscape' does not necessarily refer to a single extent.

Map features had been delineated through the digitizing of a year-2008 air photo, covering the study area, and were later updated based on high resolution air photos from their respective growing seasons (years 2011 and 2012). These features represented a variety of land covers including urban areas, forests, riparian/wetland areas, water, abandoned agricultural fields, and active agricultural fields. Features were assigned thematic classes based on air photo interpretation, with the exception of active agricultural fields which were classified by crop type (pasture and fallow considered as crops) through ground referencing. The crop classes are shown in Table 3.1, below. In this thesis, references to these landscape maps will only refer to polygons representing agricultural fields (or abandoned fields in some cases) in these maps. 
Table 3.1 Agricultural field class definitions (Abandoned not considered as agricultural field).

\begin{tabular}{|c|c|}
\hline Class & Description \\
\hline Corn $^{*}$ & A field planted with corn \\
\hline Soybean $^{*}$ & A field planted with soybean \\
\hline $\mathrm{Hay}^{*}$ & A field planted with grass hay or a grass-legume mix of $>75 \%$ grass \\
\hline Legumes ${ }^{*}$ & $\begin{array}{l}\text { A field planted with legume hay or a grass-legume mix of } \geq 25 \% \\
\text { legumes }\end{array}$ \\
\hline Pasture $^{*}$ & $\begin{array}{l}\text { A field where livestock are grazing or evidence of livestock grazing is } \\
\text { seen }\end{array}$ \\
\hline Cereals $^{*}$ & $\begin{array}{l}\text { A field planted with cereals, including wheat, oats, barley, grain mixes, } \\
\text { etc. }\end{array}$ \\
\hline Fallow ${ }^{*}$ & $\begin{array}{l}\text { A field that was not in production during the current growing season, } \\
\text { but not left unmaintained to the point where there is overgrowth by } \\
\text { woody vegetation }\end{array}$ \\
\hline Apple & An apple orchard \\
\hline Canola & A field planted with canola \\
\hline $\begin{array}{l}\text { Mixed } \\
\text { vegetables }\end{array}$ & A field planted with a type of vegetable or a mixture of vegetables \\
\hline Peas & A field planted with peas \\
\hline Sod & A field planted with grass sod \\
\hline Strawberry & A field planted with strawberries \\
\hline Sunflower & A field planted with sunflowers \\
\hline Abandoned $^{* *}$ & $\begin{array}{l}\text { A field that has not been in production for at least two years, showing } \\
\text { overgrowth by woody vegetation }\end{array}$ \\
\hline Unknown & A field of an unknown or unobserved crop type \\
\hline
\end{tabular}

Fields within the LCs had been ground referenced from within the fields, by ground referencing teams. These teams also checked field edges within the LCs. Fields outside of the LCs, but still within the landscapes, were ground referenced by viewing from the nearest public road.

While the data outlined in this section had been collected under the larger Farmland Biodiversity Project, those involved in this thesis study had been involved in 
the larger project. This involvement included participation in digitizing and ground referencing, during the production of the year-2012 landscape maps.

\subsubsection{Data from Agriculture and Agri-Food Canada}

A dataset of ground referenced crop types from AAFC's Annual Crop Inventory was available. The dataset, used for classifier training and reference purposes, consisted of a series of polygons identifying crop types within fields adjacent to public roads. The AAFC project maps a variety of crop types across Canada, with an overall accuracy of at least 85\% (AAFC, n.d., 2013). At this accuracy, it was assumed that the ground referenced dataset was of high quality and useful as a supplement to ground reference data from the Farmland Biodiversity Project.

\subsubsection{Air photos}

A colour air photo from 2008, covering most of eastern Ontario, was available from the Ontario Ministry of Natural Resources, via the Farmland Biodiversity Project. The $50 \mathrm{~cm}$ resolution air photo had a Universal Transverse Mercator (Zone 18) projection, using the North American Datum of 1983, and had a precision of \pm 1 meter (Ontario Ministry of Natural Resources, 2009).

Colour air photos from years 2011 and 2012 were also available from the Farmland Biodiversity Project. These photos fully covered the majority of the study landscapes (one landscape from 2012 did not have full coverage, and a small number were partially obscured by cloud) in their respective growing seasons. Many of the year2011 photos had been georectified to QuickBird satellite imagery of the study area, with 
a RMSE of 1.07 meters (van den Berg, 2012). The year-2012 air photos had been georectified to the year-2008 air photo with an average RMSE of 3.06 meters. The photos had an initial resolution of $40 \mathrm{~cm}$ (Fahrig et al. 2015) though many had a cell size of close to $50 \mathrm{~cm}$ following processing.

\subsubsection{Satellite imagery}

Partial coverage of the study area by multispectral QuickBird (QB) imagery was available from the Farmland Biodiversity Project. 20 of the 46 landscapes from year2011 and 20 of the 47 landscapes from year-2012 had near-full coverage by this imagery. The imagery had a pixel size of 2.4 meters, with spectral characteristics outlined in Table 3.2 .

Table 3.2 QuickBird and Thematic Mapper spectral characteristics (Digital Globe, n.d.; USGS, n.d. b; Lillesand et al. 2004). Band 6 (thermal) of Thematic Mapper imagery is not included.

\begin{tabular}{cccc}
\hline Imagery & $\begin{array}{c}\text { Band } \\
\text { number }\end{array}$ & Spectral region [Colour] & Wavelength $(\mu \mathrm{m})$ \\
\hline QuickBird & 1 & Visible [Blue] & $0.43-0.55$ \\
(QB) & 2 & Visible [Green] & $0.47-0.62$ \\
& 3 & Visible [Red] & $0.59-0.71$ \\
& 4 & Near infrared & $0.72-0.92$ \\
\hline Landsat-5 & 1 & Visible [Blue] & $0.45-0.52$ \\
Thematic & 3 & Visible [Green] & $0.52-0.60$ \\
Mapper & 4 & Visible [Red] & $0.63-0.69$ \\
(TM) & 5 & Near infrared & $0.76-0.90$ \\
& 7 & Mid-infrared & $1.55-1.75$ \\
& & Mid-infrared & $2.08-2.35$ \\
\hline
\end{tabular}


Several Landsat-5 Thematic Mapper (TM) scenes were available for the year2011 growing season, with acquisition dates of 3 June, 19 June, 5 July, and 9 October (NASA Landsat Program, 2011). These images have a pixel size of 30 meters and six spectral bands (excluding Band 6, the thermal band), ranging from the visible to the midinfrared (Table 3.2). The imagery had undergone systematic radiometric and geometric correction (Level 1T processing) and had a Universal Transverse Mercator (Zone 18) projection, using the World Geodetic System of 1984 (USGS, n.d. a).

The TM image from June and October were quite free of cloud and haze (Fig. 3.2 A, B, D, below). The 5 July TM image, however, did have cloud cover over some landscapes, with a light to moderate haze covering much of the study area (Fig 3.2 C).

The Normalized Difference Vegetation Index (NDVI) was calculated for each date as:

$$
\text { NDVI }=\frac{\text { Near infrared }- \text { Red }}{\text { Near infrared }+ \text { Red }}
$$

where the near infrared was represented by Band 4 in the QB or TM imagery, and the red was represented by Band 3 (Jensen, 2000; Davidson \& Csillag, 2001). Davidson \& Csillag (2001) found ground-based NDVI measurements (bands corresponding to TM Bands 3 and 4) to have a logarithmic relationship with above-ground biomass for native prairie vegetation. Of the common vegetation indices compared, the study found NDVI to be amongst the best in terms of explaining the variance in above-ground biomass. 

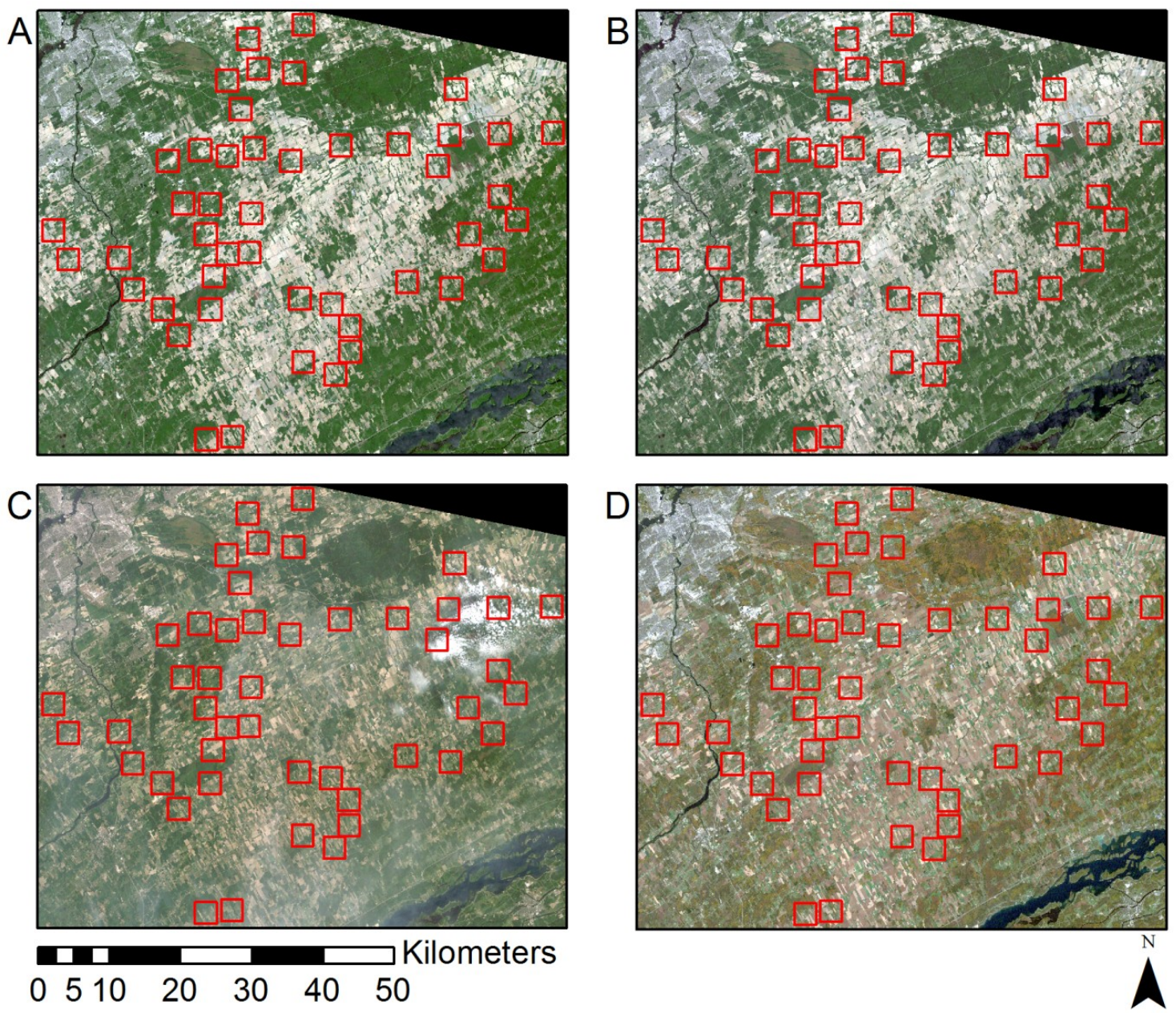

Figure 3.2 Natural colour composite TM images of the study area from (A) 3 June, 2011, (B) 19 June, 2011, (C) 5 July, 2011, and (D) 9 October, 2011. Boundaries of the study landscapes from year-2011 are shown in red.

Landsat-7 Enhanced Thematic Mapper imagery was available for years 2011 and 2012. However, for both years, the imagery had significant gaps due to a combination of cloud cover and the failure of the sensor's Scan Line Corrector (USGS, n.d. c). The nature of these gaps meant that the degree of coverage would be quite variable, even within individual landscapes. For this reason, the Landsat-7 imagery was not used for this thesis. 


\subsection{Simulation of the effect of thematic uncertainty on SHDI}

MC simulation approaches to quantifying uncertainty in thematic maps can differ in terms of their assumptions and how they preserve the spatial structure of the landscape. These differences can lead to significant differences in the characteristics of the output distributions (Canters, 1997; de Bruin, 2000; Crosetto et al. 2001).

Canters (1997) compared output distributions of class area from field-based simulation approaches to the distributions from a spatially independent (independent pixels) approach (Section 2.5.3). The study found that the field-based approaches tended to better-preserve landscape structure within realizations and that, while the means of the output distributions were similar, the output distributions from the field-based approaches were more variable. Both of the approaches compared by Canters (1997) still allowed pixels within an individual field to vary separately (unless they have identical PDFs). However, if the spatial boundaries of agricultural fields have been well-defined (e.g. through digitizing high resolution air photos), it may be safe to assume that the fields are thematically homogenous. Considering these fields as thematically homogenous objects during simulation may also influence the characteristics of the output distributions.

The comparison performed here was aimed at addressing research question 1 (Section 1.3), comparing different simulation approaches to considering within-field spatially correlated thematic error. Canters (1997), using a fuzzy maximum likelihood classifier, compared field-based simulation approaches with independent pixel-based approaches, in terms of their means, variances, and within-field structure preservation (Section 2.5.3). The comparison performed here was aimed at confirming whether the effects of using 
field-based simulation were similar to those found by Canters (1997), given a different landscape metric, a different classification scheme, different imagery, and a different classifier. This comparison was extended to consider the effects of a homogenous fieldbased simulation approach, described later. The landscape metric of interest was SHDI (as measured from the full $3 \mathrm{~km}$ by $3 \mathrm{~km}$ extents). Three different image sets, based on TM and QB imagery were used in the comparison. Also, posterior probabilities from the traditional maximum likelihood classifier, as opposed to the fuzzy maximum likelihood classifier, were used. In addition to confirming the results of Canters (1997), it was hoped that the comparison could identify a classification and simulation approach for which the output distribution was more representative of reference SHDI values.

\subsubsection{Site selection}

Landscapes that were used in the comparison were limited to the extent of one of the QB images. All training and reference data were from within this extent. van den Berg (2012) had used a similar approach for a comparison of fuzzy and maximum likelihood classifications of QB and TM imagery within the same study area.

Site options were narrowed down to QB images 2 and 3 (numbered QB images shown in Fig. 3.3). Images 2 and 3 were acquired in August, when the crops appeared to be in better health and had fuller canopies compared to the July acquisitions of images 1, 4, and 5 (Fig. 3.4). QB image 4 was not considered, as it only contained one full study landscape (Fig. 3.3). 


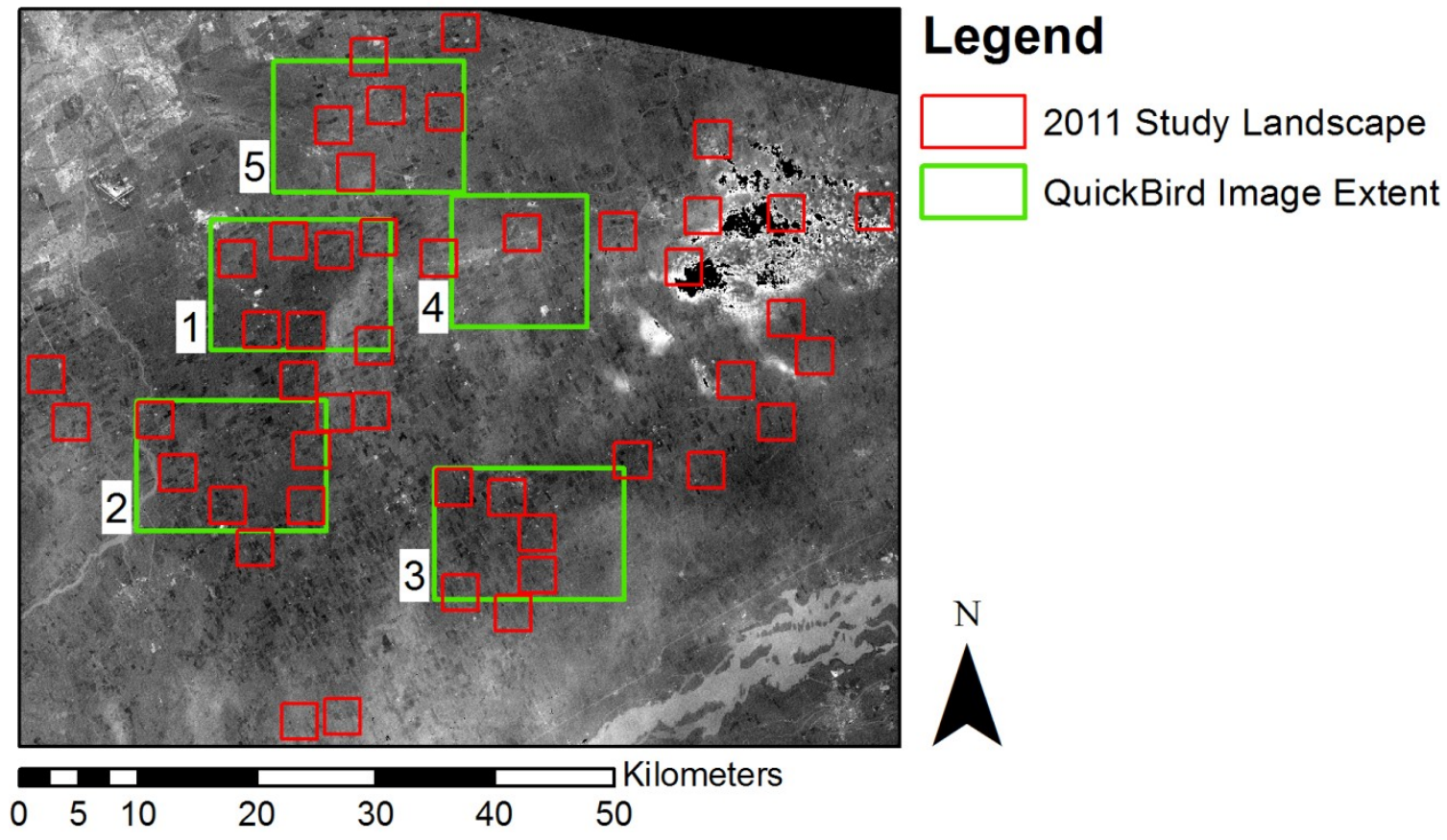

Figure 3.3 Band 4 (haze) of the Tasseled-cap Transformation for the 5 July, 2011, TM image, with numbered QB image extents. Each QB extent is numbered near its lower left-hand corner (numbering based on van den Berg, 2012). Regions of the image that are more affected by haze tend to appear in a lighter tone. 

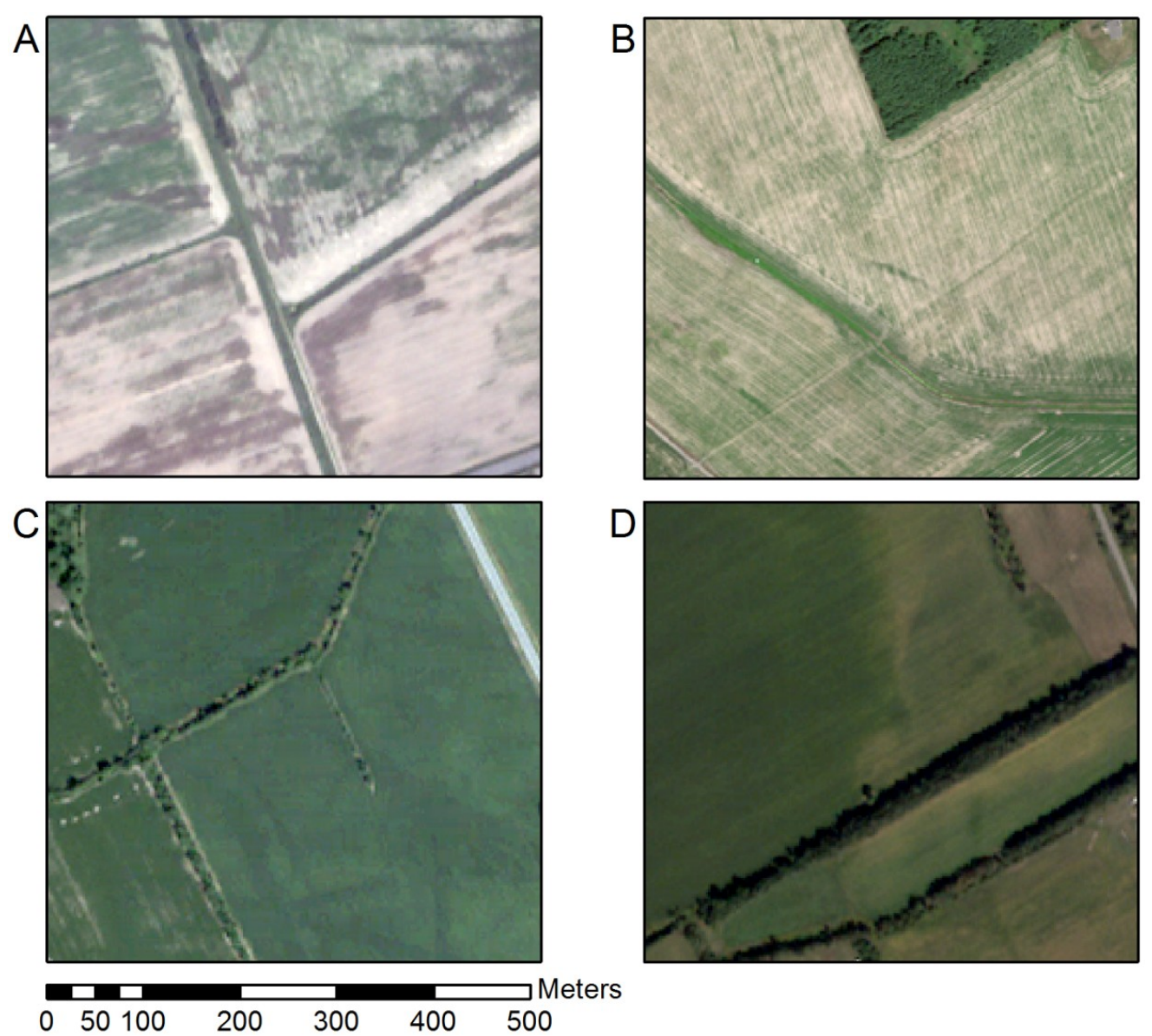

Figure 3.4 Examples of Soybean fields from QB images, including (A) Image 5 (2 July), (B) Image 1 (16 July), (C) Image 3 (12 August), and (D) Image 2 (29 August).

Ultimately, the landscapes that were fully within the extent of QB image 2 were selected. In addition to being within a region of the 5 July TM scene that was less effected by haze (Fig. 3.3), these landscapes spanned three quartiles of the P.AG, MFS, and SHDI distributions for year-2011 (Table 3.3). 
Table 3.3 QB image details, including acquisition dates, number of full study landscape contained, and the proportion agriculture (P.AG), mean field size (MFS), and crop diversity (SHDI) metric quartiles covered by these landscapes (in the year-2011 data).

\begin{tabular}{cccccc}
\hline $\begin{array}{c}\text { QuickBird } \\
\text { image } \\
\text { number }\end{array}$ & $\begin{array}{c}\text { Acquisition } \\
\text { date } \\
\text { (in 2011) }\end{array}$ & $\begin{array}{c}\text { Number of } \\
\text { full } \\
\text { landscapes }\end{array}$ & $\begin{array}{c}\text { Proportion } \\
\text { agriculture } \\
\text { quartiles }\end{array}$ & $\begin{array}{c}\text { Mean field } \\
\text { size quartiles }\end{array}$ & $\begin{array}{c}\text { Crop } \\
\text { diversity } \\
\text { quartiles }\end{array}$ \\
\hline 1 & 16 July & 5 & $1,1,1,1,3$ & $1,1,2,2,4$ & $1,2,3,3,4$ \\
$2^{*}$ & 29 August & 4 & $2,3,3,4$ & $2,3,3,4$ & $1,3,4,4$ \\
3 & 12 August & 4 & $2,2,4,4$ & $1,3,3,3$ & $1,2,2,3$ \\
5 & 2 July & 4 & $1,1,2,2$ & $1,3,4,4$ & $1,2,3,4$ \\
\hline
\end{tabular}

\subsubsection{Classification scheme}

The classes that were considered for this comparison were Corn, Soybean, Hay, Pasture, Legumes, Cereals, Fallow, and Abandoned (Table 3.1). These classes accounted for the majority of the crops in each of the landscape maps, but were not completely exhaustive. Some of the landscapes contained Mixed Vegetable fields (8 small fields in total, with sizes ranging from approximately $5,700 \mathrm{~m}^{2}$ to $18,000 \mathrm{~m}^{2}$ ), Apple Orchards (a single moderately sized orchard of approximately $56,000 \mathrm{~m}^{2}$ ), or fields of an Unknown crop type (Table 3.1). There were a significant number of Unknown fields, particularly in the areas outside of the LCs, where the ground referencing had been performed based on visibility from the nearest public road. 
Since this portion (Section 3.3) of the thesis focuses solely on comparing differences in SHDI distributions that arise due to different simulation approaches, only pixels within the fields that had been ground referenced as Corn, Soybean, Hay, Pasture, Legumes, Cereals, Fallow, or Abandoned were considered. This was done to avoid the effects associated with using a non-exhaustive classification scheme, which are beyond the scope of this comparison.

\subsubsection{Data processing}

From the full (3 km by $3 \mathrm{~km}$ ) landscape maps within QB extent 2 (Fig. 3.3), polygons representing fields that had been ground referenced as one of the crop types considered for classification (including Abandoned) were extracted. These polygons, after processing, were to be used to isolate classified pixels for subsequent SHDI calculations.

There were alignment issues between the QB imagery, the TM imagery, and the field polygons. The TM imagery was offset from the field polygons by roughly 26 meters, but the offset was consistent across all of the TM images, likely due to the systematic geometric correction (Section 3.2.4). Misalignment between the field polygons and the QB image seemed to be more variable, though the offset distance tended to be less than the TM misalignment. In the QB image, some boundaries appeared to be fairly accurately represented with others offset to roughly 10 meters (up to roughly 20 meters in some more extreme cases). 
Rather than attempting to georectify both image sets to the field polygons, the polygons were simply trimmed to the portion of their field that had coverage by both image sets. The northern and western portions of polygons were trimmed to the boundary of the field, as represented by the TM imagery (checked by individual-date natural colour composites and a multi-date NDVI composite, seen in Fig. 3.5 B, D). They were also trimmed to the field boundaries, as represented by the QB image. Areas near the edge of fields that showed large patches of exposed soil or were covered by shadow from trees were also trimmed (Fig. 3.5 A, C).
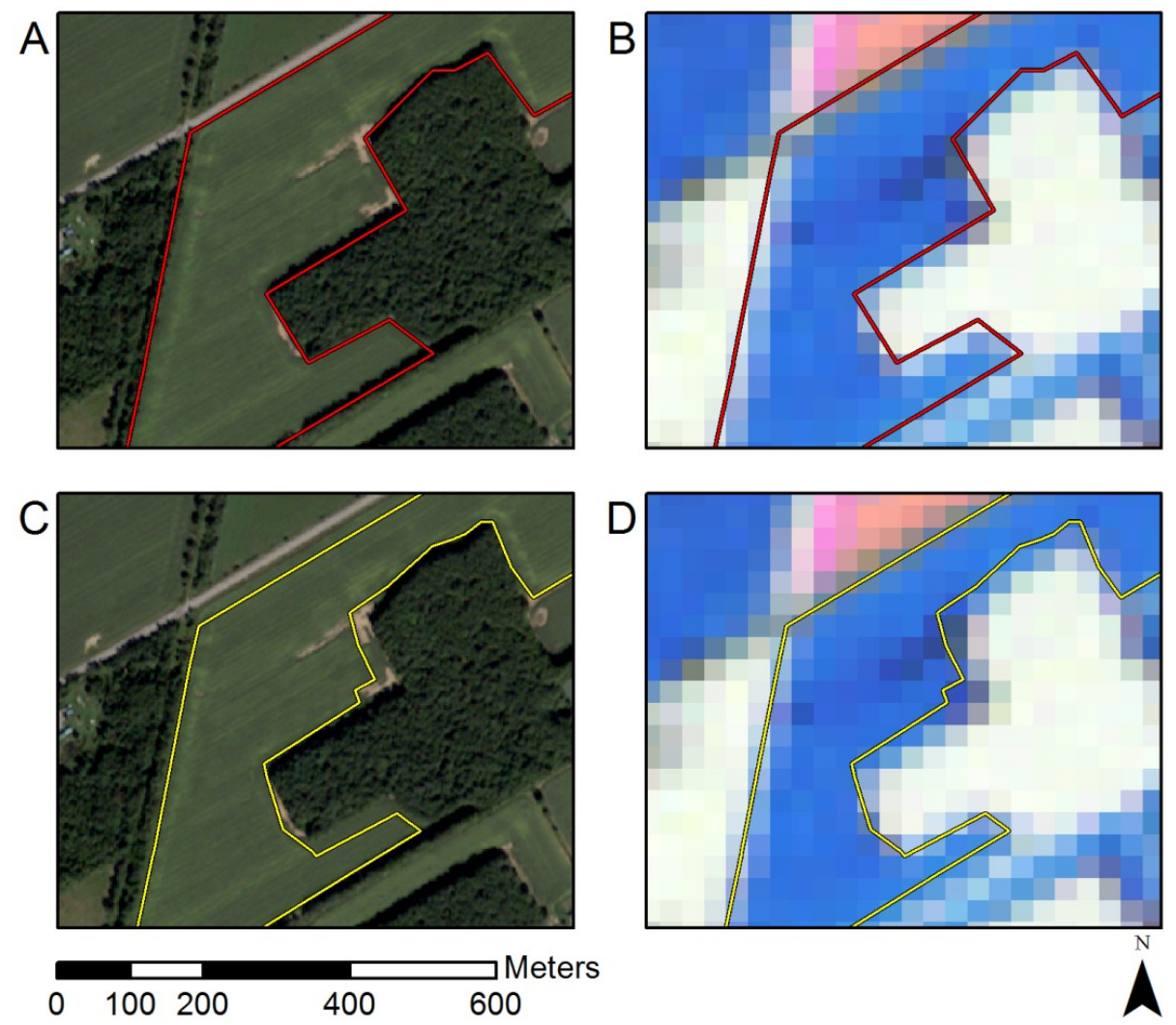

Figure 3.5 Field boundary trimming. The original field boundary (red) is overlain on (A) a QB natural colour composite and (B) a TM multi-date NDVI composite (3 June, 19 June, 5 July). The trimmed field boundary (yellow) is overlain on the same $(\mathbf{C})$ QB, and (D) TM composites. 


\subsubsection{Classifier training areas}

In an attempt to avoid differences between the three image set classifications (Section 3.3.5) resulting from differences in training areas (e.g. different proportions of class training samples, different spatial distributions, and differences in the quality of training area selection), training samples (image pixels) were extracted from a common set of training areas across all image sets.

Training areas were manually delineated, as polygons, within ground referenced fields in the QB image extent. An attempt to limit the amount of training areas within the study landscapes was made, relying mostly on the ground reference data from AAFC (Section 3.2.2), where possible. However, due to limitations with this supplemental AAFC ground referencing set (different Hay-Legumes class definitions and no referenced Fallow or Abandoned fields within the QB extent) some training areas had to be delineated within the landscapes. All Corn and Soybean training areas were outside of the study landscapes (Fig. 3.6). For the other classes (Hay, Legumes, Pasture, Cereals, Fallow, and Abandoned), at least some of the training areas were from within the landscapes (Fig. 3.6). Also, due to limited ground referenced samples, a few training areas for the Fallow and Abandoned classes were delineated based on visual interpretation of the QB and multi-date TM imagery. 


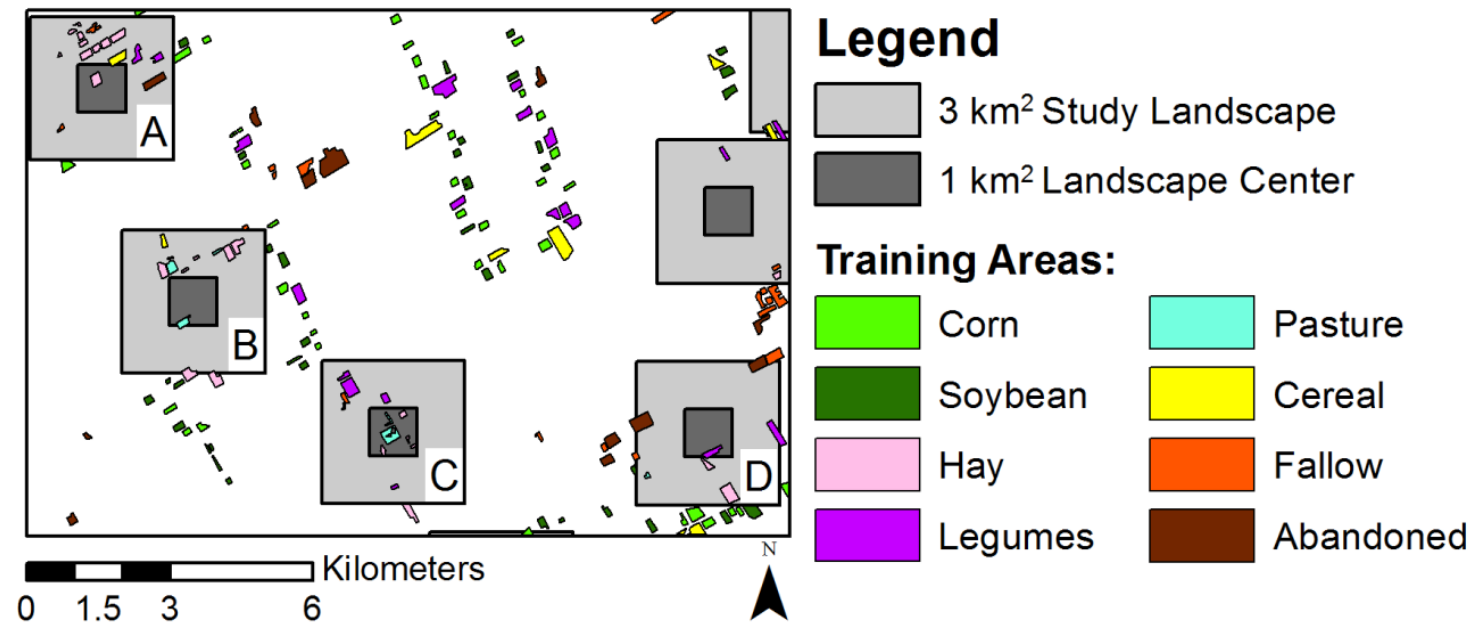

Figure 3.6 Distribution of training areas. The full QB extent is shown, along with the distribution of the study landscapes. The four landscapes that are fully contained within the extent are lettered in the lower right-hand corner.

To account for the fact that the training areas were within the landscapes themselves, classifications were performed separately for each landscape, each time excluding those training samples lying within the landscape being classified. For example, classifications for Landscape A, were performed based on the training areas shown in Figure 3.6, with the exception of those lying within Landscape A.

Delineating enough training areas within the limited extent was problematic for classes other than Corn and Soybean, due to limited ground referenced fields. Due to this, as well as the fact these classes would sometimes have a portion of their training samples removed during any given classification, as many samples as possible (attempting to avoid mixed pixels and cases of questionable ground referencing) were gathered from referenced fields of those limited classes. However, finding enough samples for Pasture remained problematic. The distribution of training samples are shown in terms of pixel counts from TM imagery in Figure 3.7. 


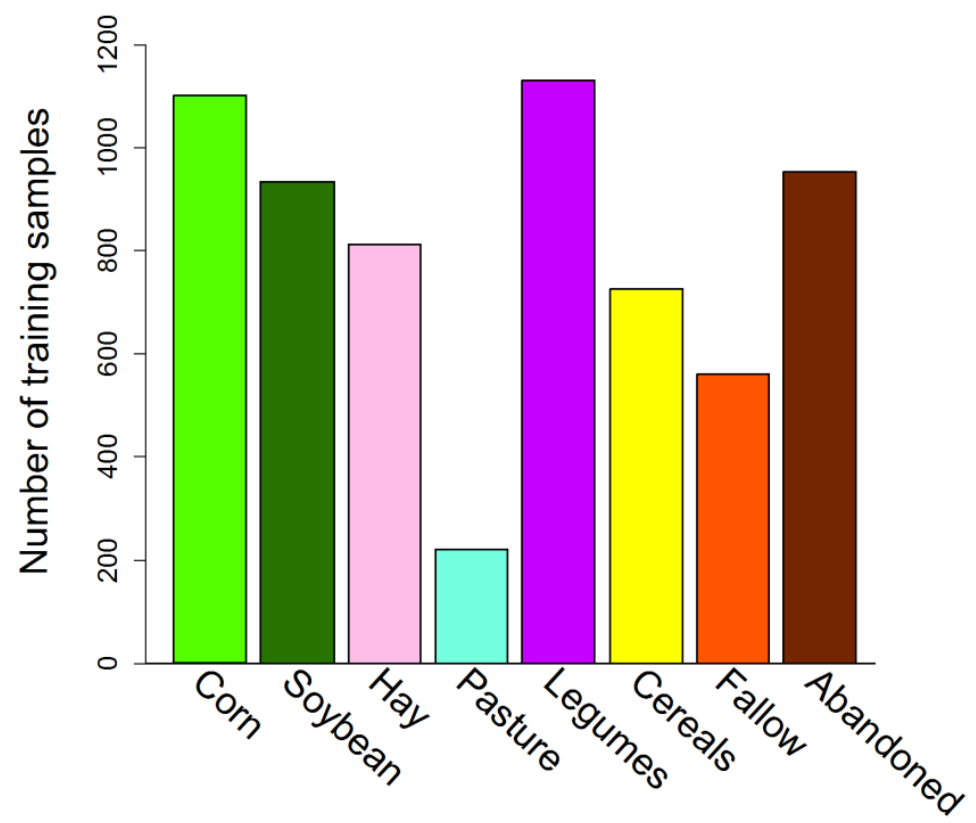

Figure 3.7 Number of TM imagery training samples by class.

\subsubsection{Image sets}

For each of the four landscapes, three separate classifications were performed on different image sets. These image sets were: (1) a single date TM image (19 June), (2) a multi-date composite TM image, and (3) a single date QB image (29 August).

The single date image sets were kept straightforward, comprised of just the image bands, without supplementing the data with band ratios or texture measures. While including additional data may have improved accuracy and reduced uncertainty in some cases, they could have expanded the limited scope of this comparison. Also, since the focus was on the effects of different simulation approaches for uncertainty propagation, having a wider range of uncertainty in the landscape maps was considered an asset to the comparison. 
All image bands (except Band 6) across all of the TM images, including NDVI for each, were initially considered for the multi-date TM image set. Feature selection was performed, so only the 10 most important bands for predicting the crop classes were included in the final multi-date TM image set. These 10 bands were identified by training a Random Forest classifier and ranking the variables in terms of their importance, based on Gini node impurity (Section 2.3.1.2). The 10 selected bands are listed in Figure 3.8.

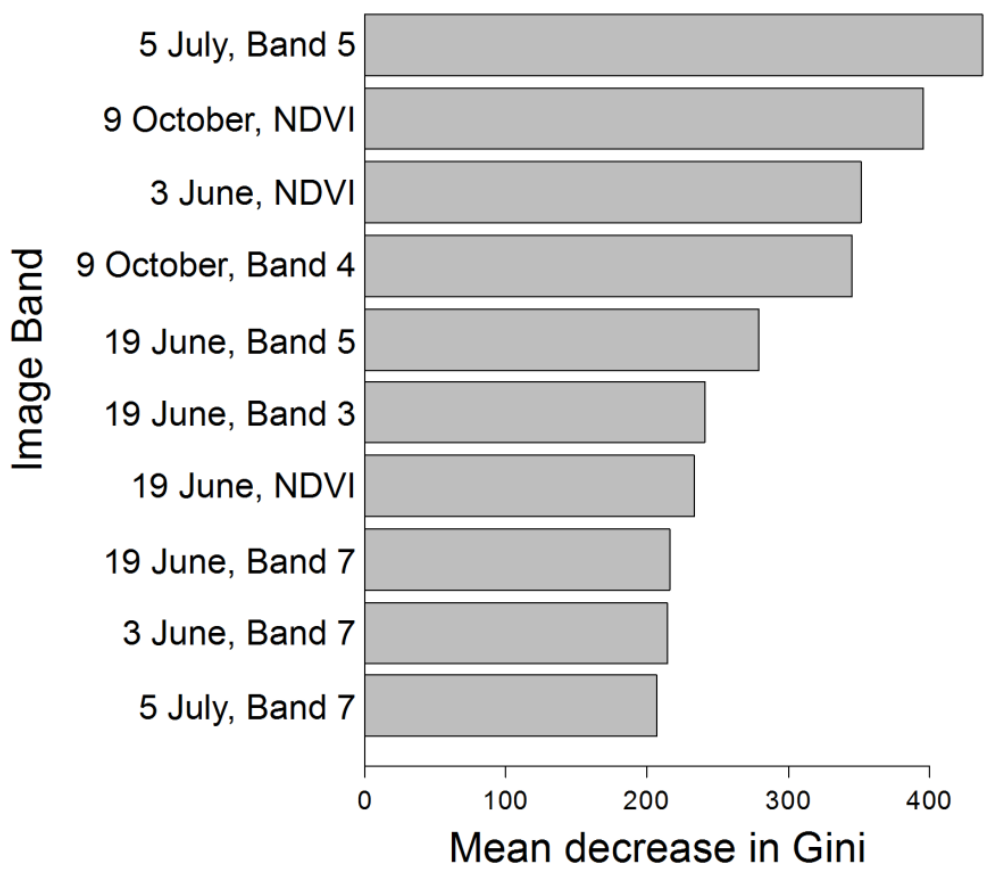

Figure 3.8 Feature selection for the multi-date TM image set. Bands are ordered from largest to smallest mean decrease in Gini node impurity index. Larger mean decreases suggest higher variable importance, based on their ability to separate the data into more homogenous nodes. 


\subsubsection{Training data and classification}

Pixels values within the training areas, across all considered image bands (in a given image set), acted as training data. Many of the training data distributions appeared (visually) to be symmetric and unimodal, though there were cases of multimodality, skewness, and outliers. These cases were more prominent in the longer wavelengths, in classes that were subjected to multiple cuts or grazing throughout the growing season (i.e. Hay, Legumes, or Pasture), as well as in the higher resolution QB imagery (see Appendix B for histograms showing the distributions of training data for some of the imagery).

Class separabilities across the three image sets ranged from poor to good. The average transformed divergence values (a measure of spectral separability between classes) were 1575 (poor) for the QB training data, 1856 (acceptable) for the single date TM training data, and 1988 (good) for the multi-date TM training data (see Appendix C for transformed divergence explanation and values for all class pairs). Corn and Soybean showed somewhat poor separabilities in the single date image sets. The lowest separabilities tended to be between the Fallow and Abandoned classes, as well as amongst the Hay, Pasture, and Legumes classes. While thematically similar classes that exhibit lower separabilities are often merged (Lillesand et al. 2004), they were not in this case. For compatibility with the biodiversity models, and due to the fact that, while spectrally similar, these classes played potentially different ecological functions for certain taxa, these classes were kept separate (accuracy improvements associated with class merging are discussed following the Results). 
A Bayesian classifier, trained on the aforementioned training data, was used to generate per-pixel probabilities of class membership for each of the image set classifications. The classifier was IDRISI Taiga's BAYCLASS classifier, with non-fuzzy clusters defined using the $S I G$ algorithm (as opposed to the fuzzy FUZSIG algorithm) (Eastman \& Laney, 2002). Unlike the Bayesian classifier described in Section 2.3.1.1, the BAYCLASS algorithm had no misclassification cost function, simply outputting posterior probabilities corresponding to those of a softened maximum likelihood classification (Eastman, 2009b; Eastman, 2012). Equal prior probabilities were used because, while crop proportion estimates were available for the scale of the study area, these estimates do not always reflect the crop proportions found at the landscape scale.

For each of the four landscapes, three separate classifications were performed, one for each image set. The output of each of these classifications consisted of 8 rasters (floating point), each representing per-pixel probabilities (ranging from 0 to 1 , summing to 1 across all 8 rasters) of belonging to one of the 8 hard classes. The pixel size and extent of each of these class probability rasters were identical to those of their corresponding image set.

Since the simulation comparison was to only consider those fields that were ground referenced as belonging to one of the 8 classes (as mentioned in Section 3.3.2), pixels corresponding to these fields were isolated prior to simulation. Trimmed field polygons (Fig. 3.5) that had been referenced as one of the 8 classes were converted to a raster mask and used to extract pixels from the class probability rasters (Fig. 3.9 A-C).

In preparation for the homogenous field-based simulation, a version of each of the class probability rasters was produced wherein per-pixel probabilities of class 
membership were aggregated to the field level. For each class probability raster, this was done by taking the zonal mean of all pixels within the same field, with unique fields identified by a raster mask (trimmed field polygons converted to raster, with unique identifiers for each field, as shown in Fig. 3.9 B). While these aggregated, per-field probability rasters maintained the same pixel size and extent as the per-pixel probability rasters, all pixels within the same field were now identical (identical PDFs), still ranged from 0 to 1 , and still summed to 1 across all 8 rasters (Fig. 3.9 D).

In total, there were 24 classification sets (4 landscapes $\times 3$ image sets $\times 2$ classification representations: per-pixel and per-field), each set containing 8 probability rasters (8 classes). 

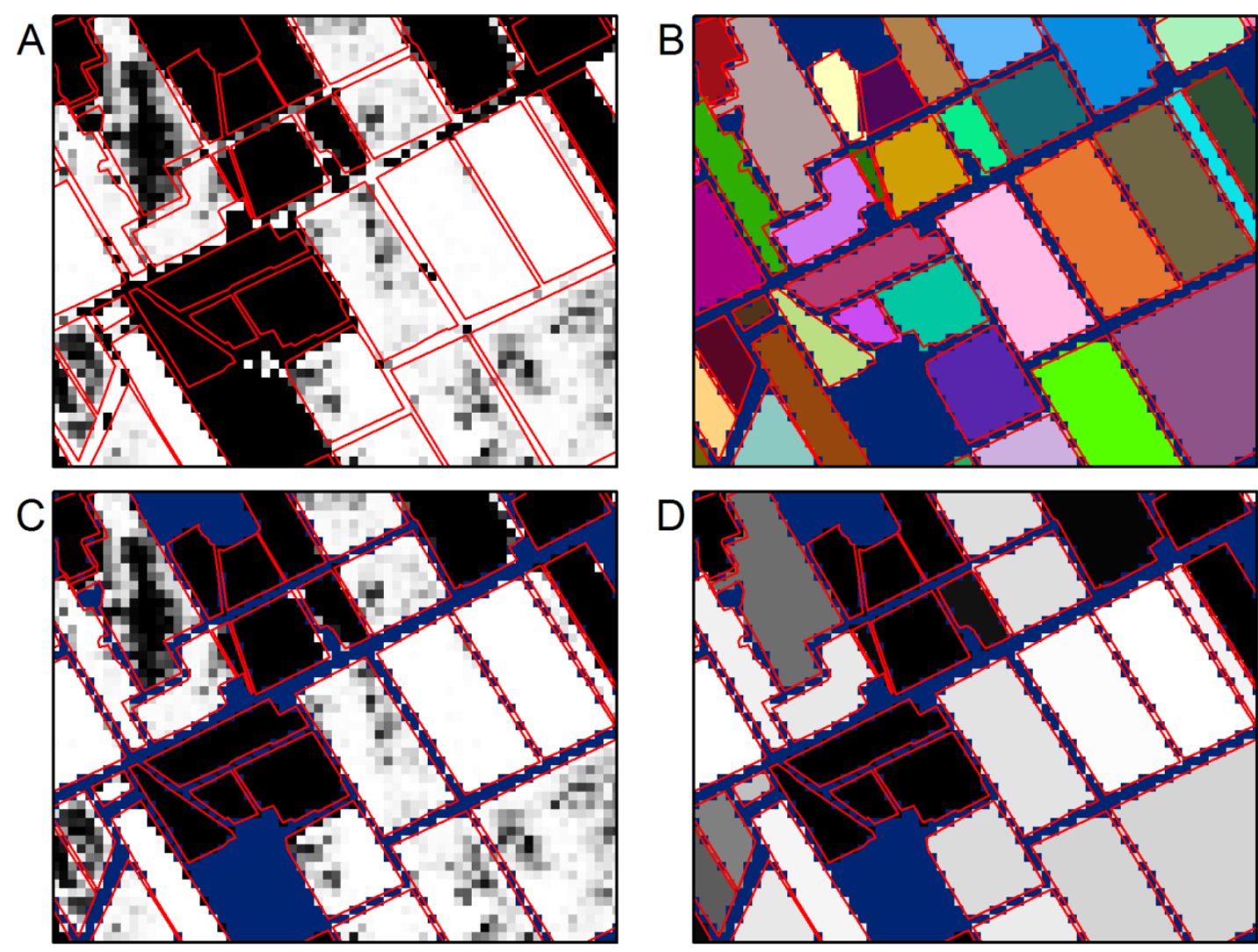

Figure 3.9 Class membership probability raster processing. Example shows

(A) a class probability raster (trimmed field polygons shown in red), (B) trimmed field polygons converted to a raster mask with unique identifiers for each field, (C) extracted per-pixel probabilities of class membership, and (D) per-field probabilities of class membership. Dark blue background represents 'no data' pixels.

\subsubsection{Simulation}

As described at the beginning of Section 3.3, three different MC simulation approaches were applied to each classification set. The independent pixel-based and field-based approaches both used the per-pixel probability rasters (Fig. 3.9 C) while the homogenous field-based simulation approach used the per-field probability rasters (Fig.

3.9 D). Prior to simulation, these probability rasters were converted to cumulative 
probability rasters (representative of the CDFs for each pixel) in the order: (1) Corn, (2) Soybean, (3) Hay, (4) Pasture, (5) Legumes, (6) Cereals, (7) Fallow, and (8) Abandoned. This was done by cumulatively summing the probability rasters $($ i.e. $1 ; 1+2 ; 1+2+3$; $1+2+3+4$; and so on).

For the independent pixel-based simulation approach, an independent PRN was generated for each pixel (independent of the PRNs of nearby pixels). These PRNs were then used to sample the cumulative, per-pixel probability rasters (representing CDFs), assigning a simulated class to each pixel (Section 2.5.1).

For the field-based simulation approach, a common PRN was generated for all pixels within the same field, with fields defined by the same unique-field raster mask as was used for producing the per-field probabilities (Fig. 3.9 B). As before, these PRNs were then used to sample the cumulative, per-pixel probability rasters (representing $\mathrm{CDFs}$ ), assigning a simulated class to each pixel.

For the homogenous field-based simulation approach, a common PRN was generated for all pixels within the same field, as was done for the field-based simulation approach. However, unlike the field-based simulation approach, these PRNs were used to sample the cumulative, per-field probability rasters (representing CDFs). Because both the PRNs and class probabilities were homogenous within fields, there was no opportunity for within-field variability in terms of simulated class.

For a given classification set and simulation approach, once all pixels had been assigned a simulated class, the SHDI of the resulting landscape realization was calculated (Section 2.6) and stored. All classes, except for Abandoned, were considered in the SHDI calculation. This process of generating a realization and calculating SHDI was 
repeated a total of 1000 times, for each classification set and simulation approach. With each repetition, variance in SHDI across all previously generated realizations (inclusive of the current realization) was calculated and stored. These cumulative variance records were used to check that the 1000 realizations was sufficient for a reasonable degree of convergence on an overall variance estimate (Section 2.5.1).

\subsection{Effects of positional and thematic uncertainties on the model coefficients}

In order to quantify the effect of positional and thematic uncertainties in the LC maps on the relationships established in the biodiversity models, relatively simple MC simulation approaches were used. These involved generating realizations of all LC maps in the study area, based on positional uncertainty estimates (for LC maps from both years) and thematic uncertainty estimates (for the year-2011 LC maps only). Only the LCs were considered (as opposed to the full $3 \mathrm{~km}$ by $3 \mathrm{~km}$ extent maps), as the biodiversity models were based on metrics from these LCs (Section 2.6). The methods presented in this section are aimed at addressing research questions 2 and 3 (Section 1.3), involving the analysis of positional and thematic uncertainties, and their influence on model coefficients.

Positional errors and uncertainties associated with digitizing can be assigned global representations through, for example, the epsilon band model or probabilistic representations via normal distributions centered on vertices (Shi et al. 1999; Fortin \& Edwards, 2001; Section 2.4.2). While global estimates can represent the random error associated with vertex positioning along an interpreted boundary (Shi et al. 1999), the 
influence of local contextual information can be important to consider in some instances (Edwards \& Lowell, 1996). Since the landscapes studied here have a very discrete spatial structure (i.e. discrete as opposed to gradual changes in land covers), coupled with varying levels of field boundary interpretability (features constituting field boundaries were very clear in some cases, while unclear in others), positional uncertainty was broken down into two components:

(1) the global uncertainty associated with vertex positioning along an interpreted boundary, and

(2) the local, field-level uncertainty associated with identifying features that constitute the field boundary.

An example of these two components is shown in Figure 3.10, below.
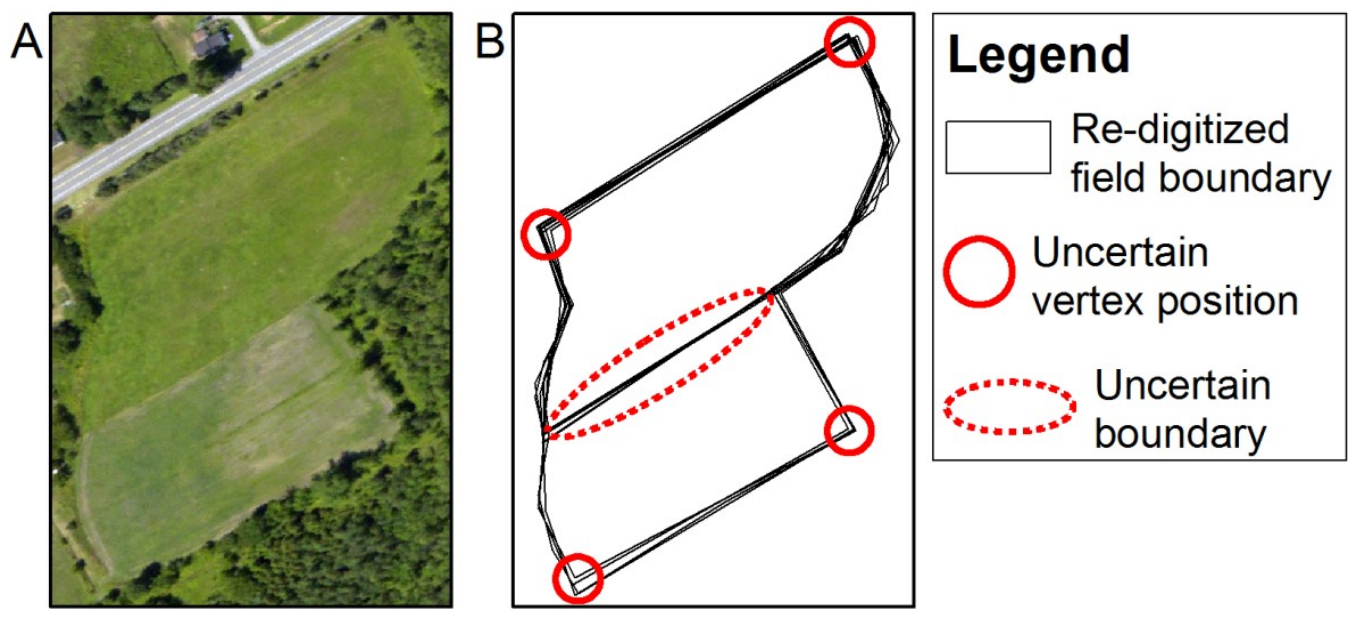

Figure 3.10 Example of positional uncertainties. The example shows (A) a field (or fields), as seen in a high resolution air photo, with (B) examples of multiple re-digitized boundaries for the field. With digitizing beginning from the top of the image, in some cases the lower portion is considered to be a separate field. 
The original biodiversity models used SHDI metrics from ground referenced LC maps (Section 2.6). In order to consider the effects of thematic uncertainties, this portion of the thesis adopts a scenario where only the year-2012 crop types had been ground referenced. The year-2011 crop classes had, instead, been assigned based on the classification of the TM imagery. Therefore, the propagation of thematic uncertainties was simulated for the year-2011 LC maps, but not for the year-2012 LC maps (thematic uncertainties in ground referenced crop types assumed to be negligible). It should be noted that, due to this adopted scenario, the biodiversity models being analyzed here differ from the original models provided by the Farmland Biodiversity Project, in terms of the data that they were based on.

\subsubsection{Positional uncertainty estimation: Vertex position uncertainty}

Uncertainty in vertex positioning, along features that were assumed (via air photo interpretation) to represent the true boundaries of a field, was estimated based on vertex matching across a sample of fields, that had been re-digitized (Section 2.4.2).

A subset of 100 fields from the year-2011 landscapes (not limited to just LCs) was generated. Fields were selected based on random sampling, stratified by crop type and field area (quartiles). Only full fields were considered (i.e. fields intersecting the 3 $\mathrm{km}$ by $3 \mathrm{~km}$ boundaries were left out). In cases where a selected field did not have several distinct corners (ideally four perpendicular corners), another field of the same crop type and similar field size (adjacent in a list of fields, sorted by crop type and field area) was selected in its place. 
The subset was re-digitized 24 times, 12 on the year-2008 air photo and 12 on the year-2011 air photos. While this was performed by an individual digitizer, attempts were made to reasonably vary the approach across reproductions. The scale was varied from 1:2000 to 1:5000. For some reproductions, the field was digitized directly, while in others the edges surrounding the field were digitized, thereby indirectly digitizing the field itself. Some reproductions were performed with slightly more or less willingness to adjust for tree canopy overhang along field boundaries. Finally, attempts were made to be more slow and deliberate with vertex placement in some reproductions, and more hasty in others.

For each field in the subset, two to four somewhat visible corners (ideally close to perpendicular) were selected based on availability. A total of 364 were selected across the subset. At each of these corners, the North-South and East-West distances between the re-digitized vertices and the reference vertex were recorded. However, in some cases, not all 24 re-digitized vertices at each corner were compared to the reference vertex. For some fields, the two air photos were not highly aligned. In cases where there was a clear offset between the re-digitized vertices from the two air photos, only those vertices that were re-digitized on the photo that most-closely matched the reference vertex were used (recall that the reference digitizing had been based on a combination of the year-2008 air photo and the year-2011 or year-2012 air photos; Section 3.2.1). In other cases, there was some boundary interpretation uncertainty in the re-digitized vertices, resulting in some vertices being far offset from the others. The far-offset vertices were omitted. These cases are illustrated in Figure 3.11, below. 

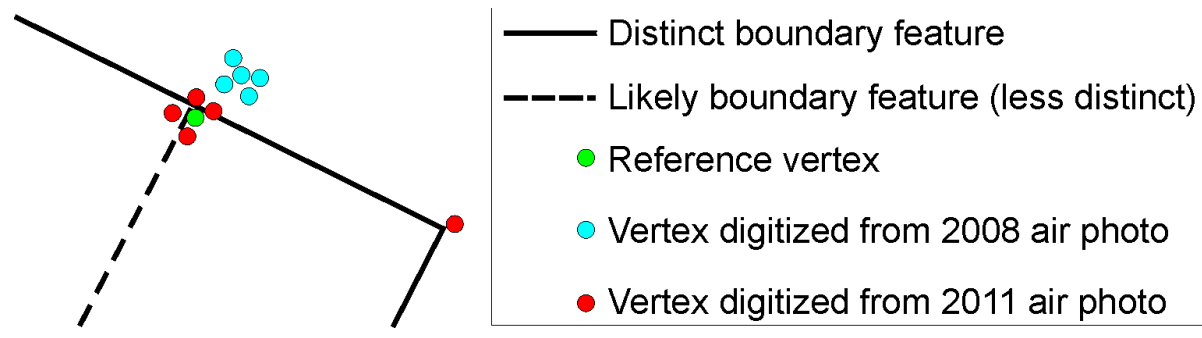

Figure 3.11 An illustrated example of cases where vertices were omitted from the vertex position uncertainty estimation. The reference vertex, here, was likely digitized on the year-2011 air photo, so the year-2008 vertices would not be considered. The year-2011 vertex on the far right would not be considered, as it falls under boundary interpretation uncertainty.

The overall distributions of distances between the reference and re-digitized vertices at the 364 corners were fairly normal (Fig. 3.12, below), with East-West and North-South standard deviations of $3.89 \mathrm{~m}$ and $4.39 \mathrm{~m}$ respectively. Vertex position uncertainty was therefore represented by normal PDFs in the East-West and North-South directions, with these standard deviations, centered on each reference vertex position.
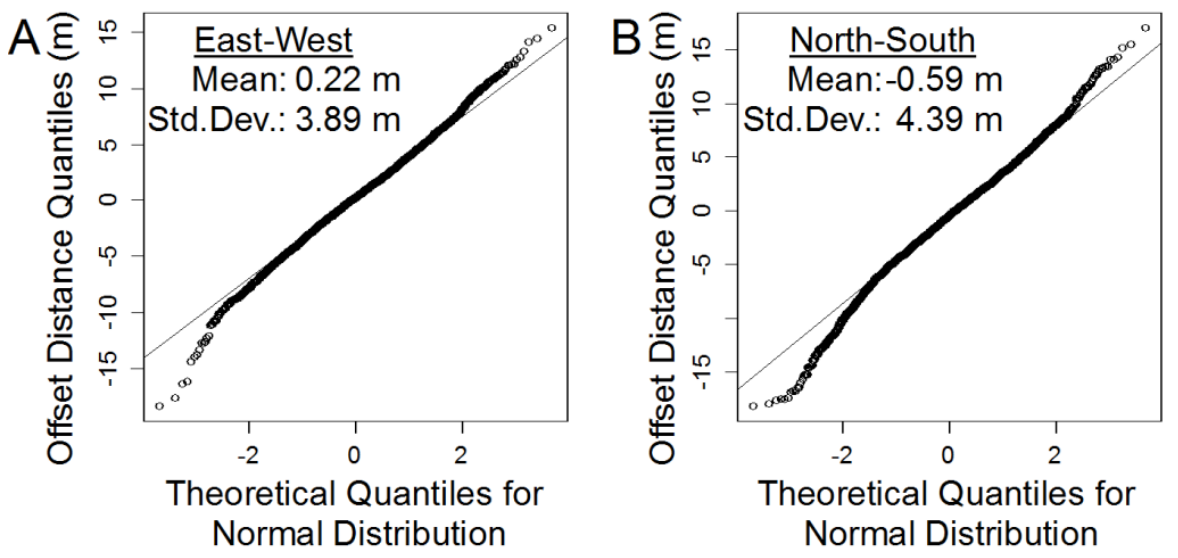

Figure 3.12 Quantile-quantile plots comparing the offset distances between reference and re-digitized vertices to the normal distribution, in the (A) East-West and (B) North-South directions. There were 4327 matches between re-digitized and reference vertices, across the 364 corners. 
To confirm that there were no major differences in positional uncertainty between the year-2011 and year-2012 LC maps, one field from each study landscape was randomly selected (one of the year-2012 landscapes was omitted due to not having air photo coverage) and re-digitized once, at a scale of 1:2000, on the respective year-2011 or year-2012 air photos. North-South and East-West offset differences between the redigitized and reference vertices were recorded at two corners in each of these selected fields. Bartlett tests, for testing the equality of variances between normally distributed populations (null hypothesis of equal variances) (Arsham \& Lovric, 2011), were used to compare the North-South and East-West variances associated with the year-2011 vertices, to those of the year-2012 vertices. The test did not find a significant difference $(\alpha=0.05)$ in variance between the year-2011 and year-2012 vertices for either the North-South and East-West variances $\left(p_{\text {East-West }}=0.325 ; p_{\text {North-South }}=0.822\right)$. Therefore, vertex position uncertainty for the year-2012 LC maps was represented with the same PDFs (centered on different vertices) as the year-2011 LC maps.

\subsubsection{Positional uncertainty estimation: Boundary interpretation uncertainty}

The uncertainty associated with interpreting which landscape features constitute the boundary of an individual field (Fig. 3.10) was estimated locally, based on the visibility of a potential edge and contextual factors (e.g. shape and position of the edge, texture or patterns on either side, and colour on either side). Cases where this uncertainty was considerable were identified through interpretation of the year-2011 or year-2012 air photos (not considered for one of the year-2012 LCs that did not have air photo 
coverage). These fields were duplicated, and the duplicates were manually merged (Fig. 3.13 A, B) or split (Fig. 3.13 C, D) as necessary to represent the alternate possible field boundaries. Additionally, there were some cases where field boundaries (or portions of a field's boundaries) were taken out of, or entered into, consideration in alternate representations (Fig. 3.13 E, F).

In total, 339 of the 1916 fields within the LCs were given at least one alternate possible representation. In preparation for simulation, fields or groups of fields that shared an alternate boundary representation were all assigned a unique group identifier. Within each group identifier, all alternative representations were further assigned a unique alternate identifier, each of which were assigned probability estimates of being the true boundaries of the field.

It should, again, be noted that edges within the LCs had been checked by ground referencing teams (Section 3.2.1). However, the scenario adopted by this thesis involves minimal ground referencing within the LCs (except for the year-2012 crop types, due only to a lack of imagery for classification). Additionally, even with ground referenced edges, there were some cases of uncertainty associated with either possible error on part of the ground referencing and digitizing teams or changes in boundaries throughout the growing season. In addition to the cases shown in Figure 3.13, below, some examples are provided in Appendix D. 

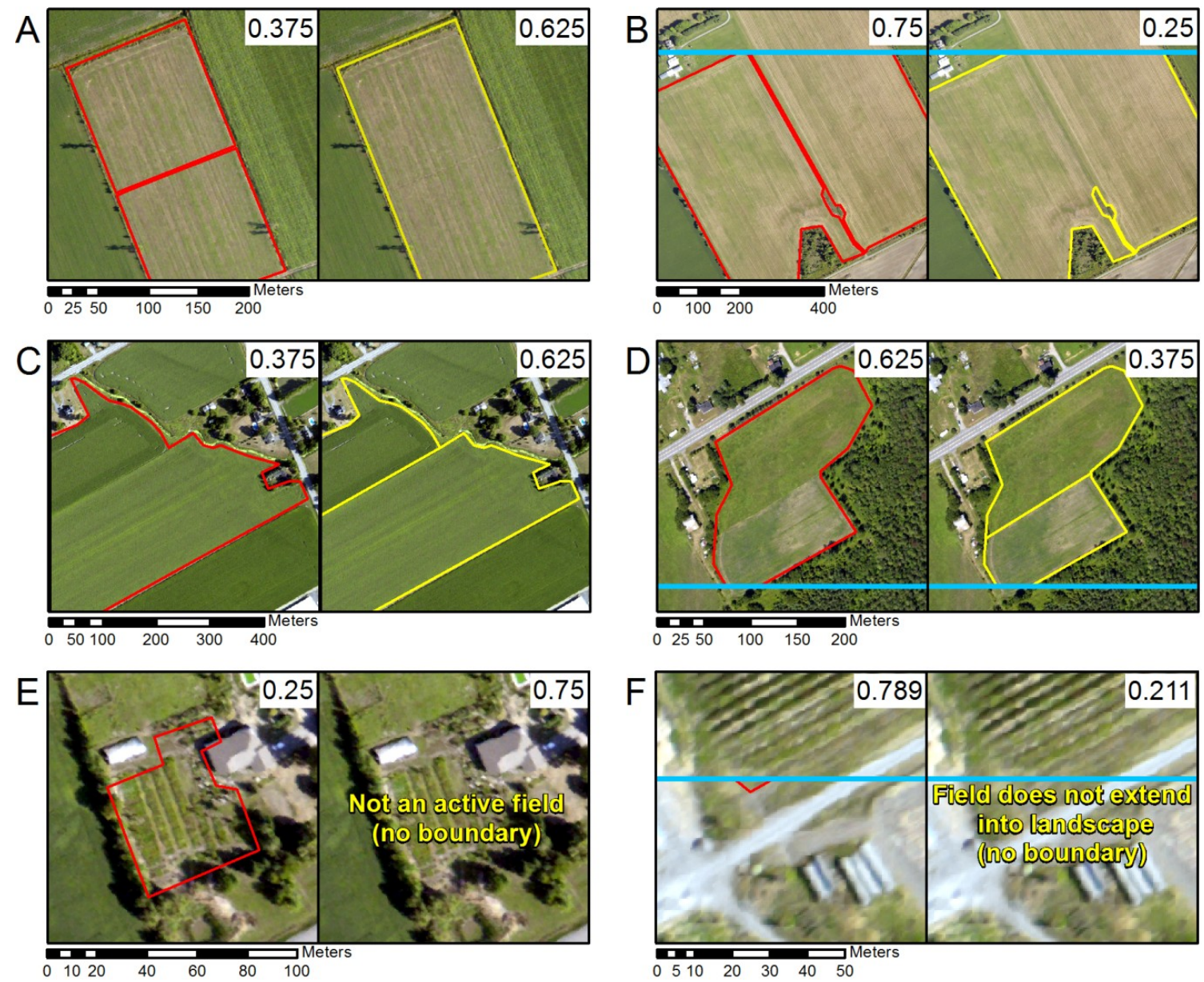

Figure 3.13 Examples of local estimates of boundary interpretation uncertainty, with reference fields (red; left pane) and alternate boundaries (yellow; right pane). Examples include (A, B) merges, $(\mathbf{C}, \mathbf{D})$ splits, and $(\mathbf{E}, \mathbf{F})$ removal of a boundaries from consideration. The landscape border appears in blue, and probability estimates are shown in the top right.

\subsubsection{Merge and split cases}

For potential merged or split alternatives to a reference field, a Canny edge detector was used to provide estimates of edge visibility. The year-2011 and year-2012 air photos were converted to greyscale, smoothed by a 3 pixel by 3 pixel Gaussian filter, and were subjected to three separate runs of a Canny edge detector, with varying 
detection thresholds. Lower:upper thresholds for the detection runs were 20:30, 30:60, and 40:80. These thresholds were determined through trial and error, attempting to roughly match what a human interpreter might consider a low, medium, or highly visible feature.

Merged or split alternatives were assigned probability estimates based, in part, on the proportion of the edge that was detected by the edge detector. Up to $25 \%$ probability in favour of a merge or split of a reference field could be assigned from each of the three binary edge maps produced by the three edge detector runs. For potential merges:

- $25 \%$ in favour would be assigned if 0 to $1 / 3$ of the length of the edge was detected,

- $12.5 \%$ if $1 / 3$ to $2 / 3$ of the length was detected, and

- $0 \%$ if $2 / 3$ to the full edge length was detected.

Conversely, for potential splits:

- $25 \%$ in favour would be assigned if $2 / 3$ to the full edge length was detected (Fig. $3.14 \mathrm{~A})$

- $12.5 \%$ if $1 / 3$ to $2 / 3$ of the length was detected (Fig. $3.14 \mathrm{~B}$ ), and

- $0 \%$ if 0 to $1 / 3$ of the length was detected (Fig. $3.14 \mathrm{C}$ ). 

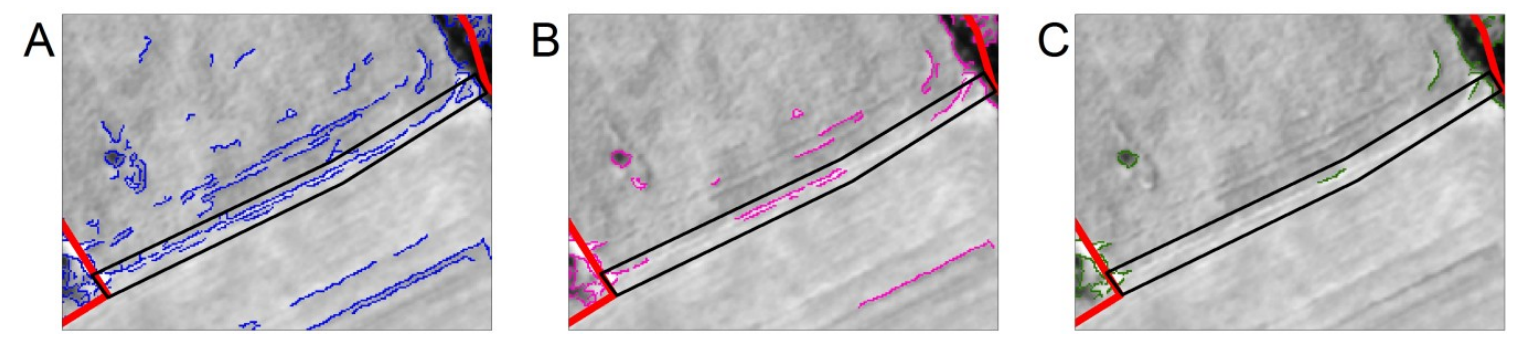

Figure 3.14 Examples of the results of three Canny edge detector runs at (A) lower thresholds (lower visibility edges detected), (B) medium thresholds (medium visibility edges detected), and (C) high thresholds (only high visibility edges detected). A black polygon surrounds the potential split being considered. The background is the greyscale, Gaussian-filtered air photo.

Due to time constraints, the proportions for each edge were not measured in cases where it was clear which interval it fell into (e.g. Fig. 3.14 A, C), though unclear cases were measured manually (e.g. Fig. 3.14 B).

In total, the three binary edge maps accounted for up to $75 \%$ of the probability assignment. The remaining $25 \%$ was assigned in favor of the reference representation, as there was at least some evidence supporting it, in order to be digitized as such, and the original digitizer likely had a strong familiarity with the landscape at the time. Finally, since there were many factors that were not considered by the edge detector (e.g. colour, field texture, crop row direction, etc.), as well as the fact that it could be sensitive to shadow or exposed soil, leeway of $\pm 25 \%$ probability ( $12.5 \%$ increments) was assigned based on interpretation alone. There were some rare cases where more than $\pm 25 \%$ leeway was given. These were cases where there appeared to be fairly clear errors in the reference boundaries, such as permanent structures (i.e. roadway, or a house) lying within field boundaries (Appendix D). 


\subsubsection{Agricultural versus non-agricultural boundary cases}

As seen in Figure 3.13 E, some alternates involved the addition or removal of the field boundary from consideration. Less common than field merges or splits, these were typically cases where it was unclear whether a field was Fallow versus Abandoned, or a small, freshly-cut Hay field versus a lawn or a well-maintained non-agricultural field. While the uncertainty associated with these cases is related to thematic uncertainty, since the distinction between many agricultural and non-agricultural areas was mainly based on photo interpretation during digitizing (some were done during the ground referencing), these cases were considered under the boundary interpretation uncertainty approach.

For cases where an alternate representation involved removing a boundary from consideration, the record for the reference field, within the GIS dataset, was duplicated without the geometry of the field itself. In the duplicate record, the area of the field was set to zero. Probability estimates for these cases were based on interpretation alone, checking the air photos, the QB imagery (when coverage was available), and multi-date TM imagery composites.

Cases where an alternate representation involved adding a boundary for consideration were handled similarly. For these cases, the potential boundary was digitized, assigned a referenced crop type of 'Unknown', and its area calculated. To represent the fact that it may not be a field, a duplicate record with no geometry and an area of zero was created. Probabilities, again, were based on interpretation alone. 


\subsubsection{Sliver cases}

As seen in Figure $3.13 \mathrm{~F}$, there were some cases that involved the removal of some very small area (sliver) corners of fields that only slightly extended into LC. While having little effect on P.AG and SHDI, the small area of these slivers could be a significant outlier in the calculation of MFS. These cases were handled in a similar fashion to the removal of boundaries from consideration for agricultural versus nonagricultural fields (Section 3.4.2.2); however, the assignment of probabilities was handled differently.

For these cases, the probability of removal was calculated as the probability that the corner vertex actually extends past the border, given the vertex positional uncertainty PDFs (Section 3.4.1). The North-South or East-West distance from the border of the LC to the vertex that extended the furthest into the LC was measured. A one tailed Z-test based on this distance relative to the appropriate vertex positional PDF (the North-South PDF if the vertex was just North or South of the LC border, or the East-West PDF if the vertex was just East or West of the LC border; Section 3.4.1) was then used to determine the probability.

\subsubsection{Thematic uncertainty estimation}

Crop types for the year-2011 LC maps were based on image classification. The thematic uncertainty associated with crop class assignment was estimated at the field level (per-field), under the assumption that fields were thematically homogenous (recall the aggregation of per-pixel probabilities to the field level, that was performed earlier: 
Fig. 3.9). Prior to classification, the TM imagery was shifted to better align with the reference field boundaries. Training areas were then delineated and estimates of class probability were obtained from a Random Forest classifier.

\subsubsection{Thematic Mapper imagery processing}

Due to the somewhat consistent nature of the TM imagery offset from the reference field boundaries, North-South and East-West linear shifts were applied to the imagery (all four dates) to better align it. After converting to the TM images into the same spatial reference system as the landscape maps (this conversion from the World Geodetic System of 1984 to the North American Datum of 1983 made very little difference in terms of alignment), four distinct field corners within each of the year-2011

landscape maps ( $3 \mathrm{~km}$ by $3 \mathrm{~km}$ extent) were matched to the corresponding corners as they appeared in the TM imagery (multi-date composite). Based on the mean offsets across all matched corners, the imagery was shifted 20.6 meters North and 16.5 meters West. Following the shift, the RMSE was 8.56 meters in the North-South direction and 8.19 meters in the East-West direction.

Areas of cloud cover and heavy haze in the 5 July, 2011, TM image were manually delineated and removed from the scene via a raster mask. Four of the year2011 LCs fell completely within the cloud mask and two others fell partially within it (Fig. 3.15). 


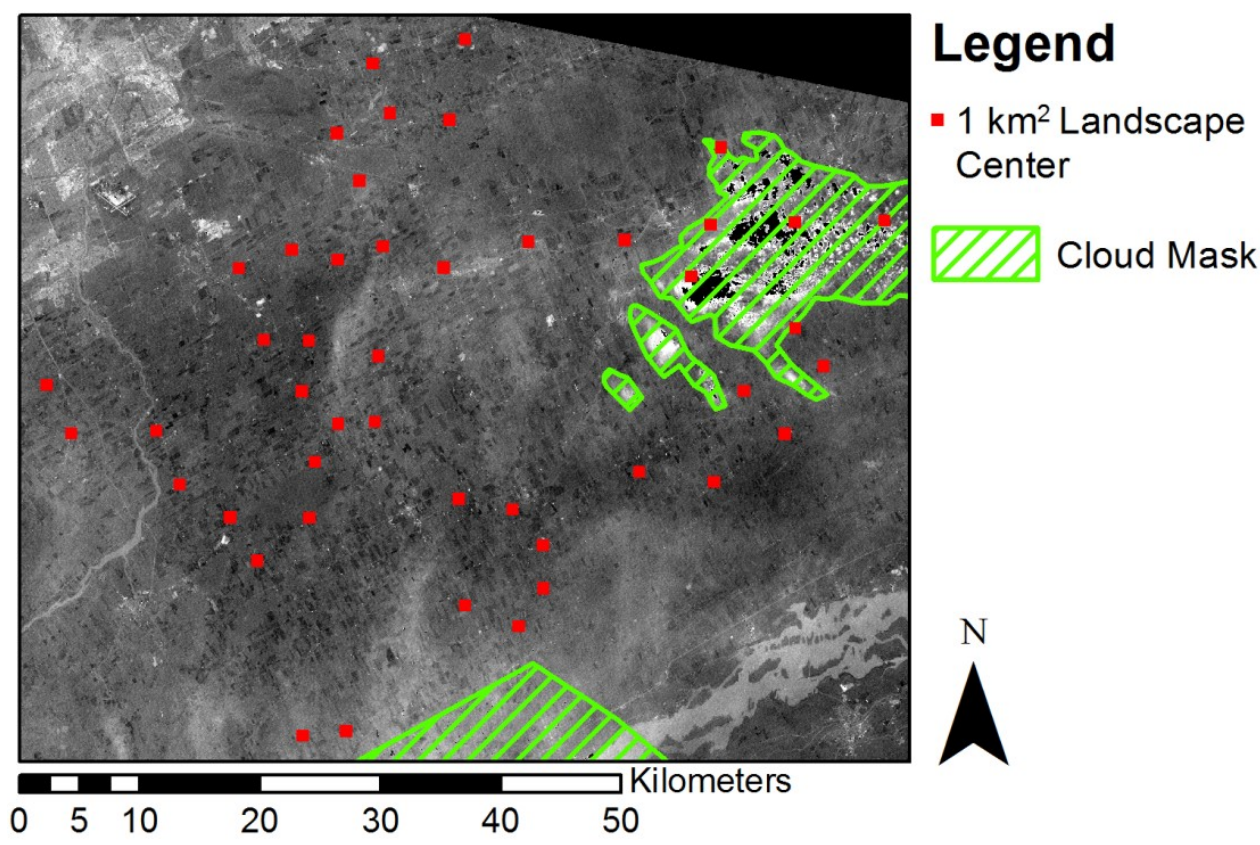

Figure 3.15 Locations of the year-2011 landscape centers relative to the cloud mask. The background image is a haze map (haze appears in a lighter tone) based on Band 4 of the Tasseled Cap transformation. The vector mask shown here was converted to raster and used to remove regions of cloud from the image.

\subsubsection{Classification scheme and training samples}

Agricultural land covers considered for the classification were: (1) Corn, (2) Soybean, (3) Hay, (4) Pasture, (5) Legumes, (6) Cereals, and (7) Fallow (see Table 3.1 for class definitions). Training areas for these classes were delineated in fields within the landscapes, excluding the LCs, as well as in referenced fields from the AAFC dataset (Section 3.2.2). The total number of training samples (pixels) within these training areas are shown in Figure 3.16, below. 


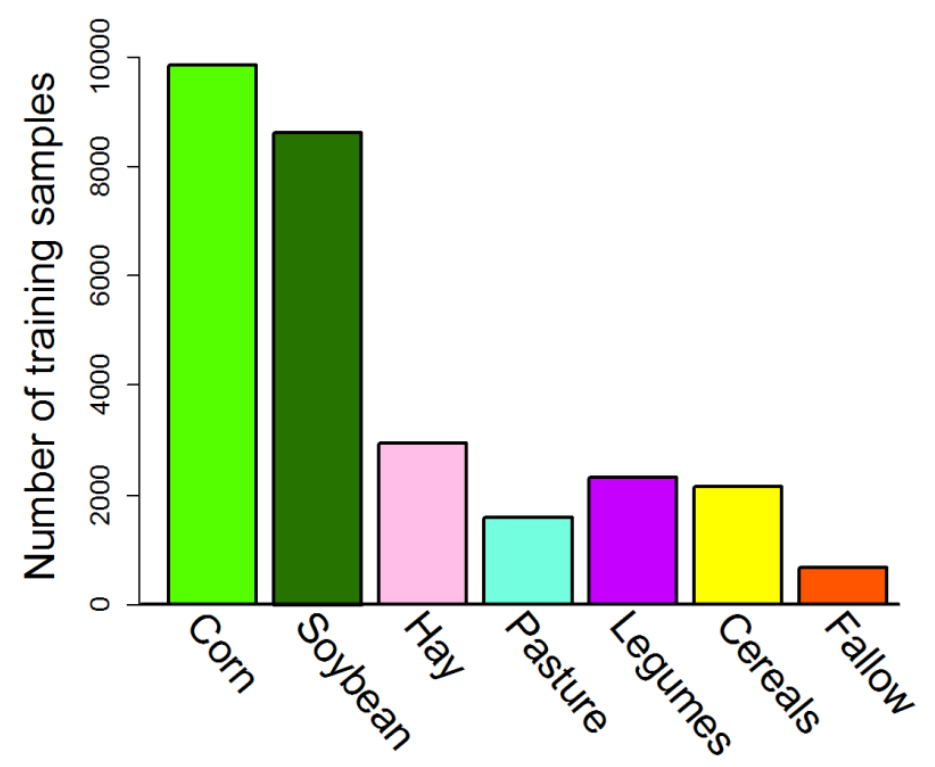

Figure 3.16 Number of training samples, by class, for the year-2011 study area classification.

The training data included all TM bands from the four acquisition dates, except for Band 6. NDVI from each date was also included (Section 3.2.4). A separate set of training data, excluding the 5 July bands, was produced for the classification of pixels within the 5 July cloud mask (Fig. 3.15).

The training data distributions appeared (visually) to be symmetrical and unimodal in some cases, though there were also several cases of skewed and multimodal distributions. Multimodality often occurred in the longer wavelength bands for classes like Hay and Legumes, which tend to be cut at various times throughout the growing season. Histograms of the training data values across the four TM images, as well as a map showing the distribution of the training samples, are included in Appendix E. 


\subsubsection{Classification}

For the full advantages of OBIA, the features that are to be identified and classified should be should be significantly larger than the pixel size (Blascke, 2010). In the landscapes studied here, there were many cases where separate but spectrally similar land covers (the same crop type in some instances) were separated by an edge that was quite thin (e.g. less than 5 meters) relative to the pixel size. Additionally, there were cases of very small fields or within-field changes in crop condition (an example can be seen in Fig 3.4 D). Because of these cases, OBIA based on the TM imagery alone would likely have resulted in a landscape structure that differed significantly from that of the reference LC maps. Therefore, a pixel-based classification approach was used instead, in conjunction with the manually digitized field boundaries.

To determine a reasonable classification approach, a series of trial classifications were performed using the training data outlined in Section 3.4.3.2. Maximum likelihood trial classifications were performed, as well as Random Forest classifications as a nonparametric alternative. Trial maximum likelihood classifications had an overall, per-pixel accuracy of $63.5 \%$, and $69.1 \%$ when the probabilities of class membership were aggregated to the field level (using the same approach as in Section 3.3.6) and hardened (most-likely class assigned). Trial Random Forest classifications, on the other hand, had an overall per-pixel accuracy of $75.3 \%$, and $84.8 \%$ when aggregated to the field level. The decision to use a Random Forest classifier was made, due to the good trial results. Additionally, a comparison of classification approaches in Duro et al. 2012, had showed 
good overall accuracies with pixel-based Random Forest classifications, comparable to other common non-parametric classifiers: decision trees and support vector machines.

Random Forest classifiers of 500 classification trees, the default number, were trained on the same data as the trial classifications. Separate Random Forests were trained for classifying areas outside of the cloud mask, versus those within it (July 5 TM image omitted for those within the cloud mask). The number of variables randomly selected for each split $(m)$ was the square root of the total number of included bands $(m=$ 5), again default. Equal class weights were used, and the number of samples included in each bootstrap sample was equal to the number of original training samples. Attempts at changing the number of trees (numbers of 1000 and 300 attempted) and the number of variables selected at each split ( $m=10$ and $m=3$ attempted) resulted in no changes in overall accuracy greater than $1 \%$.

Probability estimates of class membership for each pixel were produced by the classifier, based on the proportion of class assignment votes across the 500 trees (Breiman, Cutler, Liaw, \& Wiener, 2012; Loosevelt et al. 2012). These probabilities were aggregated to the field level based on the mean of the pixels within a delineated field (similar to Section 3.3.6). However, in an attempt to reduce the influence of mixed pixels near field boundaries on the per-field probabilities, field polygons of an acceptable shape and size, were shrunk. Loosevelt et al. (2012), had compared uncertainty measures for boundary versus inner-field pixels, in a pixel-based Random Forest classification of synthetic aperture radar imagery, finding slightly higher degrees of uncertainty in boundary pixels. Wicks et al. (2002) had aggregated per-pixel characteristics to the field level using shrunken polygons to avoid mixed pixels. 
Perimeter area ratios are commonly used for quantifying polygon shape and compactness, and are also sensitive to shape area (Bogaert, Rousseau, Van Hecke, \& Impens, 2000; Remmel, Ko, \& Bello, 2010). Here, an area-to-perimeter ratio, simply the field area divided by the perimeter, was used to roughly distinguish more compact fields from less compact fields, as well as larger area fields from smaller fields. Using a negative buffer, field polygons, including alternate boundary representations (Section 3.4.2), were shrunk between 0 meters and 30 meters (increments of 5 meters) based on the area-to-perimeter ratio range into which they fell. The area-to-perimeter ratio intervals were determined by trial and error, attempting to remove as many of the nearboundary pixels as possible, while preserving the number of pixels considered for smaller or non-compact fields. The intervals are shown below, in Table 3.4.

Table 3.4 Polygon shrinking distance for area-to-perimeter ratio intervals.

\begin{tabular}{cc}
\hline Area/Perimeter $(\mathrm{m})$ & Shrinking distance $(\mathrm{m})$ \\
\hline$\geq 31$ & 30 \\
$\geq 28$ and $<31$ & 25 \\
$\geq 25$ and $<28$ & 20 \\
$\geq 22$ and $<25$ & 15 \\
$\geq 18$ and $<22$ & 10 \\
$\geq 15$ and $<18$ & 5 \\
$<15$ & 0 \\
\hline
\end{tabular}

Following the field polygon shrinking, per-pixel probabilities of class membership were aggregated to the area delineated by the shrunken polygons. These 
aggregated, per-field probability estimates were then assigned to the corresponding fullsized field polygons.

\subsubsection{Simulation}

Based on the probabilistic positional and thematic uncertainty representations, or PDFs, established in Sections 3.4.1, 3.4.2, and 3.4.3, 2000 realizations of LC structure and composition were generated. From each realization of each LC map, the three landscape metrics (P.AG, MFS, and SHDI) were recalculated. For each set of biodiversity measurements and each set of simulated landscape metrics, the linear candidate models were refit and model averaging performed.

\subsubsection{Realization generation and landscape metric calculation}

The first step in generating the LC map realizations was sensitizing the vertices of the field polygons, including those polygons representing alternate boundaries, with random positional error, based on the PDF representations of North-South and East-West positional uncertainty (Section 3.4.1). Due to the large number of vertices across the 1916 fields in the LCs (30,040 vertices, not including alternate boundary representations), vertex positions were simulated independently (i.e. spatially correlated error between vertices not considered). This was performed with Brown \& Heuvelink's (2007) Data Uncertainty Engine (version 3.1), wherein PRNs drawn form a uniform distribution were used to sample the CDFs representing the vertex position uncertainties (Section 2.5.1). The geometry of each sensitized field polygon (composed of sensitized vertex positions) was corrected for occasional cases of self intersections (ESRI, 2013b) caused by the 
spatially independent simulation of nearby vertices, followed by a recalculation of their area. The processing of the error-sensitized field boundaries was performed in ArcGIS Desktop 10.1 (ESRI, 2012). Next, all records within the GIS dataset, each representing an individual error-sensitized field polygon and containing information regarding:

(1) the boundary interpretation PDFs (for cases where there were alternate boundaries),

(2) the thematic uncertainty PDFs (for fields from year-2011 that had been classified), and

(3) the field area, updated to correspond with the error-sensitized vertices, were imported into 'the $\mathrm{R}$ environment for statistical computing' (The R Core Team, 2013) wherein the remainder of the steps for generating LC map realizations were carried out.

Each sensitized field polygon from the year-2011 dataset, including alternate boundaries, was then assigned a simulated thematic class. PRNs drawn form a uniform distribution were used to sample CDFs representing the per-field probabilities of class membership (Section 2.5.1; Section 3.4.3). Some very small fields that did not contain any of the TM pixels, and therefore had not been classified, were classed as 'Unknown' across all realizations (not considered in SHDI calculation). For the sensitized year-2012 fields, the ground referenced classes were used across all LC map realizations.

For each of the fields that had an alternate boundary representation, an individual representation was selected via sampling the CDFs representing the boundary interpretation uncertainty estimates, with PRNs drawn form a uniform distribution (Section 2.5.1; Section 3.4.2). 
The simulated field boundaries (vertex position and interpreted boundary) for both the year-2011 and year-2012 fields, as well as simulated crop classes for the year2011 fields, represented a full realization of the LC map dataset (both composition and configuration of these maps). For each of the LC maps in a full realization, the P.AG, MFS, and SHDI landscape metrics were recalculated. The MFS calculations omitted fields with and area of zero, indicating that they had been taken out of consideration in a given map realization.

Like the homogenous field-based simulation approach (Section 3.3), this approach to realization generation considered fields independently (independent PRNs) when assigning simulated thematic classes to fields, regardless of the potential for spatial autocorrelation between them. For the duration of this thesis, the approach to generating LC map realizations discussed here (Section 3.4.4.1) will be referred to as independent

\section{field simulation.}

\subsubsection{Alternate, grouped simulation approaches}

Since the independent field simulation approach (Section 3.4.4.1) did not consider potential spatial autocorrelation in crop type between fields, two alternate approaches to generating LC map realizations were explored. These alternate approaches considered vertex positional uncertainty and boundary interpretation uncertainty the same way as the independent field simulation approach, but differed in terms of how they considered thematic uncertainty. These approaches were aimed at roughly and simply considering spatial autocorrelation between fields within a LC. Similar to how the field-based simulation approach involved simulating pixels within a field with a common PRN, these 
approaches involved grouping fields within a LC that have the same most-likely class, and simulating grouped fields with a common PRN. Grouped fields with similar thematic class PDFs would be more likely to vary together in any given LC map realization.

The two alternate approaches considered will be referred to as the locally-grouped simulation approach and the landscape-grouped simulation approach. The locallygrouped simulation approach involved grouping adjacent fields (field boundaries within 20 meters of each other) of the same most-likely class. These adjacent fields were identified based on a series (one for each thematic class) of buffering, dissolving, and shrinking (negative buffer) operations performed in ArcGIS Desktop 10.2 (ESRI, 2013a). The landscape-grouped simulation approach involved simply grouping all fields of the same most-likely class within the same LC. While it may be a bit extreme to assume that all fields of the same most-likely class within an LC have spatially correlated error, the approach was offered as an alternate extreme to the completely independent approach of the independent field simulation.

A summary table of all simulation approaches used in this thesis, including those in Section 3.3.7, is provided below (Table 3.5). 
Table 3.5 Summary table of simulation approaches. All approaches to generating realizations of thematic class, that were used in this study, are summarized. For the independent field, locally-grouped, and landscape grouped simulation approaches, positional uncertainties were also considered, but separately.

\begin{tabular}{|c|c|c|c|c|c|c|}
\hline $\begin{array}{l}\text { Simulation } \\
\text { approach }\end{array}$ & $\begin{array}{l}\text { Description } \\
\text { location }\end{array}$ & $\begin{array}{l}\text { PDFs of class } \\
\text { membership }\end{array}$ & $\begin{array}{l}\text { PRNs for sampling } \\
\text { class membership } \\
\text { PDFs }\end{array}$ & $\begin{array}{c}\text { Spatial } \\
\text { autocorrelation } \\
\text { addressed } \\
\end{array}$ & Scale of use & Context of use \\
\hline $\begin{array}{l}\text { Independent } \\
\text { pixel-based }\end{array}$ & $\begin{array}{c}\text { Section } \\
3.3 .7\end{array}$ & Per-pixel & $\begin{array}{l}\text { Independent } \\
\text { between pixels }\end{array}$ & No & $\begin{array}{l}3 \mathrm{~km} \times 3 \mathrm{~km} \\
\text { landscapes }\end{array}$ & $\begin{array}{l}\text { Analysis of the effect of thematic } \\
\text { uncertainty, in the limited extent } \\
\text { classifications, on SHDI }\end{array}$ \\
\hline Field-based & $\begin{array}{c}\text { Section } \\
3.3 .7\end{array}$ & Per-pixel & $\begin{array}{l}\text { Common within } \\
\text { fields }\end{array}$ & Within-field & $\begin{array}{l}3 \mathrm{~km} \times 3 \mathrm{~km} \\
\text { landscapes }\end{array}$ & $\begin{array}{l}\text { Analysis of the effect of thematic } \\
\text { uncertainty, in the limited extent } \\
\text { classifications, on SHDI }\end{array}$ \\
\hline $\begin{array}{l}\text { Homogenous } \\
\text { field-based }\end{array}$ & $\begin{array}{c}\text { Section } \\
3.3 .7\end{array}$ & Per-field & $\begin{array}{l}\text { Common within } \\
\text { fields }\end{array}$ & Within-field & $\begin{array}{l}3 \mathrm{~km} \times 3 \mathrm{~km} \\
\text { landscapes }\end{array}$ & $\begin{array}{l}\text { Analysis of the effect of thematic } \\
\text { uncertainty, in the limited extent } \\
\text { classifications, on SHDI }\end{array}$ \\
\hline $\begin{array}{l}\text { Independent } \\
\text { field }\end{array}$ & $\begin{array}{l}\text { Section } \\
3.4 .4 .1\end{array}$ & Per-field & $\begin{array}{l}\text { Common within } \\
\text { fields }\end{array}$ & Within-field & $\begin{array}{c}1 \mathrm{~km} \times 1 \mathrm{~km} \\
\text { LCs }\end{array}$ & $\begin{array}{l}\text { Analysis of the effect of } \\
\text { thematic uncertainty, in the LC } \\
\text { maps, on SHDI coefficients }\end{array}$ \\
\hline $\begin{array}{l}\text { Locally- } \\
\text { grouped }\end{array}$ & $\begin{array}{l}\text { Section } \\
3.4 .4 .2\end{array}$ & Per-field & $\begin{array}{l}\text { Common for } \\
\text { nearby, *similar } \\
\text { fields within a LC }\end{array}$ & $\begin{array}{l}\text { Within-field \& } \\
\text { between-field }\end{array}$ & $\begin{array}{c}1 \mathrm{~km} \times 1 \mathrm{~km} \\
\text { LCs }\end{array}$ & $\begin{array}{l}\text { Analysis of the effect of } \\
\text { thematic uncertainty, in the LC } \\
\text { maps, on SHDI coefficients }\end{array}$ \\
\hline $\begin{array}{l}\text { Landscape- } \\
\text { grouped }\end{array}$ & $\begin{array}{l}\text { Section } \\
3.4 .4 .2\end{array}$ & Per-field & $\begin{array}{l}\text { Common for all } \\
\text { *similar fields within } \\
\text { a LC }\end{array}$ & $\begin{array}{l}\text { Within-field \& } \\
\text { between-field }\end{array}$ & $\begin{array}{c}1 \mathrm{~km} \times 1 \mathrm{~km} \\
\text { LCs }\end{array}$ & $\begin{array}{l}\text { Analysis of the effect of } \\
\text { thematic uncertainty, in the LC } \\
\text { maps, on SHDI coefficients }\end{array}$ \\
\hline
\end{tabular}

\footnotetext{
* 'similar fields', in this case, refers to fields that have the same hard class.
} 


\subsubsection{Extension of simulation through model averaging}

For each of the 2000 landscape metric set realizations (generated under any of the three simulation approaches outlined in Sections 3.4.4.1 and 3.4.4.2), and for each set of biodiversity measurements, the seven candidate models were refit (see Section 2.6.1 for the candidate model details). AICc model averaging was performed on each set of refit candidate models, each time storing the averaged coefficients and standard error estimates. After processing all landscape metric realizations, the following values were calculated for each biodiversity model (see Burnham \& Anderson, 2002; Anderson, 2008):

$\widehat{\bar{\theta}}_{\text {hard }} \quad$, representing the model averaged coefficient estimates (for either P.AG, MFS, or SHDI) based on the hard dataset (reference field boundaries, with year-2011 crop types based on hardened image classification);

$\widehat{\mathbf{s e}}\left(\widehat{\bar{\Theta}}_{\text {hard }}\right)$, representing the unconditional model averaging standard error estimates associated with $\widehat{\bar{\theta}}_{\text {hard }}$;

$\widehat{\bar{\theta}}_{\text {sim }} \quad$, representing the simulated model averaged coefficient estimates (for either P.AG, MFS, or SHDI), averaged across all realizations;

$\widehat{\mathbf{s e}}\left(\widehat{\bar{\Theta}}_{\text {sim }}\right)$, representing the unconditional model averaging standard error estimates associated with $\widehat{\bar{\theta}}_{\text {sim }}$, pooled across all realizations (converted to variance and averaged); and

$\boldsymbol{\sigma}_{\text {sim }} \quad$, representing the standard deviation in $\widehat{\bar{\Theta}}_{\text {sim }}$, across all realizations. 
$\widehat{\operatorname{se}}(\hat{\bar{\theta}})$ is the square root of $\widehat{\operatorname{var}}(\widehat{\hat{\theta}})$, where $\widehat{\operatorname{var}}(\widehat{\hat{\theta}})$ is the unconditional variance estimate (see Anderson, 2008, p. 111) which considers variance in the sample, given the model, and the variance associated with model selection (Section 2.6.1).

An illustrated example of these terms is shown in Figure 3.17, below.

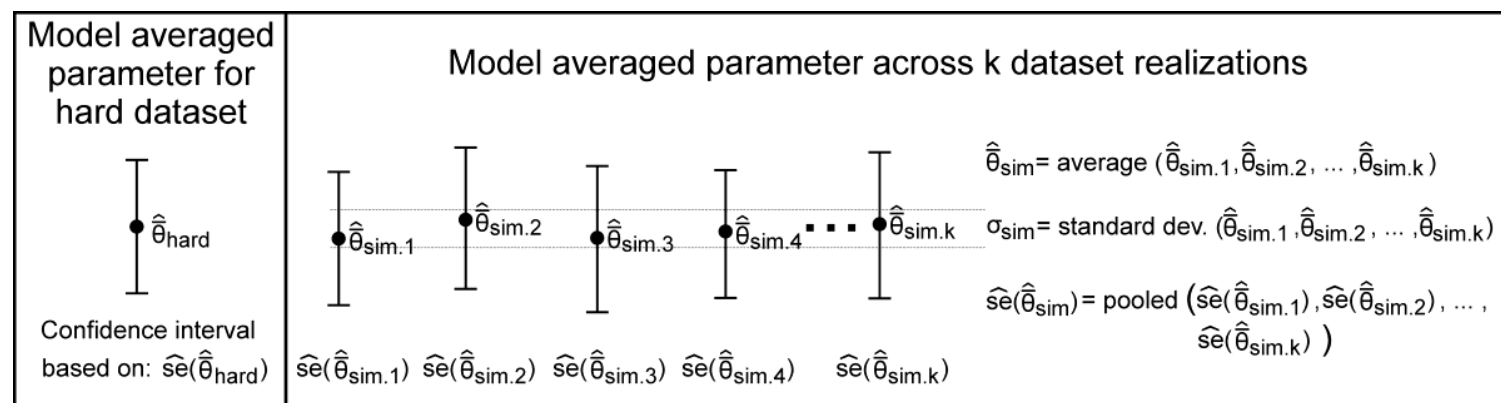

Note: $\widehat{\operatorname{se}}(\hat{\bar{\theta}})=\sqrt{\operatorname{var}(\hat{\bar{\theta}})}$, where $\operatorname{var}(\hat{\bar{\theta}})$ is the unconditional variance estimate (see Anderson, 2008, p.111)

Figure 3.17 An illustrated example of the values retained and calculated from model averaging of the hard dataset and dataset realizations.

After model averaging was completed for all 2000 realizations of the landscape metric set and corresponding, refit candidate model sets, the mean coefficients and standard error estimates for each biodiversity model were calculated and checked for convergence (via cumulative variance plots, like in Section 3.3.7). 


\section{Results}

Results of the SHDI simulation comparison (Sections 3.3 and 4.1) and the biodiversity model uncertainty analysis (Sections 3.4 and 4.2) are presented in this section. These include the soft and hardened (most-likely class assigned) classification results with accuracy assessments, the distributions of simulated landscape metrics, the distributions of simulated model coefficients, and the convergence checks.

\subsection{SHDI simulation comparison results}

The results of the limited extent classification and SHDI simulation comparison (Section 3.3) are presented in this section, aimed at addressing research question 1 (Section 1.3). The results of the pixel-based soft classification (posterior probabilities) are provided (Section 4.1.1) and characterized with $E_{\mathrm{n}}$ measures (Section 4.1.2). The accuracies of the hardened classification are then briefly compared through traditional, overall accuracy measures (Section 4.1.3). Examples of map realizations are then provided, followed by characteristics of the simulated SHDI distributions (Sections 4.1.4 and 4.1.5).

\subsubsection{Probabilities of class membership (soft classification)}

Figures 4.1, 4.2, and 4.3, below, show the posterior probabilities of class membership (all classes, except for Abandoned), determined by the Bayesian classifier (Section 3.3.6), for the single date TM, multi-date TM, and QB image sets respectively. 
Outlines of the trimmed field boundaries (Figure 3.5), showing ground referenced crop types, are also included in these figures. 


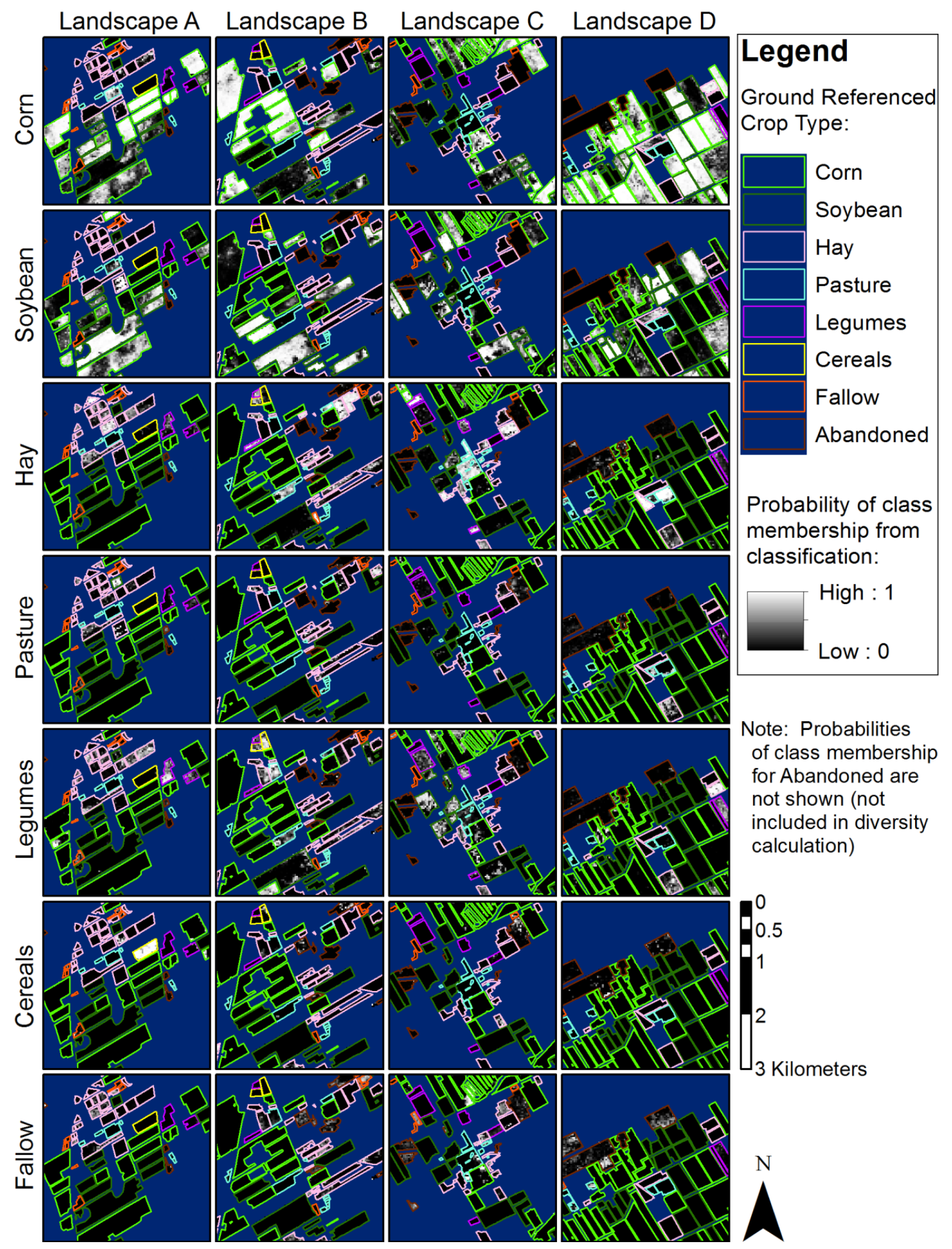

Figure 4.1 Posterior probabilities of class membership from a Bayesian

classification of the 19 June TM image set. 


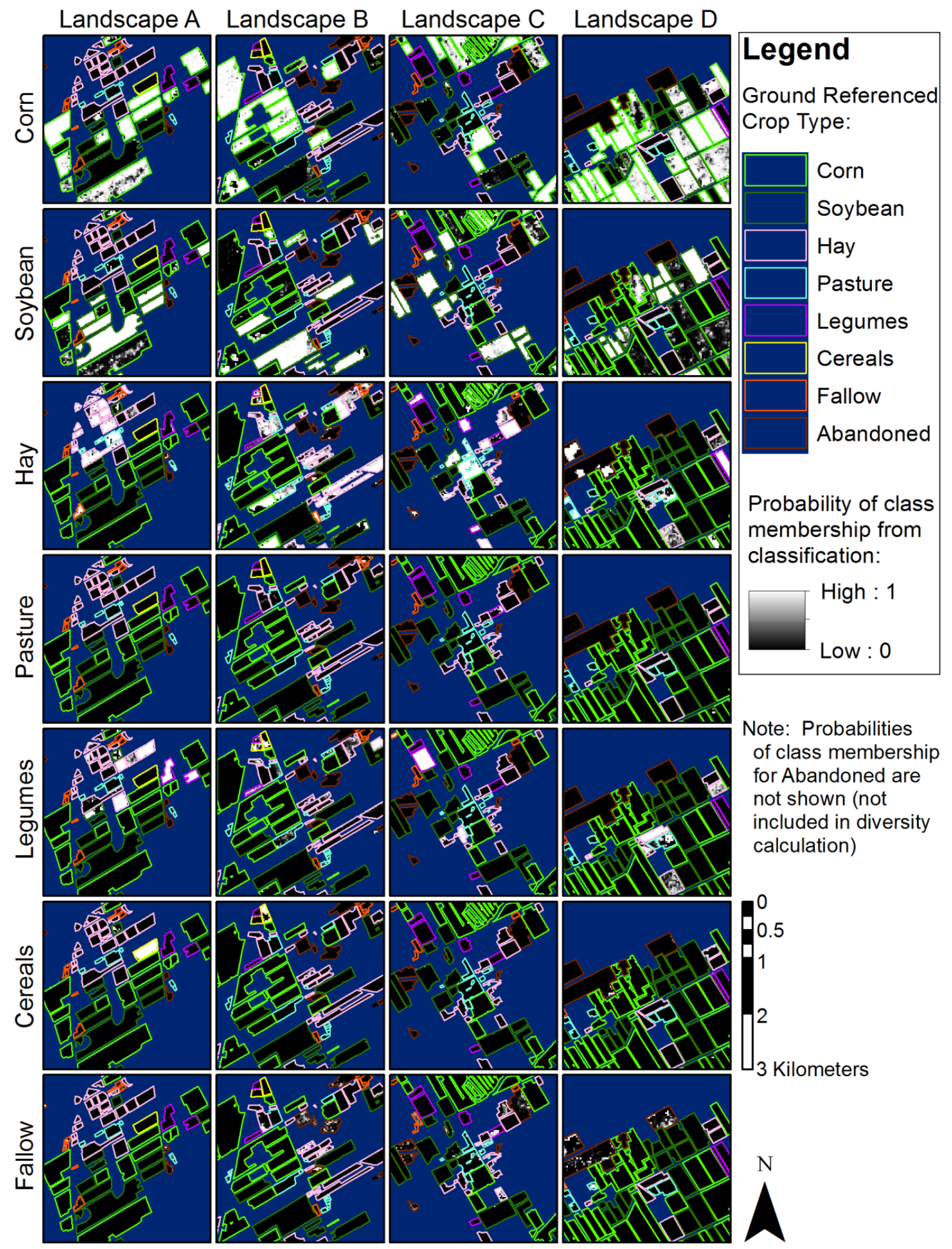

Figure 4.2 Posterior probabilities of class membership from a Bayesian classification of the multi-date TM image set. 


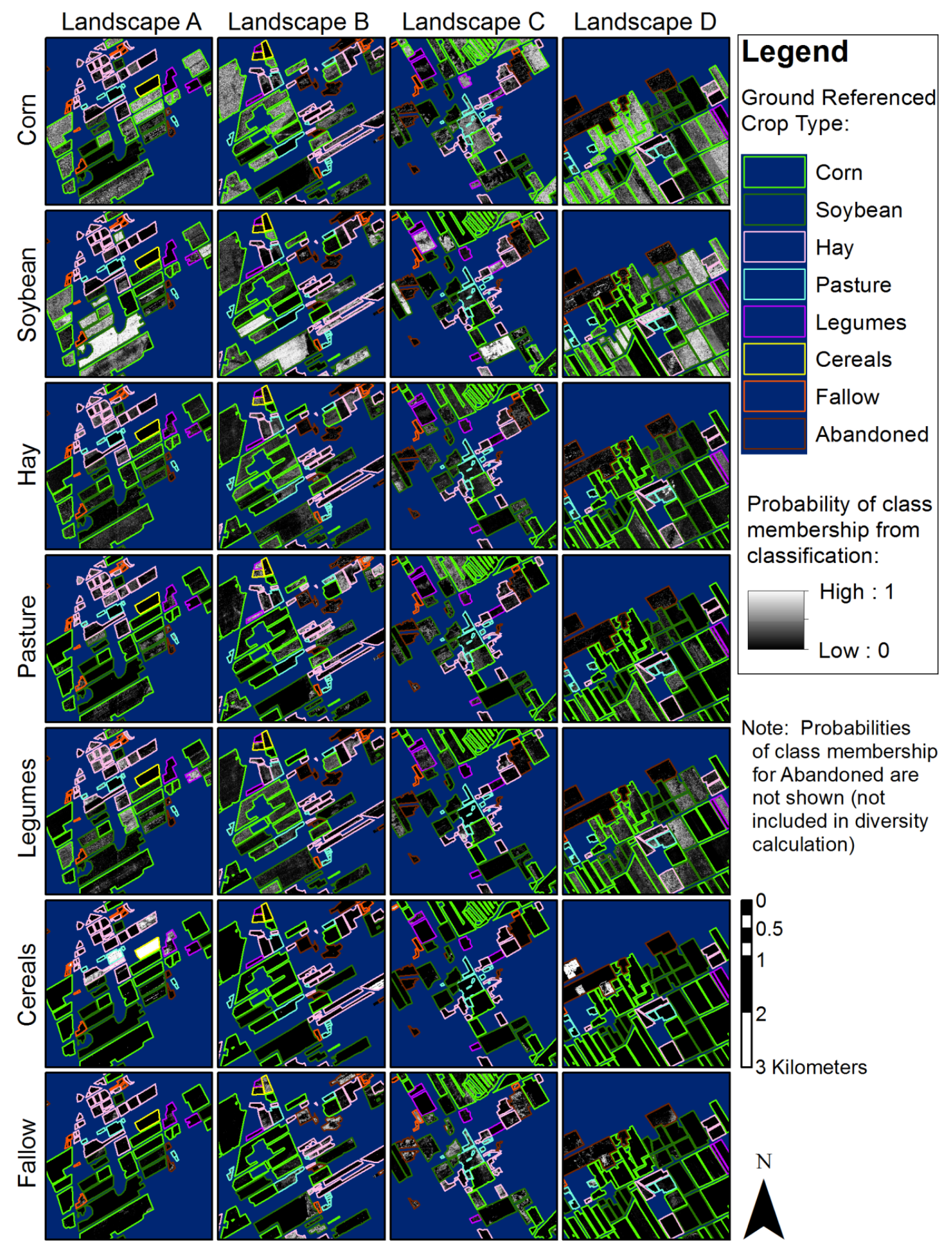

Figure 4.3 Posterior probabilities of class membership from a Bayesian classification of the 29 August QB image set. 
Per-pixel probabilities of class membership from the TM classifications (Figs. 4.1 and 4.2) were quite consistent within fields, relative to those for QB classification (Fig. 4.3), wherein these probabilities appeared very speckled. The TM classifications had probabilities that were high or low, often with a clear majority in favor of a particular class within the field boundaries. In the QB classification, on the other hand, there was a high degree of class confusion within the boundaries of many fields, with probabilities seldom showing a clear majority. In many of the cases where there was a clear class majority, it was often in the wrong class. Examples include Pasture (Landscape A) or Abandoned (Landscape D) fields being assigned very confidently to Cereals, or Legumes fields (Landscape C) being assigned confidently to Soybean (Fig. 4.3). These issues suggest the at QB classification was of very poor quality.

While the TM classifications were better in terms of consistency, there were still several cases of fields showing high degrees of certainty towards incorrect classes. There were several of these cases for the Hay, Pasture, Legumes, and Fallow classes (these will be referred to as the grass-like classes, as they often contained similar vegetation types, particularly grasses). Hay, Pasture, and Legumes had shown low separabilities in the training data, as well as Fallow and Abandoned (Section 3.3.6). There was confusion between Corn and Soybean (e.g. Landscape D), which had also shown low separabilities. These cases of very low probabilities in the correct class, and very high probabilities in an incorrect class suggest that there were issues in obtaining a training set that was fully representative of all crop conditions (e.g. growth stage, crop health, crop variant, etc.) from within the limited extent (expanded on in the Discussion, Section 5.1.1.2). 
Pasture, which had very limited training samples (Fig. 3.7), had poor representation across all image classifications.

\subsubsection{Normalized entropy}

Table 4.1, below, shows the mean $E_{\mathrm{n}}$ for the per-pixel and per-field probabilities of class membership (Fig. 3.9), across the four landscapes and three image set classifications. On average, for both the per-pixel and per-field representations, the probabilities exhibited higher entropies (hard classes assigned with higher ambiguity; Section 2.4.1) for the QB classifications, followed by the single-date TM classifications, and then the multi-date TM classifications.

Table 4.1 Mean normalized entropy values for the four landscapes and three image set classifications.

\begin{tabular}{|c|c|c|c|c|c|}
\hline \multirow[b]{2}{*}{ Representation } & \multirow{2}{*}{$\begin{array}{l}\text { Image set } \\
\text { classification }\end{array}$} & \multicolumn{4}{|c|}{ Mean normalized entropy } \\
\hline & & $\begin{array}{c}\text { Landscape } \\
\text { A } \\
\end{array}$ & $\begin{array}{c}\text { Landscape } \\
\text { B } \\
\end{array}$ & $\begin{array}{c}\text { Landscape } \\
\text { C } \\
\end{array}$ & $\begin{array}{c}\text { Landscape } \\
\text { D } \\
\end{array}$ \\
\hline \multirow{3}{*}{ Per-pixel } & $\begin{array}{c}\text { Single date } \\
\text { TM }\end{array}$ & 0.203 & 0.189 & 0.241 & 0.165 \\
\hline & $\begin{array}{l}\text { Multi-date } \\
\text { TM }\end{array}$ & 0.051 & 0.067 & 0.061 & 0.071 \\
\hline & QB & 0.417 & 0.491 & 0.494 & 0.466 \\
\hline \multirow{3}{*}{ Per-field } & $\begin{array}{c}\text { Single date } \\
\text { TM }\end{array}$ & 0.340 & 0.356 & 0.440 & 0.251 \\
\hline & $\begin{array}{l}\text { Multi-date } \\
\text { TM }\end{array}$ & 0.163 & 0.223 & 0.208 & 0.174 \\
\hline & QB & 0.577 & 0.670 & 0.703 & 0.608 \\
\hline
\end{tabular}


Table 4.2 , below, shows mean $E_{\mathrm{n}}$ by class, across the per-field classifications of the three image sets. On average, $E_{\mathrm{n}}$ values were higher for the grass-like classes and for Abandoned, all of which tended to have lower separabilities (Section 3.3.6). Legumes fields in the multi-date TM classifications were an exception to this, showing the lowest overall entropy. Corn, Soybean, and Cereals generally had lower entropy, particularly in the multi-date TM classifications.

Table 4.2 Mean normalized entropy values by class, across the per-field classifications of the three image sets.

\begin{tabular}{ccccccccc}
\hline \multirow{2}{*}{$\begin{array}{c}\text { Image set } \\
\text { classification }\end{array}$} & \multicolumn{7}{c}{ Mean normalized entropy, by class } \\
\cline { 2 - 8 } & Corn & Soybean & Hay & Pasture & Legumes & Cereals & Fallow & Abandoned \\
\hline $\begin{array}{c}\text { Single date } \\
\text { TM }\end{array}$ & 0.259 & 0.267 & 0.511 & 0.427 & 0.490 & 0.243 & 0.530 & 0.557 \\
$\begin{array}{c}\text { Multi-date } \\
\text { TM }\end{array}$ & 0.173 & 0.150 & 0.225 & 0.277 & 0.111 & 0.185 & 0.320 & 0.346 \\
QB & 0.659 & 0.551 & 0.701 & 0.724 & 0.619 & 0.293 & 0.680 & 0.702 \\
\hline
\end{tabular}

Additional information related to the differences in $E_{\mathrm{n}}$ between correctly and incorrectly classified fields is available in Appendix F.

\subsubsection{Accuracy of the hardened classifications}

The error matrices for the hardened per-pixel and per-field classifications of all three image sets can be seen in Appendix F. Table 4.3, below, shows the overall accuracies of each of these hardened classifications, both overall and across all four landscapes individually. 
Table 4.3 Overall accuracy and landscape-specific accuracy of the per-pixel and per-field classifications.

\begin{tabular}{|c|c|c|c|c|c|c|}
\hline \multirow{2}{*}{$\begin{array}{l}\text { Image } \\
\text { set }\end{array}$} & \multirow{2}{*}{$\begin{array}{l}\text { Pixel vs. field } \\
\text { representation }\end{array}$} & \multirow{2}{*}{$\begin{array}{c}\text { Overall } \\
\text { accuracy } \\
(\%)\end{array}$} & \multicolumn{4}{|c|}{ Overall accuracy by landscape (\%) } \\
\hline & & & $\begin{array}{c}\text { Landscape } \\
\text { A }\end{array}$ & $\begin{array}{c}\text { Landscape } \\
\text { B } \\
\end{array}$ & $\begin{array}{c}\text { Landscape } \\
\text { C } \\
\end{array}$ & $\begin{array}{c}\text { Landscape } \\
\text { D } \\
\end{array}$ \\
\hline \multirow{2}{*}{$\begin{array}{c}\text { Single } \\
\text { date TM }\end{array}$} & Pixel & 59.3 & 57.1 & 62.8 & 50.9 & 63.1 \\
\hline & Field & 65.1 & 60.3 & 71.9 & 55.9 & 68.9 \\
\hline \multirow{2}{*}{$\begin{array}{l}\text { Multi- } \\
\text { date TM }\end{array}$} & Pixel & 72.0 & 78.8 & 74.4 & 70.5 & 66.6 \\
\hline & Field & 77.6 & 84.0 & 83.0 & 78.6 & 68.8 \\
\hline \multirow{2}{*}{ QB } & Pixel & 48.9 & 49.4 & 43.3 & 35.4 & 61.2 \\
\hline & Field & 63.2 & 53.7 & 65.9 & 45.0 & 78.8 \\
\hline
\end{tabular}

Overall accuracies ranged from very low (e.g. QB classifications) to moderate (e.g. multi-date TM classifications of some landscapes). The per-pixel QB classifications, in which the probabilities had a very speckled appearance (Fig. 4.3) were the lowest overall, at $48.9 \%$. The aggregation to per-field representation improved the accuracy to $63.2 \%$, still very poor. Overall accuracies for the single date TM classifications were also low, though slightly better than the QB classifications. The multi-date TM classifications had the highest overall accuracies, with a moderate $72.0 \%$ for the per-pixel classification, and $77.6 \%$ for the per-field classification. This accuracy was somewhat lower than the $82 \%$ accuracy achieved for the province of Ontario by the AAFC Annual Crop Inventory, though there were differences in terms of classification scheme and imagery (AAFC, n.d.).

Again, it should be noted that the objective was not to have the most accurate classifications possible, but to have a range of accuracies and classification ambiguity 
available for comparison. Overall classification accuracies could have been as high as $85.9 \%$ to $92.9 \%$ across the landscapes, had some classes been merged (Appendix F, Tables E.26 to E.29). There were also several other ways that these accuracies could have been improved (discussed in Section 5.1.1).

\subsubsection{Landscape realizations}

Figures 4.4, 4.5, and 4.6, below, show the hardened classifications and three realizations of each simulation approach for the single date TM, multi-date TM, and QB classifications respectively. Only Landscape A is shown, which contained every considered crop type. 
Independent pixel-based
simulation
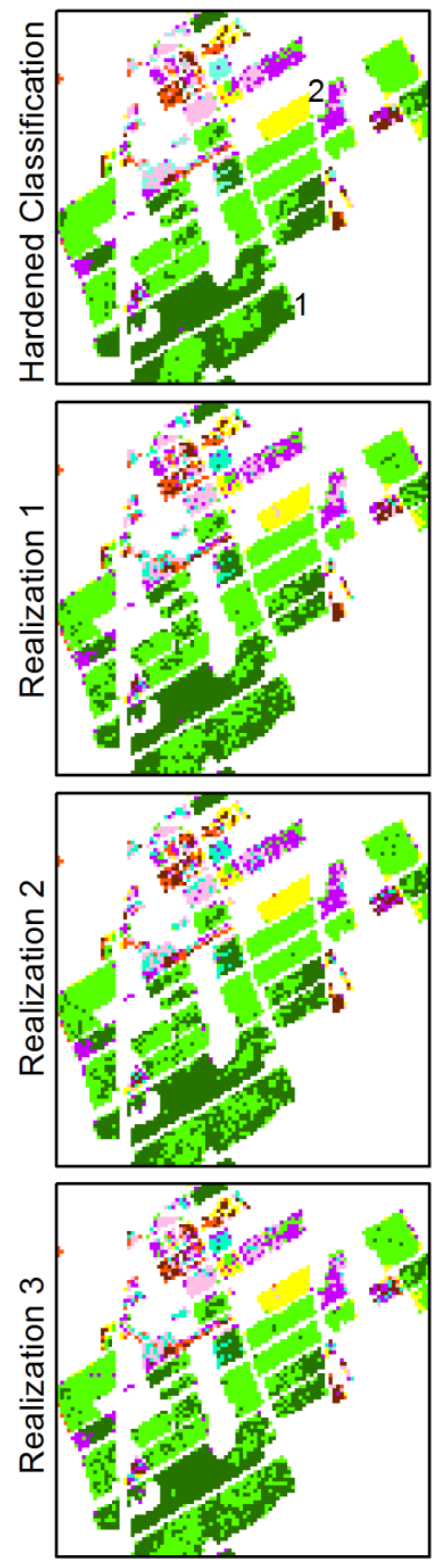

Field-based simulation
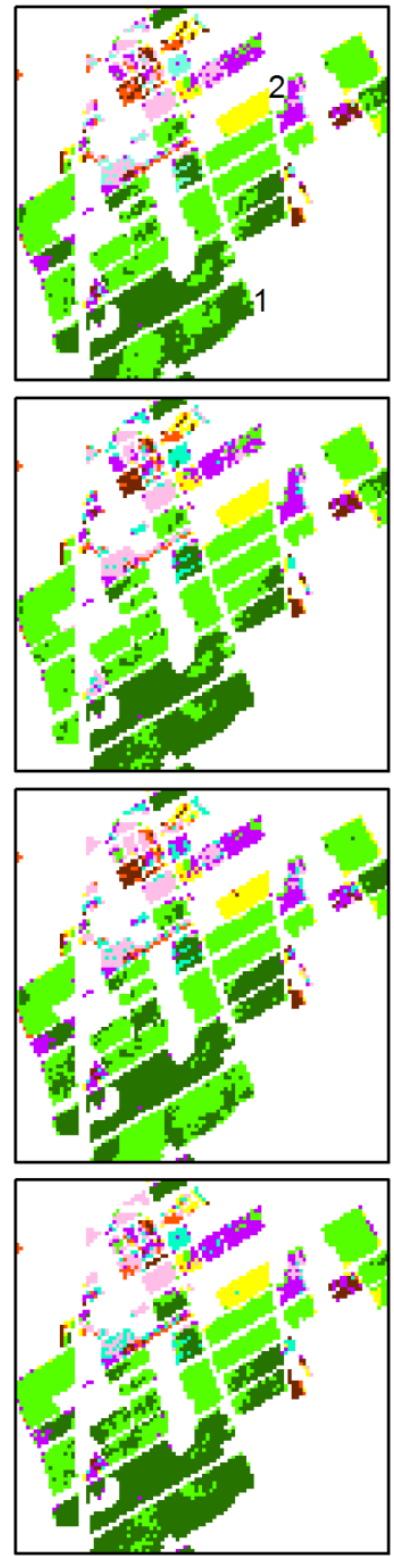

Homogenous field-based simulation
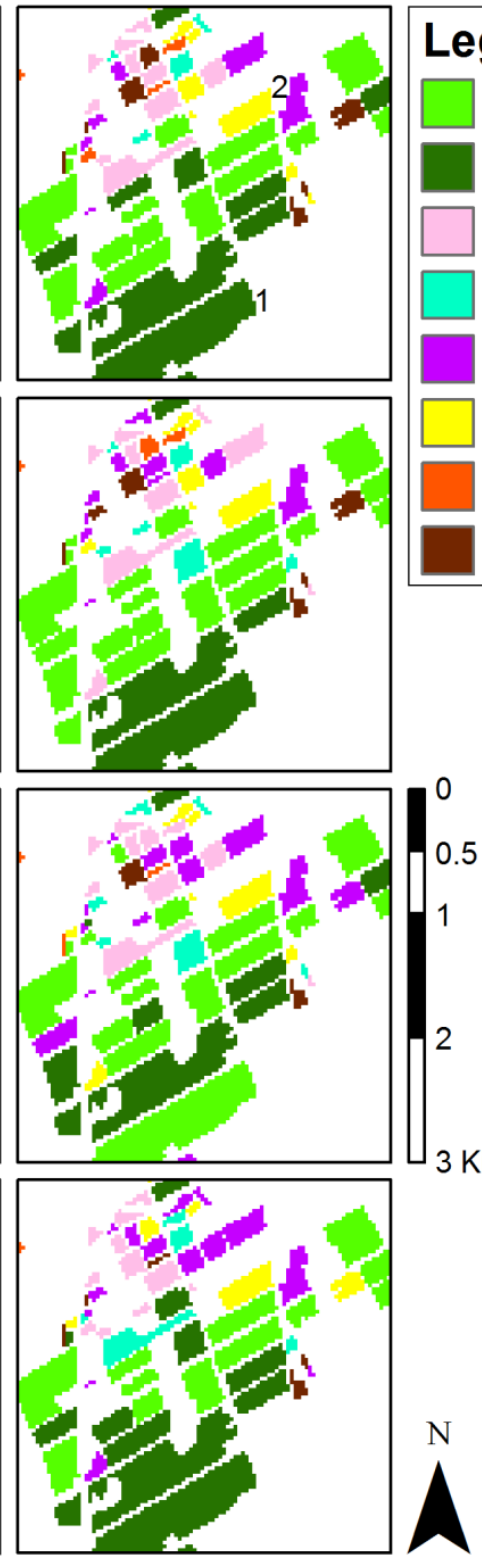

Figure 4.4 Hardened classifications and examples of realizations of Landscape

A, based on the single date TM classifications. 


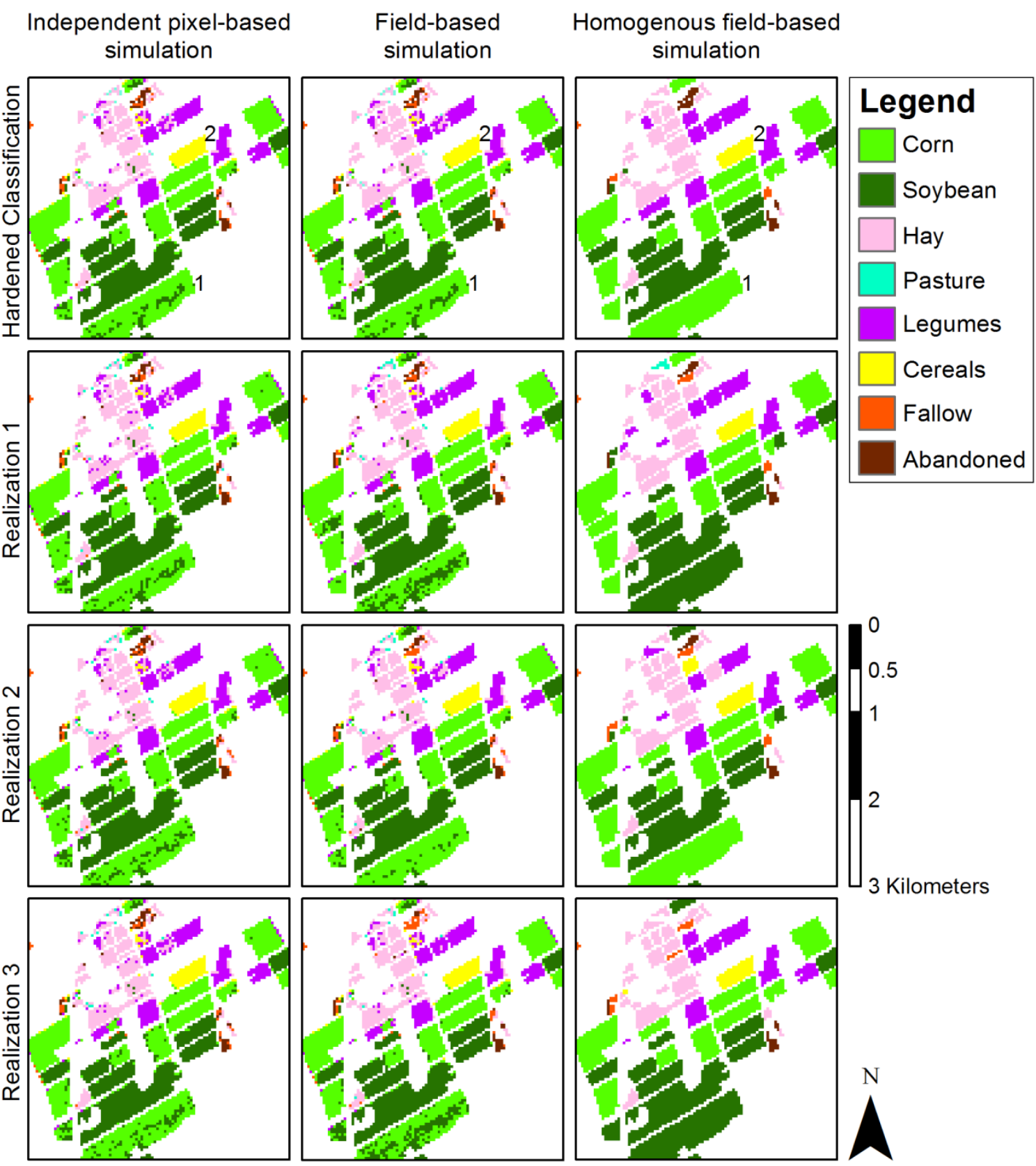

Figure 4.5 Hardened classifications and examples of realizations of Landscape A, based on the multi-date TM classifications. 


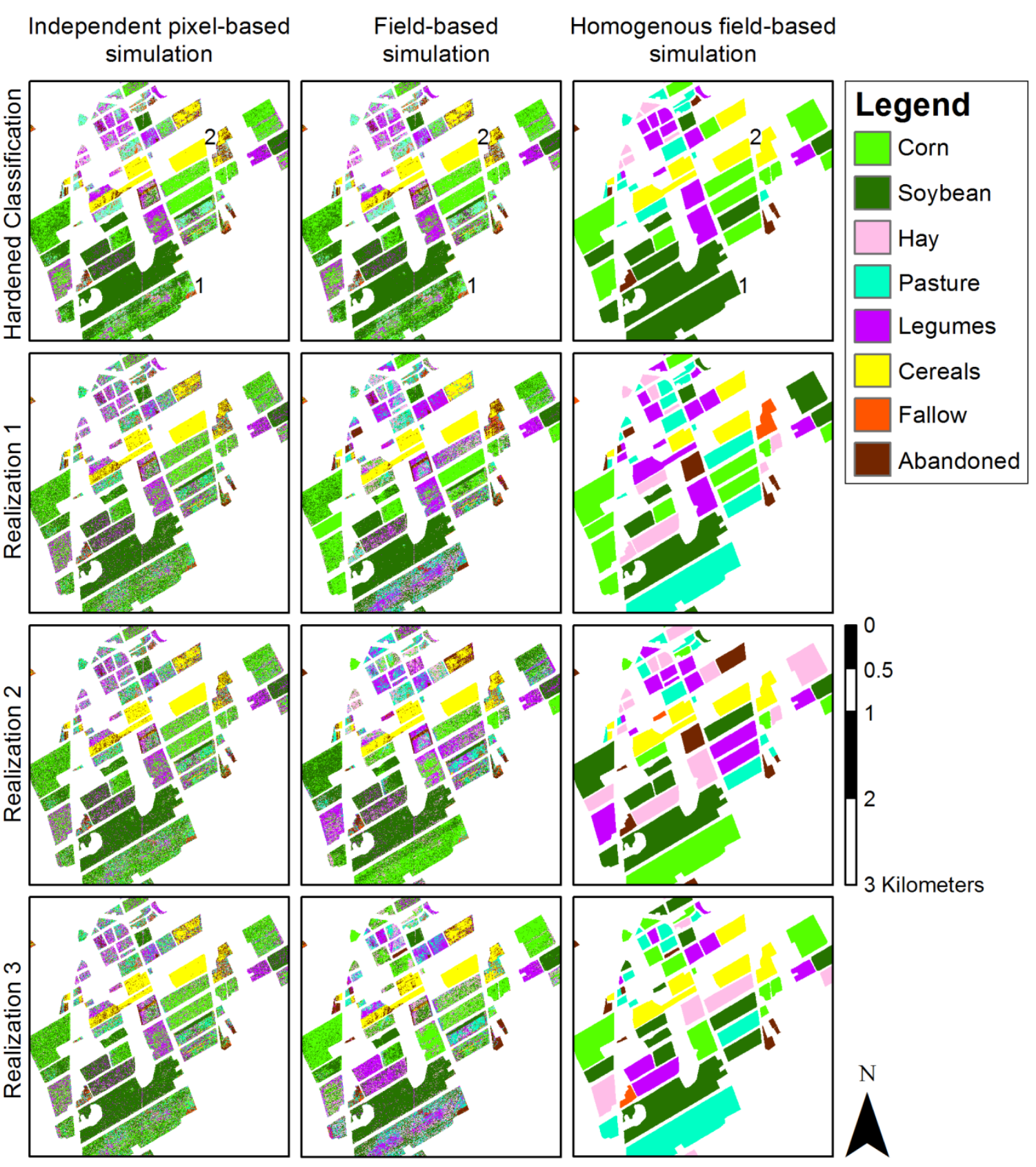

Figure 4.6 Hardened classifications and examples of realizations of Landscape A, based on the QB classifications. 
There were some clear differences between simulation approaches within Landscape A. The large, southernmost Corn field in the landscape (marked as ' 1 ' in the Hardened Classification panels) provides clear examples of these differences. The field, which had a moderate amount of ambiguity between Corn and Soybean, had a speckling of these classes across the independent pixel-based realizations of the single date TM classification (Fig. 4.4), with smaller changes of within-field class proportions across the realizations. For the field-based realizations, there are larger changes of within-field class proportions across the three realizations, with much less speckle.

The difference between the independent pixel-based simulation and the fieldbased simulation for the southernmost Corn field (marked as '1') was less evident in the multi-date TM classification (Fig. 4.5), where entropy was generally lower, relative to the single date TM classification (Table 4.2). Shifts between Corn and Soybean proportions within the field appeared only slightly larger for the field-based realizations than for the independent pixel-based realizations, relative to those shifts seen in the single date TM classifications (Fig. 4.4).

Independent pixel-based simulation and the field-based simulation approaches again differed for the QB classifications (Fig. 4.6). The southernmost Corn field was much more speckled, for both simulation approaches, than in the single and multi-date TM classifications. The independent pixel-based realizations showed minor shifts in class proportions within the field, though larger shifts could be seen in the field-based realizations, particularly Realization 2 (relative to Realizations 1 and 3).

For all image set classifications (Figs. 4.4, 4.5, 4.6), there was a clear difference between homogenous field-based approach and the other simulation approaches, when 
looking at the southernmost Corn field (marked '1'). A complete lack of within-field variability resulted in large shifts in class proportion from realization to realization.

While the differences between the simulation approaches were quite clear when looking at the southernmost Corn field, there were fields for which these differences were not as apparent. For example, the Cereals field that appeared in all classifications (marked as '2'), shows little difference in terms of speckle or shifts in class proportions across all three realizations of all simulation approaches. In all classifications this field had been classified with very high confidence relative to the southernmost Corn field.

\subsubsection{Simulated SHDI distributions}

Figure 4.7, below, shows the distributions of simulated SHDI values across the four landscapes, three image set classifications, and three simulation approaches. Clear differences in variance between the three simulation approaches can be seen. Offset between the simulated SHDI distributions and the reference SHDI values differed between image sets. Also, for all simulated SHDI distributions, an offset can be seen between the distribution median and the SHDI value of the hardened classification. These differences will be explored further, below, starting with Table 4.4, which lists the variance for each distribution shown in Figure 4.7. 

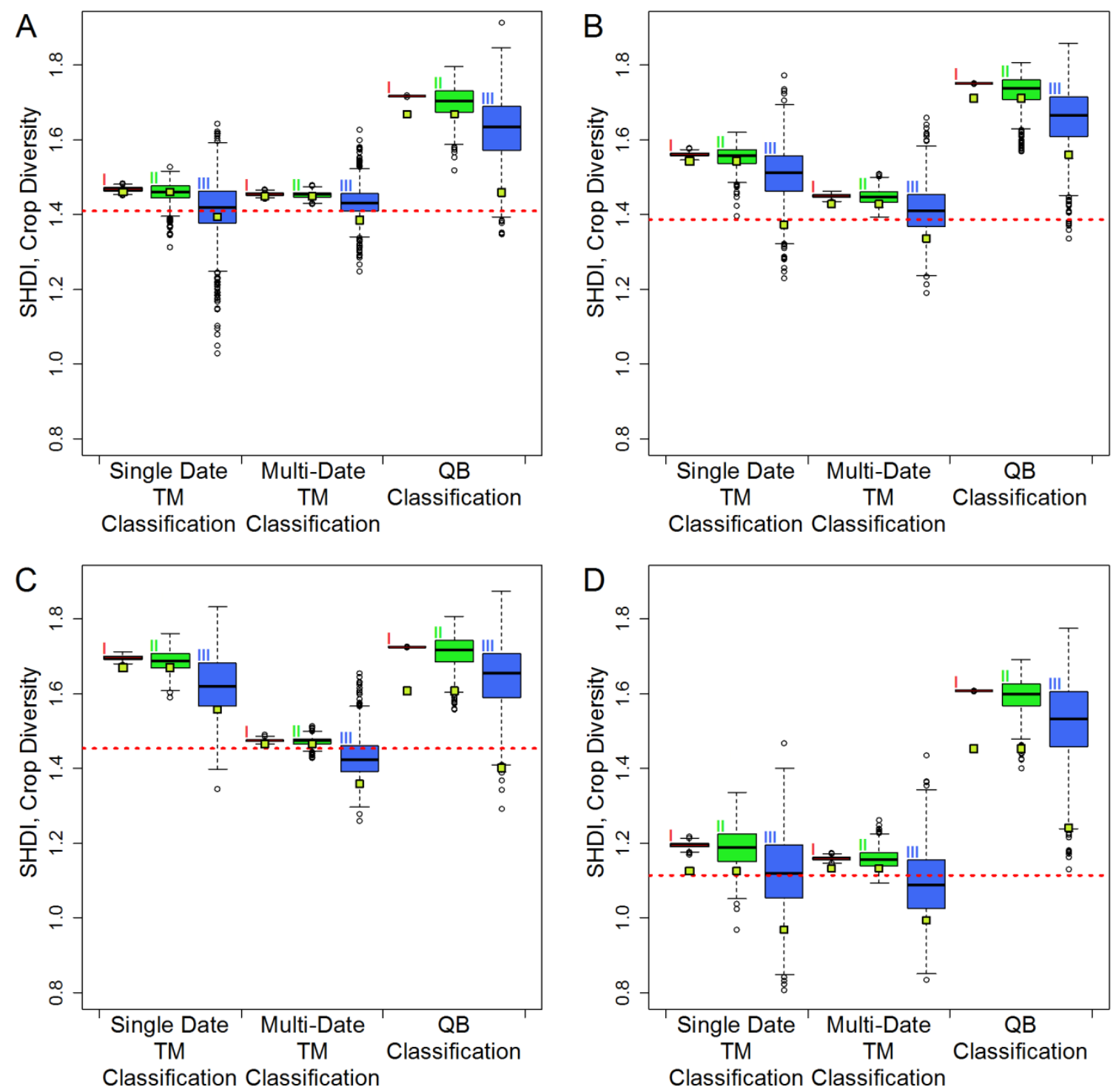

\begin{tabular}{|c|c|}
\hline $\begin{array}{l}\text { Legend } \\
\qquad \text { Independent pixel-based simulation (I) }\end{array}$ & $\begin{array}{l}\text { - Crop diversity of ground } \\
\text { referenced landscape map }\end{array}$ \\
\hline Field-based simulation (||) & $\square$ Crop diversity of hardened \\
\hline Homogenous field-based simulation (III) & classification \\
\hline
\end{tabular}

Figure 4.7 Simulated SHDI distributions across four landscapes, three image set classifications, and three simulation types. Distributions are shown for (A) Landscape A, (B) Landscape B, (C) Landscape C, and (D) Landscape D. The reference SHDI value (red dashed line) is not the original reference metric, but the SHDI of the fully ground referenced, thematically exhaustive, trimmed maps (Sections 3.3.2 and 3.3.3). 
Table 4.4 Variance of simulated SHDI distributions by image set classification, simulation approach, and landscape.

\begin{tabular}{|c|c|c|c|c|c|}
\hline \multirow{2}{*}{$\begin{array}{l}\text { Image set } \\
\text { classification }\end{array}$} & \multirow{2}{*}{$\begin{array}{l}\text { Simulation } \\
\text { approach }\end{array}$} & \multicolumn{4}{|c|}{ Simulated SHDI variance by landscape } \\
\hline & & $\begin{array}{c}\text { Landscape } \\
\text { A }\end{array}$ & $\begin{array}{c}\text { Landscape } \\
\text { B }\end{array}$ & $\begin{array}{c}\text { Landscape } \\
\text { C }\end{array}$ & $\begin{array}{c}\text { Landscape } \\
\text { D }\end{array}$ \\
\hline \multirow{3}{*}{$\begin{array}{c}\text { Single date } \\
\text { TM } \\
\text { classification }\end{array}$} & $\begin{array}{l}\text { Independent } \\
\text { pixel-based }\end{array}$ & $2.85 \times 10^{-5}$ & $2.87 \times 10^{-5}$ & $3.68 \times 10^{-5}$ & $4.97 \times 10^{-5}$ \\
\hline & Field-based & $6.89 \times 10^{-4}$ & $7.44 \times 10^{-4}$ & $8.05 \times 10^{-4}$ & $2.94 \times 10^{-3}$ \\
\hline & $\begin{array}{l}\text { Homogenous } \\
\text { field-based }\end{array}$ & $6.30 \times 10^{-3}$ & $5.41 \times 10^{-3}$ & $6.35 \times 10^{-3}$ & $1.10 \times 10^{-2}$ \\
\hline \multirow{3}{*}{$\begin{array}{c}\text { Multi-date } \\
\text { TM } \\
\text { classification }\end{array}$} & $\begin{array}{l}\text { Independent } \\
\text { pixel-based }\end{array}$ & $1.47 \times 10^{-5}$ & $2.43 \times 10^{-5}$ & $1.55 \times 10^{-5}$ & $2.22 \times 10^{-5}$ \\
\hline & Field-based & $6.55 \times 10^{-5}$ & $3.84 \times 10^{-4}$ & $1.29 \times 10^{-4}$ & $7.08 \times 10^{-4}$ \\
\hline & $\begin{array}{l}\text { Homogenous } \\
\text { field-based }\end{array}$ & $1.88 \times 10^{-3}$ & $4.41 \times 10^{-3}$ & $3.17 \times 10^{-3}$ & $8.12 \times 10^{-3}$ \\
\hline \multirow{3}{*}{$\begin{array}{c}\text { QB } \\
\text { classification }\end{array}$} & $\begin{array}{l}\text { Independent } \\
\text { pixel-based }\end{array}$ & $3.15 \times 10^{-7}$ & $1.28 \times 10^{-7}$ & $3.02 \times 10^{-7}$ & $3.11 \times 10^{-7}$ \\
\hline & Field-based & $1.73 \times 10^{-3}$ & $1.60 \times 10^{-3}$ & $1.73 \times 10^{-3}$ & $2.07 \times 10^{-3}$ \\
\hline & $\begin{array}{l}\text { Homogenous } \\
\text { field-based }\end{array}$ & $8.06 \times 10^{-3}$ & $7.41 \times 10^{-3}$ & $7.57 \times 10^{-3}$ & 0.0118 \\
\hline
\end{tabular}

For all four landscapes in each image set, the variance of the independent pixelbased SHDI distribution was less than that of the corresponding field-based SHDI distribution. In turn, the variance of each field-based SHDI distribution was less that of the corresponding homogenous field-based SHDI distribution.

Looking at corresponding variances of the same image set and landscape, on average, the proportion of variance associated with independent pixel-based simulation relative to that of field-based simulation was $4.9 \%$ (standard deviation: $6.5 \%$ ). Breaking 
this ratio down by image set (Table 4.4; visible in Fig. 4.7), the average ratio was highest for the multi-date TM classifications (at 11.0\%), which tended to have the lower perpixel mean $E_{\mathrm{n}}$ values (Table 4.1). The average ratio was lowest for the QB classifications (at $1.47 \times 10^{-4} \%$ ) which had the highest per-pixel mean $E_{\mathrm{n}}$ values (Table 4.1 ). This suggests that, in terms of variance, the effect of using the field-based approach versus the independent pixel-based approach (both used per-pixel probabilities of class membership; Section 3.3.7) was larger for those classifications that had higher per-pixel mean $E_{\mathrm{n}}$ values.

Again looking at the corresponding variances in the same image set and landscape, on average, the proportion of variance associated with field-based simulation relative to that of homogenous field-based simulation was $14.4 \%$ (standard deviation: 7.6\%). Breaking this proportion down by image set (Table 4.4; visible in Fig. 4.7), the average proportion was, this time, lowest for the multi-date TM classifications (at $6.2 \%$ ), and highest for the QB classifications (at 20.9\%). The multi-date TM classifications had exhibited the largest proportional increases in mean $E_{\mathrm{n}}$ when aggregating from the pixel level to the field level, relative to the other image set classifications, while the QB classifications exhibited the smallest proportional increases (Table 4.1). This suggests that, unlike the field-based approach versus the independent pixel-based approach, where differences in variance corresponded to differences in per-pixel $E_{\mathrm{n}}$, these differences for the field-based approach versus the homogenous field-based approach corresponded with $E_{\mathrm{n}}$ increases associated with aggregation of probabilities to the field level.

Looking at the independent pixel-based SHDI distributions (Table 4.4; visible in Fig. 4.7), variance was much lower for the QB classifications (average variance of 
$2.64 \times 10^{-7}$ ) than for the single or multi-date TM classifications (average variances of $3.59 \times 10^{-5}$ and $1.92 \times 10^{-5}$ respectively), despite the fact that the QB classifications had higher $E_{\mathrm{n}}$, overall (Table 4.1). Variance in the simulated SHDI distributions based on the two field-based approaches corresponded better with the degree of entropy in the image set classifications. For the field-based and homogenous field-based simulation approaches, average variance was highest for the QB classifications $\left(1.78 \times 10^{-3}\right.$ and $8.71 \times 10^{-3}$ respectively), followed by the single date TM classifications $\left(1.29 \times 10^{-3}\right.$ and $7.27 \times 10^{-3}$ respectively), and then the multi-date TM classifications $\left(3.22 \times 10^{-4}\right.$ and $4.39 \times 10^{-3}$ respectively). Variance in the simulated SHDI distributions based on the fieldbased and homogenous field-based approaches corresponded better with the degree of entropy in the image set classifications, relative to the independent pixel-based approach.

Standard Z-scores are provided in Table 4.5, below, for comparing SHDI distribution offset from the reference values (Fig. 4.7). Looking at the absolute value of the Z-scores, there was a clearly visible difference between simulation approaches, with the largest values for the independent pixel based distributions, and lowest values for the for the homogenous field-based distributions. 
Table 4.5 Standard $(Z)$ scores for the reference SHDI values relative to the simulated SHDI distributions by image set classification, simulation approach, and landscape. Scores less than 1.96 are bolded.

\begin{tabular}{|c|c|c|c|c|c|}
\hline \multirow{2}{*}{$\begin{array}{l}\text { Image set } \\
\text { classification }\end{array}$} & \multirow{2}{*}{$\begin{array}{l}\text { Simulation } \\
\text { approach }\end{array}$} & \multicolumn{4}{|c|}{ Standard scores by landscape } \\
\hline & & $\begin{array}{c}\text { Landscape } \\
\text { A } \\
\end{array}$ & $\begin{array}{c}\text { Landscape } \\
\text { B } \\
\end{array}$ & $\begin{array}{c}\text { Landscape } \\
\text { C } \\
\end{array}$ & $\begin{array}{c}\text { Landscape } \\
\text { D } \\
\end{array}$ \\
\hline \multirow{4}{*}{$\begin{array}{c}\text { Single date } \\
\text { TM } \\
\text { classification }\end{array}$} & $\begin{array}{l}\text { Independent } \\
\text { pixel-based }\end{array}$ & -10.44 & -32.34 & -39.49 & -12.06 \\
\hline & & & & & \\
\hline & Field-based & -1.77 & -6.13 & -8.12 & -1.44 \\
\hline & $\begin{array}{l}\text { Homogenous } \\
\text { field-based }\end{array}$ & -0.0514 & -1.64 & -2.08 & -0.144 \\
\hline \multirow{4}{*}{$\begin{array}{c}\text { Multi-date } \\
\text { TM } \\
\text { classification }\end{array}$} & $\begin{array}{l}\text { Independent } \\
\text { pixel-based }\end{array}$ & -11.26 & -12.69 & -4.46 & -10.51 \\
\hline & & & & & \\
\hline & Field-based & -5.10 & -3.08 & -1.43 & -1.82 \\
\hline & $\begin{array}{l}\text { Homogenous } \\
\text { field-based }\end{array}$ & -0.489 & -0.372 & 0.501 & 0.177 \\
\hline \multirow{3}{*}{$\begin{array}{c}\text { QB } \\
\text { classification }\end{array}$} & $\begin{array}{l}\text { Independent } \\
\text { pixel-based }\end{array}$ & -546.45 & -1018.71 & -491.62 & -884.94 \\
\hline & Field-based & -6.98 & -8.55 & -6.17 & -10.57 \\
\hline & $\begin{array}{l}\text { Homogenous } \\
\text { field-based }\end{array}$ & -2.43 & -3.14 & -2.18 & -3.81 \\
\hline
\end{tabular}

Comparing image sets in terms of absolute Z-score (Table 4.5; Fig. 4.7 provides a visual reference), the values, in most cases, were larger for the distributions based on QB classifications than those of the corresponding single date TM classifications (paired Wilcoxon test, one-sided $\mathrm{p}=0.0012$ ). Differences between the single and multi-date TM classifications were less significant. Absolute Z-scores appeared to be larger for the single date TM classifications than for the multi-date TM classifications, but the 
differences were not significant at $95 \%$ confidence (paired Wilcoxon test, one-sided $\mathrm{p}=$ 0.0647). However, the SHDI distributions based on the homogenous field-based simulation of the multi-date TM classifications were the only distributions for which the reference SHDI values fell within the $95 \%$ confidence limits, across all four landscapes.

Finally, as seen in Figure 4.7, above, all of the distribution medians were offset from the SHDI value of their corresponding hardened classifications. While the offset was very slight in some cases (e.g. Landscape A, single date TM distributions), all of the hardened classification SHDI values were less than their corresponding distribution median, even with the homogenous field-based simulation approach, where within field structure was preserved across realizations. This offset is discussed further following the Results, particularly with respect to spatial autocorrelation between fields.

\subsubsection{Simulation convergence}

Figure 4.8, below, shows the cumulative variance in simulated SHDI versus the number of realizations generated for each of the landscapes, image set classifications, and simulation approaches. These are presented for the purpose of showing simulation convergence, as described in Section 2.5.1. 


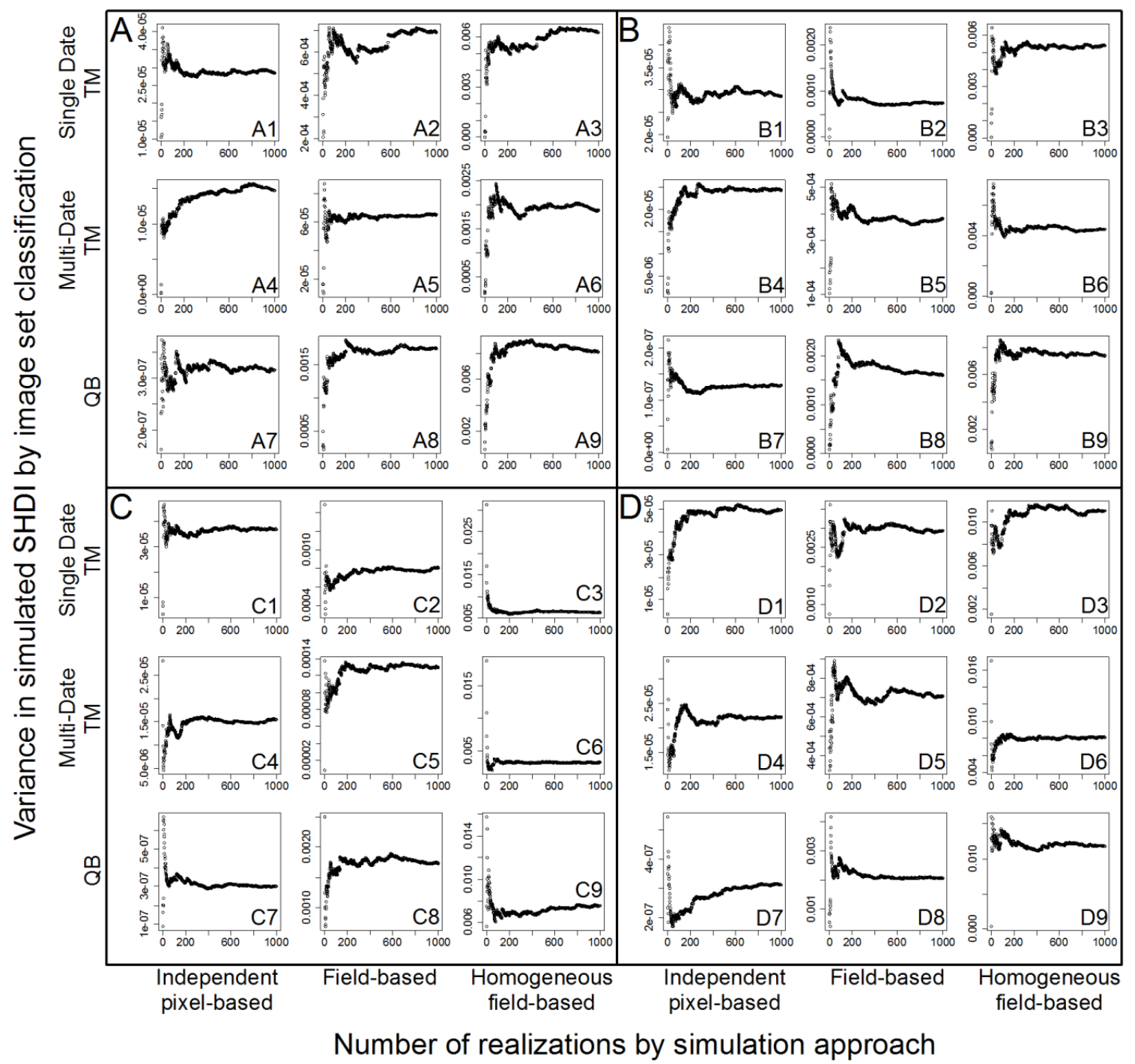

Figure 4.8 Cumulative variance in the simulated SHDI distributions versus the number of realizations. Plots are shown for (A) Landscape A, (B) Landscape B, (C) Landscape C, and (D) Landscape D.

Most of the simulations had roughly converged at around 400 realizations, showing fewer major shifts in variance with subsequent realizations. There were, however, some cases where moderate shifts or trends occurred after realization 400. For example, the plot for the field-based simulation of the single date TM classification of Landscape A (Fig. 4.8 A2) shows a large shift towards greater variance at approximately 
600 realizations. This case was caused by a rare event wherein two realizations with a very high proportion of Corn (causing lower than average SHDI) occurred in a row, suddenly increasing the overall variance.

Between realizations 800 to 1000 , most plots had reasonably levelled off and any visible trends were fairly minor, relative to those seen in the first 400 realizations. No clear differences were apparent amongst the simulation approaches or image set classifications in terms of how many realizations were required for convergence.

\subsection{Effects of positional and thematic uncertainties on the biodiversity models}

The results of the analysis of the effect of positional and thematic uncertainties on the biodiversity model coefficients (Section 3.4), aimed at addressing research questions 2 and 3 (Section 1.3), are presented in this section. The results of the image classification for the full, year-2011 study area are presented and characterized in terms of hard accuracy and $E_{\mathrm{n}}$ (Section 4.2.1). Examples of LC map realizations are presented (Section 4.2.2), along with corresponding examples of P.AG, MFS, and SHDI distributions (Section 4.2.3). Finally, the influence of the positional and thematic uncertainties on the biodiversity model coefficients are presented (Section 4.2.4). It should be noted that all results initially presented here are within the context of the independent field simulation approach (up to Section 4.2.3.2). These results are then compared with results from the locally-grouped and landscape-grouped approaches (Section 3.4.4.2). 


\subsubsection{Image classification}

This section presents characteristics related to the soft probability estimates from the Random Forest classification of the year-2011 TM imagery (Sections 2.3.1.2 and 3.4.3), as well as the accuracy associated with the hardened classification. First, the accuracy of the hardened classification, along with the error matrix, is provided (Section 4.2.1.1). Then, to better characterize the relationship between the thematic uncertainty model and error in the hard maps, $E_{\mathrm{n}}$ is compared between fields that have been correctly and incorrectly classified (Section 4.2.1.2). Finally, a closer look is taken at the incorrectly classified fields, focusing on how well second and third most-likely classes represented the reference class (Section 4.2.1.3).

\subsubsection{Accuracy of the hardened classification}

The accuracy metrics of the hardened classification (Section 3.4.3) are shown in Table 4.6, along with the corresponding error matrix in Table 4.7. This classification had an overall accuracy of $83.4 \%$ and a Cohen's kappa coefficient of 0.779 . Corn and Soybean were accurately classified with user's and producer's accuracies (Section 2.4.1) greater than $90 \%$, with most of the errors in these classes arising from confusion amongst themselves. Cereals had a high producer's accuracy (93.9\%) but a lower user's accuracy (63.1\%), due mainly to some Hay or Fallow fields being classified as Cereals. Hay, Pasture, and Legumes had low to moderate user's and producer's accuracies, ranging from the $45.1 \%$ to $75.9 \%$. Fallow had very low user's $(29.4 \%)$ and producer's $(30.3 \%)$ accuracies. Most of the misclassified area for the grass-like classes (Hay, Pasture, Legumes, and Fallow) was the result of confusion amongst themselves. 
Table 4.6 User's accuracy, producer's accuracy, overall accuracy, and Cohen's kappa coefficient for the hardened classification.

\begin{tabular}{cccccccc}
\hline & \multicolumn{7}{c}{ Accuracy by class (\%) } \\
\cline { 2 - 7 } & Corn & Soybean & Hay & Pasture & Legumes & Cereals & Fallow \\
\hline User's Accuracy & 93.0 & 92.0 & 72.6 & 73.1 & 52.6 & 63.1 & 29.4 \\
$\begin{array}{c}\text { Producer's } \\
\text { Accuracy }\end{array}$ & 92.3 & 94.8 & 66.7 & 75.9 & 45.1 & 93.9 & 30.3 \\
\hline Overall accuracy: & $\mathbf{8 3 . 4} \%$ & Cohen's kappa coefficient: & $\mathbf{0 . 7 7 9}$ & \\
\hline
\end{tabular}

Table 4.7 Error matrix for the hardened classification. Correctly classified area is bolded.

\begin{tabular}{|c|c|c|c|c|c|c|c|}
\hline \multirow{2}{*}{ Classification } & \multicolumn{7}{|c|}{ Reference $\left(\mathrm{km}^{2}\right)$} \\
\hline & Corn & Soybean & Hay & Pasture & Legumes & Cereals & Fallow \\
\hline Corn & 8.650 & 0.415 & 0.109 & 0.039 & 0 & 0.026 & 0.064 \\
\hline Soybean & 0.710 & 9.517 & 0.076 & 0.016 & 0 & 0.022 & 0 \\
\hline Hay & 0 & 0 & 3.462 & 0.333 & 0.862 & 0 & 0.110 \\
\hline Pasture & 0.009 & 0.006 & 0.443 & 1.601 & 0.101 & 0 & 0.031 \\
\hline Legumes & 0 & 0.041 & 0.762 & 0.016 & 0.909 & 0 & 0 \\
\hline Cereals & 0 & 0.057 & 0.247 & 0 & 0 & 0.735 & 0.126 \\
\hline Fallow & 0 & 0.007 & 0.090 & 0.105 & 0.143 & 0 & 0.143 \\
\hline
\end{tabular}




\subsubsection{Normalized entropy}

Figure 4.9, below, represents a subset of 12 of the 46 LCs from year 2011 (subset selected to show LCs with a range of P.AG, MFS, and SHDI values), showing $E_{\mathrm{n}}$, ground referenced crop types, and fields that have been misclassified in the hardened classification. In these examples, fields that had a low $E_{\mathrm{n}}$ tended to be correctly classified, whereas incorrectly classified fields tended to have moderate to high $E_{\mathrm{n}}$. However, there were several cases where high $E_{\mathrm{n}}$ fields were correctly classified. 

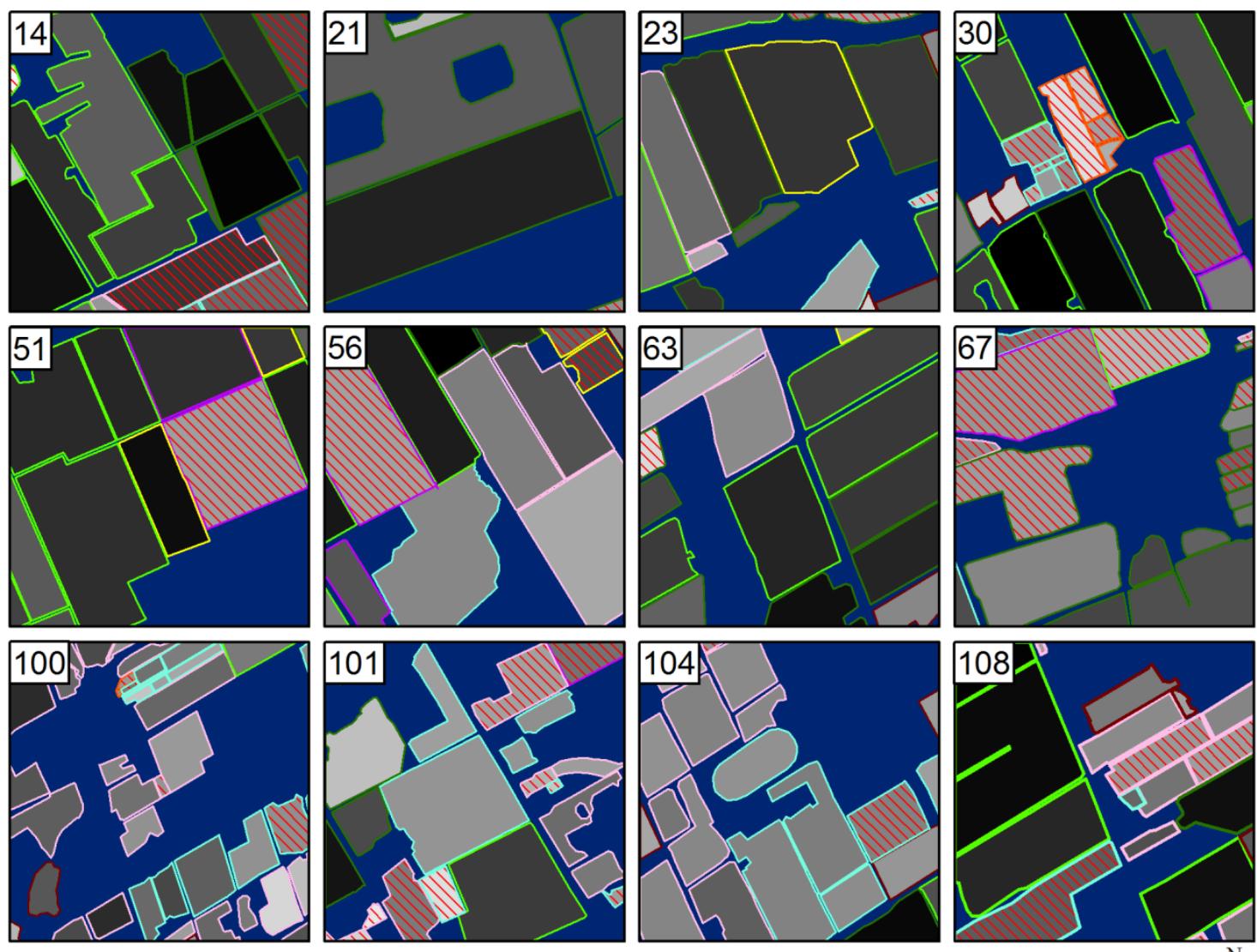

02004006008001,000

\section{Legend}

Ground referenced crop type:

\begin{tabular}{|l|l}
\hline & Corn \\
& Soybean \\
& Hay
\end{tabular}
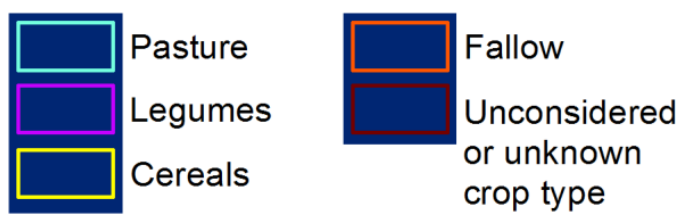

Normalized

entropy:

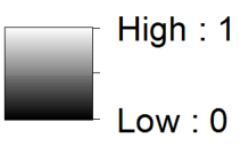

Incorrectly classified field (unconsidered and unknown crop types not included)

Figure 4.9 Normalized entropy $\left(E_{\mathrm{n}}\right)$ for a set of the LCs from year 2011. The

LCs are numbered, corresponding with the numbering system used in the Farmland Biodiversity Project.

Table 4.8, below, shows mean normalized entropies by class, and correctly versus incorrectly classified fields. Corn and Soybean fields had the lowest mean $E_{\mathrm{n}}$, followed 
by Cereals. Hay, Pasture, and Legume fields had moderate mean $E_{\mathrm{n}}$, followed by Fallow fields, with a higher mean $E_{\mathrm{n}}$. For all classes, mean $E_{\mathrm{n}}$ was higher for misclassified fields. However, the difference in mean $E_{\mathrm{n}}$ between correctly and incorrectly classified fields varied between classes. Corn, Soybean, Legumes, and Fallow had larger differences, while Hay, Pasture, and Cereals had lower differences. This suggests that higher mean $E_{\mathrm{n}}$, as a measure of ambiguity in class assignment (Gonçalves et al. 2012), may be more associated with error for some classes (e.g. Corn and Soybean) than for others (e.g. Hay and Pasture). Some of those classes where higher mean $E_{\mathrm{n}}$ was less associated error had shown separability issues in the limited extent classifications (Appendix C).

Table 4.8 Area weighted mean normalized entropy $\left(E_{\mathrm{n}}\right)$ values by ground referenced class. Polygons representing alternate boundaries were not considered.

\begin{tabular}{cccccccc}
\hline & \multicolumn{7}{c}{ Area weighted mean normalized entropy } \\
\cline { 2 - 7 } & Corn & Soybean & Hay & Pasture & Legumes & Cereals & Fallow \\
\hline Overall & 0.216 & 0.244 & 0.518 & 0.497 & 0.436 & 0.339 & 0.685 \\
$\begin{array}{c}\text { Correctly } \\
\text { classified } \\
\text { fields }\end{array}$ & 0.203 & 0.232 & 0.483 & 0.492 & 0.323 & 0.338 & 0.558 \\
$\begin{array}{c}\text { Incorrectly } \\
\text { classified } \\
\text { fields }\end{array}$ & 0.411 & 0.476 & 0.583 & 0.523 & 0.525 & 0.369 & 0.732 \\
\hline
\end{tabular}

\subsubsection{Second and third most-likely classes of incorrectly classified fields}

Table 4.9, below, shows the total area of incorrectly classified (hardened classification) fields, along with the area of incorrectly classified fields that have a correct 
second most-likely or third most-likely class. Most of the $4.97 \mathrm{~km}^{2}$ of incorrectly hardclassified crop area had a correct second-most likely class, at $66.5 \%$, though a sizeable proportion remained incorrectly represented by these classes. Considering the second and third-most likely classes, $80.2 \%$ of incorrectly classified crop area was represented, leaving a total 42 fields (of 200 incorrectly classified fields) for which their ground reference class did not match their three most-likely classes.

Table 4.9 Area of incorrectly classified fields that have a correct second most-likely or third most-likely class. The area of incorrectly classified fields is included. Polygons representing alternate boundaries were not considered.

\begin{tabular}{|c|c|c|c|c|}
\hline \multirow[b]{2}{*}{$\begin{array}{l}\text { Reference } \\
\text { class }\end{array}$} & \multirow{2}{*}{$\begin{array}{l}\text { Incorrectly } \\
\text { classified } \\
\text { area }\left(\mathrm{km}^{2}\right)\end{array}$} & \multicolumn{2}{|c|}{ Area of incorrectly classified area with: } & \multirow{2}{*}{$\begin{array}{l}\text { Total of correct } \\
\text { second or third } \\
\text { most-likely }\left(\mathrm{km}^{2}\right)\end{array}$} \\
\hline & & $\begin{array}{l}\text { correct second most- } \\
\text { likely class }\left(\mathrm{km}^{2}\right)\end{array}$ & $\begin{array}{l}\text { correct third most- } \\
\text { likely class }\left(\mathrm{km}^{2}\right)\end{array}$ & \\
\hline Corn & 0.719 & 0.656 & 0.059 & 0.715 \\
\hline Soybean & 0.526 & 0.368 & 0.126 & 0.494 \\
\hline Hay & 1.726 & 1.099 & 0.187 & 1.286 \\
\hline Pasture & 0.509 & 0.282 & 0.195 & 0.477 \\
\hline Legumes & 1.105 & 0.822 & 0.053 & 0.874 \\
\hline Cereals & 0.047 & 0.014 & 0.033 & 0.047 \\
\hline Fallow & 0.330 & 0.063 & 0.025 & 0.087 \\
\hline Overall & 4.965 & 3.303 & 0.678 & 3.981 \\
\hline
\end{tabular}

Over $90 \%$ of incorrectly classified Corn, Soybean, Pasture, and Cereals crop areas were correctly represented by their second or third most-likely classes. Hay and Legumes, which together accounted for $57 \%$ of the overall incorrectly classified crop 
area, had $74.5 \%$ and $79.1 \%$ of their incorrectly classified areas represented correctly by their second or second-or-third most-likely classes, respectively. Fallow, while accounting for a lower amount of the incorrectly classified area, only had $26.4 \%$ of its incorrectly classified area represented by its second or third most-likely classes.

Looking case-by-case at fields that were not correctly represented by any of their three most-likely classes revealed that many were either uncertainly assigned a ground referenced class (determined by ground reference team comments), fairly atypical cases of their ground referenced class (e.g. overgrown by grass or weeds, showing large amounts of exposed soil, etc.; based on interpretation of the 2011 air photos), or perhaps assigned an incorrect or non-representative ground reference class (e.g. ground referencing error or changes in crop type within the growing season). Examples of some of these cases are provided in Appendix G. Hay and Legumes had more of these cases. For example, of the 10 largest ground referenced Hay fields that weren't represented by their three most-likely classes, 8 had reasonably questionable ground referenced classes or very poor crop condition (two examples in Appendix G). If these 8 fields were to be removed from consideration, the proportion of incorrectly classified Hay fields, with a correct second most-likely class, would change from $63.7 \%$ to $80.4 \%$, and the proportion for the correct second or third most-likely class would change from $74.5 \%$ to $94.0 \%$.

\subsubsection{LC map realizations}

LC map realizations (error-sensitized vertex positions, alternate boundaries, and thematic classes) were generated primarily as tables (based on records of error-sensitized 
area, boundary interpretation probability, and thematic class probabilities; Section 3.4.4.1), without actually producing a visual representation of each. This section provides reconstructed visual examples and analysis of these realizations. Due to the fact that variation arising from the three uncertainty sources can be more easily viewed using

different visualization approaches, realization examples of alternate boundaries and thematic classes (Section 4.2.2.1) are shown separately from those of vertex position (Section 4.2.2.2).

\subsubsection{Alternate boundaries and thematic classes}

Figure 4.10, below, shows an example of a hard LC map (reference boundaries with year-2011 crop types based on hard image classification) with three realizations of field boundaries (sensitized vertices not shown) and thematic classes. Some cases where changes in boundaries occur are circled and numbered. Cases 1 and 3 (Fig. 4.10 C, D) are examples of where a field in the hard LC map has been split along a potential edge. Case 2 (Fig. $4.10 \mathrm{C}$ ) is an example of where a field has been split along a potential edge, with a split portion being taken out of consideration as an active field. Case 4 (Fig. 4.10 D) is an example of where a field has been merged. Finally, cases 5 and 6 (Fig. 4.10 D) show examples where potential fields have been entered into consideration. 

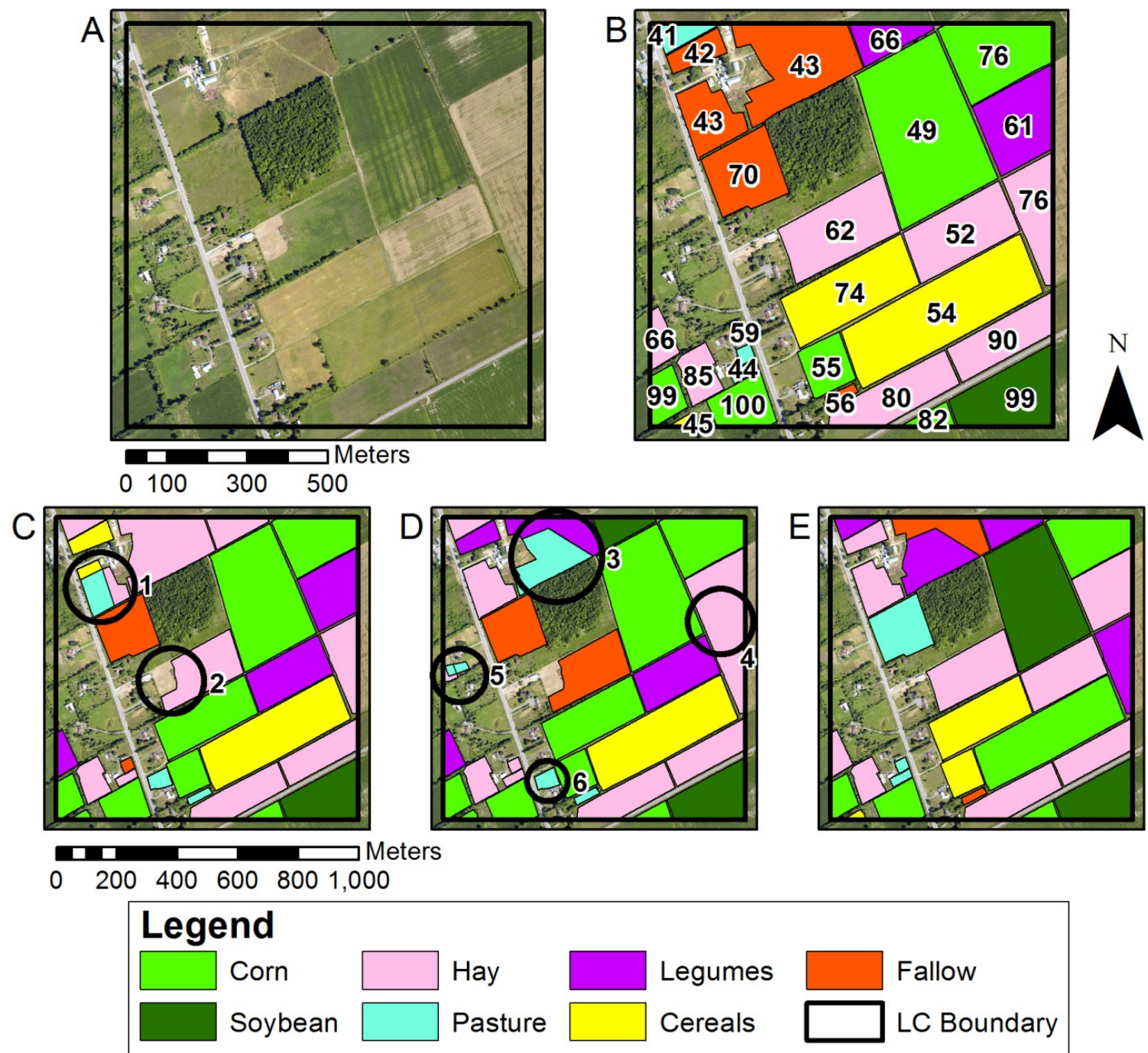

Figure 4.10 Examples of realizations of alternate LC map composition and configuration. Shown are (A) the air photo of the LC, (B) the hard LC map with most-likely classes assigned to fields (numbers represent the probability estimate for the most-likely class, as a percentage), and (C, D, E) realizations of alternate LC map composition and configuration. Vertex position realizations are not shown. For these examples, the PRNs used to simulate alternate field boundaries were altered in order to show more examples across just three realizations. 
The probabilities of the most-likely classes are shown in Figure $4.10 \mathrm{~B}$. Examples of fields that were assigned a class with higher confidence (larger value for the most-likely class) can be seen near the southeast corner of the LC. Examples of lower confidence in class assignment can be seen near the northwestern corner of the LC. Those fields that had been assigned a class with lower confidence showed more variation in class across the three realizations than those with more confidently assigned classes.

\subsubsection{Alternate vertex positions}

Figure 4.11, below, shows 10 realizations of sensitized vertex positions for polygons representing fields in the southeastern portion of the LC shown in Figure 4.10. Polygons representing alternate field boundaries are included. An example of the difference in boundary positioning due to boundary interpretation uncertainty versus vertex position uncertainty is circled.

A few issues with the vertex position simulation approach can be seen in Figure 4.11, below. In some cases, there was overlap between adjacent, sensitized field polygons. Also, reference field polygons that were flush with the LC boundary in the hard map would extend slightly past the boundary or stop slightly short of it when sensitized (see fields at the southern and eastern sides of Fig. 4.11). Finally, portions of field polygons that had a high vertex density tended to have more irregularly shaped boundaries when sensitized, due to the fact that spatial autocorrelation in nearby vertices was not considered (Section 3.4.4.1). An example of this is shown in Figure 4.12, below. In Figure 4.12 A, straight edge boundaries with a relatively low vertex density are well 
preserved when the vertices are sensitized. In Figure 4.12 B, which has a higher vertex density, the somewhat straight edges become more irregular when sensitized.

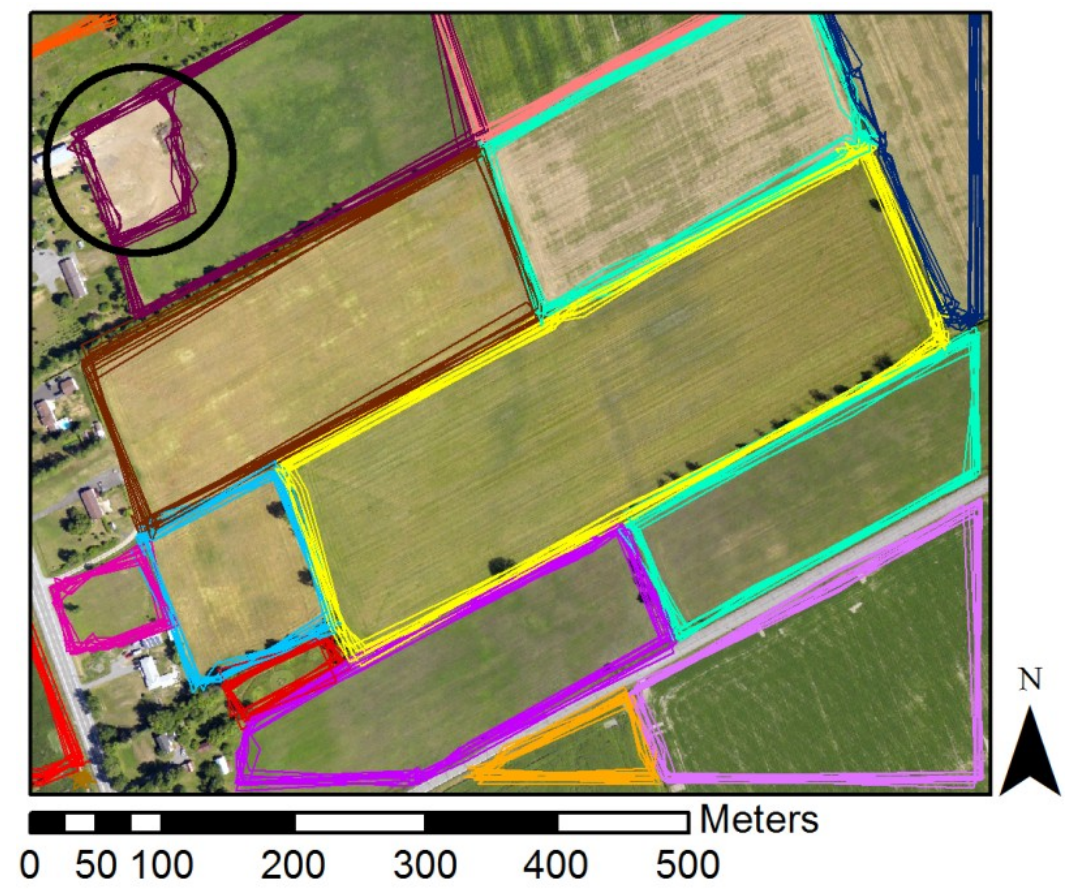

Figure 4.11 Ten realizations of field boundary vertex positions. The colours are just used to distinguish between different fields. An example of the difference between vertex position and boundary interpretation realizations is circled, for a field that had an alternate boundary representation. 

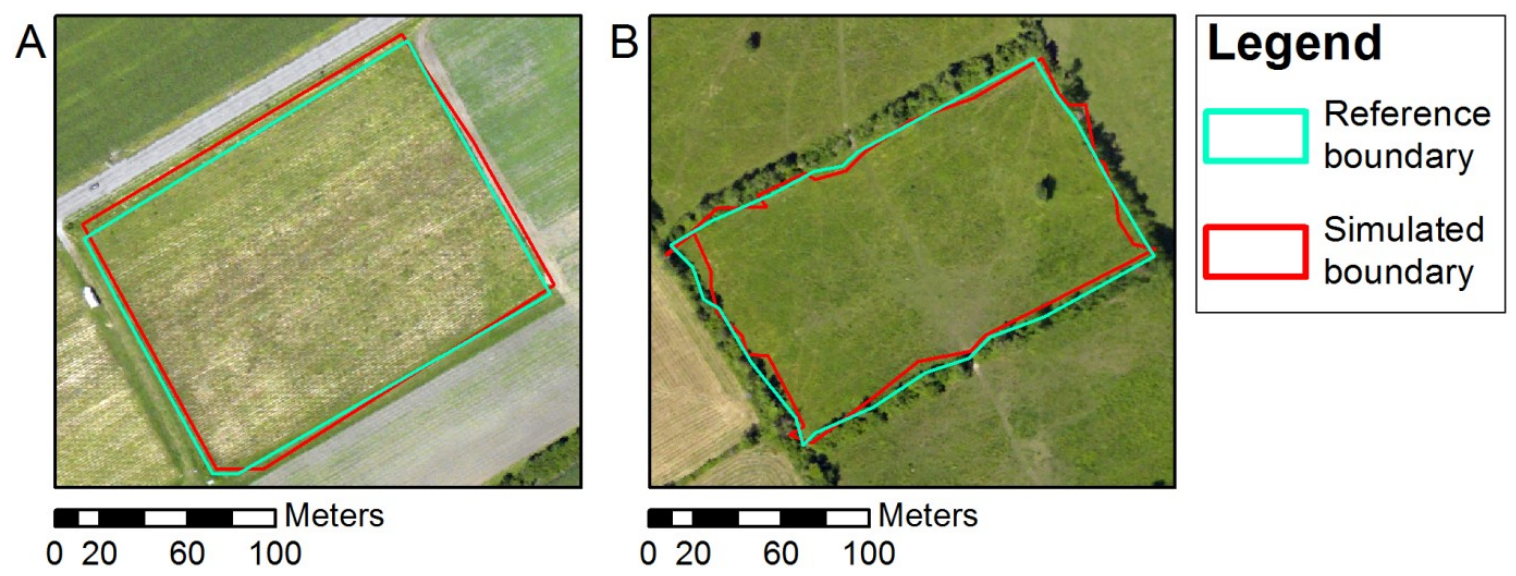

Figure 4.12 An example of the difference in shape preservation for simulated (errorsensitized) vertices of fields with (A) lower and (B) higher vertex densities.

Despite these issues, the variance in error-sensitized field boundaries (referring to vertex positions only, here) roughly corresponded with the variance across the redigitized set of field boundaries (Section 3.4.1). Figure 4.13, below, compares these variances. These data represent 44 of the 100 re-digitized fields that had consistent boundary interpretation and no major differences between the 2008 and 2011 air photos.

The RMSE between the simulated boundary variance and the corresponding redigitized boundary variance was $332.16 \mathrm{~m}^{2}$. Residuals were somewhat heteroscedastic, as fields that had larger variance tended to have more error. A tendency for the errorsensitized field boundaries to overestimate the variance in area is evident. Fitting a linear model on these data (regression line not shown in Fig. 4.13), with an intercept fixed at 0 $\mathrm{m}^{2}$, resulted in a coefficient of determination $\left(\mathrm{R}^{2}\right)$ of $0.951\left(\mathrm{R}^{2}=0.805\right.$, if intercept not fixed) and a regression coefficient of 1.186 as opposed to 1. 

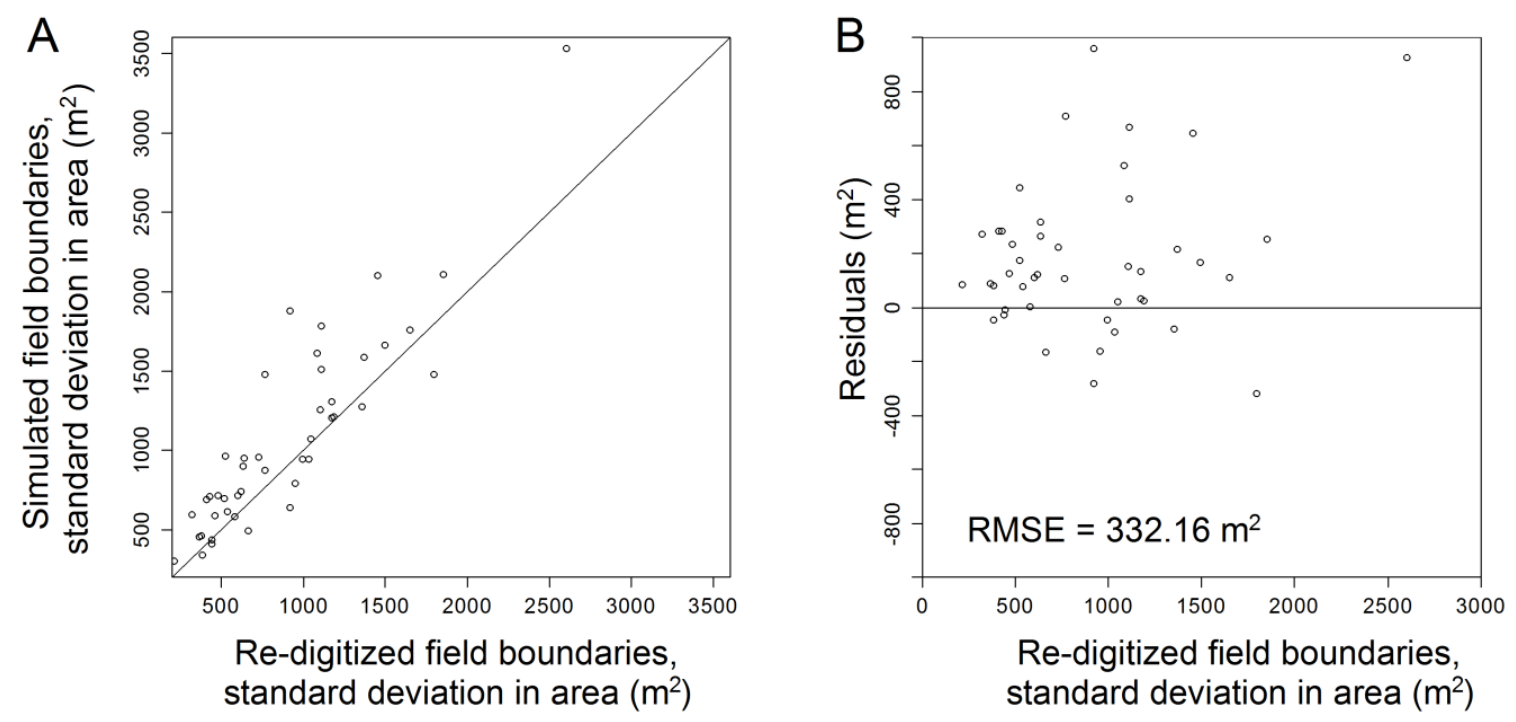

Figure 4.13 Comparison plots for simulated (error-sensitized) versus redigitized field boundaries. Included are, (A) a plot of the standard deviation in area of the simulated field boundaries (500 realizations) versus standard deviation in area of the corresponding re-digitized fields (24 re-digitized copies), and (B) a residual plot for the variance in area of the simulated boundaries versus the re-digitized field boundaries, relative to the ideal case, wherein they are equal. Alternate field boundary interpretations were not considered.

\subsubsection{Simulated metrics under independent field and grouped simulation}

Distributions representing the landscape metric realizations are provided in this section. Examples of output distributions from independent field simulation are presented (Section 4.2.3.1). Examples of LCs wherein simulated SHDI metrics are offset from the corresponding hard metrics are shown (Section 4.2.3.2). For these LCs, SHDI distributions generated under the grouped simulation approaches (Section 3.4.4.2) are provided. 


\subsubsection{Landscape metrics under independent field simulation}

Figures 4.14, 4.15, and 4.16, below, show the distributions of the simulated P.AG, MFS, and SHDI metrics, respectively, as generated under the independent field simulation approach (Section 3.4.4.2), for the same LC subset that was shown in Figure 4.9 .
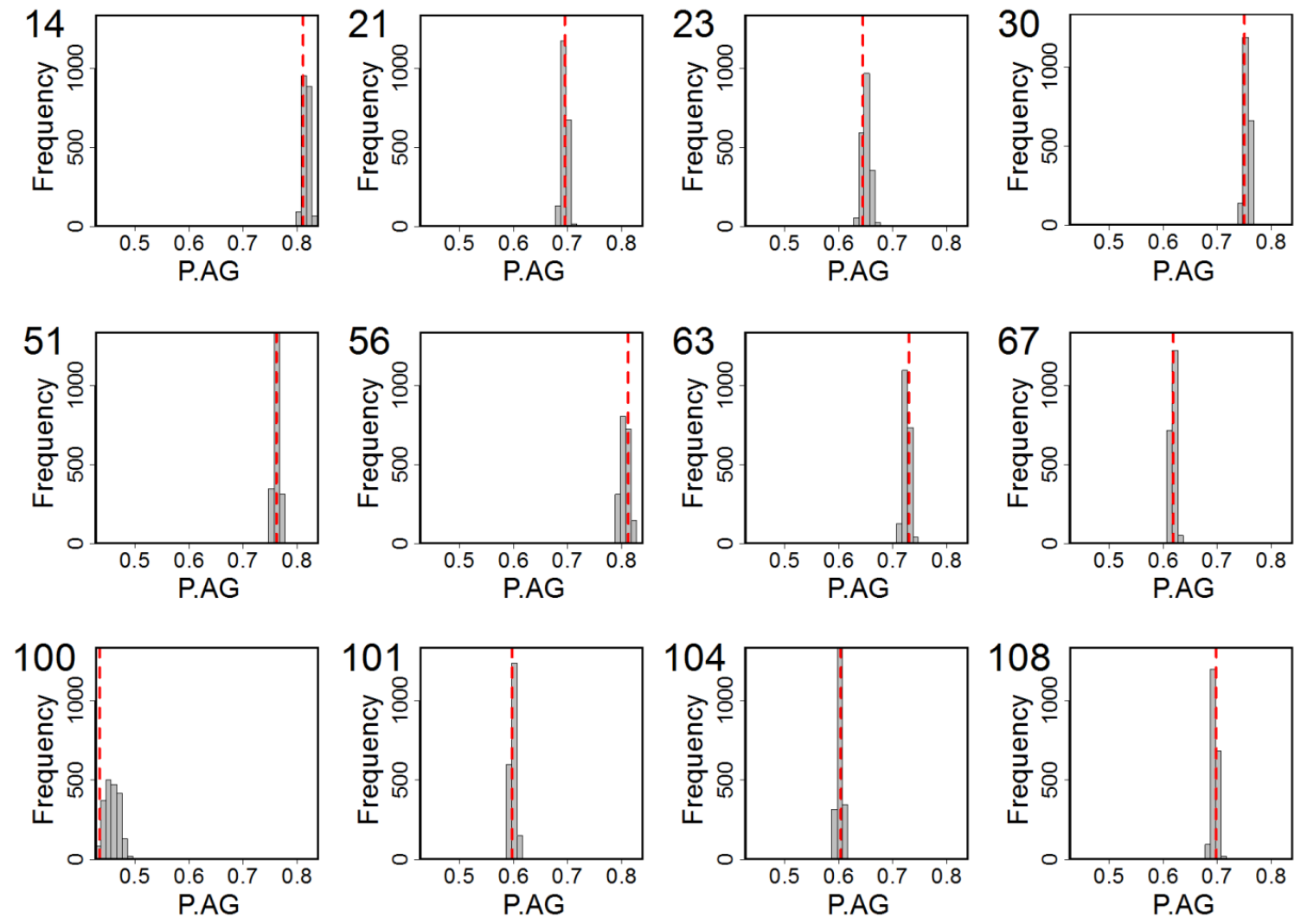

Figure 4.14 Distributions of simulated P.AG metrics. The distributions shown represent a subset of the year-2011 LCs. The LCs are numbered, corresponding with the numbering system used in the Farmland Biodiversity Project. The dashed red line represents the metric based on the hard LC map.

The distributions in Figure 4.14 suggest that the variances within the simulated P.AG metrics of individual LCs, arising from positional and thematic uncertainties in the 
LC maps (thematic uncertainty not from the classification, but related to cases where field boundaries were removed from consideration; Section 3.4.2.2) were much lower than the variance in P.AG metrics between LCs. Most of the simulated P.AG distributions were fairly normally distributed, with a few cases of moderate skewness. Shapiro Wilk tests, performed on 100 realizations of P.AG for each LC, found 6 of the 46 simulated P.AG distributions from year-2011 to be significantly non-normal (at $\alpha=0.05$ ).
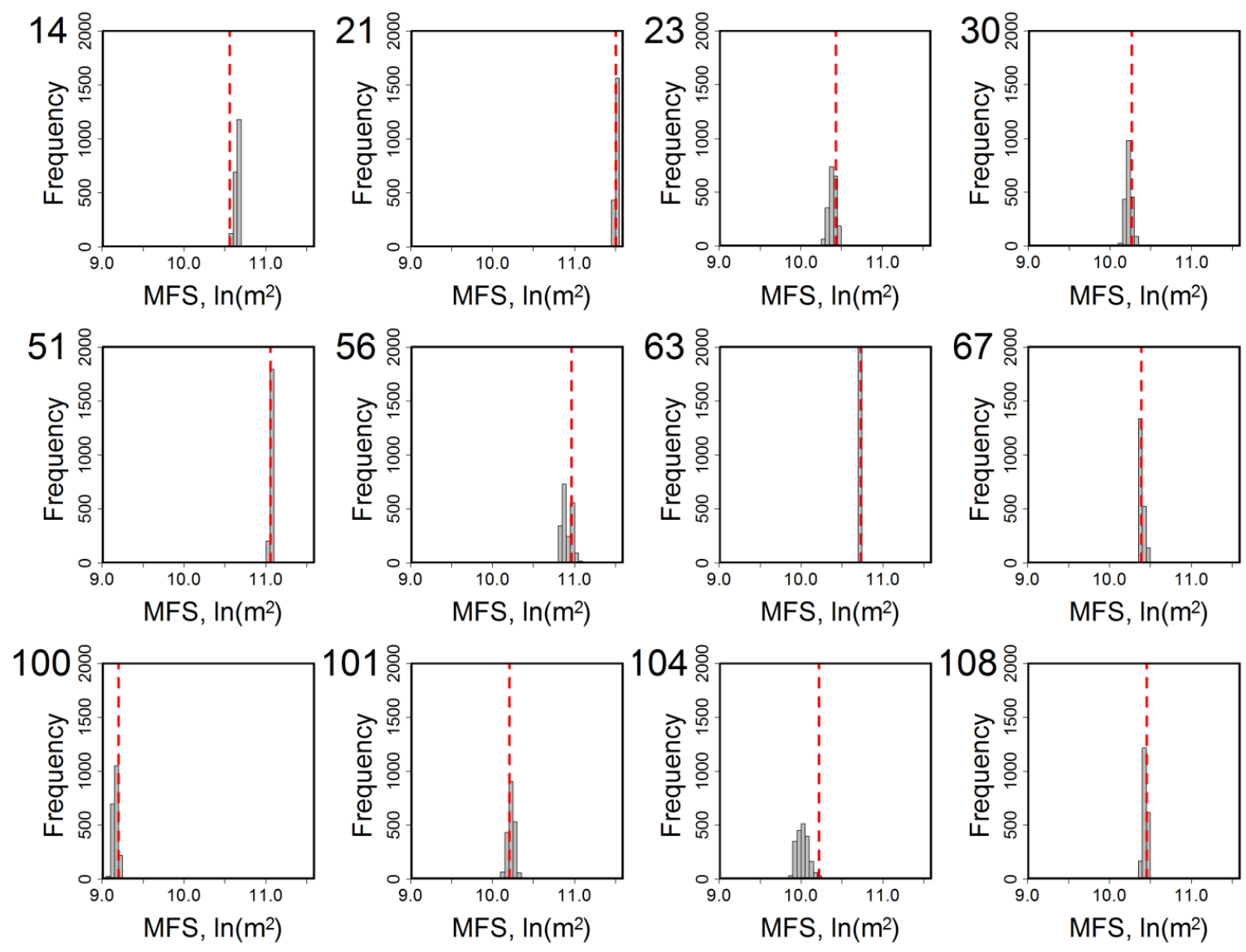

Figure 4.15 Distributions of simulated MFS metrics. The distributions shown represent a subset of the year-2011 LCs. The LCs are numbered, corresponding with the numbering system used in the Farmland Biodiversity Project. The dashed red line represents the metric based on the hard LC map. 
Figure 4.15 suggests that, like P.AG, the variance in the MFS metrics within individual LCs were much lower than the variance in these metrics between LCs. The simulated MFS distributions were more prone to multi-modality and skewness than the P.AG distributions. Shapiro Wilk tests (on 100 realizations, with $\alpha=0.05$ ) found 31 of the 46 simulated MFS distributions from year-2011 to be significantly non-normal.
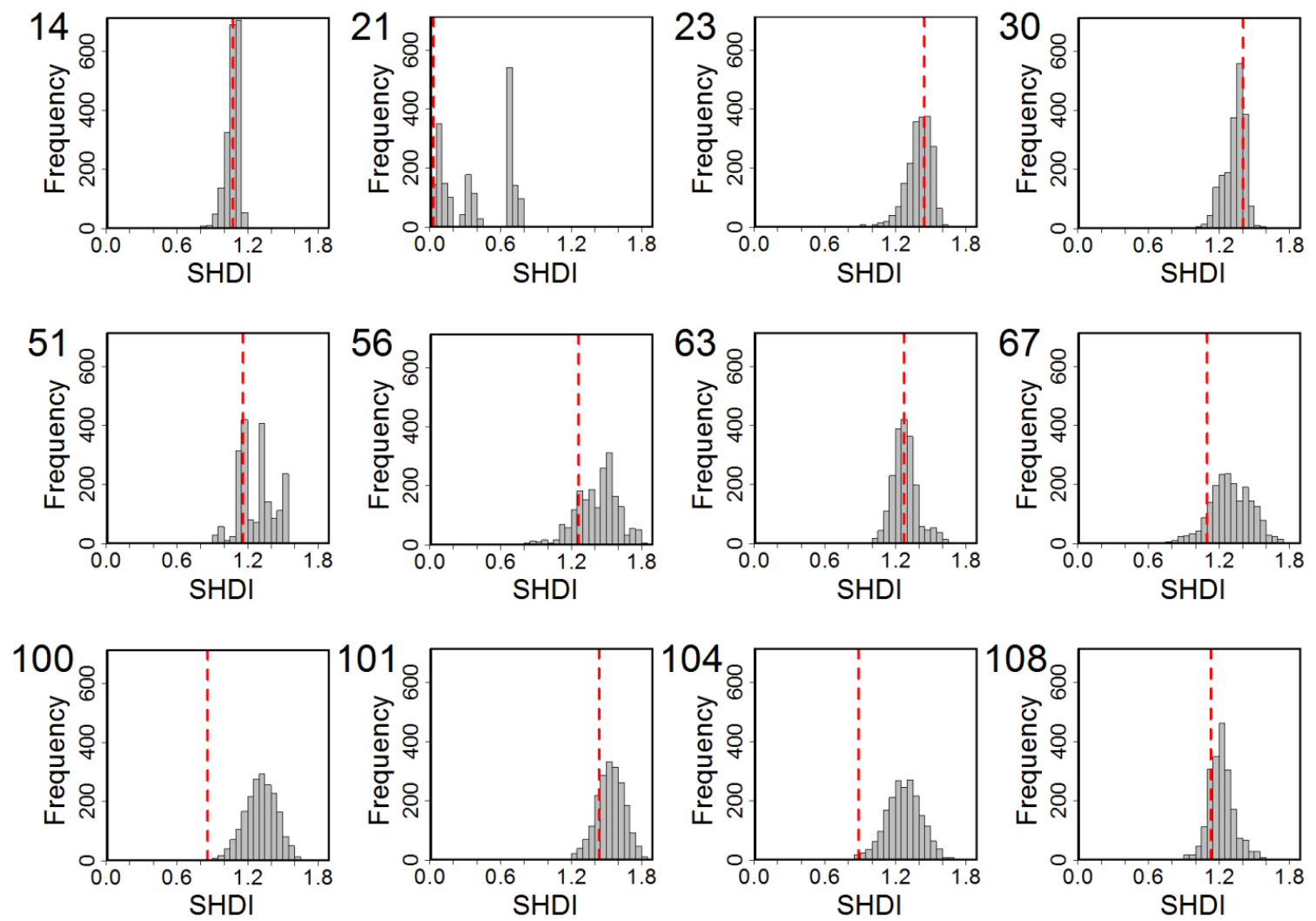

Figure 4.16 Distributions of simulated SHDI metrics. The distributions shown represent a subset of the year-2011 LCs. The LCs are numbered, corresponding with the numbering system used in the Farmland Biodiversity Project. The dashed red line represents the metric based on the hard LC map.

Compared to P.AG and MFS (Figs. 4.14 and 4.15), Figure 4.16 suggests that the variance in the SHDI metrics within individual LCs was closer to the variance in the 
SHDI metrics between LCs. These distributions were prone to multi-modality and skewness, with the Shapiro Wilk tests (on 100 realizations, with $\alpha=0.05$ ) determining 24 of the 46 distributions to be significantly non-normal.

There were several cases where the simulated SHDI metrics were offset from the SHDI metric of the hard LC maps, usually towards greater SHDI. Examples of this include LCs 21, 67, 100, and 104 (Fig. 4.16). Larger offsets tended to occur mainly in LCs characterized by several smaller fields, where one or two crop types dominate, and classes have been assigned with less certainty (see Fig. 4.17 in Section 4.2.3.2). These offsets were not limited to the simulated SHDI metrics, but occurred in them rather frequently, and to a greater extent, relative to the between LC variance. An example of this offset for P.AG is seen in LC 100 (Fig. 4.14). An example for MFS is seen in LC 104 (Fig. 4.15). Offsets for both the P.AG. and MFS cases tended to occur in LCs where there were several alternate field boundary representations (Section 3.4.2).

\subsubsection{Simulated SHDI offset and grouped simulation}

Figure 4.17, below, shows three hard LC maps for which the mean of the simulated SHDI distribution was offset from hard SHDI metric. LC 67 (Fig. $4.17 \mathrm{~A}$ ) is dominated by Corn, with some Soybean and Hay fields. Many of the fields were classified with low certainty, particularly the Hay fields in the northwestern corner and the small Corn and Soybean fields along the eastern boundary. LC 100 (Fig. 4.17 B) is characterized by several small Hay fields and small Pasture fields, which have been assigned classes with low to moderate certainty. LC 104 (Fig. 4.17 C) is similar to LC 100 , but with fewer and moderately larger fields 

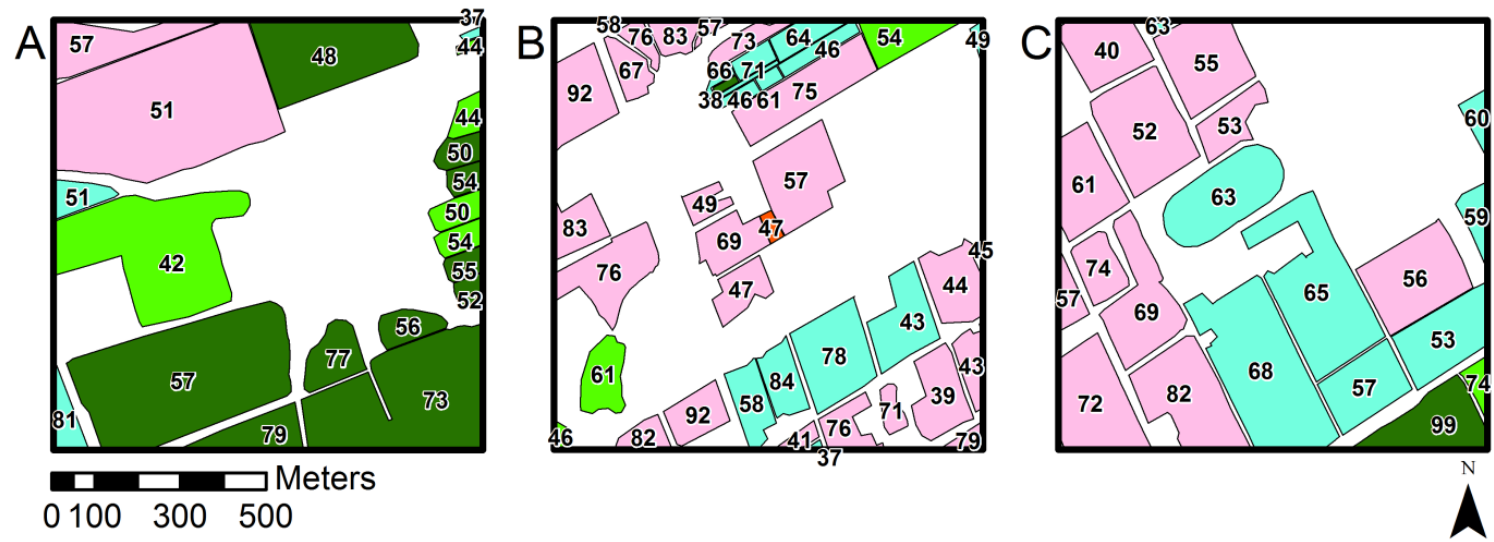

Legend

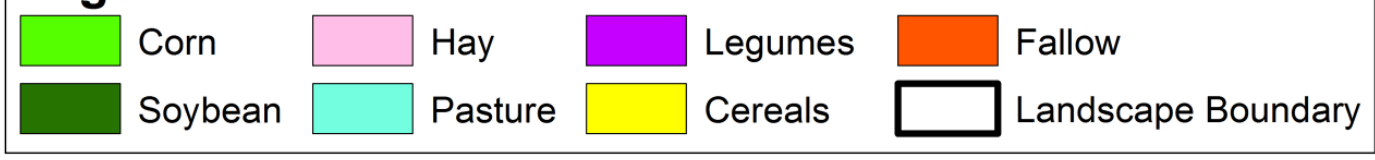

Figure 4.17 Hard LC maps for which there was an offset between hard SHDI metric and the mean of the simulated SHDI distribution. The maps represent (A) LC 67, (B) LC 100, and (C) LC 104. Their SHDI distributions are shown in Figure 4.16. The number assigned to each field represents the probability estimate (as a percentage) for its most-likely class.

The grouped simulation approaches (Section 3.4.4.2) tended to reduce the offset between the mean of the simulated SHDI metrics and the hard SHDI metric, though not equally across all LCs. Figure 4.18, below, compares these offsets for LCs 67, 100, and 104, using the independent field and grouped simulation approaches. The offsets were largest for independent field simulation. Locally-grouped simulation reduced the offset (relative to independent field simulation) by $52.3 \%, 5.8 \%$, and $49.5 \%$ for LCs 67,100 , and 104, respectively. Landscape-grouped simulation reduced the offset (relative to independent field simulation) by $85.3 \%, 45.2 \%$, and $78.5 \%$ for LCs 67,100 , and 104 , respectively. The simulated SHDI distributions became more irregular (skewed, with several local modes) as the degree of field grouping increased. 

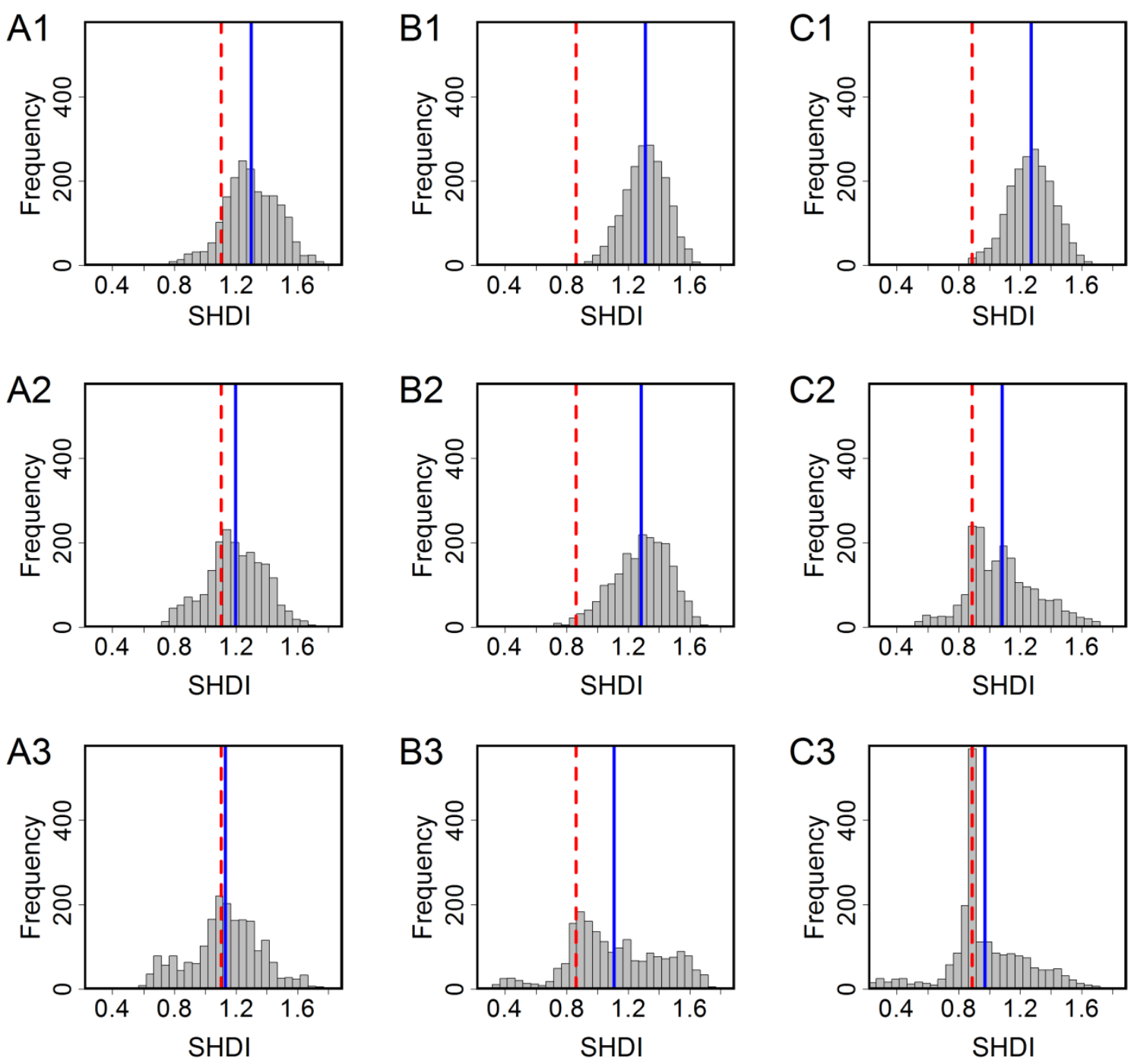

Figure 4.18 Offset between hard and simulated SHDI metrics under independent field and grouped simulation approaches. Histograms represent (A) LC 67, (B) LC 100, and (C) LC 104 when crop types has been simulated with (1) the independent field approach, (2) the locally-grouped approach, or (3) the landscape-grouped approach (Section 3.4.4.2). The dashed red line represents the hard SHDI metric, while the solid blue line represents the mean of the simulated SHDI distribution. 


\subsubsection{Simulated model coefficients}

This section provides results from the extension of the simulation through the modelling and model averaging analysis (Sections 2.6.1 and 3.4.4.3). $\widehat{\bar{\Theta}}_{\text {sim }}$ are first presented for the gamma diversity models, as produced under the independent field simulation approach. Results related to offset issues, both between the original coefficients and $\widehat{\bar{\Theta}}_{\text {hard }}$ (Section 4.2.4.1) and between $\widehat{\bar{\Theta}}_{\text {hard }}$ and $\widehat{\bar{\theta}}_{\text {sim }}$ (Section 4.2.4.2), are then presented. The uncertainties in model coefficients associated with the positional and thematic uncertainties, $\sigma_{\text {sim }}^{2}$, are then compared to the uncertainties associated with the sample, given the model, and model selection, $\widehat{\operatorname{var}}\left(\hat{\bar{\theta}}_{\text {sim }}\right)$ (Section 4.2.4.3). Finally, examples of convergence plots for $\widehat{\bar{\theta}}_{\text {sim }}$ and $\widehat{\operatorname{var}}\left(\hat{\bar{\theta}}_{\text {sim }}\right)$ are then presented (Section 4.2.4.4).

In addition to the terms defined in Section 3.4.4.3, throughout this section $\widehat{\boldsymbol{\beta}}_{\text {P.AG }}, \widehat{\boldsymbol{\beta}}_{\text {MFS }}$, and $\widehat{\boldsymbol{\beta}}_{\text {SHDI }}$ will be used to represent the model averaged coefficient estimates (obtained through AICc model averaging) for the P.AG, MFS, and SHDI variables, respectively (each of which may correspond to the original coefficient, $\widehat{\bar{\Theta}}_{\text {hard }}$, or $\left.\widehat{\widehat{\theta}}_{\text {sim }}\right)$.

Figure 4.19, below, shows the model averaged regression coefficients for the gamma diversity models, including coefficients based on the original metrics, coefficients based on the hard metrics (from the hard LC maps) ( $\left.\widehat{\bar{\Theta}}_{\text {hard }}\right)$, and coefficients based on the simulated metrics $\left(\widehat{\bar{\theta}}_{\text {sim }}\right)$, under the independent field simulation approach. The corresponding figures for all diversity types (including abundance) are included in

\section{Appendix H.}



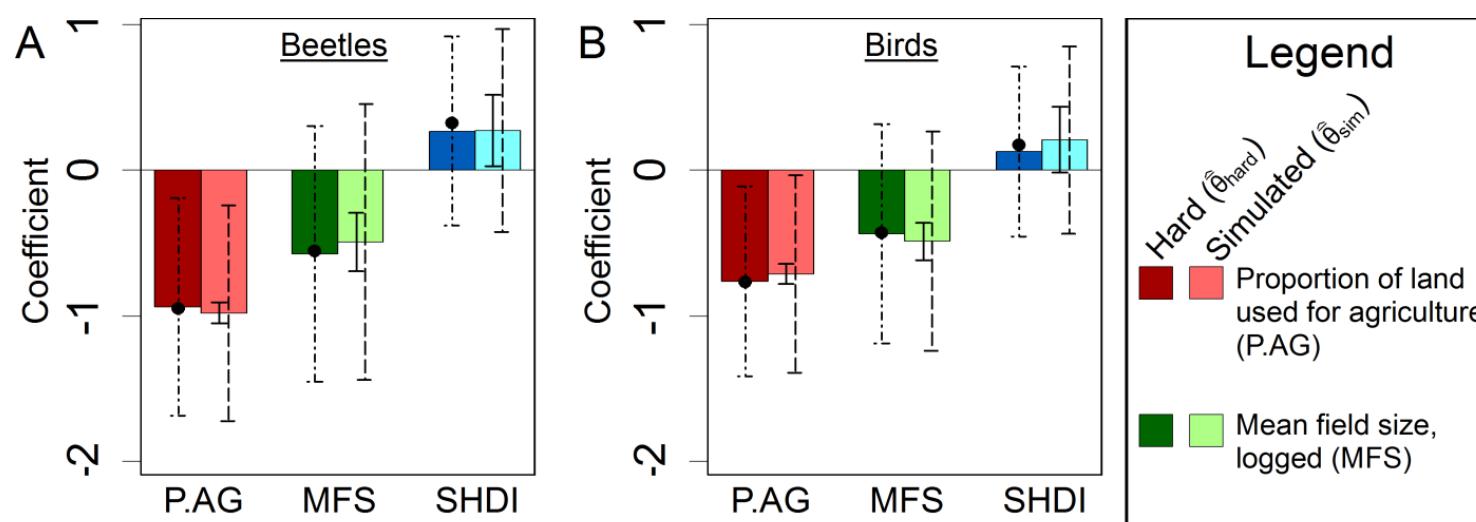
used for agriculture (P.AG)

Mean field size, logged (MFS)
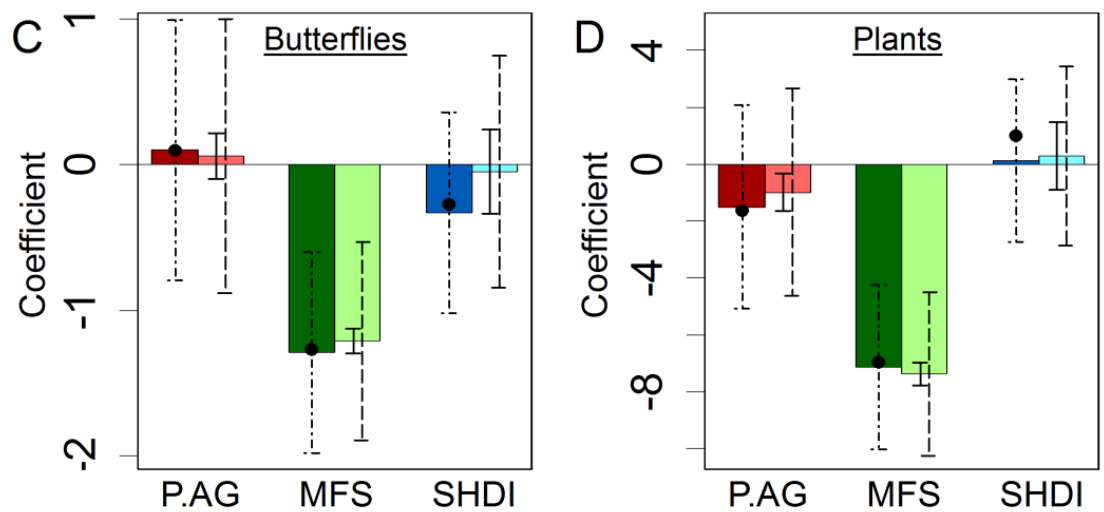

Shannon Diversity Index of crop types (SHDI)

- Coefficient based on the original dataset

7

95\% model averaging

i confidence interval

based on $\widehat{\operatorname{se}}\left(\hat{\theta}_{\text {hard }}\right)$

T $95 \%$ confidence interval based on standard deviation of simulated
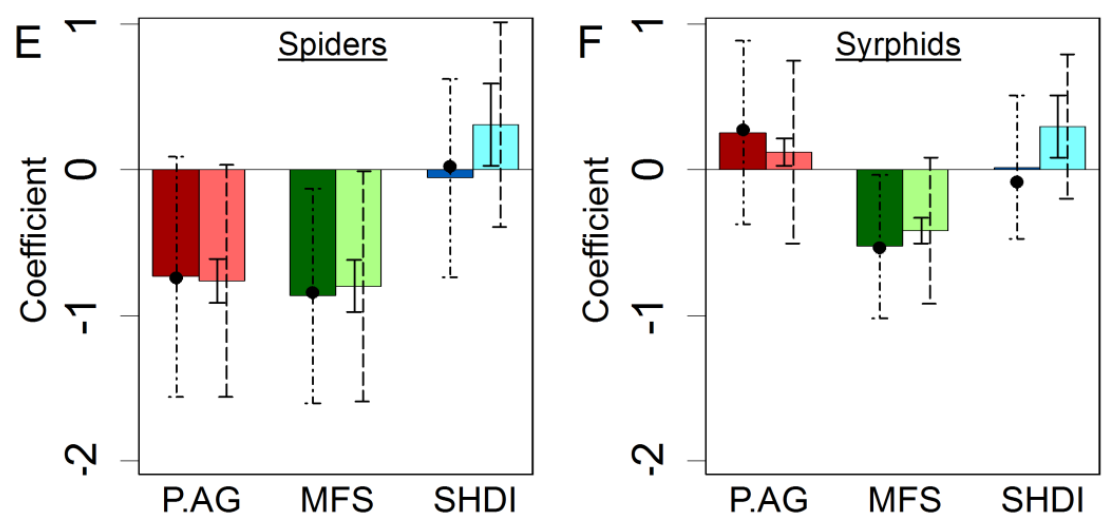

coefficients, $\sigma_{\text {sim }}$

$T$

95\% model averaging confidence interval

based on $\widehat{\operatorname{se}}\left(\hat{\theta}_{\mathrm{sim}}\right)$

Figure 4.19 Hard and simulated (independent field simulation approach) model averaged regression coefficients and confidence intervals for the (A) beetles, (B) birds, (C) butterflies, (D) plants, (E) spiders, and (F) syrphids gamma diversity models. 


\subsubsection{Offset between original and hard coefficients}

Differences between the coefficients based on the original dataset (metrics based on ground referenced maps in both 2011 and 2012) and $\widehat{\bar{\theta}}_{\text {hard }}$ were mainly confined to $\widehat{\bar{\beta}}_{\text {SHDI }}$. The difference for $\widehat{\bar{\beta}}_{\text {SHDI }}$ was larger for the beetles abundance model $(-0.59)$, the birds abundance model (0.49), the plants alpha, beta, and gamma models $(0.29,0.58$, and 0.87 respectively), the spiders abundance model (-0.13), and the syrphids gamma and abundance models $(-0.11$ and -0.11 respectively). For the remaining 15 biodiversity models (of 23 total) the difference was less than 0.1 .

\subsubsection{Offset between hard and simulated coefficients}

An issue with the independent field simulation approach can be seen for $\widehat{\bar{\beta}}_{\text {SHDI }}$ in Figure 4.19 C, E, F, above. Similar to the offset between the simulated SHDI metrics and the hard SHDI metric that was seen in Figure 4.18, $\widehat{\bar{\theta}}_{\text {sim }}$ are offset (more positive) from $\widehat{\bar{\theta}}_{\text {hard }}$. With the grouped simulation approaches, this offset was reduced, though not completely eliminated (Fig. 4.20, below). Looking at just the alpha, beta, and gamma diversity models (abundance models had larger coefficients and correspondingly larger

differences), the average difference between $\widehat{\bar{\theta}}_{\text {hard }}$ and $\widehat{\widehat{\theta}}_{\text {sim }}$ (for $\widehat{\bar{\beta}}_{\text {SHDI }}$ ) was -0.132 with independent field simulation, -0.070 with locally-grouped simulation, and -0.041 with landscape-grouped simulation (Fig. 4.21, below). For the abundance models, the average differences were -1.159 for independent field simulation, -1.260 with locallygrouped simulation, and -1.094 with landscape-grouped simulation. 

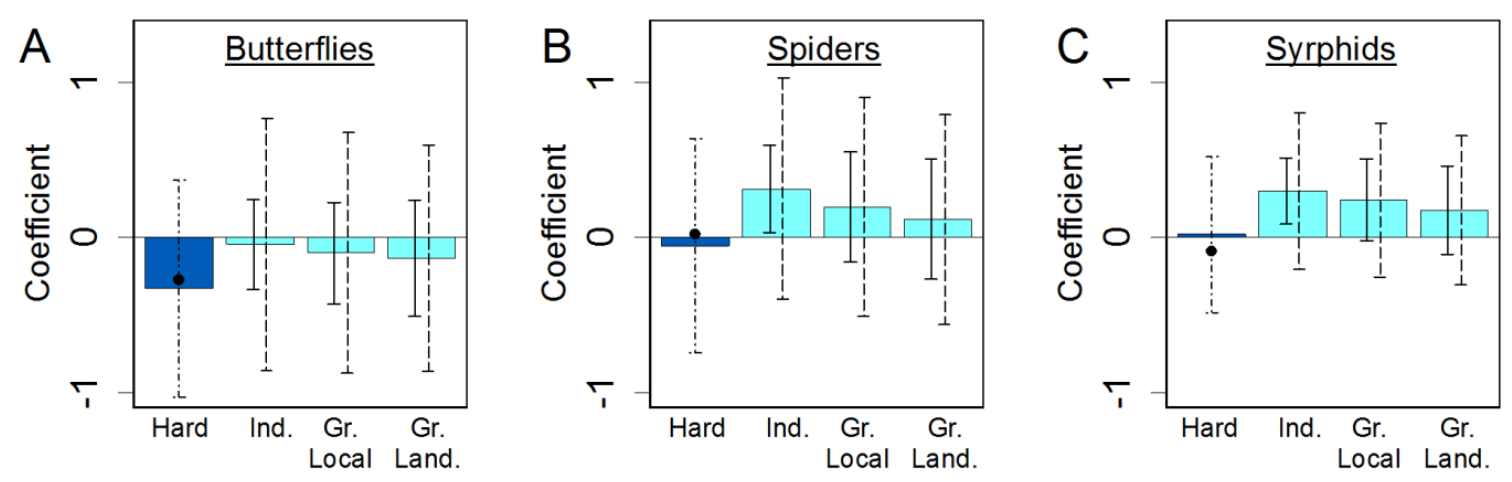

\begin{tabular}{|c|c|c|}
\hline \multicolumn{3}{|l|}{ Legend } \\
\hline \multirow{2}{*}{\multicolumn{2}{|c|}{$\begin{array}{l}\square \text { SHDI coefficient based on hard classification metrics }\left(\hat{\theta}_{\text {hard }}\right. \\
\square \text { Simulated SHDI coefficient }\left(\hat{\theta}_{\text {sim }}\right)\end{array}$}} & $\begin{array}{l}\text { - Coefficient based on } \\
\text { the oriainal dataset }\end{array}$ \\
\hline & & \\
\hline $\begin{array}{l}7.95 \% \text { confidence interval } \\
\text { based on the hard model } \\
\text { averaging standard error } \\
\text { estimate, } \widehat{s e}\left(\hat{\theta}_{\text {hard }}\right)\end{array}$ & $\begin{array}{l}95 \% \text { confidence interval } \\
\text { based on standard } \\
\text { deviation of simulated } \\
\text { coefficients, } \sigma_{\text {sim }}\end{array}$ & $\begin{array}{l}\text { T } 95 \% \text { confidence interval } \\
\text { based on pooled model } \\
\text { averaging standard } \\
\perp \text { error estimates, } \widehat{\operatorname{se}}\left(\hat{\theta}_{\text {sim }}\right)\end{array}$ \\
\hline
\end{tabular}

Figure 4.20 SHDI coefficients based on the hard classification versus those based on independent field (Ind.), locally-grouped (Gr. Local), and landscape-grouped (Gr. Land.) simulation approaches, for the (A) butterflies, (B) spiders, and (C) syrphids gamma diversity models.

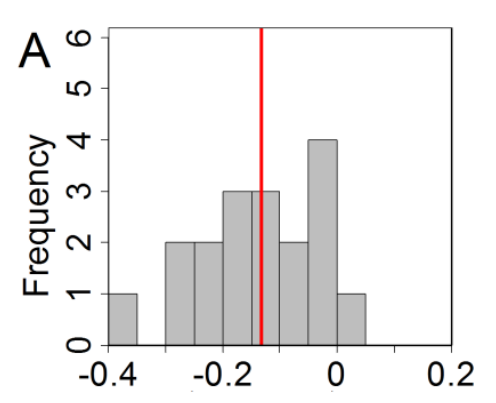

Difference in coefficient

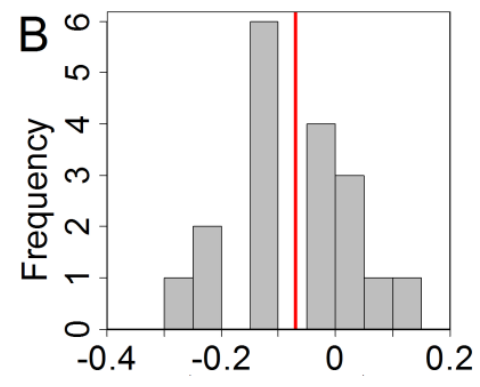

Difference in coefficient

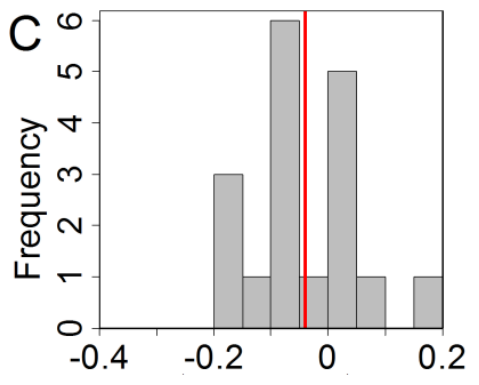

Difference in coefficient

Figure 4.21 Difference (or offset) in SHDI coefficient between those based on the hard classification metrics and the those based on (A) independent field, (B) locally-grouped, and (C) landscape-grouped simulation. Red line represents the mean of the distribution. Coefficients for the abundance models are not included. 
With the decreased offset and increased $\sigma_{\text {sim }}$ under the grouped simulation approaches (Fig. 4.20), the simulated $\widehat{\bar{\beta}}_{\text {SHDI }}$ distributions were more representative of the original $\widehat{\bar{\beta}}_{\text {SHDI }}$ (based on the original, fully ground referenced dataset). Under the grouped simulation approaches, the standard Z-scores for the original $\widehat{\bar{\beta}}_{\text {SHDI }}$ values, relative to the simulated $\widehat{\bar{\beta}}_{\text {SHDI }}$ distributions, more closely approximated a standard normal distribution (across all 23 models) (Fig. 4.22).
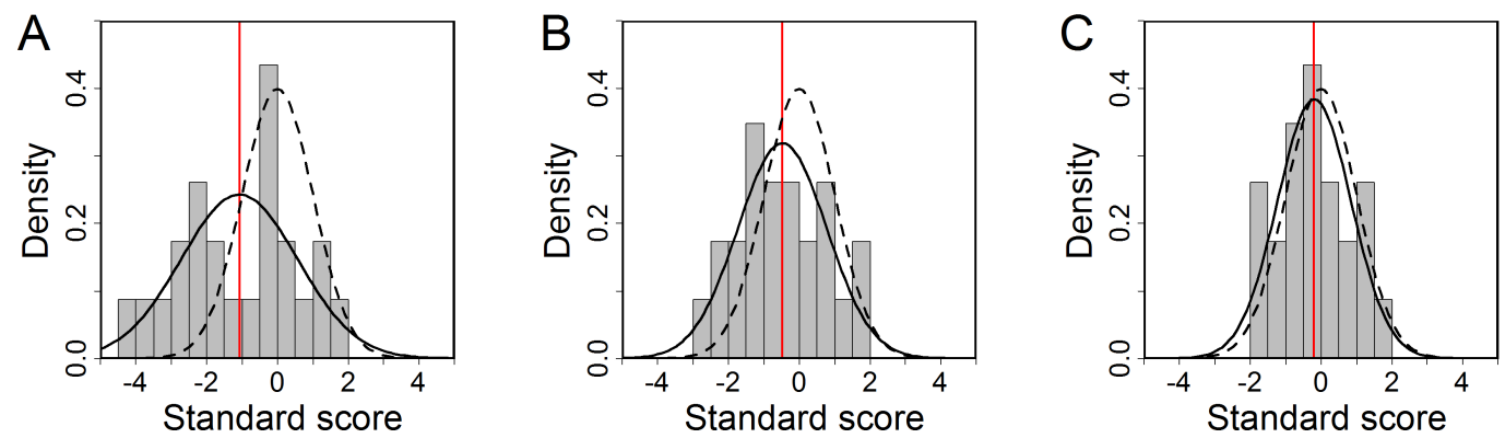

Figure 4.22 Standard Z-scores, across all 23 models, for the original SHDI coefficients (based on original dataset) relative to their corresponding simulated SHDI coefficient distributions under (A) independent field, (B) locally-grouped, and (C) landscape-grouped simulation. The red line represents the mean of the Z-score distribution. The solid curve represents a normal distribution with a mean and standard deviation equal to that of the distribution of standard scores. The dashed curve represents a standard normal distribution. 


\subsubsection{Positional and thematic uncertainties versus the considered uncertainties}

For all models, $\sigma_{\text {sim }}$, representing the uncertainty in $\widehat{\bar{\theta}}_{\text {sim }}$ associated with measurement uncertainty in the landscape metrics (arising from positional and thematic uncertainties in the LC maps), was less than $\widehat{\operatorname{se}}\left(\hat{\bar{\theta}}_{\text {hard }}\right)$ and $\widehat{\operatorname{se}}\left(\hat{\bar{\theta}}_{\text {sim }}\right)$, which represent the uncertainty associated with the sample, given the model, and model selection (Burnham \& Anderson, 2002; Anderson, 2008).

For all models under the independent field simulation approach, the proportion of $\sigma_{\text {sim }}^{2}$ to $\operatorname{var}\left(\hat{\bar{\theta}}_{\text {sim }}\right)$ was higher for $\widehat{\bar{\beta}}_{\text {SHDI }}$, than for $\widehat{\bar{\beta}}_{\text {P.AG }}$ or $\widehat{\bar{\beta}}_{\text {MFS }}$. Between $\widehat{\bar{\beta}}_{\text {P.AG }}$ and $\widehat{\bar{\beta}}_{\mathrm{MFS}}$, the difference in this proportion was less distinct. In some cases it was larger for $\widehat{\bar{\beta}}_{\text {P.AG }}$, and larger for $\widehat{\bar{\beta}}_{\mathrm{MFS}}$ in others. On average, the proportion was 0.022 for $\widehat{\bar{\beta}}_{\mathrm{P} . \mathrm{AG}}$, 0.033 for $\widehat{\bar{\beta}}_{\text {MFS }}$, and 0.148 for $\widehat{\bar{\beta}}_{\text {SHDI }}$ (Table 4.10, below). The model-specific proportions are included in Appendix I.

Under the grouped simulation approaches, the proportion of $\sigma_{\text {sim }}^{2}$ to $\widehat{\operatorname{var}}\left(\hat{\bar{\theta}}_{\text {sim }}\right)$ increased for $\widehat{\bar{\beta}}_{\text {SHDI }}$ while remaining relatively constant for $\widehat{\bar{\beta}}_{\text {P.AG }}$ and $\widehat{\bar{\beta}}_{\text {MFS }}$ (Table 4.10). The proportion, for $\widehat{\bar{\beta}}$ SHDI increased to 0.213 under locally-grouped simulation and 0.280 under landscape-grouped simulation, indicating that the proportion is higher when spatial correlation in the thematic error is taken into account. 
Table 4.10 Proportion of variance in the simulated coefficients $\left(\hat{\bar{\theta}}_{\text {sim }}\right)$ to the simulated unconditional variance estimate from model averaging (pooled across realizations), $\widehat{\operatorname{var}}\left(\hat{\bar{\theta}}_{\text {sim }}\right)$, for the P.AG, MFS, and SHDI coefficients $(\widehat{\bar{\beta}})$ under the independent field and grouped simulation approaches. Values represent the average proportion across all models. The standard deviation of each proportion, across all models, is included in brackets.

\begin{tabular}{cccc}
\hline \multirow{2}{*}{ Simulation approach } & \multicolumn{3}{c}{ Average proportion of $\sigma_{\text {sim }}^{2}$ to $\widehat{\operatorname{var}}\left(\hat{\bar{\theta}}_{\text {sim }}\right)$, for: } \\
\cline { 2 - 4 } & $\hat{\bar{\beta}}_{\text {P.AG }}$ & $\widehat{\bar{\beta}}_{\text {MFS }}$ & $\widehat{\bar{\beta}}_{\text {SHDI }}$ \\
\hline \multirow{2}{*}{ Independent field } & 0.022 & 0.033 & 0.148 \\
& {$[0.012]$} & {$[0.012]$} & {$[0.030]$} \\
Locally-grouped & 0.024 & 0.037 & 0.213 \\
& {$[0.013]$} & {$[0.013]$} & {$[0.059]$} \\
Landscape-grouped & 0.025 & 0.039 & 0.280 \\
& {$[0.013]$} & {$[0.013]$} & {$[0.073]$} \\
\hline
\end{tabular}

Finally, it should be noted that $\widehat{\operatorname{var}}\left(\hat{\bar{\theta}}_{\text {sim }}\right)$ can differ from $\widehat{\operatorname{var}}\left(\hat{\bar{\theta}}_{\text {hard }}\right)$, particularly for $\widehat{\bar{\beta}}_{\text {SHDI }}$. Under the independent field simulation approach, $\widehat{\operatorname{var}}\left(\hat{\bar{\theta}}_{\text {sim }}\right)$ for $\widehat{\bar{\beta}}_{\text {SHDI }}$ was slightly inflated, at $7.02 \%$ larger than the corresponding $\widehat{\operatorname{var}}\left(\hat{\bar{\theta}}_{\text {hard }}\right)$ on average, across all models. This decreased to $4.16 \%$ under locally-grouped simulation. Under landscapegrouped simulation, $\widehat{\operatorname{var}}\left(\hat{\bar{\theta}}_{\text {sim }}\right)$ for $\widehat{\bar{\beta}}_{\text {SHDI }}$ was almost equal to $\widehat{\operatorname{var}}\left(\hat{\bar{\theta}}_{\text {hard }}\right)$, on average, at $0.03 \%$ lower. Examples of these differences can be seen for the $\widehat{\bar{\beta}}_{\text {SHDI }}$ in Figure 4.20. For $\widehat{\bar{\beta}}_{\text {P.AG }}$ and $\widehat{\bar{\beta}}_{\text {MFS }}$ these differences were smaller. The presence of these differences means that the proportions in Table 4.10 would change slightly (on the order of $1 \times 10^{-3}$ ) if $\sigma_{\text {sim }}^{2}$ were to be compared directly to $\widehat{\operatorname{var}}\left(\hat{\bar{\theta}}_{\text {hard }}\right)$ rather than $\widehat{\operatorname{var}}\left(\hat{\bar{\theta}}_{\text {sim }}\right)$.

Looking specifically at $\widehat{\bar{\theta}}_{\text {hard }}$ and the corresponding $\widehat{\operatorname{var}}\left(\hat{\bar{\theta}}_{\text {hard }}\right), 6$ of the $23 \widehat{\bar{\beta}}_{\text {P.AG }}$ and 12 of the $23 \widehat{\bar{\beta}}_{\text {P.AG }}$ were significantly different from zero $(\alpha=0.05)$. None of the 23 
$\widehat{\bar{\beta}}$ SHDI were significantly different from zero. If the $\sigma_{\text {sim }}^{2}$ values from the landscapegrouped simulation approach (Appendix M, Tables M.5 and M.6) were to be taken as being representative of the $\widehat{\bar{\theta}}_{\text {hard }}$ variance associated with positional and thematic uncertainties in the LC maps, and $\widehat{\bar{\theta}}_{\text {hard }}$ standard error estimates were updated accordingly, as in:

$$
\sigma \text { for any given hard coefficient }=\sqrt{\widehat{\operatorname{var}\left(\hat{\bar{\theta}}_{\text {hard }}\right)+\sigma_{\text {sim }}^{2}}}
$$

then the consideration of $\sigma_{\text {sim }}^{2}$ only affected the significance of one of the coefficients, in one of the 23 models. $\widehat{\bar{\beta}}_{\mathrm{MFS}}$ for the beetles abundance model was no longer significant when positional and thematic uncertainties in the LC maps was taken into account.

\subsubsection{Convergence of simulated coefficients and variance estimates}

For the majority of coefficients, the variance estimate associated with measurement uncertainty in the landscape metrics $\left(\sigma_{\text {sim }}^{2}\right)$ had reasonably converged across the 2000 model realizations. Figure 4.23, below, shows the convergence plots for gamma diversity models, under the independent field simulation approach. All plots of the same coefficient ( $\widehat{\bar{\beta}}_{\text {P.AG }}, \widehat{\bar{\beta}}_{\text {MFS }}$, or $\widehat{\bar{\beta}}_{\text {SHDI }}$ ) are on a common y-axis with the exception of the plants models, which tended to have larger coefficients and higher $\sigma_{\text {sim }}^{2}$ in those coefficients.

At the variance scale shown by these plots, the simulations had reasonably converged on a $\sigma_{\text {sim }}^{2}$ estimate, showing little change in variance over the last 500 realizations, relative to the first 500 or 1000 realizations. There were cases, such as those in Figure 4.23 A5 and C3, where slight trends were observed within the last 500 
realizations, suggesting that the $\sigma_{\text {sim }}^{2}$ estimates may have been more precise for certain models. The estimates of $\sigma_{\text {sim }}^{2}$ converged at similar scales for the alpha, beta, and gamma diversity models. The abundance models, which usually had larger coefficients and correspondingly larger $\sigma_{\text {sim }}^{2}$ estimates, tended to converge at a smaller scale (more variation in $\sigma_{\text {sim }}^{2}$ estimates across the last 500 realizations) than the other models. The plots show no major differences in convergence rate between the independent field and grouped simulation approaches (based on visual interpretation). Examples of these convergence plots for the grouped simulation approaches are included in Appendix $\mathbf{J}$.

Examples of $\hat{\bar{\theta}}_{\text {sim }}$ convergence plots for $\hat{\bar{\beta}}_{\text {SHDI }}$ of the syrphids models, across all diversity types and simulation approaches, are shown in Figure 4.24, below. $\hat{\bar{\theta}}_{\text {sim }}$ roughly converged within the first 1000 realizations in most cases (sometimes more realizations, especially for the landscape-grouped simulation approach). Like the $\sigma_{\text {sim }}^{2}$ estimates, $\hat{\bar{\theta}}_{\text {sim }}$ for the alpha, beta, and gamma models converged at a similar scale, while $\hat{\bar{\theta}}_{\text {sim }}$ tended to vary more over the last 500 realizations for the abundance models. Also, like the $\sigma_{\text {sim }}^{2}$ estimates, there were cases where $\hat{\bar{\theta}}_{\text {sim }}$ showed trends within the last 500 realizations, suggesting that these estimates may have been more precise for certain models.

The $\hat{\bar{\theta}}_{\text {sim }}$ convergence shown in Figure 4.24 suggests that the cases of offset between $\hat{\bar{\theta}}_{\text {hard }}$ and $\hat{\bar{\theta}}_{\text {sim }}$ for $\widehat{\bar{\beta}}_{\text {SHDI }}$ (Section 4.2.4.2) were not a result of poor convergence. For example, the offset for $\widehat{\bar{\beta}}_{\text {SHDI }}$ of the syrphids gamma diversity model, under independent field simulation, was 0.280 , whereas the standard deviation in $\hat{\bar{\theta}}_{\text {sim }}$ for the last 500 realizations of its convergence plot (Figure $4.24 \mathrm{C} 1$ ) was only $2.814 \times 10^{-4}$. 

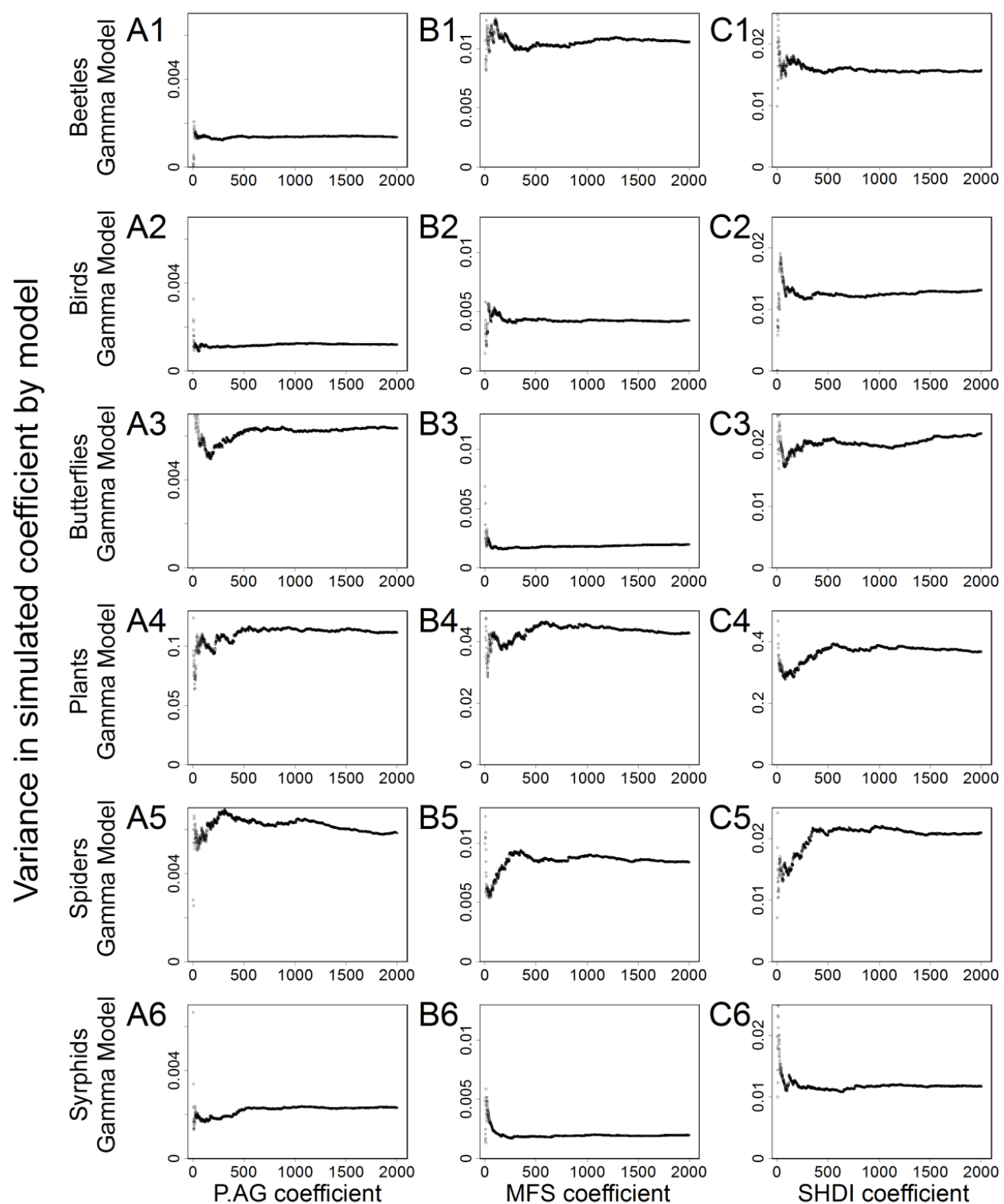

Number of realizations by coefficient

Figure 4.23 Cumulative variance in simulated coefficient $\left(\hat{\bar{\theta}}_{\text {sim }}\right)$ versus the number of realizations used to calculate this variance. Plots represent the degree of convergence of the variance estimates of the simulated coefficients $\left(\sigma_{\text {sim }}^{2}\right)$ for the (A) P.AG, (B) MFS, and (C) SHDI coefficients $(\hat{\bar{\beta}})$ of the (1) beetles, (2) birds, (3) butterflies, (4) plants, (5) spiders, and (6) syrphids gamma diversity models. The values shown are based on the independent field simulation approach. 

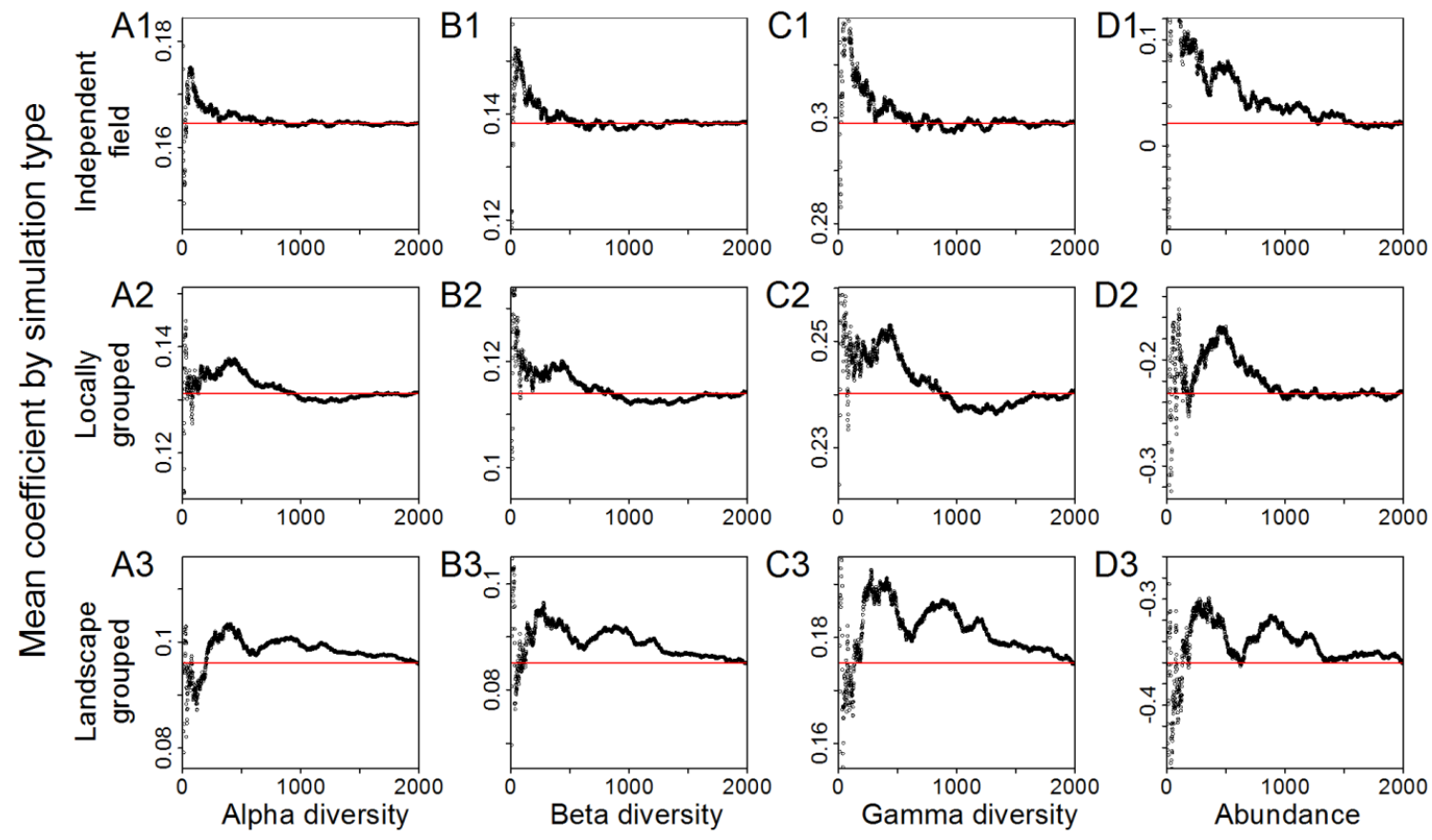

Number of realizations by diversity type

Figure 4.24 Cumulative mean simulated SHDI coefficient ( $\left(\hat{\bar{\theta}}_{\text {sim }}\right.$ for $\left.\widehat{\bar{\beta}}_{\text {SHDI }}\right)$ versus the number of realizations used to calculate this mean. Plots represent the degree of convergence of the simulated coefficients for the syrphids (A) alpha diversity, (B) beta diversity, (C) gamma diversity, and (D) abundance models under the (1) independent field, (2) locally-grouped, and (3) landscape-grouped simulation approaches. The red line represents the mean simulated coefficient, as determined by all 2000 realizations. 


\section{Discussion}

\subsection{SHDI simulation comparison}

This section discusses the results of the limited extent image set classifications and the SHDI simulation comparison (Sections 3.3 and 4.1), in the context of research question 1 (Section 1.3). First the classification accuracy is discussed, as well as ways in which it could be improved (Section 5.1.1). The relationship between $E_{\mathrm{n}}$ and thematic error is briefly discussed in the context of the limited extent classifications (Section 5.1.2), but is expanded on in later sections of the Discussion. The ability of the independent pixel-based, field-based, and homogenous field-based simulation approaches to preserve within-field thematic structure is discussed (Section 5.1.3). Finally, the nature of the simulated SHDI distributions is discussed, in terms of variance and offset from the reference values (Section 5.1.4).

\subsubsection{Classification accuracy}

The accuracy of the hard classifications used for the SHDI simulation comparison ranged from very poor to moderate (Section 4.1.3). While a range of accuracies was desired for the sake of comparison, they leaned towards being too inaccurate for use as a legitimate crop map, particularly the QB classifications. An overall accuracy of at least $85 \%$ is generally desired, with a minimum accuracy of $70 \%$ or $75 \%$ for each individual class (Thomlinson, Bolstad, \& Cohen, 1999; Foody, 2002; De Wit \& Clevers, 2004; Castillejo-Gonzáleza et. al. 2009; McNairn, Champagne, Shang, Holmstrom, \& Reichert, 
2009; AAFC, n.d.). There are several likely reasons for the poor accuracy of these maps, including the classification scheme, training and validation data limitations, the resolution (particularly for the QB imagery), and the classifier (given the nature of the classes).

\subsubsection{Classification scheme}

The classification scheme (Table 3.1) contributed to the low separability (as measured by transformed divergence) of many of the classes in the QB and single date TM images, which likely contributed to lower accuracies. Transformed divergence values less than 1500 , or less than 1700 in some cases, are considered to represent spectrally similar classes, which can lead to accuracy problems (Jensen, 1996; Lillesand et al. 2004; Kavzoglu \& Mather, 2002). In the single date classifications, several class pairs had transformed divergence values less than 1700, particularly amongst the grasslike classes (Appendix C).

Hard classifiers (the posterior probabilities used here were associated with the hard maximum likelihood classifier; Section 3.3.6) operate on the assumption that the image is made up of pure pixels, each containing only one class (Foody, 1996). The grass-like classes (Hay, Pasture, Legumes, and Fallow), however, typically included a mix of similar vegetation types. Hay fields, Legumes fields, and Pasture fields often contained a mix of grass and legume (e.g. alfalfa and clover) vegetation. Also, Fallow fields often contained a lot of grass. Since the spectral characteristics of the crop classes were primarily defined by their vegetation cover, it is not surprising that there was a lot of confusion amongst these classes. 
In cases of high confusion amongst related classes, class aggregation can be used to improve accuracy (Lillesand et. al. 2004). While not included in the results presented here, classifications were performed with merged classes (Hay, Pasture, and Legumes merged, as well as Fallow and Abandoned), resulting in improved accuracies. For example, with the merged classes, the overall accuracies for the per-field classifications of the multi-date TM image set ranged from $85.9 \%$ to $92.9 \%$ across the four landscapes (Appendix F, Tables F.26 to F.29).

\subsubsection{Training and validation data limitations}

The general rule for the amount of training samples for a supervised classification is to have at least 10 to 30 samples for every spectral band (Van Niel, McVicar, \& Datt, 2005; Foody, Mathur, Sanchez-Hernandez, \& Boyd, 2006). Given that the fewest number of training samples used was 222 TM pixels for the Pasture class (perhaps less, as some training samples were removed for any given landscape classification; Section 3.3.4), and since only 4 to 10 bands were used in any of the classifications (the limited extent classifications), it's likely that this rule was met in most classifications. However, the extent from which the training samples could be drawn was limited to that of the QB image. Within this extent, there were a number of Corn and Soybean fields (more than 30 fields) available to draw these samples from, though samples were only drawn from a limited number of Hay (26 fields), Pasture ( 7 fields), Legumes (20 fields), Cereals (9 fields), Fallow (20 fields), and Abandoned (12 fields) fields. Due to the limited number of ground referenced fields available to draw training samples from, the spectral characteristics for a class, across all growth stages and crop health states, may not have 
been fully characterized. This could have contributed to the cases wherein some incorrectly classified fields (atypical, relative to the training data) were assigned a high probability of belonging to the wrong class, as opposed to being assigned to a class with a higher degree of ambiguity (Section 4.1.1).

In addition to limitations surrounding training sample selection, the potential for error in the ground reference set is recognized in the literature (Foody, 2002; Congalton \& Green, 2009). Here, these errors may have reduced the accuracy of the classifications by contributing to confusion between classes (for training data errors) and false errors during accuracy assessment (for validation data errors). Examples of uncertain, and possibly erroneous, ground referenced fields are shown in Appendix G. Boundary interpretation error during digitizing may have also contributed to these errors. Examples of cases wherein more than one land cover may occur within an individual field can be seen in Appendix D.

Errors in the ground referenced data were likely higher in the grass-like classes. When observing from a distance (reference data outside of the LCs), some crop characteristics, such as the proportion of grass to legume vegetation, could be difficult to estimate. The estimation of grass and legume proportions could be further complicated by difficulty in identifying vegetation types in freshly-cut forage crops, and the fact the proportions of grass to legumes can change throughout the growing season, due to weather conditions (OMFRA, 2011c). Some Pasture that had not been grazed yet would appear as Hay or Legumes fields (assuming the electric fence was not visible from the road). Some fields that had been referenced as Fallow (due to a higher proportion of 
weeds) were later observed being cut and bailed. These issues may have contributed to lower accuracies for these classes.

\subsubsection{Resolution and classifier}

For pixel-based classification methods, increased image spatial resolution results in increased within-class spectral variability (Castillejo-González et. al. 2009). In the case of the QB imagery, with only four spectral bands to discriminate between classes and a high spatial resolution relative to the size of the features being mapped (field size), the increased within-class variability likely contributed to the lower classification accuracy. Similar spectral characteristics between classes in the QB imagery can be seen in Appendix B, Figure B.3. van den Berg (2012) performed classifications based on the same dataset, obtaining a much higher overall accuracy when the imagery was resampled to a $30 \mathrm{~m}$ spatial resolution (in addition to a different classification scheme). Myint et al. (2011) performed a pixel-based maximum likelihood classification of QB imagery $(2.4 \mathrm{~m}$ spatial resolution) in an urban area, which included some spectrally similar classes, and compared the results to an object-based classification of the same image. Their pixelbased classification had a relatively low overall accuracy, at 67.6\% (including low accuracies for trees, shrubs, and grasses), but the accuracy increased to $90.4 \%$ with the object-based classification approach.

On the other hand, the lower spatial resolution of the TM imagery appeared to produce its own accuracy issues, particularly near the boundaries of small fields or fields with a high perimeter-area ratio. In the TM classifications (Figs. 4.1 and 4.2), cases can be seen where fields had fairly high probabilities of class membership in the correct class 
towards the center of the field, with low probabilities in this class near the field boundary. This was likely caused by mixed pixels near the boundary, and probably affected smaller fields (or other fields that have a higher perimeter-area ratio) more than larger fields, assuming that there were no major differences in shape. Smith et al. (2002) studied the relationship between patch size and classification accuracy in TM-based thematic maps across much of the eastern United States, including some agricultural classes. They found a significantly negative relationship between the patch size and classification accuracy.

Despite these issues, the resolution was not changed for any of the image sets. The contrast that the QB imagery provided relative to the TM imagery resolution, in terms of the number of objects that were free to vary with a given field, provided an opportunity for comparison.

The use of the Bayesian classifier, which assumes that training data are normally distributed in any given band (Lillesand et al. 2004), also likely contributed to lower accuracies. While some classes, such as Corn and Soybean, had fairly normally distributed training data, classes such as Hay, Pasture, and Legumes, which are typically cut or grazed several times throughout the growing season (OMFRA, 2011c, 2012c, 2012d), were more prone to skewness and multimodality (Appendix B).

\subsubsection{Entropy}

Despite the generally poor accuracy of the hard classifications, the range of $E_{\mathrm{n}}$ values available for comparison was quite reasonable, ranging from as low as 0.16 to as 
high as 0.70 , on average, at the landscape scale (Section 4.1.2; Table 4.1). The differences in $E_{\mathrm{n}}$ values between the high and low accuracy image set classifications, and between the correctly and incorrectly classified fields within these classifications, suggest an inverse relationship between classification accuracy and $E_{\mathrm{n}}$ (Appendix F, Table F.1, Figure F.1).

Class membership probabilities from the classifier are representative of cases where a class was assigned with high or low certainty, or ambiguity in the case of $E_{\mathrm{n}}$ (Gonçalves et al. 2012), and can be used as a measure of classification quality (Foody, 1996). However, these probabilities are dependent on how the classifier is trained (Gonçalves et al. 2012). Cases where fields had been incorrectly classified despite low $E_{\mathrm{n}}$ (Appendix F, Figure F.1) support the idea that the training set drawn from the limited extent was not representative of all crop states. Additionally, while hard training data were used for these classifications, similar vegetation types in training samples of the grass-like classes likely contributed to higher $E_{\mathrm{n}}$ in those classes, even for correctly classified fields (Table 4.2, Appendix F, Figure F.1). The non-fully-representative training data and the spectrally similar grass-like classes could have somewhat diminished the relationship between $E_{\mathrm{n}}$ and classification quality.

\subsubsection{Structure preservation}

Similar to the findings of Canters (1997), the field-based simulation approach preserved within-field structure better than the independent pixel-based simulation approach (Section 4.1.4). However, in terms of structure preservation, the contrast 
between the independent pixel-based and field-based simulation approaches was larger for the higher $E_{\mathrm{n}}$, lower accuracy classifications (i.e. single date $\mathrm{TM}$ and QB classifications). This was likely due to their greater potential for the deterioration of within-field structure under the independent pixel-based approach, relative to the lower $E_{\mathrm{n}}$, higher accuracy classifications (i.e. multi-date TM classifications). For example, under independent pixel-based simulation of high $E_{\mathrm{n}}$ pixels within a given field, independent PRNs drawn for each pixel makes it likely that some of those pixels will take on an alternate class (as opposed to the most-likely) in any given realization, and in a somewhat random pattern due to the spatially independent PRNs. However, if the pixels within the field have a high probability of belonging to a particular class (lower $E_{\mathrm{n}}$ and likely higher accuracy), less are going to be assigned to an alternate class in a given realization, resulting in less structure deterioration. This is seen in the realization examples provided in Section 4.1.4 (southernmost Corn field, marked '1'), and the effects on the SHDI distributions can be seen in Figure 4.7.

Deterioration of within-field structure can still occur under the field-based simulation approach. Under this approach, pixels which are assumed to vary together can still be assigned to separate classes within an individual realization, due to differences in their PDFs (Canters, 1997). This can be quite clearly seen in the realizations of the QB classification (Fig. 4.6), and near field boundaries in the TM classifications (Figs. 4.4 and 4.5). Clusters of pixels of different classes appear within fields in the QB realizations, likely due to the sensitivity of these classifications to crop condition (growth stage and health) and noise. For cases in the TM classifications, this likely occurs due to mixed 
pixels near field boundaries having different PDF characteristics, or larger scale variations in crop condition.

With the homogenous field-based simulation approach, there was perfect structure preservation within fields, assuming that the fields were actually thematically homogeneous. Since the boundaries of fields were known with reasonable certainty in most cases, this assumption was reasonable; though it was not without issues. First, while not a major issue with regards to calculating SHDI, aggregating the class membership probabilities to the field level resulted in some information loss, in terms of the distribution of classification confidence within fields. Second, mixed pixels, small portions of non-cropped area within fields, or portions of highly atypical crop condition within fields can influence the PDFs of the full field. Given enough realizations, confusion in those problematic portions will result in a realization wherein the full field is assigned to an alternate class (e.g. a Corn field being fully represented as Fallow in a given realization, due to mixed pixels existing only along its boundary). These portions also likely contributed to higher per-field mean $E_{\mathrm{n}}$ values, relative to the per-pixel mean

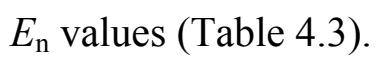

\subsubsection{Simulated SHDI distributions}

Here, the characteristics of the simulated SHDI distributions (Section 4.1.5) are discussed with respect to their variance (Section 5.1.4.1) and offset, or bias, from either the reference SHDI values (from the ground referenced, trimmed field boundaries) or the hard classification SHDI values (Section 5.1.4.2). 


\subsubsection{SHDI distribution variance}

The larger variance in the simulated SHDI distributions from the field-based simulation approach, relative to those from the independent pixel-based approach (Section 4.1.5), is similar to the findings of Canters (1997). Canters (1997) had found increasing variance in the simulated metrics as the degree of pixel grouping increased. The more constraints on the freedom with which pixels can vary individually within a field, the higher the variance tends to be for metrics based on both area and thematic class (e.g. SHDI or areal coverage of an individual class), across a given set of realizations. Therefore, it was not surprising that the simulated SHDI distributions based on the homogenous field-based simulation approach, where there was no freedom for withinfield variability, exhibited the largest variances, while those based on the independent pixel-based approach exhibited the smallest.

Under the field-based and homogenous field-based simulation approaches, the classifications with larger overall $E_{\mathrm{n}}$ exhibited larger variances in their SHDI distributions, as expected (Section 4.1.5). However, under the independent pixel-based approach, the expected positive relationship between variance and entropy did not fully hold. It held for the TM classifications, with larger variances in SHDI distributions corresponding to the single date TM classifications, relative to the multi-date TM classifications (Table 4.4) which, on average, had lower per-pixel $E_{\mathrm{n}}$ (Table 4.1). However, the QB classifications which, on average, had the highest overall per-pixel $E_{\mathrm{n}}$, also had, by far, the lowest variances in SHDI distributions (Table 4.4). This can, again, be attributed to the freedom to vary, but in terms of image resolution (the QB imagery 
had a much smaller pixel size than the TM imagery) rather than simulation approach. The cost, in terms of variance estimation, of failing to account for spatially correlated thematic error when generating map realizations, appears to be higher for cases where the minimal mapping unit is smaller, relative to spatial extent of that correlation.

\subsubsection{SHDI offset}

There were a couple of offset issues observed in Section 4.1.5. First, the simulated SHDI distributions were offset from the reference SHDI values. Second, the simulated SHDI distributions were offset from the SHDI values based on the hard classifications.

The offsets between the medians of the simulated SHDI distributions and the reference SHDI values appeared to roughly, and inversely, correspond with classification accuracy (Table 4.3; Fig. 4.7). Distributions based on the very low accuracy QB classifications were the furthest offset, while those based on the multi-date TM classifications tended to be the least offset. Additionally, distributions from the homogenous field-based approach, which was based on the higher-accuracy, per-field classifications, tended to be less offset than the corresponding distributions from the independent pixel-based or field-based approaches, which were based on the loweraccuracy per-pixel classifications. However, there were exceptions when comparing this relationship between landscapes. For example, the distributions based on the QB classification of Landscape D were amongst the furthest offset, despite the fact that Landscape D had the highest accuracy QB classifications. It is clear that accuracy, alone, 
cannot fully explain the degree of offset. Other factors, such as the classes adopted for incorrectly classified fields, likely also influence the offset.

Also surprising was the tendency for the median of the simulated SHDI distributions, as well as the hard classification SHDI values, to be consistently offset towards greater diversity, relative to the reference values. This was likely associated with low spectral separabilities amongst some classes, particularly for the QB classifications.

The consistent offset between the medians of the simulated distributions and the hard classification SHDI values, which were shifted towards greater diversity (even under homogenous field-based simulation), highlights an issue with generating realizations for landscapes where there is spatially correlated error between fields (as opposed to pixels within a field). Just as there was a need to account for spatially correlated error when sensitizing pixels within a field (Canters, 1997), there can also be a need for accounting for spatial correlated thematic error when sensitizing similar, nearby fields within a landscape. As failure to account for within-field spatial autocorrelation can lead to a deterioration of within-field structure in realizations (Canters, 1997), failure to account for between-field spatial autocorrelation can lead to a deterioration of landscape structure (in terms of crop type). Between-field spatial autocorrelation was not considered when generating realizations based on the limited extent classifications, but was for some of the simulation approaches (locally-grouped and landscape-grouped simulation) based on the broad, year-2011 classifications of Sections 3.4 and 4.2. The influence of these simulation approaches are discussed in following sections. 


\subsection{Effects of positional and thematic uncertainties on the biodiversity models}

This section discusses the results of the analysis of the effect of positional and thematic uncertainties on the biodiversity models (Sections 3.4 and 4.2 ) in the context of research questions 2 and 3 (Section 1.3). The results of the year-2011 image classification are first discussed in terms of hard accuracy, entropy, and the class membership probabilities (Section 5.2.1). The positional uncertainty estimation and simulation results are then discussed in terms of the vertex positioning and boundary interpretation (Section 5.2.2). The results of the simulated landscape metrics (Section 5.2.3) and corresponding simulated model averaged coefficients (Section 5.2.4) are then discussed.

\subsubsection{Image classification and thematic uncertainty}

The characteristics of the year-2011 TM image classification used to assign crop types to fields for the purpose of calculating SHDI (Sections 3.4.3 and 4.2.1) will be discussed here, including the classification accuracy (Section 5.2.1.1) and $E_{\mathrm{n}}$ of the class membership probabilities (Section 5.2.1.2).

\subsubsection{Classification accuracy}

The accuracy of the hardened classification was moderate, at $83.4 \%$ (Section 4.2.1.1), just shy of the common $85 \%$ overall accuracy goal (Section 5.1.1). Additionally, some classes, particularly Legumes and Fallow, had accuracies well below $70 \%$. While having some problematic classes that do not meet the class-specific 
accuracy targets is not unheard of (De Wit \& Clevers, 2004), often these classes are aggregated or merged (Thomlinson et al. 1999; McNairn et al. 2009). For this classification, classes such as Hay, Pasture, and Legumes (possibly even Fallow) were similar enough that they could have been merged, but were kept separate for compatibility with the models (as was the case for the limited extent classifications; Section 3.3.6). Aside from the merging of classes, accuracy could likely have been improved by addressing some of the issues discussed in Section 5.1.1. Atmospheric correction and further scrutiny of training samples could have been particularly helpful.

While atmospheric correction may not be necessary when attempting to solely classify pixels or objects (no comparison of spectral properties between dates, other than to distinguish between classes) based on single date imagery or composite multi-date imagery (Song, Woodcock, Seto, Lenney, \& Macomber, 2001; De Wit \& Clevers, 2004), correction for spatially varying haze within an individual image, which was quite apparent in the 5 July TM image (Fig. 3.15), could have improved accuracy. This could have involved identifying regions of the image that were clear versus hazy (e.g. via Tasselled Cap transformation) and performing histogram matching between them (Richter, 1996; Liang, Fang, \& Chen, 2001).

As discussed in Section 5.1.1.2, there was some uncertainty associated with the collection of reference data outside of the LCs, particularly for the grass-like classes. Additionally, training samples (pixels) were drawn from training areas that were assumed to be thematically homogenous, but may have occasionally included samples that were highly atypical of their class, due to fact that crop condition is not always homogeneous throughout a field. Gonçalves et al. (2012) were able to increase the accuracy of a 
problematic class (a 'non-vegetated area' class, as determined by a fuzzy minimumdistance-to-means classifier) by removing training samples that exhibited high uncertainty measures (like $\left.E_{\mathrm{n}}\right)$ in preliminary classifications. Scrutiny and possible removal of training pixels that exhibited higher $E_{\mathrm{n}}$ values could have improved the accuracy of the classifications performed in this study.

Finally, it should again be noted that the classification scheme was not fully exhaustive. Supervised image classification typically assumes that pixels or objects are assigned to one class of an exhaustively defined set (Foody, 2002). For these classifications, validation was performed only for classes that were included in the classification scheme, meaning that the true accuracy may have been slightly lower than reported. However, the seven considered classes did account for $95.9 \%$ of cropped area in the year-2011 LCs, with $0.8 \%$ of the cropped area being known to belong to unconsidered classes such as Mixed vegetables, Peas, Strawberry, or Apple (Table 3.1). The remaining $3.4 \%$ of the cropped area within these LCs consisted of unknown or unreferenced crop types.

\subsubsection{Entropy and class probability estimates}

$E_{\mathrm{n}}$ in the broad classification differed between classes in a similar manner to $E_{\mathrm{n}}$ in the limited classifications (discussed in Section 5.1.2), likely due to similar reasons.

The tendency for $E_{\mathrm{n}}$, or class assignment ambiguity, to be more associated with error for some classes (Section 4.2.1.2) highlights an issue regarding how often incorrectly classified fields might adopt an alternate class across realizations, relative to correctly classified fields. For example, average $E_{\mathrm{n}}$ for incorrectly classified Corn fields 
was about $102 \%$ greater than that of correctly classified Corn fields (Table 4.8), whereas $E_{\mathrm{n}}$ for incorrectly classified Hay fields was only about $21 \%$ greater than that of correctly classified Hay fields. Similarly, Corn fields had a mean (area weighted) maximum class membership probability of $85.2 \%$ for correctly classified fields and $57.0 \%$ for incorrectly classified fields, whereas Hay fields had a mean (area weighted) maximum probability of $63.3 \%$ for correctly classified fields and $53.3 \%$ for incorrectly classified fields. Hay fields, a class which had lower class-specific accuracies than Corn, would tend to more frequently adopt an alternate thematic class across realizations than Corn fields, as expected. However, Corn fields would tend have a larger difference in this frequency between correctly and incorrectly classified fields, compared to Hay fields.

The difference seen in the preceding example is likely associated with the fact that many of the training samples for the grass-like classes contained mixes of similar vegetation types (discussed in Section 5.1.2). Foody (1996) suggests that, in such cases, accuracy measures based comparison with validation data (e.g. those based on the error matrix) may be a more appropriate measure of classification quality. However, the scenario adopted by this thesis involved assuming that no ground reference data was available (for classification and simulation purposes; still used for validation) within the year-2011 LCs. This would have made landscape-level accuracy estimates difficult. Possible alternative measurements of classification quality are discussed later, in Section 5.4.1.

Despite the issues surrounding the relationship between the $E_{\mathrm{n}}$ of the class membership probabilities and error, incorrectly classified fields usually had a correct second or third most-likely class (Section 4.2.1.3). If the probabilities of these second or 
third most-likely classes were comparable in magnitude to the most-likely class (incorrectly classified fields tended to have higher $E_{\mathrm{n}}$ ), an incorrectly classified field would likely have adopted the reference class several times across the 2000 realizations.

\subsubsection{Positional uncertainty}

The characteristics of the positional uncertainty estimates, used for sensitizing field boundaries (Sections 3.4.1, 3.4.2, and 4.2.2), will be discussed here, including the vertex position uncertainty estimates (Section 5.2.2.1) and the boundary interpretation uncertainty estimates (Section 5.2.2.2).

\subsubsection{Vertex position uncertainty estimates}

As described in Section 3.4.1, re-digitizing and vertex matching was performed to establish uncertainty estimates, represented by normal distributions. Vertex matching (or point matching) is a legitimate approach to quantifying positional uncertainty when the features have a fair amount of distinct corners (Goodchild \& Hunter, 1997). While representing this uncertainty through probabilistic estimates based on vertex matching may be less sensitive to outliers than the traditional (deterministic) epsilon band representation (Goodchild \& Hunter, 1997; Section 2.4.2), this approach may have issues surrounding the fact that uncertainty is being measured only at distinct points within features (e.g. near perpendicular corners), and may not be representative of all vertices of these features (e.g. vertices placed along curved boundaries) (Goodchild \& Hunter, 1997). Accounting for potential bias towards vertex position uncertainty at field corners, as opposed to vertices places along a boundary feature (e.g. to maintain the shape of a 
slight curve) could have provided better uncertainty estimates, though it would likely not have been as simple as vertex matching at field corners.

In addition to issues associated with vertex matching, the approach of sensitizing vertices independently (Section 3.4.4.1) may not be representative of the true nature of the positional error in these vertices. Tong et al. (2013) proposed the statistical simulation based error model wherein correlation was not just considered for endpoints of a line segment, but for all vertices in a polyline or polygon (via a variance-covariance matrix), producing error bands similar to those of Shi \& Liu's (2000) G-band error model (Section 2.4.2). Correlated error (spatial or temporal) between vertices can be considered by the Data Uncertainty Engine (Brown \& Heuvelink, 2007; de Bruin, Heuvelink, \& Brown, 2008), but was not considered in this study (Section 3.4.4.1). Because positional error was only measured for a small subset of all vertices, at distinct corners (not representative of all distances between vertices), establishing a semivariogram would have been problematic. Additionally, the large number of vertices would likely have severely slowed the generation of spatially correlated vertex realizations (over 30,000 vertices sensitized 2000 times in both the North-South and EastWest directions).

The assumption of positional error following a normal distribution is recognized in the literature (Shi et al.1999; Shi \& Liu, 2000; Zhang \& Goodchild, 2002; Tong et al.2013), and deviations from this distribution in the tails, as occurred in this case (Fig. 3.12), is not unheard of (Goodchild \& Hunter, 1997). In this case, the deviations from normality may have been, in part, due to the fact that the offset between both the year2008 and year-2011 re-digitized vertices and the reference vertices were considered for 
several of the matching cases (where it was not fairly clear which image the reference vertex was based on). Since, in these cases, there was slight error associated with the alignment of the images themselves, the offset values may have been slightly inflated.

It is quite clear that there are several ways in which the positional uncertainty estimates in this study could have been improved, potentially improving the relationship between the variance in area of the simulated versus re-digitized field boundaries (Fig. 4.13). However, with the simple approach taken in this study, the relationship was roughly represented.

\subsubsection{Boundary interpretation uncertainty estimates}

The decision to consider boundary interpretation uncertainty was based on discussion by Edwards \& Lowell (1996) regarding global and local error estimates, and the fact that local context can have a large influence on error. While their study involved a different environment, the same could be said about the environment of this study. The fairly random error associated with vertex positioning, present in all field boundary representations, could be represented with a global error estimate. The local boundary interpretation uncertainty was only considerably present in a minority of fields but, for those fields, it likely had a much larger influence on the variance in area than the global uncertainty associated with vertex positioning.

The approach of this study (Section 3.4.2) involved a single interpreter identifying cases of boundary interpretation uncertainty and assigning probability estimates based heavily on visibility estimates. Ideally, the identification of cases and probability estimates would have been based on the judgments of several interpreters. This, 
however, would have been much more resource intensive, given the number of fields within the dataset.

The use of visibility estimates based on the edge detection likely provided more consistent probability estimates than if they been assigned by a single interpreter, but the approach still had problems. These included the rather subjective selection of edge detector thresholds, the lack of validation, slight brightness differences within and between landscapes, and the edge detector's inability to take colour and other contextual information into consideration.

The detector thresholds were selected through a trial and error procedure such that the three threshold ranges would detect what a human interpreter considered low, medium, and highly visible edges (Section 3.4.2.1). This approach to threshold selection was not very quantitative, and the thresholds were matched to subjective estimates of edge visibility. Additionally, since there were no reference probability estimates (e.g. estimates based on split or merge frequency across judgments from several human interpreters) available for comparison, validation of these estimates was not performed.

There was an issue with slight changes in brightness across each photo, likely associated with bidirectional reflectance (Lillesand et al. 2004; Tuominen \& Pekkarinen, 2004). Since the LCs only accounted for a small to moderate portion of each photo's field of view (each photo covered an area of roughly $2.7 \mathrm{~km}$ by $3.5 \mathrm{~km}$ ), the change in brightness within each LC appeared to be fairly minor (visual interpretation). However, since each LC did not lie in the same relative location within each photo's extent (different viewing angle), and since the illumination conditions may have changed with acquisition time, the photo brightness between LCs may have differed. Local radiometric 
correction using satellite imagery (like TM imagery) can be performed without determining the bidirectional reflectance function of the land cover types (Tuominen \& Pekkarinen, 2004), but the influence that this would have on edge detection is unclear.

There was an issue regarding the fact that the Canny edge detector primarily considered changes in brightness, not accounting for all factors that a human interpreter would consider (e.g. colour, texture, and location relative to nearby features.). Therefore, the relative influence of very bright features (e.g. exposed soil) and very dark features (e.g. heavy shadow) often had more of an influence on the split or merge probabilities than desired. While leeway for interpretation was provided to adjust for these limitations (Section 3.4.2.1), the established probability estimates were still very rough estimates of how those cases might be interpreted across several human perspectives.

Finally, the boundary alternates that involved the addition or removal of entire fields from consideration (Section 3.4.2.2), which were based on interpretation alone, were also very rough, subjective estimates. These decisions were based on characteristics of the entire field (e.g. colour and texture suggesting the presence of woody vegetation, proximity to buildings, etc.) rather than potential edges, so edge detection was of little use for these cases. Obtaining probability estimates based on TM imagery classification would have been difficult, due to the presence of common vegetation types (e.g. between Fallow versus Abandoned, or Hay versus residential lawn), and the tendency of these uncertain fields to be small (higher proportion of mixed pixels). Like the merge and split cases, the estimates could have been improved based on the frequency of inclusion or exclusion by multiple human interpreters. 
Despite the rough probability estimates, by considering this local boundary uncertainty, contextual factors influencing the field boundaries, which couldn't reliably be represented globally, were still able to be represented (e.g. the circled area in Fig 4.11). The frequency with which alternates occur across realizations, however, is likely biased towards the viewpoint of the single interpreter.

\subsubsection{Simulated landscape metrics}

The simulated variance within the P.AG and MFS metrics of individual LCs was low, relative to the variance between LCs (Section 4.2.3.1). Unlike the P.AG and MFS metrics, which were not influenced by thematic uncertainty associated with the image classification, the SHDI metrics were influenced by all forms of uncertainty that were considered. Compared to P.AG and MFS, the SHDI metrics showed higher simulated variance within the LCs relative to the between LC variance. These findings are not terribly surprising, given that the digitizing was based on high resolution air photos from multiple dates (many of the year-2008 edges were still unchanged in the year-2011 and year-2012 air photos), with fairly clear edges in most cases. The image classifications, on

the other hand, had less than ideal imagery coverage (poor coverage in July, with no decent coverage in August and September), a classification scheme that included several spectrally similar classes, and other issues discussed earlier (Section 5.2.1).

Offsets between the hard metrics and the mean of the simulated metrics were clearly visible in some cases (Figs. 4.14, 4.15, and 4.16). These offsets occurred in certain cases for all three metrics. 
Offsets for the P.AG metrics tended to occur in LCs that contained alternate field boundaries involving the addition or removal of fields, or significant portions of fields, from consideration (Fig. 3.13; Section 3.4.2.2). The vertex position simulation, alone, did not appear to produce the offset.

Offsets in the MFS metrics also tended to occur in cases where there were alternate field boundaries, but were not limited to those cases where fields were being removed or entered into consideration. Cases where there was an imbalance in the nature of potential splits or merges (Fig. 3.13; Section 3.4.2.1) likely also influenced these offsets. For example, for a LC with four potential splits, at $50 \%$ probability each, and no potential merges, only about $6 \%$ of the realizations would be represented by the reference boundaries, with the rest containing splits and a reduced MFS metric, relative to the hard metric.

Unlike the P.AG and MFS metrics, which had offset cases but little consistency in offset direction, SHDI metric offsets, under the independent field simulation approach, were more consistent (Fig. 4.16; see Appendix K for histograms of metric offsets). For the majority of the year-2011 SHDI distributions, there was a visible offset wherein the mean of the simulated distribution was larger than the hard metric (sometimes more subtle, sometimes very clear). This bias towards inflated SHDI in the simulated metrics is likely due, in part, to spatial autocorrelation between fields not being taken into consideration under this simulation approach. LCs that exhibited larger offsets tended to be dominated by one or two crop types, despite the presence of many fields (Section 4.2.3.2; Fig. 4.17), suggesting that there was some degree of spatial autocorrelation in crop types between fields (Moran's I statistics for per-field probabilities of class 
membership are included in Appendix L). Similar to the deterioration in within-field structure under the independent pixel-based simulation approach (Section 5.1.3), under the independent field simulation approach, structure in thematic class between spatially correlated fields likely deteriorated as well (discussed in Section 5.1.4.2).

The grouped simulation approaches tended to reduce the bias towards higher SHDI in the simulated metrics (Section 4.2.3.2). However, differences between LCs in terms of the effectiveness of these approaches highlights a couple of issues. First, since SHDI was based on both field class and field area, it's likely that the alternate field boundaries also influenced the SHDI offset for some LCs. For example, LC 100, which showed less offset reduction under the grouped simulation approaches relative to the other LCs that were shown in Figure 4.18, had also shown visible offset in the simulated P.AG and MFS metrics due to alternate boundary representations. Second, the fieldbased simulation approach was proposed to take within-field spatial autocorrelation into account (Canters, 1997), where it may be reasonable to assume that the grouped units (pixels) are fairly homogenous, and errors are highly correlated. However, when the units are fields within a LC, this assumption can be more difficult to justify, as they may be less homogenous in terms of their characteristics (more potential for differences in terms of growth stage, crop health, etc.).

\subsubsection{Model averaged coefficients}

Two forms of offset observed in the model averaged coefficients are discussed in this section. First, offsets between the coefficients that were based on the original dataset 
(ground referenced for both years) and those based on the hard dataset ( $\left.\hat{\bar{\theta}}_{\text {hard }}\right)$ are discussed (Section 5.2.4.1). Second, the offset between the coefficients that were based on the hard dataset $\left(\widehat{\bar{\Theta}}_{\text {hard }}\right)$ and those based on the mean of the coefficient realizations $\left(\hat{\bar{\theta}}_{\text {sim }}\right)$ are discussed (Section 5.2.4.2).

\subsubsection{Coefficient offset between original and hard coefficients}

The offsets between the original coefficients and $\widehat{\bar{\theta}}_{\text {hard }}$ (Section 4.2.4.1) represent the error associated with modelling with year-2011 crop types based on image classification rather than ground referencing. Since the field boundaries of the hard dataset were the same as those of the original dataset (P.AG and MFS metrics unchanged), it's not surprising that the offsets were primarily in $\widehat{\bar{\beta}}_{\text {SHDI }}$. Some small offsets for $\widehat{\bar{\beta}}_{\text {P.AG }}$ and $\widehat{\bar{\beta}}_{\text {MFS }}$ were likely due to the $\widehat{\bar{\beta}}_{\text {SHDI }}$ offsets (slight interaction between predictor variables).

The $\widehat{\widehat{\beta}}_{\text {SHDI }}$ offsets (original versus hard) were larger for some models, suggesting that the coefficients for some models may be more sensitive to the error associated with image classification. This sensitivity may be related to the fact that, for some models, the relationships were better established by data from one of the two years, as indicated by the year indicator variable (Section 2.6.1). Many of the models that showed larger SHDI coefficient offsets, also had larger, negative year indicators (Appendix M), suggesting that the relationships were more influenced by the year-2011 dataset, for which the hard dataset likely contained greater thematic error (compared to the ground referenced year- 
2012 maps). However, not all of those models that had negative year indicators showed large SHDI offsets.

\subsubsection{Coefficient offset between hard and simulated coefficients}

Since this study adopted the scenario wherein the year-2011 crop types were based on image classification, offsets between the original coefficients and $\widehat{\bar{\theta}}_{\text {hard }}$ were somewhat expected, given that image classification is generally more susceptible to thematic error than ground referencing. Therefore, these offsets were focused on less than the offsets between $\widehat{\bar{\Theta}}_{\text {hard }}$ and $\widehat{\bar{\Theta}}_{\text {sim }}$, which were less expected. The simulations were an attempt to quantify uncertainties associated with the hard dataset. However, when $\widehat{\bar{\theta}}_{\text {hard }}$ lies in the extreme tail of the simulated distribution, outside of the $95 \%$ confidence intervals in some cases, it is hard to make the case that the variance in the simulated distribution is representative of uncertainty associated with $\widehat{\bar{\theta}}_{\text {hard }}$.

Cases of offset ( $\widehat{\bar{\Theta}}_{\text {hard }}$ versus $\widehat{\bar{\Theta}}_{\text {sim }}$ ) tended to be largest for $\widehat{\bar{\beta}}$ SHDI (Section 4.2.4.2). Similar to the offset in the SHDI metrics (Section 5.2.3), the large $\widehat{\bar{\beta}}_{\text {SHDI }}$ offsets tended to be directional, with $\widehat{\bar{\theta}}_{\text {sim }}$ values being larger than $\widehat{\bar{\theta}}_{\text {hard }}$ (not in terms of magnitude, but a shift towards more positive values). $\widehat{\bar{\beta}}_{\text {P.AG }}$ and $\widehat{\bar{\beta}}_{\text {MFS }}$ coefficient offsets were present, but generally not as large as the $\widehat{\bar{\beta}}_{\text {SHDI }}$ offsets, and less consistent in terms of offset direction. These similarities to the metric offsets (Section 5.2.3) suggest that the coefficient offsets arose due to those metric offsets. Additionally, just as the grouped simulation approaches reduced offset in the SHDI metrics (Fig. 4.18), the approach also 
reduced the offset in $\widehat{\bar{\beta}}$ SHDI of individual models (Fig. 4.20) and reduced the overall the bias towards more positive simulated $\widehat{\bar{\beta}}_{\text {SHDI }}$ (Fig. 4.21).

Given that the landscape grouped simulation approach reduced the bias associated with $\widehat{\bar{\beta}}_{\text {SHDI }}$ the most (Section 4.2.4.2), there is a temptation to take the $\sigma_{\text {sim }}^{2}$ estimates from this approach as being the most representative of the true variance. However, it may be a bit extreme to assume that, regardless of their relative position within a LC, fields of the same hard class can have highly correlated thematic error. Therefore, no single simulated variance comparison result was selected as being most representative. The results for all three simulation approaches were presented, with the assumption that the true influence of the positional and thematic uncertainties, with respect to $\widehat{\bar{\beta}}$ SHDI, lay somewhere between the independent field and landscape grouped extremes (Section 4.2.4.3). However, the true value may lie more towards those results of the grouped simulation approaches, given the reduced bias.

\subsubsection{Variance comparison}

The variance in the model coefficients associated with measurement uncertainty in the landscape metrics (arising from positional and thematic uncertainties in the LC maps), was fairly low, relative to the variance associated with the sample and model selection (Section 4.2.4.3). The low proportions of $\sigma_{\text {sim }}^{2}$ to $\widehat{\operatorname{var}}\left(\hat{\bar{\theta}}_{\text {sim }}\right)$ for $\widehat{\bar{\beta}}_{\mathrm{P} . \mathrm{AG}}$ and $\widehat{\bar{\beta}}_{\mathrm{MFS}}$ across all simulation approaches (Table 4.10) suggests that the assumption of error-free independent variables by the original models was not unreasonable. However, if the LC maps were to be based mainly on digitizing and image classification alone, or if the 
existing dataset were to be expanded based on digitizing and image classification (without significant changes to the classification scheme), consideration should perhaps be given to the influence of thematic uncertainty on the SHDI metrics and coefficients, as suggested by the higher proportions for $\widehat{\bar{\beta}}_{\text {SHDI }}$ (Table 4.10). The uncertainty in SHDI, the only metric that was influenced by thematic uncertainty (in the image classification), would likely have been higher had crop types in the year-2012 dataset been based on image classification as well. Modelling approaches that consider measurement uncertainties in independent variables are briefly discussed later, in Section 5.4.3.

The change in proportion of $\sigma_{\text {sim }}^{2}$ to $\widehat{\operatorname{var}}\left(\hat{\bar{\theta}}_{\text {sim }}\right)$ for $\widehat{\bar{\beta}}_{\text {P.AG }}$ and $\widehat{\bar{\beta}}_{\text {MFS }}$ across the simulation approaches (little change, relative to that of $\widehat{\bar{\beta}}_{\text {SHDI }}$; Table 4.10) was not surprising, given that these simulation approaches dealt specifically with spatially correlated thematic error, which should have had no impact on the P.AG and MFS metrics. The changes in the proportions for $\widehat{\bar{\beta}}_{\text {P.AG }}$ and $\widehat{\bar{\beta}}_{\text {MFS }}$ are likely due to the slight interaction of P.AG and MFS with SHDI. The larger changes in the proportion for $\widehat{\bar{\beta}}$ SHDI across simulation approaches (average proportion increased $89 \%$ between independent field and landscape grouped simulation; Table 4.10) highlight the fact that the estimates of the influence of positional and thematic uncertainties can be highly dependent on the assumptions surrounding spatially correlated error.

\subsection{General limitations}

This section briefly discusses some of the limitations of this study that were more general than those discussed in the preceding sections. These include limited imagery 
availability, the scenario adopted involving no reference data within the landscapes, and the difficulty in validating some of the uncertainty representations.

Aside from the influence that imagery limitations had on classification accuracy (Sections 5.1.1 and 5.2.1), they also influenced the types of analyses that could be performed. The study area was a good candidate for OBIA (Pasher et al. 2013), which could have provided some opportunities to look into positional and thematic uncertainties in maps that had been segmented and classified with more autonomy than maps based on manual digitizing and classification. However, the only image set that had near-complete coverage of all LCs, across both years, was the air photos (one landscape did not have coverage). OBIA based on only the air photos would likely have been problematic due to limited spectral information (Geneletti \& Gorte, 2003). On the other hand, OBIA based solely on TM imagery (full coverage for year-2011) would have been problematic due to a combination of spectral similarity between some crops and non-agricultural vegetation, and edge widths that were often much smaller than the pixel size. A possible workaround to this issue is to use a combination of segmentation, based on the air photos, and subsequent classification based on the TM imagery (Geneletti \& Gorte, 2003). However, given the similarities in some agricultural and non-agricultural land covers, some interpretation may still be required to isolate agricultural segments.

The scenario adopted wherein no reference data were available within the landscapes (or LCs) limited the simulation approaches that could be used. Had reference pixels been distributed throughout each landscape, SIS could have been a useful approach to accounting for the spatially correlated thematic error (Section 2.5.2). However, the effectiveness of SIS can be dependent on the number of reference locations (e.g. image 
pixels) used and the distribution of these locations, as the degree of structure preservation that it provides can deteriorate at greater distances from the initial conditioning set (de Bruin, 2000; Magnussen \& de Bruin, 2003). In this study, inclusion of several appropriately-distributed reference pixels from the ground referenced maps would have provided some interesting opportunities for simulation approach comparisons. However, given that the maps consisted of reasonably well-delineated boundaries within which land cover could be assumed to be homogenous, having reference pixels distributed throughout each landscape would roughly equate to a ground referenced map.

There was limited validation as to how well the positional and thematic uncertainties were represented. For vertex position uncertainty, the variance in area of the simulated boundaries was found to roughly correspond with that of the re-digitized boundaries, but the re-digitized boundaries were not completely independent of those vertices used to establish the uncertainty estimates (two to four vertices used from each; Section 3.4.1). Ideally, the validation set would have been completely independent. For the representation of boundary interpretation uncertainty, there was little validation. Ideally, multiple digitizers would have digitized a set of fields containing uncertain boundaries, and the frequency with which the uncertain boundaries were included or excluded would be compared to the probability estimates that were based on the edge detection. However, better validation of these forms of uncertainty would have required more time and resources. Finally, the image classifications were validated in terms of thematic accuracy, but the relationships between entropy in the class membership probabilities and thematic error were not fully characterized and accounted for, due to time limitations. 


\subsection{Further research}

There is a lot of room to expand on the research presented in this thesis, including the improvement of the positional and thematic uncertainty estimates (Section 5.4.1), research into alternate simulation approaches that may better account for spatially correlated positional or thematic error (Section 5.4.2), and research into alternate landscape metrics and modelling approaches (Section 5.4.3).

\subsubsection{Improvement of positional and thematic uncertainty estimates}

There is room for improvement in the representation of all forms of positional and thematic uncertainties considered in this study.

Characterizing the degree of correlated error between vertices within a field and considering it during simulation (Shi \& Liu, 2000; Tong et al. 2013), could provide a better representation of the variance in field area associated with vertex positioning uncertainty (Section 5.2.2.1). Additionally, as discussed earlier, using multiple interpreters to produce multiple digitized sets (independent sets for measuring vertex position uncertainty and validation) would likely produce more accurate estimates of vertex position uncertainty.

Of the three forms of uncertainty considered, the boundary interpretation uncertainty estimates perhaps have the most room for improvement. Even with split and merge probabilities based on edge detection, they were still subject to interpretations of what constitutes high or low visibility and to differences between air photo characteristics (Section 5.2.2.2). Further research into alternate approaches for identifying uncertain 
boundaries and assigning probability estimates could be beneficial. For example, Aplin \& Atkinson (2004) presented a potentially useful alternative wherein fields that likely had missing boundaries were identified based on a comparison of modal class proportion within each field to measures of local variability from imagery. Also, a move to automated image segmentation for the delineation of fields would open new research opportunities in terms of representing boundary positioning uncertainty within this dataset.

For thematic uncertainty, there are ways in which the classifications could be improved in terms of classification accuracy and reduced class assignment ambiguity (Section 5.2.1). For example, the grass-like classes were a major source of thematic uncertainty, and differentiating between them with TM imagery alone was problematic (Table 4.6). The incorporation of additional imagery, such as synthetic aperture radar (SAR) imagery, can improve crop classification accuracy (McNairn et al. 2009), though AAFC's Annual Crop Inventory, which used SAR and optical imagery from multiple dates, still felt it necessary to combine Pasture and Forage (e.g. Hay and Legumes) classes in order to achieve the desired accuracy (AAFC, n.d.). In this study, merging some or all of the grass-like classes would likely be beneficial in terms of accuracy (Tables E.26 - E.29), and an improved relationship between entropy in the class membership probabilities and thematic error (Foody, 1996; Section 5.2.1.2), though it would involve changing the nature of the dataset (altered SHDI metrics).

Foody (1996) suggested that accuracy measures based on the comparison of classification output with ground data (e.g. accuracy measures associated with the error matrix) are a better measure of classification quality than entropy, when there is a 
mixture of land covers within pixels (e.g. even correctly classified instances of the grasslike classes had higher entropy due to a mix of similar vegetation types within pixels). As mentioned in Section 5.2.1.2, using global uncertainty estimates would likely have been problematic, given that the degree of uncertainty may vary throughout the study area. However, there are alternatives to relying solely on global accuracy measures. For example, Foody (2005) generated error measures based on local subsets of the validation data and interpolated them, mapping the distribution of these measures throughout the study area. Alternatively, Cockx, Van de Voorde, \& Canters (2014) used global class probabilities from the error matrix, weighted on object-level posterior probabilities of class membership obtained from the classifier. Given the separability issues encountered in this study, thematic uncertainty estimates based on approaches like those just mentioned, could have been more related to thematic error.

\subsubsection{Alternate simulation approaches}

The consideration of spatially correlated thematic error when generating landscape realizations was a big issue throughout this study. The grouped simulation approaches used were rough attempts to consider spatial autocorrelation, particularly in the between-field case. Had the scenario involving no reference data within the landscapes not been adopted, the use of SIS would have provided an interesting opportunity for comparison (Sections 2.5.2 and 5.3). Performing SIS based on an initial

conditioning set of pixels that exhibit low $E_{\mathrm{n}}$ may be possible (e.g. a separate random selection of low $E_{\mathrm{n}}$ pixels used as an initial conditioning set for each realization). There 
may be additional issues with the preservation of the shape of the agricultural fields under SIS, which is often a pixel-based approach (Strebelle, 2002; Gebbers \& de Bruin, 2010). However, aggregating the simulated pixels to the field level, based on majority, for each realization could help to remedy this. Alternatively, more complex object-based or multipoint simulation methods could be useful (Strebelle, 2002; Gebbers \& de Bruin, 2010).

\subsubsection{Alternate metrics and modelling approaches}

Duro et al. (2014) compared the three discrete metrics used in the Farmland Biodiversity Project models (P.AG, MFS, and SHDI) with several continuous metrics derived from the TM imagery, in terms of their ability to explain the variance in the biodiversity metrics. The study found that continuous variables based on NDVI, local variability in NDVI, and local measures of spatial autocorrelation in NDVI tended to perform better than the discrete metrics. Analyzing the uncertainty in continuous variables derived from RS imagery could potentially be performed with sequential Gaussian simulation (similar to SIS but for continuous variables with normally distributed PDFs), but would likely require reference NDVI samples distributed throughout the landscapes (Gebbers \& de Bruin, 2010).

Finally, regardless of whether discrete or continuous metrics are being used, if they are derived from imagery and contain considerable measurement error, then the model coefficients may be biased towards zero (attenuated) (Stefanski, 2000). In such cases, errors-in-variables regression techniques (also called total least squares regression, or orthogonal regression) have been shown to reduce the bias in model coefficients and 
confidence intervals (Markovsky \& Van Huffel, 2007; Lamadrid-Figueroa, Téllez-Rojo, Ángeles, Hernández-Ávila, \& Hu, 2011). Since simulated estimates of measurement uncertainty in the image classification-based SHDI metrics were generated in this study, and since ground referenced SHDI metrics are available, there may be an opportunity to compare the results of ordinary least squares regression and errors-in-variables regression with the image-based metrics, in terms of how they match the results of ordinary least squares regression with the ground referenced metrics. 


\section{Conclusions}

Similar to the findings of Canters (1997), field-based simulation provided better preservation of within-field structure and resulted in greater variance in class proportions across realizations, relative to independent pixel-based simulation. The homogenous field-based simulation approach had, not surprisingly, perfect within-field structure preservation and greater variance in class proportions across realizations than the independent pixel-based and field-based approaches. All three of these simulation approaches exhibited bias in terms of a tendency for the SHDI distributions to be offset towards greater diversity, from both the hard metrics (based on hard image classification) and the reference metrics (based on the ground referenced maps). The offsets from the hard metrics were likely a result of failure to account for spatially correlated thematic error, both within fields (under the independent pixel-based approach) and between fields (under all three approaches). The offsets from the reference metrics were likely influenced by both the spatial autocorrelation issue and classification accuracy.

For area-based diversity metrics, like SHDI, failure to account for spatially correlated thematic error can result in an underrepresentation of the variance that is associated with thematic uncertainty. This underrepresentation can be more of an issue when working with spectrally similar classes, where there is more ambiguity in the class membership probabilities and greater structure breakdown in realizations. Other studies, involving similar landscapes, where local reference data are not available but thematically homogenous areas have been reasonably well-defined (crisp spatial transitions in thematic class, as opposed to gradual), should consider either field-based 
simulation approaches or consider treating the fields as homogenous objects in terms of thematic class. These approaches allow for the consideration of within-field spatial autocorrelation. However, they do not consider between-field spatially correlated thematic error which, if significant, can lead to variance underrepresentation and inflated diversity metrics.

Studies in similar landscapes, that find a significant degree of spatial autocorrelation between fields, should consider simulating the thematic class of nearby, similar fields with common PRNs. At the landscape level, this approach reduces the effects of landscape structure breakdown associated with a failure to consider spatially correlated thematic error between fields. However, the use of common PRNs across fields is more difficult to justify than their use within assumed-homogenous fields. Further research into approaches that consider spatially correlated thematic error, without reliance on local reference data, could be beneficial to future studies in similar landscapes.

Simple MC approaches (compared to approaches such as SIS) were used to obtain rough estimates of the effects of positional and thematic uncertainties in digitized maps on landscape metrics, as well as their effects on biodiversity model coefficients that were based on these metrics. Positional uncertainties were considered separately from thematic uncertainties when generating map realizations. They were broken down into global estimates of uncertainty in vertex positioning, and local uncertainties associated with interpreting the boundaries of individual fields. Thematic uncertainties were based on probability estimates provided by the classifier, with between-field spatial 
autocorrelation considered by grouping similar fields, and generating class realizations using common PRNs within groups.

The influence of the positional and thematic (only for year 2011) uncertainties in the maps on model coefficients was low, relative to the influence of the sample and model selection. The uncertainty estimates of this study put these relative influences at up to $2.5 \%$ for $\widehat{\bar{\beta}}_{\text {P.AG }}$, up to $3.9 \%$ for $\widehat{\bar{\beta}}_{\mathrm{MFS}}$, and up to $28.0 \%$ for $\widehat{\bar{\beta}}_{\mathrm{SHDI}}$, on average, across all 23 models. In terms of the significance of the model coefficients (at 95\% confidence), considering these uncertainties only made a difference for $\widehat{\bar{\beta}}_{\text {MFS }}$ of the beetles abundance model (no longer significant). At the $95 \%$ confidence level, $\widehat{\widehat{\beta}}_{\text {SHDI }}$ was not significant for any of the models, so the consideration of thematic uncertainties made no difference in terms of significance.

If the dataset of the Farmland Biodiversity Project were to be expanded, spatially or temporally, based on image digitizing and classification, the influence of the positional and thematic uncertainties would likely be less than that of the sample and model selection. If digitizing were based on high resolution air photos, the relative influence positional uncertainties on model coefficients would likely be low. Without classification scheme revision, the influence of thematic uncertainty would likely be more comparable to the influences of the sample and model selection uncertainty, but would only influence $\widehat{\bar{\beta}}_{\text {SHDI }}$ (assuming no other major changes in map production methods). Additional imagery and imagery-derived variables (e.g. texture measures) could improve classification accuracy and reduce class assignment ambiguity, though likely not as much as classification scheme revisions, such as the merging of spectrally similar classes. 


\section{References}

AAFC (n.d.). ISO 19131 AAFC Annual Crop Inventory - Data Product Specifications (Revision A) [online document]. Ottawa, CAN: Agriculture and Agri-Food Canada. Available at: http://www.agr.gc.ca/atlas/supportdocument_documentdesupport/aafcCropTypeMapping/en/I

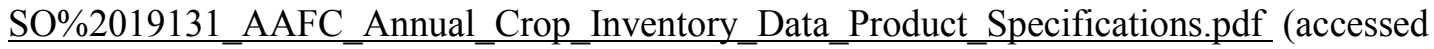
August 21, 2014)

AAFC (2013). AAFC Annual Crop Inventory [web page]. Ottawa, CAN: Agriculture and AgriFood Canada. Available at: http://open.canada.ca/data/en/dataset/ba2645d5-4458-414d-b196-6303ac06c1c9 (accessed November 24, 2014)

Anderson, D.R. (2008). Model Based Inference in the Life Sciences: A Primer on Evidence. New York, US: Springer. doi: 10.1007/978-0-387-74075-1

Aplin, P., \& Atkinson, P. M. (2004). Predicting missing field boundaries to increase per-field classification accuracy. Photogrammetric Engineering \& Remote Sensing, 70(1), 141-149. doi: 10.14358/PERS.70.1.141

Arsham, H., \& Lovric, M. (2011). Bartlett's Test. In M. Lovric (Ed.), International encyclopedia of statistical science (pp. 87-88). Berlin, DE; Heidelberg, DE: Springer-Verlag.

Atkinson, P.M., \& Foody, G.M. (2002). Uncertainty in Remote Sensing and GIS: Fundamentals. In Foody, G. M. \& Atkinson, P. M. (Eds.), Uncertainty in remote sensing and GIS (pp. 1-18). Chichester, UK: John Wiley \& Sons.

Awange, J.L., \& Kyalo Kiema, J.B.K. (2013). Environmental Geoinformatics: Monitoring and Management. Environmental Science and Engineering. Berlin, DE; Heidelberg, DE: Springer. doi: 10.1007/978-3-642-34085-7

Blaes, X., Vanhalle, L., \& Defourny, P. (2005). Efficiency of crop identification based on optical and SAR image time series. Remote Sensing of Environment, 96(3), 352-365.

doi: $10.1016 /$ j.rse.2005.03.010

Blaschke, T. (2010). Object based image analysis for remote sensing. ISPRS journal of photogrammetry and remote sensing, 65(1), 2-16. doi: 10.1016/j.isprsjprs.2009.06.004

Blaschke, T., Hay, G.J., Kelly, M., Lang, S., Hofmann, P., Addink, E., Feitosa, R.Q., van der Meer, F., van der Werff, H., van Coillie, F. \& Tiede, D. (2014). Geographic Object-Based Image Analysis - Towards a new paradigm. ISPRS Journal of Photogrammetry and Remote Sensing, 87, 180-191. doi: 10.1016/j.isprsjprs.2013.09.014

Bhatta, B. (2011). Remote sensing and GIS (2nd ed.). New Delhi, IN: Oxford University Press. 
Bogaert, J., Rousseau, R., Van Hecke, P., \& Impens, I. (2000). Alternative area-perimeter ratios for measurement of 2D shape compactness of habitats. Applied Mathematics and Computation, 111(1), 71-85. doi: 10.1016/S0096-3003(99)00075-2

Breiman, L. (2001). Random forests. Machine Learning, 45(1), 5-32. doi: 10.1023/A:1010933404324

Breiman, L., Friedman, J., Olshen, R.A., \& Stone, C.J. (1984). Classification and regression trees. Belmont, US: Wadsworth, Inc.

Breiman, L., Cutler, A., Liaw, A., \& Wiener, M. (2012). Breiman and Cutler's random forests for classification and regression. R package version 4.6-7. Available at: http://cran.r-project.org/web/packages/randomForest/randomForest.pdf (accessed July 16, 2013)

Brown, J. D. \& Heuvelink, G. B. M. (2007). The Data Uncertainty Engine (DUE): A software tool for assessing and simulating uncertain environmental variables. Computers \& Geosciences, 33(2), 172-190. doi: 10.1016/j.cageo.2006.06.015

Brown, K. M., Foody, G. M., \& Atkinson, P. M. (2009). Estimating per-pixel thematic uncertainty in remote sensing classifications. International Journal of Remote Sensing, 30(1), 209-229. doi: 10.1080/01431160802290568

Burnham, K.P. \& Anderson, D.R. (2002). Model Selection and Multimodel Inference: A Practical Information-Theoretic Approach (2nd ed.). New York, US: Springer.

Caers, J. (2011). Modeling uncertainty in the earth sciences. Chichester, UK: John Wiley \& Sons.

Castillejo-González, I.L., López-Granados, F., García-Ferrer, A., Peña-Barragán, J.M., JuradoExpósito, M., de la Orden, M.S., \& González-Audicana, M. (2009). Object- and pixel-based analysis for mapping crops and their agro-environmental associated measures using QuickBird imagery. Computers and Electronics in Agriculture, 68(2), 207-215.

doi: 10.1016/j.compag.2009.06.004

Canters, F. (1997). Evaluating the Uncertainty of Area Estimates Derived from Fuzzy LandCover Classification. Photogrammetric Engineering \& Remote Sensing, 63(4), 403-414.

Canters, F., De Genst, W., \& Dufourmont, H. (2002). Assessing effects of input uncertainty in structural landscape classification. International Journal of Geographical Information Science, 16(2), 129-149. doi: 10.1080/13658810110099143

Centers for Disease Control and Prevention (2008). Epi Info 7: Administrative shapefiles [Vector Digital Data]. Layers: North America. Atlanta, US: Centers for Disease Control and Prevention. Available at:

http://wwwn.cdc.gov/epiinfo/html/shapefiles.htm (accessed April 2, 2014)

Cockx, K., Van de Voorde, T., \& Canters, F. (2014). Quantifying uncertainty in remote sensingbased urban land-use mapping. International Journal of Applied Earth Observation and Geoinformation, 31, 154-166. doi: 10.1016/j.jag.2014.03.016 
Congalton, R.G., \& Green, K. (2009). Assessing the Accuracy of Remotely Sensed Data: Principles and Practices (2nd ed.). Boca Raton, US: CRC Press.

Corvalan, C., Hales, S., McMichael, A., Butler, C., Campbell-Lendrum, D., Confalonieri, U., Leitner, K., Lewis, N., Patz, J., Polson, K., Scheraga, J., Woodward, A., Younes, M. et al. (2005). Ecosystems and human well-being: Health synthesis - A report of the Millennium Ecosystem Assessment [Online document]. Geneva, Switzerland: World Health Organization. Available at:

http://www.who.int/entity/globalchange/ecosystems/ecosys.pdf?ua=1 (accessed August 21, 2014)

Crist, T.O., Veech, J.A., Gering, J.C., \& Summerville, K.S. (2003). Partitioning species diversity across landscapes and regions: a hierarchical analysis of $\alpha, \beta$, and $\gamma$ diversity. The American Naturalist, 162(6), 734-743. doi: 10.1086/378901

Crosetto, M., Tarantola, S., \& Saltelli, A. (2000). Sensitivity and uncertainty analysis in spatial modelling based on GIS. Agriculture, Ecosystems \& Environment, 81(1), 71-79. doi: 10.1016/S0167-8809(00)00169-9

Crosetto, M., \& Tarantola, S. (2001). Uncertainty and sensitivity analysis: tools for GIS-based model implementation. International Journal of Geographical Information Science, 15(5), 415-437. doi: 10.1080/13658810110053125

Crosetto, M., Ruiz, J. A. M., \& Crippa, B. (2001). Uncertainty propagation in models driven by remotely sensed data. Remote Sensing of Environment, 76(3), 373-385. doi: 10.1016/S0034-4257(01)00184-5

Cutler, A., Cutler, D., \& Stevens, J.R. (2012). Random Forests. In C. Zhang \& Y. Ma (Eds.), Ensemble Machine Learning: Methods and Applications (pp. 157-175). New York, US: Springer. doi: 10.1007/978-1-4419-9326-7

Davidson, A., \& Csillag, F. (2001). The influence of vegetation index and spatial resolution on a two-date remote sensing-derived relation to $\mathrm{C} 4$ species coverage. Remote Sensing of Environment, 75 (1), 138-151. doi: 10.1016/S0034-4257(00)00162-0

de Bruin, S. (2000). Predicting the Areal Extent of Land-Cover Types Using Classified Imagery and Geostatistics. Remote Sensing of Environment, 74(2), 387-396.

doi: 10.1016/S0034-4257(00)00132-2

de Bruin, S., Heuvelink, G.B.M., \& Brown, J.D. (2008). Propagation of positional measurement errors to agricultural field boundaries and associated costs. Computers and Electronics in Agriculture, 63(2), 245-256. doi: 10.1016/j.compag.2008.03.005

De Wit, A.J.W., \& Clevers, J.G.P.W. (2004). Efficiency and accuracy of per-field classification for operational crop mapping. International Journal of Remote Sensing, 25(20), 4091-4112. doi: 10.1080/01431160310001619580 
Dingle Robertson, L., \& King, D.J. (2011). Comparison of pixel- and object-based classification in land cover change mapping. International Journal of Remote Sensing, 32(6), 1505-1529. doi: $10.1080 / 01431160903571791$

Digital Globe (n.d.). QuickBird Data Sheet [online document]. Longmont, US: Digital Globe. Available at: https://www.digitalglobe.com/sites/default/files/QuickBird-DS-QB-Prod.pdf (accessed November 24, 2014)

DMTI Spatial (2012). DMTI National CanMap Suite [Vector Digital Data]. Version 2012.3. Layers: CanMap Highways, Major Water Regions (MJWTR), Major Places (MJPPN). Markham, CAN: DMTI Spatial Inc. Available at: http://www.dmtispatial.com/Software-And-Data/CanMap.html (accessed November 24, 2014)

Dungan, J.L. (2002). Toward a comprehensive view of uncertainty in remote sensing analysis. In Foody, G. M. \& Atkinson, P. M. (Eds.), Uncertainty in Remote Sensing and GIS (pp. 2535). Chichester, UK: John Wiley \& Sons.

Duro, D.C., Franklin, S.E., \& Dubé, M.G. (2012). A comparison of pixel-based and object-based image analysis with selected machine learning algorithms for the classification of agricultural landscapes using SPOT-5 HRG imagery. Remote Sensing of Environment, 118, 259-272. doi: $10.1016 /$ j.rse.2011.11.020

Duro, D.C., Girard, J., King, D.J., Fahrig, L., Mitchell, S., Lindsay, K., Tischendorf, L. (2014). Predicting species diversity in agricultural environments using Landsat TM imagery. Remote Sensing of Environment, 144, 214-225. doi: 10.1016/j.rse.2014.01.001

Eastman, R. (2001). Uncertainty management in GIS: Decision support tools for effective use of spatial data resources. In C. T. Hunsaker, M. F. Goodchild, M. A. Friedl, \& T. J. Case (Eds.), Spatial uncertainty in ecology: Implications for remote sensing and GIS applications (pp. 379390). New York, US: Springer.

Eastman, J.R. (2009a). IDRISI Taiga. Worcester, US: Clark University.

Eastman, J.R. (2009b). IDRISI Taiga Help System: BAYCLASS. Worcester, US: Clark University.

Eastman, J.R. (2012). IDRISI Selva Help System: BAYCLASS. Worcester, US: Clark University. Available at: http://www.clarklabs.org/support/IDRISI-Taiga-Help-System.cfm (accessed November 12, 2014)

Eastman, J.R., \& Laney, R.M. (2002). Bayesian Soft Classification for Sub-Pixel Analysis: A Critical Evaluation. Photogrammetric Engineering \& Remote Sensing, 68(11), 1149-1154.

Edwards, G., \& Lowell, K. E. (1996). Modeling uncertainty in photointerpreted boundaries. Photogrammetric Engineering and Remote Sensing, 62(4), 377-391. 
ESRI (2012). ArcGIS 10.1 for Desktop. Redlands, US: Environmental Systems Research Institute.

ESRI (2013a). ArcGIS 10.2 for Desktop. Redlands, US: Environmental Systems Research Institute.

ESRI (2013b). ArcGIS Help 10.1: Repair Geometry (Data Management) [web page]. Redlands, US: Environmental Systems Research Institute. Available at: http://resources.arcgis.com/en/help/main/10.1/index.html\#//00170000003v000000 (accessed November 26, 2014)

Fahrig, L., Baudry, J., Brotons, L., Burel, F.G., Crist, T.O., Fuller, R. J., Sirami, C., Siriwardena, G.M., \& Martin, J.L. (2011). Functional landscape heterogeneity and animal biodiversity in agricultural landscapes. Ecology Letters, 14(2), 101-112.

doi: $10.1111 /$ j.1461-0248.2010.01559.x

Fahrig, L., Girard, J., Duro, D., Pasher, J., Smith, A., Javorek, S., King, D., Lindsay, K.F., Mitchell, S., \& Tischendorf, L. (2015). Farmlands with smaller crop fields have higher biodiversity. Agriculture, Ecosystems \& Environment, 200, 219-234.

doi: 10.1016/j.agee.2014.11.018

Foody, G.M. (1996). Approaches for the production and evaluation of fuzzy land cover classifications from remotely-sensed data. International Journal of Remote Sensing, 17(7), 1317-1340. doi: 10.1080/01431169608948706

Foody, G.M. (2002). Status of land cover classification accuracy assessment. Remote Sensing of Environment, 80(1), 185-201. doi: 10.1016/S0034-4257(01)00295-4

Foody, G.M. (2005). Local characterization of thematic classification accuracy through spatially constrained confusion matrices. International Journal of Remote Sensing, 26(6), 1217-1228. doi: $10.1080 / 01431160512331326521$

Foody, G.M., Campbell, N.A., Trodd, N.M., \& Wood, T.F. (1992). Derivation and Applications of Probabilistic Measures of Class Membership from the Maximum-Likelihood Classification. Photogrammetric Engineering \& Remote Sensing, 58(9), 1335-1341.

Foody, G.M., Mathur, A., Sanchez-Hernandez, C., \& Boyd, D.S. (2006). Training set size requirements for the classification of a specific class. Remote Sensing of Environment, 104(1), 1-14. doi: $10.1016 /$ j.rse.2006.03.004

Forman, R.T. \& Godron M. (1986). Landscape Ecology. New York, US: Wiley.

Fortin, M.J., \& Edwards, G. (2001). Delineation and Analysis of Vegetation Boundaries. In C. T. Hunsaker, M. F. Goodchild, M. A. Friedl, \& T. J. Case (Eds.), Spatial uncertainty in ecology: Implications for remote sensing and GIS applications (pp. 158-174). New York, US: Springer.

Franklin, J.F., \& Lindenmayer, D.B. (2009). Importance of matrix habitats in maintaining biological diversity. Proceedings of the National Academy of Sciences, 106(2), 349-350. doi: 10.1073/pnas.0812016105 
Friedl, M.A., McGwire, K.C., \& McIver, D.K. (2001). An overview of uncertainty in optical remotly sensed data for ecological applications. In C. T. Hunsaker, M. F. Goodchild, M. A. Friedl, \& T. J. Case (Eds.), Spatial uncertainty in ecology: Implications for remote sensing and GIS applications (pp. 258-283). New York, US: Springer.

Gabriel, D., Sait, S.M., Kunin, W.E., \& Benton, T.G. (2013). Food production vs. biodiversity: comparing organic and conventional agriculture. Journal of Applied Ecology, 50(2), 355-364. doi: $10.1111 / 1365-2664.12035$

Gebbers, R. \& de Bruin, S. (2010). Application of geostatistical simulation in precision agriculture. In Oliver, M. A. (Ed.), Geostatistical applications for precision agriculture (pp. 269-303). Dordrecht, NL; Heidelberg, DE; London, UK; New York, US: Springer. doi: 10.1007/978-90-481-9133-8

Geneletti, D., \& Gorte, B.G.H. (2003). A method for object-oriented land cover classification combining Landsat TM data and aerial photographs. International Journal of Remote Sensing, 24(6), 1273-1286. doi: 10.1080/01431160210144499

Gilks, W.R., Richardson, S., \& Spiegelhalter, D.J. (1996). Introducing Markov chain Monte Carlo. In W. R. Gilks, S. Richardson, \& D. J. Spiegelhalter (Eds.), Markov chain Monte Carlo in Practice (pp. 1-19). London, UK: Chapman \& Hall.

Goodchild, M.F., \& Hunter, G.J. (1997). A simple positional accuracy measure for linear features. International Journal of Geographical Information Science, 11(3), 299-306. doi: $10.1080 / 136588197242419$

Gonçalves, L.M.S., Fonte, C.C., Júlio, E.N.B.S., \& Caetano, M. (2012). The application of uncertainty measures in the training and evaluation of supervised classifiers. International Journal of Remote Sensing, 33(9), 2851-2867. doi: 10.1080/01431161.2011.622315

Green, D.G., Klomp, N., Rimmington, G., \& Sadedin, S. (2006). Complexity in landscape ecology. Dordrecht, NL: Springer.

Haines-Young, R.H., Green, D.R., \& Cousins S. (1993). Landscape ecology and spatial information systems. In Haines-Young, R. H., Green, D. R., \& Cousins S. (Eds.) Landscape ecology and geographic information systems (pp. 3-8). London, UK: Taylor \& Francis.

Hurlbert, S. H. (1971). The nonconcept of species diversity: a critique and alternative parameters. Ecology, 52(4), 577-586.

Jensen, J. R. (1996). Introductory digital image processing: a remote sensing perspective (2nd ed.). London, UK: Prentice-Hall.

Jensen, J.R. (2000). Remote sensing of the environment: An earth resource perspective. Upper Saddle River, US: Prentice-Hall.

Kavzoglu, T., \& Mather, P. M. (2002). The role of feature selection in artificial neural network applications. International Journal of Remote Sensing, 23(15), 2919-2937. doi: 10.1080/01431160110107743 
Kroese, D.P., Taimre, T., \& Botev, Z.I. (2011). Handbook of Monte Carlo Methods. Hoboken,US: John Wiley \& Sons.

Kyriakidis, P. C. \& Dungan, J. L. (2001). A geostatistical approach for mapping thematic classification accuracy and evaluating the impact of inaccurate spatial data on ecological model predictions. Environmental and Ecological Statistics, 8(4), 311-330. doi: 10.1023/A:1012778302005

Lamadrid-Figueroa, H., Téllez-Rojo, M.M., Ángeles, G., Hernández-Ávila, M., \& Hu, H. (2011). Bias correction by use of errors-in-variables regression models in studies with KX-ray fluorescence bone lead measurements. Environmental Research, 111(1), 17-20. doi: $10.1016 /$ j.envres.2010.10.011

Lande, R. (1996). Statistics and partitioning of species diversity, and similarity among multiple communities. Oikos, 76(1), 5-13. doi: 10.2307/3545743

Liang, S., Fang, H., \& Chen, M. (2002). Atmospheric correction of Landsat ETM+ land surface imagery - Part I: Methods. IEEE Transactions on Geoscience and Remote Sensing, 39(11), 2490-2498. doi: 10.1109/36.964986

Lillesand, T. M., Kiefer, R. W.m \& Chipman, J. W. (2004). Remote sensing and image interpretation (5th ed.). Hoboken, US: John Wiley \& Sons.

Loosvelt, L., Peters, J., Skriver, H., Lievens, H., Van Coillie, F.M.B., De Baets, B., \& Verhoest, N.E.C. (2012). Random Forests as a tool for estimating uncertainty at pixel-level in SAR image classification. International Journal of Applied Earth Observation and Geoinformation, 19, 173-184. doi: 10.1016/j.jag.2012.05.011

Magnussen, S., \& de Bruin, S. (2003). Updating cover type maps using sequential indicator simulation. Remote Sensing of Environment, 87(2), 161-170.

doi: 10.1016/S0034-4257(03)00138-X

Markovsky, I., \& Van Huffel, S. (2007). Overview of total least-squares methods. Signal Processing, 87(10), 2283-2302. doi: 10.1016/j.sigpro.2007.04.004

Matsumoto, M., \& Nishimura, T. (1998). Mersenne twister: a 623-dimensionally equidistributed uniform pseudo-random number generator. ACM Transactions on Modeling and Computer Simulation (TOMACS), 8(1), 3-30. doi: 10.1145/272991.272995

McNairn, H., Champagne, C., Shang, J., Holmstrom, D., \& Reichert, G. (2009). Integration of optical and Synthetic Aperture Radar (SAR) imagery for delivering operational annual crop inventories. ISPRS Journal of Photogrammetry and Remote Sensing, 64(5), 434-449. doi: 10.1016/j.isprsjprs.2008.07.006

Menze, B.H., Kelm, B.M., Masuch, R., Himmelreich, U., Bachert, P., Petrich, W., \& Hamprecht, F.A. (2009). A comparison of random forest and its Gini importance with standard chemometric methods for the feature selection and classification of spectral data. BMC bioinformatics, 10(1), 213. doi: 10.1186/1471-2105-10-213 
Minister of Supply and Services Canada (1995). Canadian Biodiversity Strategy: Canada's Response to the Convention on Biological Diversity. Environment Canada Biodiversity Convention Office: Hull, QC. Catalog No. En21-134/1995E. Available at: http:/www.biodivcanada.ca/560ED58E-0A7A-43D8-8754-C7DD12761EFA/CBS_e.pdf (accessed November 10, 2014).

Mitchell, S.W., Remmel, T.K., \& Wulder, M.A. (2008). Distance to second cluster as a measure of classification confidence. Remote Sensing of Environment, 112(5), 2615-2626. doi: $10.1016 /$ j.rse.2007.12.006

Morgan, M.G., Henrion, M., \& Small, M. (1990). Uncertainty: A guide to dealing with uncertainty in quantitative risk and policy analysis. Cambridge, UK; New York, US: Cambridge University Press.

Mowrer, T. H. (1997). Propagating uncertainty through spatial estimation processes for oldgrowth subalpine forests using sequential Gaussian simulation in GIS. Ecological Modelling, 98(8), 73-86. doi: 10.1016/S0304-3800(96)01938-2

Myint, S.W., Gober, P., Brazel, A., Grossman-Clarke, S., \& Weng, Q. (2011). Per-pixel vs. object-based classification of urban land cover extraction using high spatial resolution imagery. Remote Sensing of Environment, 115(5), 1145-1161. doi: 10.1016/j.rse.2010.12.017

NASA Landsat Program (2011). Landsat-5 TM scenes LT50150292011154EDC00, LT50150292011170EDC00, LT50150292011186EDC00, LT50150292011282EDC00. L1T, Standard Terrain Corrected. Sioux Falls, US: United States Geological Survey (USGS). 3 June, 19 June, 5 July, 9 October, 2011.

Natural Resources Canada (2014). Canadian Geopolitical Boundaries (Edition 2.4) [Vector Digital Data]. Layers: Provinces. Ottawa, CAN: Government of Canada, Natural Resources Canada, Surveyor General Branch. Available at: http:/geobase.ca/geobase/en/data/admin/index.html (accessed April 2, 2014)

OMFRA (n.d.) Eastern Ontario region at a glance [online report]. Ontario Ministry of Agriculture, Food, and Rural Affairs. Available at: http://www.omafra.gov.on.ca/english/stats/county/eastern_ontario.pdf (accessed August 21, 2014)

OMFRA (2011a). Ontario Field Crop Report: 2011 Corn Seasonal Summary [online report]. Ontario Ministry of Agriculture, Food, and Rural Affairs. Available at: http://www.omafra.gov.on.ca/english/crops/field/reports/2011summary-corn.htm (accessed June 16, 2014)

OMFRA (2011b). Ontario Field Crop Report: 2011 Soybean Seasonal Summary [online report]. Ontario Ministry of Agriculture, Food, and Rural Affairs. Available at: http://www.omafra.gov.on.ca/english/crops/field/reports/2011 summary-soybean.htm (accessed June 16, 2014) 
OMFRA (2011c). Ontario Field Crop Report: 2011 Forage Seasonal Summary [online report]. Ontario Ministry of Agriculture, Food, and Rural Affairs. Available at: http://www.omafra.gov.on.ca/english/crops/field/reports/2011summary-forage.htm (accessed June 16, 2014)

OMFRA (2011d). Ontario Field Crop Report: 2011 Pasture Seasonal Summary [online report]. Ontario Ministry of Agriculture, Food, and Rural Affairs. Available at: http://www.omafra.gov.on.ca/english/crops/field/reports/2011summary-pasture.htm (accessed June 16, 2014)

OMFRA (2011e). Ontario Field Crop Report: 2011 Cereals Seasonal Summary [online report]. Ontario Ministry of Agriculture, Food, and Rural Affairs. Available at: http://www.omafra.gov.on.ca/english/crops/field/reports/2011 summary-cereals.htm (accessed June 16, 2014)

OMFRA (2012a). Ontario Field Crop Report: 2012 Corn Seasonal Summary [online report]. Ontario Ministry of Agriculture, Food, and Rural Affairs. Available at: http://www.omafra.gov.on.ca/english/crops/field/reports/2012summary-corn.htm (accessed June 16, 2014)

OMFRA (2012b). Ontario Field Crop Report: 2012 Soybean Seasonal Summary [online report]. Ontario Ministry of Agriculture, Food, and Rural Affairs. Available at: http://www.omafra.gov.on.ca/english/crops/field/reports/2012summary-soybean.htm (accessed June 16, 2014)

OMFRA (2012c). Ontario Field Crop Report: 2012 Forages Seasonal Summary [online report]. Ontario Ministry of Agriculture, Food, and Rural Affairs. Available at: http://www.omafra.gov.on.ca/english/crops/field/reports/2012summary-forages.htm (accessed June 16, 2014)

OMFRA (2012d). Ontario Field Crop Report: 2012 Pasture Seasonal Summary [online report]. Ontario Ministry of Agriculture, Food, and Rural Affairs. Available at: http://www.omafra.gov.on.ca/english/crops/field/reports/2012summary-pasture.htm (accessed June 16, 2014)

OMFRA (2012e). Ontario Field Crop Report: 2012 Cereals Seasonal Summary [online report]. Ontario Ministry of Agriculture, Food, and Rural Affairs. Available at: http://www.omafra.gov.on.ca/english/crops/field/reports/2012summary-cereals.htm (accessed June 16, 2014)

Ontario Biodiversity Council (2005). Protecting what sustains us: Ontario's Biodiversity Strategy [online report]. Peterborough, ON: Ontario Biodiversity Council. Available at: http://ontariobiodiversitycouncil.ca/files/2012/03/OBS_2005.pdf (accessed November 10, 2014) 
Ontario Biodiversity Council (2010). State of Ontario's biodiversity 2010: A report of the Ontario Biodiversity Council [online report]. Peterborough, ON: Ontario Biodiversity Council. Available at: http://viewer.zmags.com/publication/6aa599ac (accessed 21 August, 2014)

Ontario Biodiversity Council (2011). Ontario's biodiversity strategy, 2011: Renewing our commitment to protecting what sustains us [online report]. Peterborough, ON: Ontario Biodiversity Council. Available at: http://viewer.zmags.com/publication/c527c66f (accessed August 21, 2014)

Ontario Ministry of Natural Resources (2009). Digital Raster Acquisition Project Eastern Ontario (DRAPE). Peterborough, CAN: Ontario Ministry of Natural Resources. Metadata available via: https://www.javacoeapp.lrc.gov.on.ca/geonetwork/srv/en/main.home (accessed August 20, 2014)

Pal, M. (2005). Random forest classifier for remote sensing classification. International Journal of Remote Sensing, 26(1), 217-222. doi: 10.1080/01431160412331269698

Pasher, J., Mitchell, S.W., King, D.J., Fahrig, L., Smith, A.C., \& Lindsay, K.E. (2013). Optimizing landscape selection for estimating relative effects of landscape variables on ecological responses. Landscape Ecology, 28(3), 371-383. doi: 10.1007/s10980-013-9852-6

Phalan, B., Balmford, A., Green, R.E., \& Scharlemann, J.P.W. (2011). Minimising the harm to biodiversity of producing more food globally. Food Policy, 36(1): S62-S71. doi: 10.1016/j.foodpol.2010.11.008

Prevedello, J.A., \& Vieira, M.V. (2010). Does the type of matrix matter? A quantitative review of the evidence. Biodiversity and Conservation, 19(5), 1205-1223. doi: $10.1007 / \mathrm{s} 10531-009-9750-\mathrm{z}$

Qi, Y. (2012). Random Forest for Bioinformatics. In C. Zhang \& Y. Ma (Eds.), Ensemble Machine Learning: Methods and Applications (pp. 307-323). New York, US: Springer. doi: 10.1007/978-1-4419-9326-7

Questier, F., Put, R., Coomans, D., Walczak, B., \& Heyden, Y.V. (2005). The use of CART and multivariate regression trees for supervised and unsupervised feature selection. Chemometrics and Intelligent Laboratory Systems, 76(1), 45-54. doi: 10.1016/j.chemolab.2004.09.003

Remmel, T.K., Ko, C., \& Bello, R.L. (2010). Characterizing and comparing GIS polygon shape complexity by iterative shinking spectra. In R. Devillers, \& H. Goodchild (Eds.), Spatial data quality: from process to decisions (pp. 41-52). Boca Raton, US: CRC Press.

Richter, R. (1996). A spatially adaptive fast atmospheric correction algorithm. International Journal of Remote Sensing, 17(6), 1201-1214. doi: 10.1080/01431169608949077

Shao, G., \& Wu, J. (2008). On the accuracy of landscape pattern analysis using remote sensing data. Landscape Ecology, 23(5), 505-511. doi: 10.1007/s10980-008-9215-x 
Shi, W. (1998). A generic statistical approach for modelling error of geometric features in GIS. International Journal of Geographical Information Science, 12(2), 131-143. doi: $10.1080 / 136588198241923$

Shi, W., Ehlers, M., \& Tempfli, K. (1999). Analytical Modelling of Positional and Thematic Uncertainties in the Integration of Remote Sensing and Geographical Information Systems. Transactions in GIS, 3(2): 119-136. doi: 10.1111/1467-9671.00011

Shi, W., \& Liu, W. (2000). A stochastic process-based model for the positional error of line segments in GIS. International Journal of Geographical Information Science, 14(1), 51-66. doi: $10.1080 / 136588100240958$

Smith, J.H., Wickham, J.D., Stehman, S.V., \& Yang, L. (2002). Impacts of patch size and landcover heterogeneity on thematic image classification accuracy. Photogrammetric Engineering and Remote Sensing, 68(1), 65-70.

Song, C., Woodcock, C.E., Seto, K.C., Lenney, M.P., \& Macomber, S.A. (2001). Classification and change detection using Landsat TM data: when and how to correct atmospheric effects? Remote sensing of Environment, 75(2), 230-244. doi: 10.1016/S0034-4257(00)00169-3

Statistics Canada (2011). 2011 Census of Agriculture: Farm and Farm Operator Data. Catalogue no. 95-640-XWE. Available at: http://www29.statcan.gc.ca/ceag-web/eng/index-index (accessed August 21, 2014)

Stefanski, L. A. (2000). Measurement error models. Journal of the American Statistical Association, 95(452), 1353-1358. doi: 10.1080/01621459.2000.10474347

Stine, P.A. \& Hunsaker, C.T. (2001). An introduction to uncertainty issues for spatial data used in ecological applications. In C. T. Hunsaker, M. F. Goodchild, M. A. Friedl, \& T. J. Case (Eds.), Spatial uncertainty in ecology: Implications for remote sensing and GIS applications (pp. 91-107). New York, US: Springer.

Strebelle, S. (2002). Conditional simulation of complex geological structures using multiple-point statistics. Mathematical Geology, 34(1), 1-21. doi: 10.1023/A:1014009426274

The R Core Team (2013). R: A Language and Environment for Statistical Computing. [Version 3.0.1 (2013-05-16)]. The R Foundation for Statistical Computing.

The R Core Team (2014). R: A Language and Environment for Statistical Computing Reference Index. [Version 3.1.1 (2014-07-10)]. The R Foundation for Statistical Computing. Available at:

http://cran.r-project.org/doc/manuals/r-release/fullrefman.pdf (accessed August 21, 2014)

Thomlinson, J.R., Bolstad, P.V., \& Cohen, W.B. (1999). Coordinating methodologies for scaling landcover classifications from site-specific to global: Steps toward validating global map products. Remote Sensing of Environment, 70(1), 16-28.

doi: 10.1016/S0034-4257(99)00055-3 
Thompson, K.M. \& Graham, J. D. (1996). Going beyond the single number: Using probabilistic risk assessment to improve risk management. Human and Ecological Risk Assessment, 2(4), 1008-1034. doi: 10.1080/10807039609383660

Tong, X., Sun, T., Fan, J., Goodchild, M.F., \& Shi, W. (2013). A statistical simulation model for positional error of line features in Geographic Information Systems (GIS). International Journal of Applied Earth Observation and Geoinformation, 21, 136-148. doi: $10.1016 /$ j.jag.2012.08.004

Tso, B., \& Mather, P.M. (2009). Classification methods for remotely sensed data (2nd ed.). Boca Raton, US: Taylor \& Francis.

Tuominen, S., \& Pekkarinen, A. (2004). Local radiometric correction of digital aerial photographs for multi source forest inventory. Remote Sensing of Environment, 89(1), 72-82. doi: $10.1016 /$ j.rse.2003.10.005

United Nations (1992). Convention on Biological Diversity. Available at: https://www.cbd.int/doc/legal/cbd-en.pdf (accessed November 10, 2014)

USGS (n.d. a). Landsat Missions: Landsat Processing Details [web page]. Reston, US: United States Geological Survey (USGS). Available at: http://landsat.usgs.gov/Landsat_Processing_Details.php (accessed November 24, 2014)

USGS (n.d. b). Landsat Missions: Landsat 5 History [web page]. Reston, US: United States Geological Survey (USGS). Available at: http://landsat.usgs.gov/about_landsat5.php (accessed November 24, 2014)

USGS (n.d. c). Landsat Missions: SLC-off Products -- Background [web page]. Reston, US: United States Geological Survey (USGS). Available at: http://landsat.usgs.gov/products_slcoffbackground.php (accessed November 24, 2014)

Uuemaa, E., Mander, Ü., \& Marja, R. (2013). Trends in the use of landscape spatial metrics as landscape indicators: a review. Ecological Indicators, 28, 100-106.

doi: 10.1016/j.ecolind.2012.07.018

van den Berg (2012). Fuzzy classification for Eastern Ontario land cover mapping (Master's thesis, Carleton University, Ottawa, Ontario). Retrieved from ProQuest Dissertations \& Theses: http://www.proquest.com/products-services/dissertations/ (accessed November 22, 2014)

Van Niel, T.G., McVicar, T.R., \& Datt, B. (2005). On the relationship between training sample size and data dimensionality: Monte Carlo analysis of broadband multi-temporal classification. Remote Sensing of Environment, 98(4), 468-480.

doi: $10.1016 /$ j.rse.2005.08.011

Vasseur, C., Joannon, A., Aviron, S., Burel, F., Meyneard, J.M. \& Baudry, J. (2013). The cropping systems mosaic: How does the hidden heterogeneity of agricultural landscapes drive arthropod populations?. Agriculture, Ecosystems, and Environment, 166, 3-14. doi: $10.1016 /$ j.agee.2012.08.013 
Wagner, H.H., Wildi, O., \& Ewald, K.C. (2000). Additive partitioning of plant species diversity in an agricultural mosaic landscape. Landscape Ecology, 15(3), 219-227.

doi: $10.1023 / \mathrm{A}: 1008114117913$

Wang, F. (1990). Fuzzy supervised classification of remote sensing images. IEEE Transactions on Geoscience and Remote Sensing, 28(2), 194-201.

Whittaker, R.J., Willis, K.J., \& Field, R. (2001). Scale and species richness: towards a general, hierarchical theory of species diversity. Journal of Biogeography, 28(4), 453-470. doi: 10.1046/j.1365-2699.2001.00563.x

Wicks, T.E., Smith, G.M., \& Curran, P.J. (2002). Polygon-based aggregation of remotely sensed data for regional ecological analyses. International Journal of Applied Earth Observation and Geoinformation, 4(2), 161-173. doi: 10.1016/S0303-2434(02)00025-9

Zhang, J., \& Goodchild, M.F. (2002). Uncertainty in Geographical Information. London, UK: Taylor \& Francis. 


\section{Appendix A: Example of independent pixel-based versus field-based simulation}

Independent pixel-based simulation Independent PRNs

A

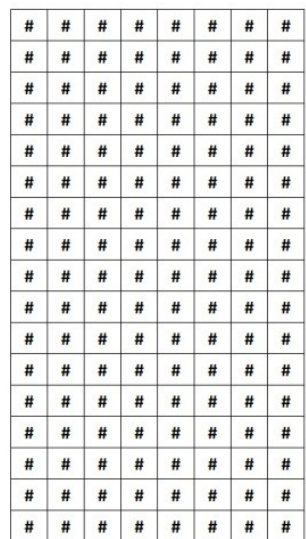

Independent PRNs

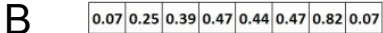
0.000 .480 .470 .080 .120 .680 .820 .56 0.870 .910 .440 .710 .470 .170 .690 .53 0.100 .230 .820 .130 .540 .610 .100 .48 0.890 .990 .700 .590 .650 .140 .710 .93 0.430 .670 .180 .030 .390 .650 .240 .48 0.680 .490 .880 .130 .650 .960 .200 .65 0.880 .150 .790 .130 .430 .440 .380 .56 0.030 .330 .830 .950 .370 .490 .240 .33 $\begin{array}{llllllllllllllll}0.13 & 0.34 & 0.43 & 0.73 & 0.81 & 0.60 & 0.25 & 0.52\end{array}$ \begin{tabular}{llllllllllllll}
\hline 0.99 & 0.22 & 0.12 & 0.20 & 0.30 & 0.67 & 0.81 & 0.56 \\
\hline
\end{tabular} 0.940 .590 .110 .040 .110 .760 .680 .48 0.280 .530 .010 .770 .980 .820 .900 .05 $0.83 \quad 0.00 \quad 0.390 .440 .180 .87 \quad 0.750 .52$ $0.550 .840 .50 \quad 0.210 .040 .290 .150 .78$ 0.830 .140 .160 .290 .050 .100 .650 .24 $\begin{array}{lllllllllllll}0.94 & 0.93 & 0.69 & 0.12 & 0.77 & 0.87 & 0.89 & 0.51\end{array}$

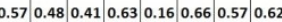
0.390 .550 .790 .800 .130 .980 .180 .39 $0.16 \quad 0.770 .030 .58 \quad 0.060 .720 .620 .47$ $\begin{array}{lllllllllll}0.89 & 0.16 & 0.63 & 0.45 & 0.40 & 0.29 & 0.30 & 0.97\end{array}$ $0.350 .460 .010 .950 .150 .53 \quad 0.220 .53$ 0.140 .420 .980 .450 .020 .470 .190 .02 $0.390 .83 \quad 0.180 .130 .820 .24 \quad 0.80 \quad 0.04$ $0.990 .880 .710 .400 .08 \quad 0.600 .850 .33$

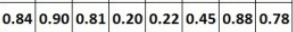
0.880 .160 .160 .620 .330 .080 .960 .28 $\begin{array}{lllllllllllllll}0.77 & 0.62 & 0.99 & 0.75 & 0.20 & 0.28 & 0.78 & 0.69\end{array}$ 0.340 .440 .510 .320 .300 .520 .180 .56 $0.490 .96 \quad 0.97 \quad 0.260 .40 \quad 0.24 \quad 0.740 .52$ $\begin{array}{llllllllll}0.21 & 0.67 & 0.17 & 0.16 & 0.89 & 0.13 & 0.28 & 0.89\end{array}$ 0.410 .420 .120 .010 .760 .080 .570 .60 $0.190 .130 .500 .010 .56 \quad 0.270 .770 .39$ $\begin{array}{lllllllllll}0.05 & 0.06 & 0.23 & 0.94 & 0.75 & 0.83 & 0.23 & 0.45\end{array}$
Probability of green

\begin{tabular}{|llllll|l|l|}
\hline 0.6 & 0.6 & 0.6 & 0.6 & 0.6 & 0.6 & 0.7 & 0.7 \\
\hline 0.6 & 0.6 & 0.6 & 0.6 & 0.6 & 0.7 & 0.7 & 0.7 \\
0.6 & 0.6 & 0.6 & 0.6 & 0.7 & 0.7 & 0.7 & 0.7 \\
0.6 & 0.6 & 0.6 & 0.6 & 0.7 & 0.7 & 0.7 & 0.7 \\
0.6 & 0.6 & 0.6 & 0.7 & 0.7 & 0.7 & 0.7 & 0.7 \\
0.6 & 0.7 & 0.7 & 0.7 & 0.7 & 0.7 & 0.7 & 0.7 \\
\hline 0.7 & 0.7 & 0.7 & 0.7 & 0.7 & 0.7 & 0.7 & 0.7 \\
0.7 & 0.7 & 0.7 & 0.7 & 0.7 & 0.7 & 0.7 & 0.7 \\
0.7 & 0.7 & 0.7 & 0.7 & 0.7 & 0.7 & 0.7 & 0.7 \\
0.7 & 0.7 & 0.7 & 0.7 & 0.7 & 0.7 & 0.8 & 0.8 \\
0.7 & 0.7 & 0.7 & 0.7 & 0.7 & 0.8 & 0.8 & 0.8 \\
0.7 & 0.7 & 0.7 & 0.8 & 0.8 & 0.8 & 0.8 & 0.8 \\
0.7 & 0.8 & 0.8 & 0.8 & 0.8 & 0.8 & 0.8 & 0.9 \\
\hline 0.8 & 0.8 & 0.8 & 0.8 & 0.8 & 0.9 & 0.9 & 0.9 \\
0.8 & 0.8 & 0.8 & 0.8 & 0.9 & 0.9 & 0.9 & 0.9 \\
0.8 & 0.8 & 0.8 & 0.9 & 0.9 & 0.9 & 0.9 & 0.9 \\
0.8 & 0.8 & 0.9 & 0.9 & 0.9 & 0.9 & 0.9 & 0.9 \\
\hline
\end{tabular}

\section{Realization}

$\begin{array}{llllllll}0.6 & 0.6 & 0.6 & 0.6 & 0.6 & 0.6 & 0.7 & 0.7\end{array}$ $\begin{array}{llllllll}0.6 & 0.6 & 0.6 & 0.6 & 0.6 & 0.7 & 0.7 & 0.7\end{array}$ $\begin{array}{llllllll}0.6 & 0.6 & 0.6 & 0.6 & 0.7 & 0.7 & 0.7 & 0.7\end{array}$ $\begin{array}{lllllllll}0.6 & 0.6 & 0.6 & 0.6 & 0.7 & 0.7 & 0.7 & 0.7\end{array}$ $\begin{array}{lllllllll}0.6 & 0.6 & 0.6 & 0.7 & 0.7 & 0.7 & 0.7 & 0.7\end{array}$ $\begin{array}{lllllllll}0.6 & 0.7 & 0.7 & 0.7 & 0.7 & 0.7 & 0.7 & 0.7\end{array}$ $\begin{array}{llllllll}0.7 & 0.7 & 0.7 & 0.7 & 0.7 & 0.7 & 0.7 & 0.7\end{array}$ $\begin{array}{llllllll}0.7 & 0.7 & 0.7 & 0.7 & 0.7 & 0.7 & 0.7 & 0.7\end{array}$ $\begin{array}{llllllllll}0.7 & 0.7 & 0.7 & 0.7 & 0.7 & 0.7 & 0.7 & 0.7\end{array}$ $\begin{array}{llllllll}0.7 & 0.7 & 0.7 & 0.7 & 0.7 & 0.7 & 0.8 & 0.8\end{array}$ $\begin{array}{lllllllll}0.7 & 0.7 & 0.7 & 0.7 & 0.7 & 0.8 & 0.8 & 0.8\end{array}$ $\begin{array}{llllllll}0.7 & 0.7 & 0.7 & 0.8 & 0.8 & 0.8 & 0.8 & 0.8\end{array}$ $\begin{array}{llllllll}0.7 & 0.8 & 0.8 & 0.8 & 0.8 & 0.8 & 0.8 & 0.9\end{array}$ $\begin{array}{llllllll}0.8 & 0.8 & 0.8 & 0.8 & 0.8 & 0.9 & 0.9 & 0.9\end{array}$ $\begin{array}{lllllllll}0.8 & 0.8 & 0.8 & 0.8 & 0.9 & 0.9 & 0.9 & 0.9\end{array}$ $\begin{array}{llllllll}0.8 & 0.8 & 0.8 & 0.9 & 0.9 & 0.9 & 0.9 & 0.9\end{array}$ $\begin{array}{lllllllll}0.8 & 0.8 & 0.9 & 0.9 & 0.9 & 0.9 & 0.9 & 0.9\end{array}$

\begin{tabular}{lllllllll|}
0.6 & 0.6 & 0.6 & 0.6 & 0.6 & 0.6 & 0.7 & 0.7
\end{tabular} $\begin{array}{lllllllll}0.6 & 0.6 & 0.6 & 0.6 & 0.6 & 0.7 & 0.7 & 0.7\end{array}$ $\begin{array}{llllllll}0.6 & 0.6 & 0.6 & 0.6 & 0.7 & 0.7 & 0.7 & 0.7\end{array}$ $\begin{array}{llllllll}0.6 & 0.6 & 0.6 & 0.6 & 0.7 & 0.7 & 0.7 & 0.7\end{array}$ $\begin{array}{llllllll}0.6 & 0.6 & 0.6 & 0.7 & 0.7 & 0.7 & 0.7 & 0.7\end{array}$ $\begin{array}{lllllllll}0.6 & 0.7 & 0.7 & 0.7 & 0.7 & 0.7 & 0.7 & 0.7\end{array}$ $\begin{array}{llllllll}0.7 & 0.7 & 0.7 & 0.7 & 0.7 & 0.7 & 0.7 & 0.7\end{array}$ $\begin{array}{lllllllll}0.7 & 0.7 & 0.7 & 0.7 & 0.7 & 0.7 & 0.7 & 0.7\end{array}$ $\begin{array}{lllllllll}0.7 & 0.7 & 0.7 & 0.7 & 0.7 & 0.7 & 0.7 & 0.7\end{array}$ $\begin{array}{llllllll}0.7 & 0.7 & 0.7 & 0.7 & 0.7 & 0.7 & 0.8 & 0.8\end{array}$ $\begin{array}{llllllll}0.7 & 0.7 & 0.7 & 0.7 & 0.7 & 0.8 & 0.8 & 0.8\end{array}$ $\begin{array}{llllllll}0.7 & 0.7 & 0.7 & 0.8 & 0.8 & 0.8 & 0.8 & 0.8\end{array}$ $\begin{array}{llllllll}0.7 & 0.8 & 0.8 & 0.8 & 0.8 & 0.8 & 0.8 & 0.9\end{array}$ $\begin{array}{lllllllll}0.8 & 0.8 & 0.8 & 0.8 & 0.8 & 0.9 & 0.9 & 0.9\end{array}$ $\begin{array}{lllllllll}0.8 & 0.8 & 0.8 & 0.8 & 0.9 & 0.9 & 0.9 & 0.9\end{array}$ $\begin{array}{llllllll}0.8 & 0.8 & 0.8 & 0.9 & 0.9 & 0.9 & 0.9 & 0.9\end{array}$ $\begin{array}{llllllll}0.8 & 0.8 & 0.9 & 0.9 & 0.9 & 0.9 & 0.9 & 0.9\end{array}$
Field-based simulation Probability of green

Common PRN

\begin{tabular}{|lllllll|l|}
\hline 0.6 & 0.6 & 0.6 & 0.6 & 0.6 & 0.6 & 0.7 & 0.7 \\
0.6 & 0.6 & 0.6 & 0.6 & 0.6 & 0.7 & 0.7 & 0.7 \\
0.6 & 0.6 & 0.6 & 0.6 & 0.7 & 0.7 & 0.7 & 0.7 \\
0.6 & 0.6 & 0.6 & 0.6 & 0.7 & 0.7 & 0.7 & 0.7 \\
\hline 0.6 & 0.6 & 0.6 & 0.7 & 0.7 & 0.7 & 0.7 & 0.7 \\
0.6 & 0.7 & 0.7 & 0.7 & 0.7 & 0.7 & 0.7 & 0.7 \\
\hline 0.7 & 0.7 & 0.7 & 0.7 & 0.7 & 0.7 & 0.7 & 0.7 \\
0.7 & 0.7 & 0.7 & 0.7 & 0.7 & 0.7 & 0.7 & 0.7 \\
0.7 & 0.7 & 0.7 & 0.7 & 0.7 & 0.7 & 0.7 & 0.7 \\
\hline 0.7 & 0.7 & 0.7 & 0.7 & 0.7 & 0.7 & 0.8 & 0.8 \\
0.7 & 0.7 & 0.7 & 0.7 & 0.7 & 0.8 & 0.8 & 0.8 \\
0.7 & 0.7 & 0.7 & 0.8 & 0.8 & 0.8 & 0.8 & 0.8 \\
0.7 & 0.8 & 0.8 & 0.8 & 0.8 & 0.8 & 0.8 & 0.9 \\
\hline 0.8 & 0.8 & 0.8 & 0.8 & 0.8 & 0.9 & 0.9 & 0.9 \\
0.8 & 0.8 & 0.8 & 0.8 & 0.9 & 0.9 & 0.9 & 0.9 \\
0.8 & 0.8 & 0.8 & 0.9 & 0.9 & 0.9 & 0.9 & 0.9 \\
0.8 & 0.8 & 0.9 & 0.9 & 0.9 & 0.9 & 0.9 & 0.9 \\
\hline
\end{tabular}

\section{Realization}

$\begin{array}{llllllll}0.6 & 0.6 & 0.6 & 0.6 & 0.6 & 0.6 & 0.7 & 0.7\end{array}$ $\begin{array}{lllllllll}0.6 & 0.6 & 0.6 & 0.6 & 0.6 & 0.7 & 0.7 & 0.7\end{array}$ $\begin{array}{llllllll}0.6 & 0.6 & 0.6 & 0.6 & 0.7 & 0.7 & 0.7 & 0.7\end{array}$ $\begin{array}{lllllllll}0.6 & 0.6 & 0.6 & 0.6 & 0.7 & 0.7 & 0.7 & 0.7\end{array}$ $\begin{array}{llllllll}0.6 & 0.6 & 0.6 & 0.7 & 0.7 & 0.7 & 0.7 & 0.7\end{array}$ $\begin{array}{lllllllll}0.6 & 0.7 & 0.7 & 0.7 & 0.7 & 0.7 & 0.7 & 0.7\end{array}$ $\begin{array}{llllllll}0.7 & 0.7 & 0.7 & 0.7 & 0.7 & 0.7 & 0.7 & 0.7\end{array}$ $\begin{array}{llllllll}0.7 & 0.7 & 0.7 & 0.7 & 0.7 & 0.7 & 0.7 & 0.7\end{array}$ $\begin{array}{llllllll}0.7 & 0.7 & 0.7 & 0.7 & 0.7 & 0.7 & 0.7 & 0.7\end{array}$ $\begin{array}{lllllllll}0.7 & 0.7 & 0.7 & 0.7 & 0.7 & 0.7 & 0.8 & 0.8\end{array}$ $\begin{array}{lllllllll}0.7 & 0.7 & 0.7 & 0.7 & 0.7 & 0.8 & 0.8 & 0.8\end{array}$ $\begin{array}{llllllll}0.7 & 0.7 & 0.7 & 0.8 & 0.8 & 0.8 & 0.8 & 0.8\end{array}$ $\begin{array}{llllllll}0.7 & 0.8 & 0.8 & 0.8 & 0.8 & 0.8 & 0.8 & 0.9\end{array}$ $\begin{array}{llllllll}0.8 & 0.8 & 0.8 & 0.8 & 0.8 & 0.9 & 0.9 & 0.9\end{array}$ $\begin{array}{lllllllll}0.8 & 0.8 & 0.8 & 0.8 & 0.9 & 0.9 & 0.9 & 0.9\end{array}$ $\begin{array}{llllllll}0.8 & 0.8 & 0.8 & 0.9 & 0.9 & 0.9 & 0.9 & 0.9\end{array}$ $\begin{array}{llllllll}0.8 & 0.8 & 0.9 & 0.9 & 0.9 & 0.9 & 0.9 & 0.9\end{array}$

$\begin{array}{llllllll}0.6 & 0.6 & 0.6 & 0.6 & 0.6 & 0.6 & 0.7 & 0.7\end{array}$ $\begin{array}{lllllllll}0.6 & 0.6 & 0.6 & 0.6 & 0.6 & 0.7 & 0.7 & 0.7\end{array}$ $\begin{array}{llllllll}0.6 & 0.6 & 0.6 & 0.6 & 0.7 & 0.7 & 0.7 & 0.7\end{array}$ $\begin{array}{lllllllll}0.6 & 0.6 & 0.6 & 0.6 & 0.7 & 0.7 & 0.7 & 0.7\end{array}$ $\begin{array}{llllllll}0.6 & 0.6 & 0.6 & 0.7 & 0.7 & 0.7 & 0.7 & 0.7\end{array}$ $\begin{array}{lllllllll}0.6 & 0.7 & 0.7 & 0.7 & 0.7 & 0.7 & 0.7 & 0.7\end{array}$ $\begin{array}{llllllll}0.7 & 0.7 & 0.7 & 0.7 & 0.7 & 0.7 & 0.7 & 0.7\end{array}$ $\begin{array}{llllllll}0.7 & 0.7 & 0.7 & 0.7 & 0.7 & 0.7 & 0.7 & 0.7\end{array}$ $\begin{array}{lllllllll}0.7 & 0.7 & 0.7 & 0.7 & 0.7 & 0.7 & 0.7 & 0.7\end{array}$ $\begin{array}{llllllll}0.7 & 0.7 & 0.7 & 0.7 & 0.7 & 0.7 & 0.8 & 0.8\end{array}$ $\begin{array}{llllllll}0.7 & 0.7 & 0.7 & 0.7 & 0.7 & 0.8 & 0.8 & 0.8\end{array}$ $\begin{array}{lllllllll}0.7 & 0.7 & 0.7 & 0.8 & 0.8 & 0.8 & 0.8 & 0.8\end{array}$ $\begin{array}{lllllllll}0.7 & 0.8 & 0.8 & 0.8 & 0.8 & 0.8 & 0.8 & 0.9\end{array}$ $\begin{array}{llllllll}0.8 & 0.8 & 0.8 & 0.8 & 0.8 & 0.9 & 0.9 & 0.9\end{array}$ $\begin{array}{lllllllll}0.8 & 0.8 & 0.8 & 0.8 & 0.9 & 0.9 & 0.9 & 0.9\end{array}$ $\begin{array}{llllllll}0.8 & 0.8 & 0.8 & 0.9 & 0.9 & 0.9 & 0.9 & 0.9\end{array}$ $\begin{array}{llllllll}0.8 & 0.8 & 0.9 & 0.9 & 0.9 & 0.9 & 0.9 & 0.9\end{array}$
Common PRN

0.30

0.51

Figure continued on next page... 
Independent pixel-based simulation Independent PRNs

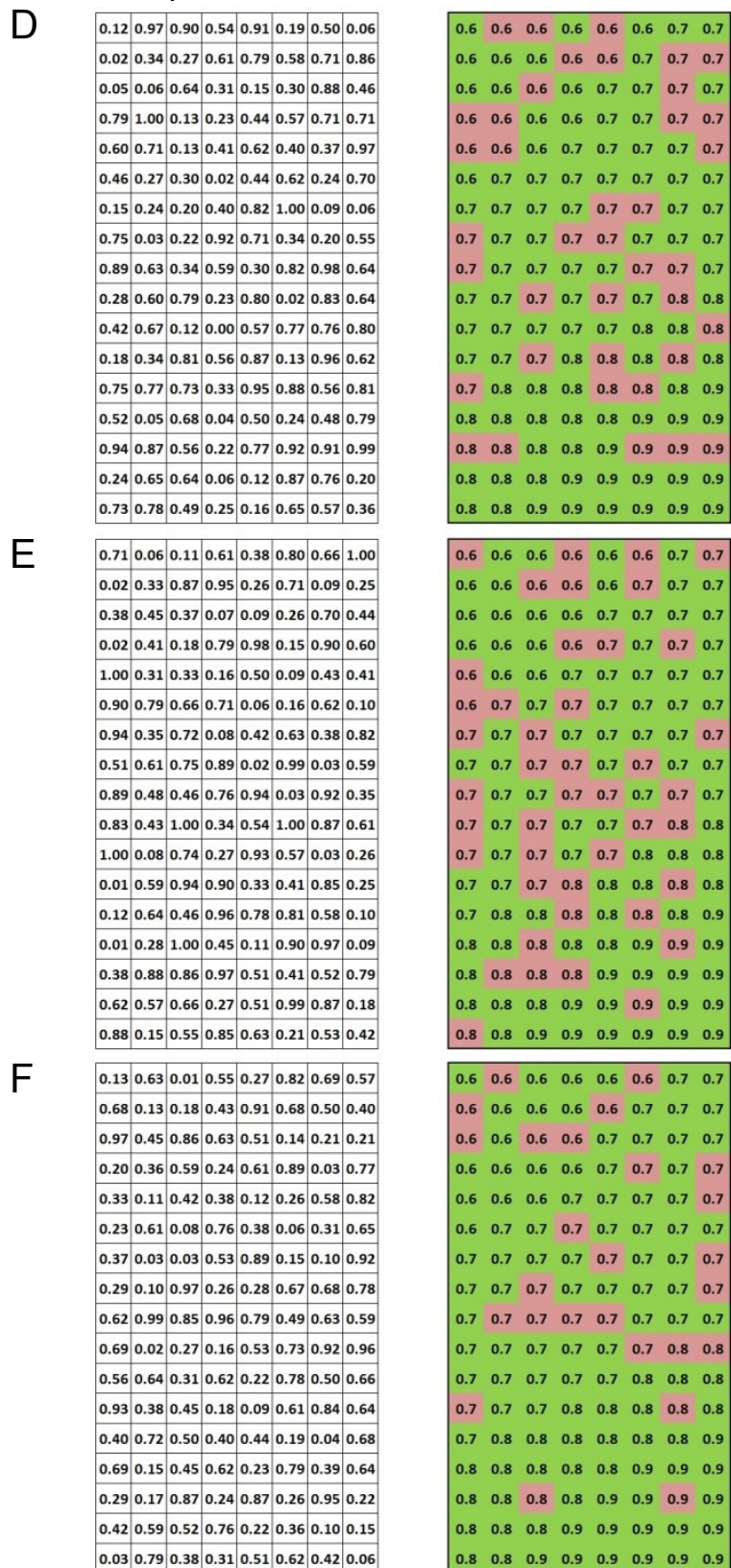

Field-based simulation Realization

\begin{tabular}{|llllllll|}
\hline 0.6 & 0.6 & 0.6 & 0.6 & 0.6 & 0.6 & 0.7 & 0.7 \\
0.6 & 0.6 & 0.6 & 0.6 & 0.6 & 0.7 & 0.7 & 0.7 \\
0.6 & 0.6 & 0.6 & 0.6 & 0.7 & 0.7 & 0.7 & 0.7 \\
0.6 & 0.6 & 0.6 & 0.6 & 0.7 & 0.7 & 0.7 & 0.7 \\
0.6 & 0.6 & 0.6 & 0.7 & 0.7 & 0.7 & 0.7 & 0.7 \\
0.6 & 0.7 & 0.7 & 0.7 & 0.7 & 0.7 & 0.7 & 0.7 \\
0.7 & 0.7 & 0.7 & 0.7 & 0.7 & 0.7 & 0.7 & 0.7 \\
0.7 & 0.7 & 0.7 & 0.7 & 0.7 & 0.7 & 0.7 & 0.7 \\
0.7 & 0.7 & 0.7 & 0.7 & 0.7 & 0.7 & 0.7 & 0.7 \\
0.7 & 0.7 & 0.7 & 0.7 & 0.7 & 0.7 & 0.8 & 0.8 \\
0.7 & 0.7 & 0.7 & 0.7 & 0.7 & 0.8 & 0.8 & 0.8 \\
0.7 & 0.7 & 0.7 & 0.8 & 0.8 & 0.8 & 0.8 & 0.8 \\
0.7 & 0.8 & 0.8 & 0.8 & 0.8 & 0.8 & 0.8 & 0.9 \\
0.8 & 0.8 & 0.8 & 0.8 & 0.8 & 0.9 & 0.9 & 0.9 \\
0.8 & 0.8 & 0.8 & 0.8 & 0.9 & 0.9 & 0.9 & 0.9 \\
0.8 & 0.8 & 0.8 & 0.9 & 0.9 & 0.9 & 0.9 & 0.9 \\
0.8 & 0.8 & 0.9 & 0.9 & 0.9 & 0.9 & 0.9 & 0.9 \\
\hline & & & & & & & \\
\hline
\end{tabular}

\begin{tabular}{|llllllll|}
\hline 0.6 & 0.6 & 0.6 & 0.6 & 0.6 & 0.6 & 0.7 & 0.7
\end{tabular} $\begin{array}{llllllll}0.6 & 0.6 & 0.6 & 0.6 & 0.6 & 0.7 & 0.7 & 0.7\end{array}$ $\begin{array}{lllllllll}0.6 & 0.6 & 0.6 & 0.6 & 0.7 & 0.7 & 0.7 & 0.7\end{array}$ $\begin{array}{llllllll}0.6 & 0.6 & 0.6 & 0.6 & 0.7 & 0.7 & 0.7 & 0.7\end{array}$ $\begin{array}{llllllll}0.6 & 0.6 & 0.6 & 0.7 & 0.7 & 0.7 & 0.7 & 0.7\end{array}$ $\begin{array}{llllllll}0.6 & 0.7 & 0.7 & 0.7 & 0.7 & 0.7 & 0.7 & 0.7\end{array}$ $\begin{array}{llllllll}0.7 & 0.7 & 0.7 & 0.7 & 0.7 & 0.7 & 0.7 & 0.7\end{array}$ $\begin{array}{llllllll}0.7 & 0.7 & 0.7 & 0.7 & 0.7 & 0.7 & 0.7 & 0.7\end{array}$ $\begin{array}{llllllll}0.7 & 0.7 & 0.7 & 0.7 & 0.7 & 0.7 & 0.7 & 0.7\end{array}$ $\begin{array}{llllllll}0.7 & 0.7 & 0.7 & 0.7 & 0.7 & 0.7 & 0.8 & 0.8\end{array}$ $\begin{array}{llllllll}0.7 & 0.7 & 0.7 & 0.7 & 0.7 & 0.8 & 0.8 & 0.8\end{array}$ $\begin{array}{lllllllll}0.7 & 0.7 & 0.7 & 0.8 & 0.8 & 0.8 & 0.8 & 0.8\end{array}$ $\begin{array}{llllllll}0.7 & 0.8 & 0.8 & 0.8 & 0.8 & 0.8 & 0.8 & 0.9\end{array}$ $\begin{array}{llllllll}0.8 & 0.8 & 0.8 & 0.8 & 0.8 & 0.9 & 0.9 & 0.9\end{array}$ $\begin{array}{llllllll}0.8 & 0.8 & 0.8 & 0.8 & 0.9 & 0.9 & 0.9 & 0.9\end{array}$ $\begin{array}{lllllllll}0.8 & 0.8 & 0.8 & 0.9 & 0.9 & 0.9 & 0.9 & 0.9\end{array}$ $\begin{array}{lllllllll}0.8 & 0.8 & 0.9 & 0.9 & 0.9 & 0.9 & 0.9 & 0.9\end{array}$

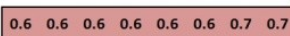

$\begin{array}{llllllll}0.6 & 0.6 & 0.6 & 0.6 & 0.6 & 0.7 & 0.7 & 0.7\end{array}$

$\begin{array}{llllllll}0.6 & 0.6 & 0.6 & 0.6 & 0.7 & 0.7 & 0.7 & 0.7\end{array}$ $\begin{array}{llllllll}0.6 & 0.6 & 0.6 & 0.6 & 0.7 & 0.7 & 0.7 & 0.7\end{array}$ $\begin{array}{llllllll}0.6 & 0.6 & 0.6 & 0.7 & 0.7 & 0.7 & 0.7 & 0.7\end{array}$ $\begin{array}{llllllll}0.6 & 0.7 & 0.7 & 0.7 & 0.7 & 0.7 & 0.7 & 0.7\end{array}$ $\begin{array}{lllllllll}0.7 & 0.7 & 0.7 & 0.7 & 0.7 & 0.7 & 0.7 & 0.7\end{array}$ $\begin{array}{lllllllll}0.7 & 0.7 & 0.7 & 0.7 & 0.7 & 0.7 & 0.7 & 0.7\end{array}$ $\begin{array}{llllllll}0.7 & 0.7 & 0.7 & 0.7 & 0.7 & 0.7 & 0.7 & 0.7\end{array}$ $\begin{array}{llllllll}0.7 & 0.7 & 0.7 & 0.7 & 0.7 & 0.7 & 0.8 & 0.8\end{array}$ $\begin{array}{lllllllll}0.7 & 0.7 & 0.7 & 0.7 & 0.7 & 0.8 & 0.8 & 0.8\end{array}$ $\begin{array}{lllllllll}0.7 & 0.7 & 0.7 & 0.8 & 0.8 & 0.8 & 0.8 & 0.8\end{array}$ $\begin{array}{llllllll}0.7 & 0.8 & 0.8 & 0.8 & 0.8 & 0.8 & 0.8 & 0.9\end{array}$ $\begin{array}{llllllll}0.8 & 0.8 & 0.8 & 0.8 & 0.8 & 0.9 & 0.9 & 0.9\end{array}$ $\begin{array}{llllllll}0.8 & 0.8 & 0.8 & 0.8 & 0.9 & 0.9 & 0.9 & 0.9\end{array}$ $\begin{array}{llllllll}0.8 & 0.8 & 0.8 & 0.9 & 0.9 & 0.9 & 0.9 & 0.9\end{array}$ $\begin{array}{lllllllll}0.8 & 0.8 & 0.9 & 0.9 & 0.9 & 0.9 & 0.9 & 0.9\end{array}$

\section{Common PRN}

0.88

0.25

Figure A.1 (continued from page A1) Examples of realizations of thematic class

(green or red) under independent pixel-based and field-based simulation. Each rectangle represents a field (containing several pixels), wherein green is the mostlikely class. Shown are (A) the probability of green occurring for any pixel within the field (as opposed to red), and (B, C, D, E, F) five realizations of thematic class for the full field, under each simulation approach. 
Appendix B: Spectral characteristics of the training data for the limited extent classifications
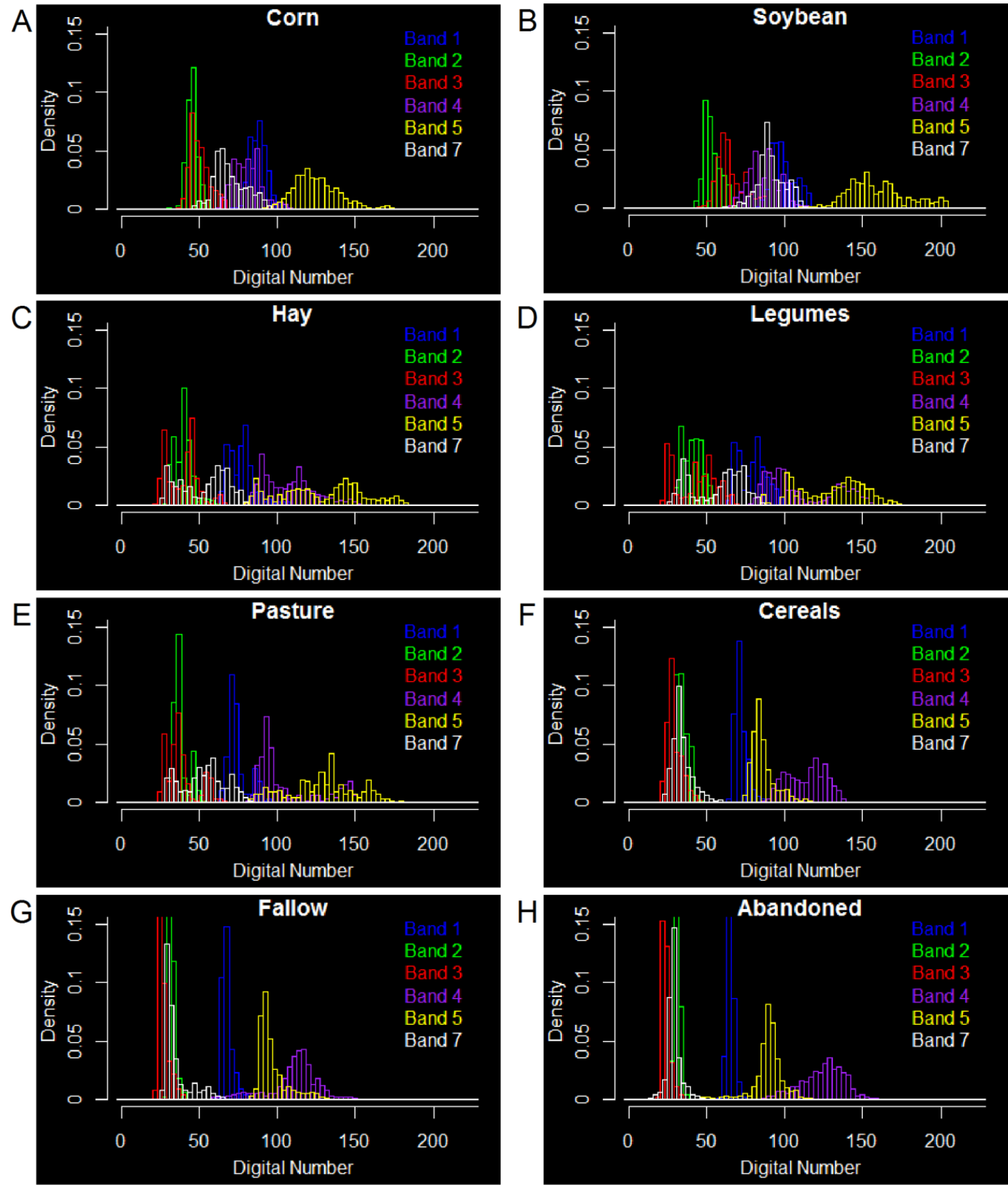

Figure B.1 Training data values, by class and spectral band, for the 19 June

Thematic Mapper image (limited classification extent). See Sections 3.3.4 and 3.3.6. 

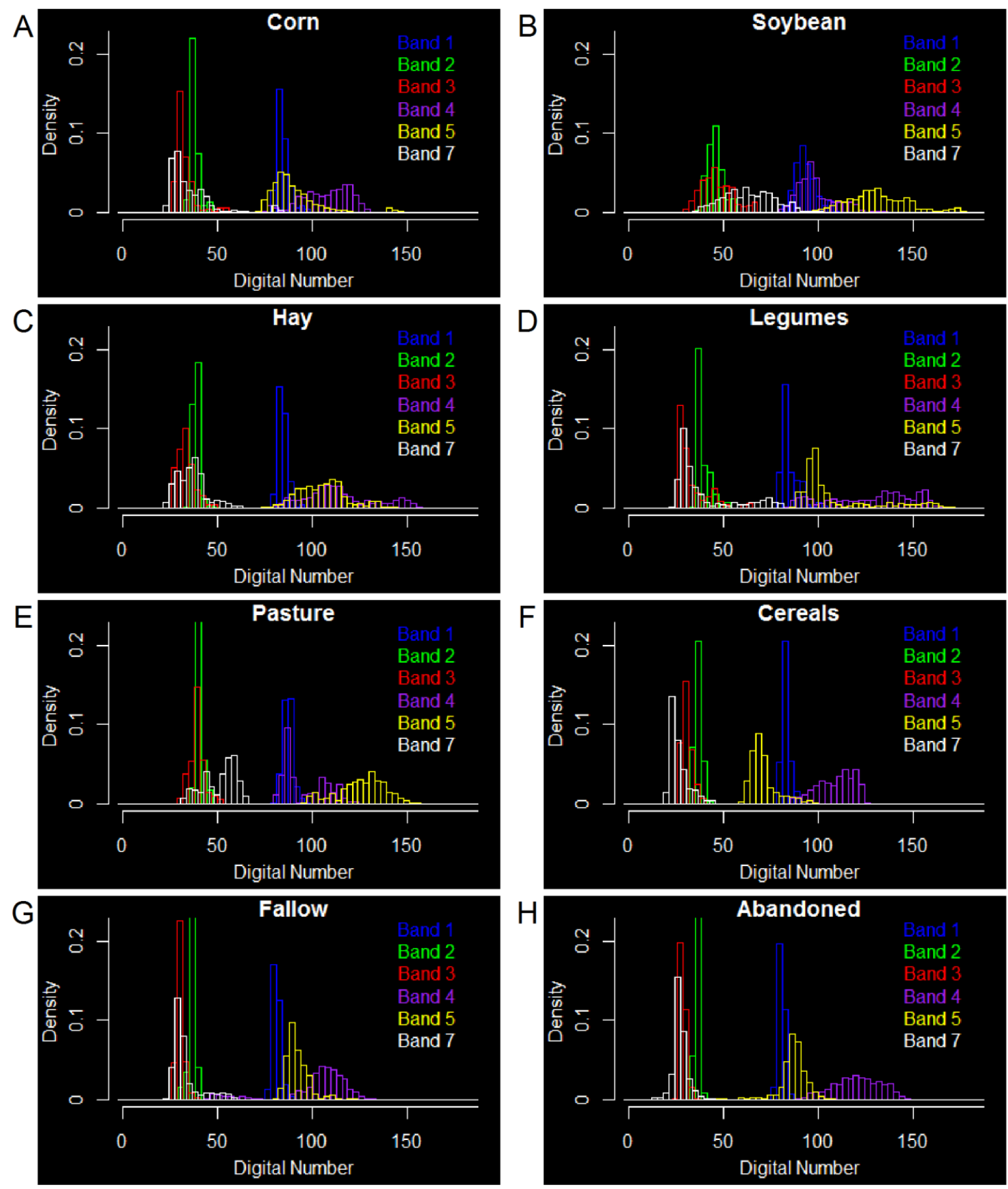

Figure B.2 Training data values, by class and spectral band, for the 5 July

Thematic Mapper image (limited classification extent). See Sections 3.3.4 and 3.3.6. 

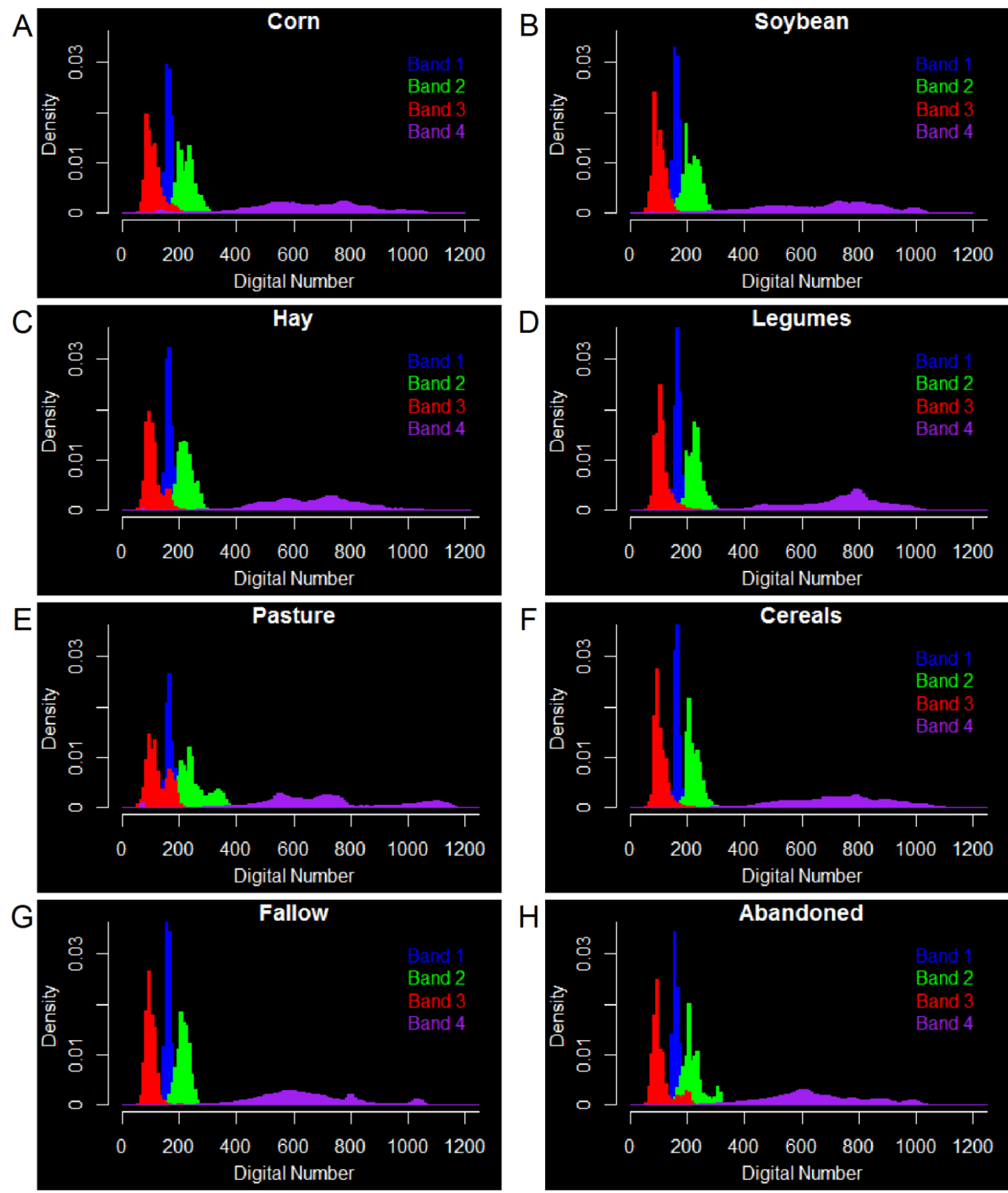

Figure B.3 Training data values, by class and spectral band, for the 29 August

QuickBird image. See Sections 3.3.4 and 3.3.6. 
Appendix C: Transformed divergence for the limited extent classifications

Transformed divergence is a commonly used measure of spectral separability between two classes, as defined for the training data (Kavzoglu \& Mather 2002; Lillesand et al. 2004). These values range from 0 (identical) to 2000 (maximum seprarbility) (Lillesand et al. 2004). Tso \& Mather (2009, p. 53) define the equation for the divergence (D) and the transformed divergence (TD) between classes $i$ and $j$, as:

$$
\begin{aligned}
& \mathrm{D}_{i j}=\frac{\operatorname{tr}\left(\left(C_{i}-C_{j}\right) \times\left(C_{j}^{-1}-C_{i}^{-1}\right)\right)}{2} \\
&+\frac{\operatorname{tr}\left(\left(C_{i}^{-1}+C_{j}^{-1}\right) \times\left(\mu_{i}-\mu_{j}\right) \times\left(\mu_{i}-\mu_{j}\right)^{T}\right)}{2} \\
& \mathrm{TD}_{i j}=2000 \times\left(1-e^{\frac{\mathrm{D}_{i j}}{8}}\right)
\end{aligned}
$$

where $C$ is the variance-covariance matrix for the cluster, $\mu$ is the cluster mean, and $t r$ and $T$ are the trace and transpose of a matrix, respectively. Lillesand et al. (2004) suggest that TD values less than 1500 represent spectrally similar classes, whereas Kavzoglu \& Mather (2002) cite Jensen (1996), suggesting that values less than 1700 represent poor separability. 
Table C.1 Transformed divergence values, by class, for the single date TM image set. Class pairs with values less than 1700 are highlighted in yellow as having poor separability. The mean transformed divergence value, across all class pairs was 1856.

\begin{tabular}{|r|c|c|c|c|c|c|c|c|}
\hline & Corn & Soybean & Hay & Pasture & Legumes & Cereals & Fallow & Abandoned \\
\hline Corn & & & & & & & & \\
\hline Soybean & 1388 & & & & & & & \\
\hline Hay & 1985 & 1999 & & & & & & \\
\hline Pasture & 2000 & 2000 & 1083 & & & & & \\
\hline Legumes & 1972 & 1999 & 1059 & 1418 & & & & \\
\hline Cereals & 1999 & 2000 & 1996 & 2000 & 1995 & & & \\
\hline Fallow & 2000 & 2000 & 1977 & 1962 & 1995 & 1952 & & \\
\hline Abandoned & 2000 & 2000 & 1989 & 1983 & 2000 & 1924 & 1287 & \\
\hline
\end{tabular}

Table C.2 Transformed divergence values, by class, for the multi-date TM image set. No class pairs had values less than 1700. The mean transformed divergence value, across all class pairs was 1988 .

\begin{tabular}{|r|r|r|r|r|r|r|r|l|}
\hline & Corn & Soybean & Hay & Pasture & Legumes & Cereals & Fallow & Abandoned \\
\hline Corn & & & & & & & & \\
\hline Soybean & 1950 & & & & & & & \\
\hline Hay & 2000 & 2000 & & & & & & \\
\hline Pasture & 2000 & 2000 & 1999 & & & & & \\
\hline Legumes & 2000 & 2000 & 1900 & 2000 & & & & \\
\hline Cereals & 2000 & 2000 & 2000 & 2000 & 2000 & & & \\
\hline Fallow & 2000 & 2000 & 2000 & 2000 & 2000 & 2000 & & \\
\hline Abandoned & 2000 & 2000 & 2000 & 2000 & 2000 & 2000 & 1822 & \\
\hline
\end{tabular}

Table C.3 Transformed divergence values, by class, for the single date QB image set. Class pairs with values less than 1700 are highlighted in yellow as having poor separability. The mean transformed divergence value, across all class pairs was 1575 .

\begin{tabular}{|r|c|c|c|c|c|c|c|c|}
\hline & Corn & Soybean & Hay & Pasture & Legumes & Cereals & Fallow & Abandoned \\
\hline Corn & & & & & & & & \\
\hline Soybean & 945 & & & & & & & \\
\hline Hay & 1482 & 1645 & & & & & & \\
\hline Pasture & 1283 & 1851 & 713 & & & & & \\
\hline Legumes & 1500 & 1515 & 497 & 1374 & & & & \\
\hline Cereals & 2000 & 2000 & 1996 & 1998 & 2000 & & & \\
\hline Fallow & 1912 & 1984 & 1474 & 1212 & 1904 & 1999 & & \\
\hline Abandond & 1957 & 1982 & 1100 & 1207 & 1791 & 1856 & 913 & \\
\hline
\end{tabular}




\section{Appendix D: Examples of error and uncertainty in the reference field}

boundaries

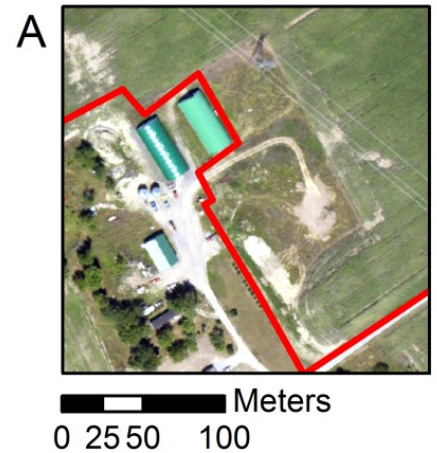

Portion of farmyard included in field. Alternate boundary involved a split and removal of farmyard portion from consideration.
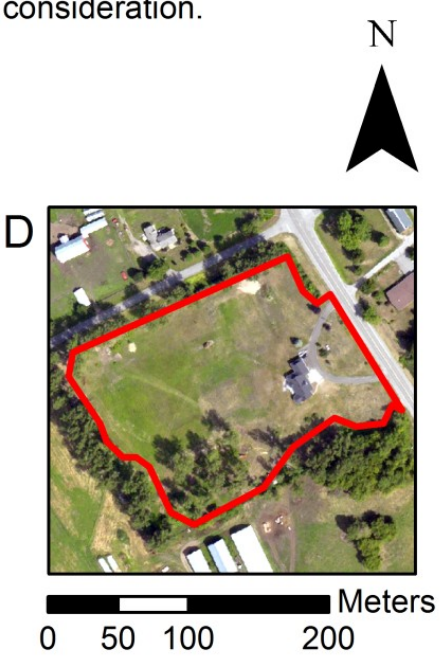

Field referenced as 'pasture'. House, driveway, lawn, and trees are included in field. Alternate involved removal of residence and treed portion from the field.
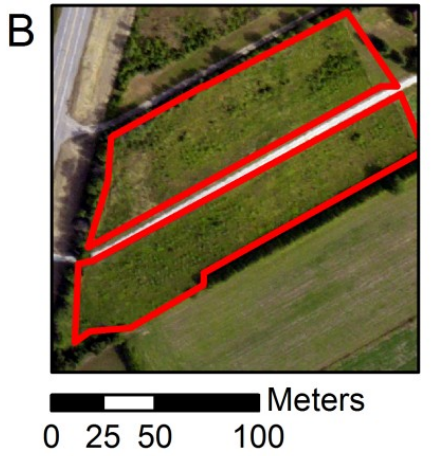

Refernce fields had 'unknown' crop type, suggesting that they may have been missed during referencing. Fields appear abandoned in air photo. Alternate boundary involved removal of fields from consideration.

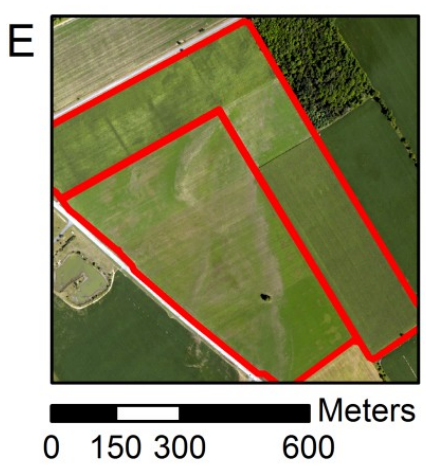

These two fields had multiple alternate possible representations involving combinations of splits and merges.

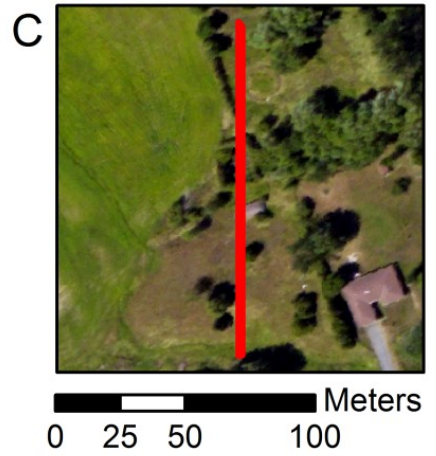

A very rare case. Sliver polygon listed as 'field' of type 'unknown'. Would not have influenced SHDI but could have significant influence over MFS. Alternate involved removal from consideration.

F

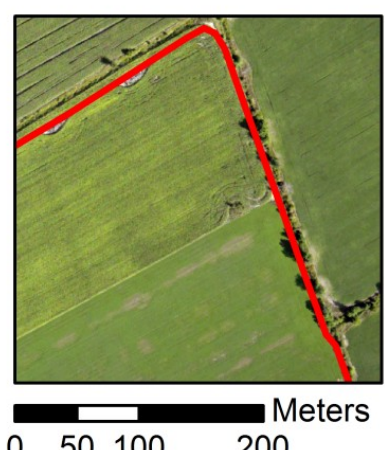

This field was referenced as 'legumes' but the northern portion appears to be corn. Alternate involved a split.

Figure D.1 Examples of error and uncertainty in the reference field boundaries.

Boundaries of the field or fields in question are shown in red. The cases shown are not typical boundary interpretation uncertainty cases, but were selected to demonstrate particular instances of error and uncertainty in the reference boundaries. 
Appendix E: Spectral characteristics and spatial distribution of the training samples for the 2011 study area crop classification
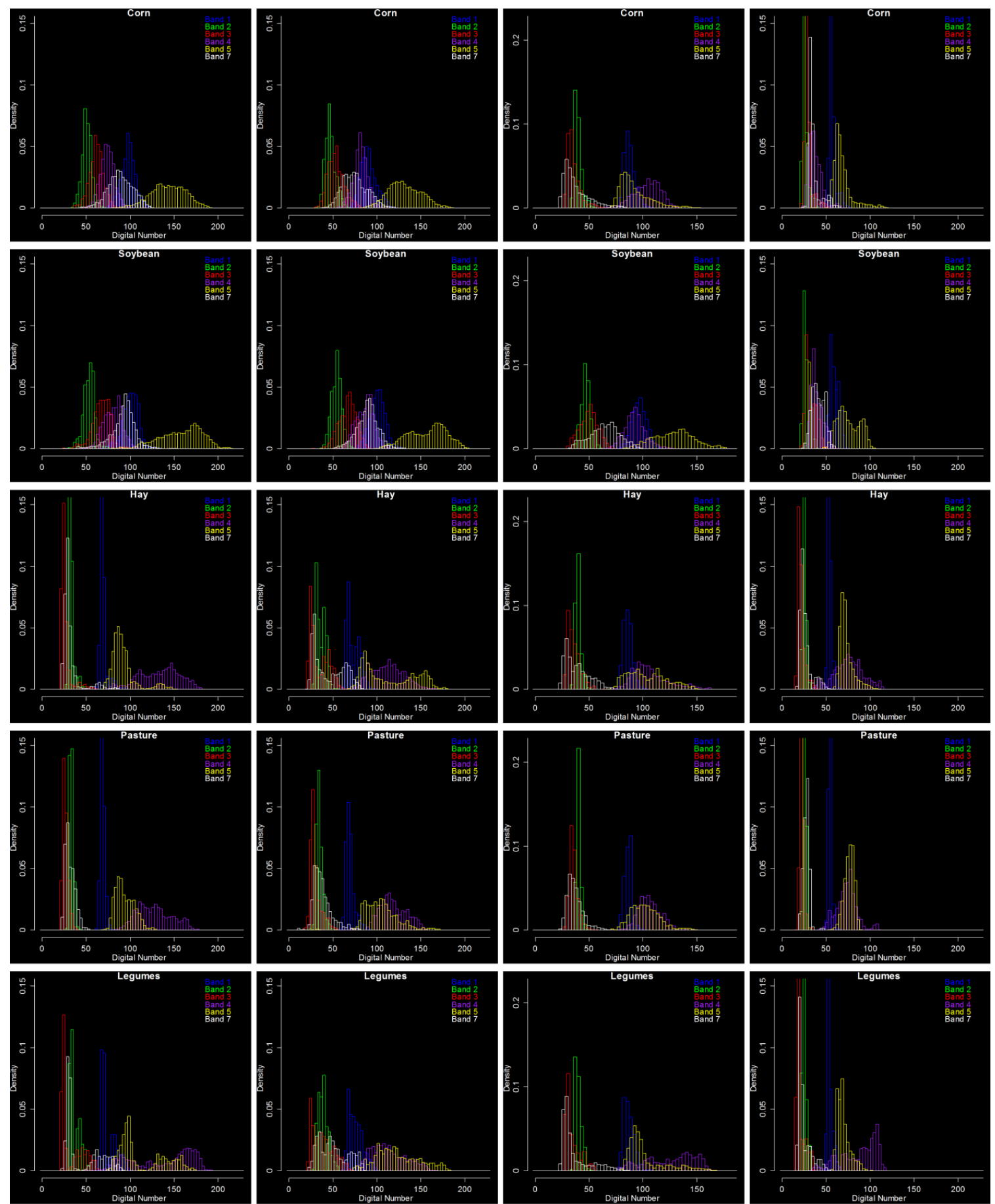

政

3 June, TM Image

19 June, TM Image

5 July, TM Image

9 October, TM Image

Figure continued on next page... 

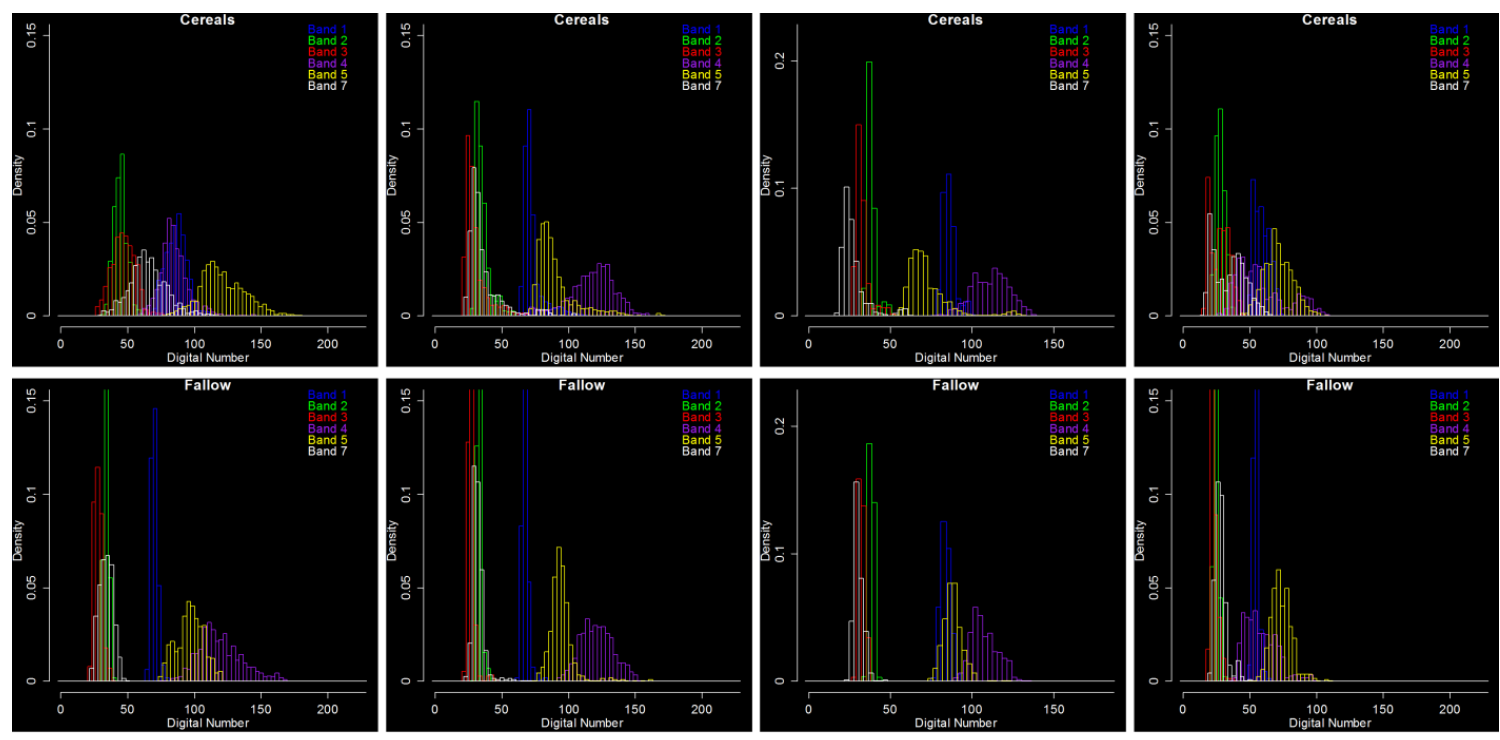

3 June, TM Image 19 June, TM Image

5 July, TM Image 9 October, TM Image

Figure E.1 (continued from page E1) Spectral characteristics, by class and

Thematic Mapper (TM) image date, for the training data of the 2011 full study area crop classification. 

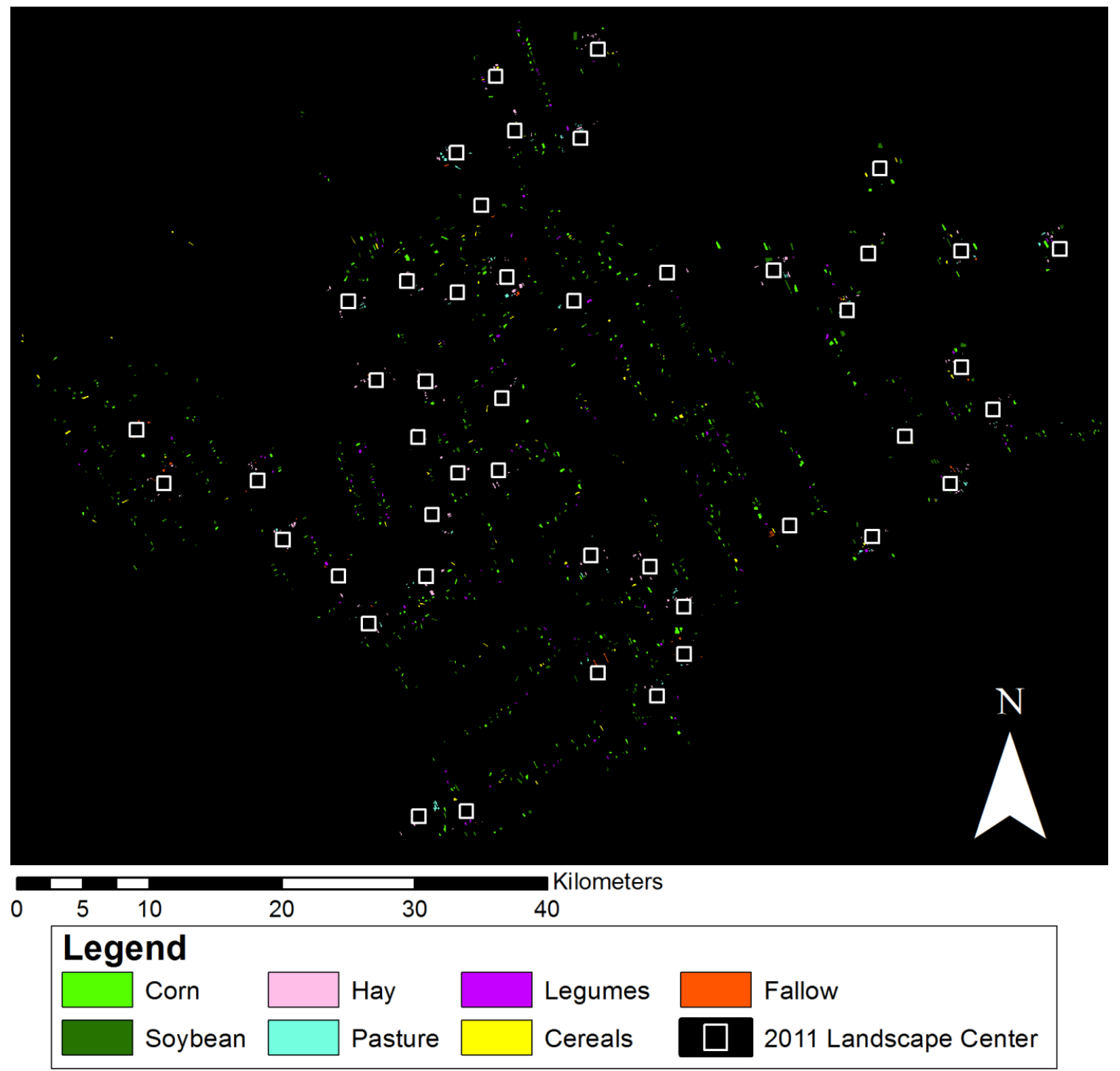

Figure E.2 Spatial distribution of training samples for the 2011 full study area crop classification. 
Appendix F: Additional classification entropy and accuracy information [limited extent classifications]

Table F.1 Mean normalized entropy $\left(E_{\mathrm{n}}\right)$ values for the correctly and incorrectly classified areas of the field-aggregated classifications.

\begin{tabular}{|c|c|c|c|c|c|}
\hline \multirow{2}{*}{$\begin{array}{c}\text { Image } \\
\text { Set }\end{array}$} & \multirow{2}{*}{$\begin{array}{l}\text { Correctly vs. } \\
\text { Incorrectly } \\
\text { Classified } \\
\text { Area }\end{array}$} & \multicolumn{4}{|c|}{ Mean Normalized Entropy } \\
\hline & & $\begin{array}{c}\text { Landscape } \\
\text { A }\end{array}$ & $\begin{array}{c}\text { Landscape } \\
\text { B }\end{array}$ & $\begin{array}{c}\text { Landscape } \\
\text { C }\end{array}$ & $\begin{array}{c}\text { Landscape } \\
\text { D }\end{array}$ \\
\hline \multirow{2}{*}{$\begin{array}{c}\text { Single } \\
\text { date TM }\end{array}$} & Correctly & 0.287 & 0.304 & 0.388 & 0.261 \\
\hline & Incorrectly & 0.420 & 0.486 & 0.508 & 0.228 \\
\hline \multirow{2}{*}{$\begin{array}{c}\text { Multi-date } \\
\text { TM }\end{array}$} & Correctly & 0.141 & 0.203 & 0.191 & 0.144 \\
\hline & Incorrectly & 0.275 & 0.319 & 0.271 & 0.239 \\
\hline \multirow{2}{*}{ QB } & Correctly & 0.454 & 0.655 & 0.632 & 0.598 \\
\hline & Incorrectly & 0.720 & 0.699 & 0.761 & 0.645 \\
\hline
\end{tabular}




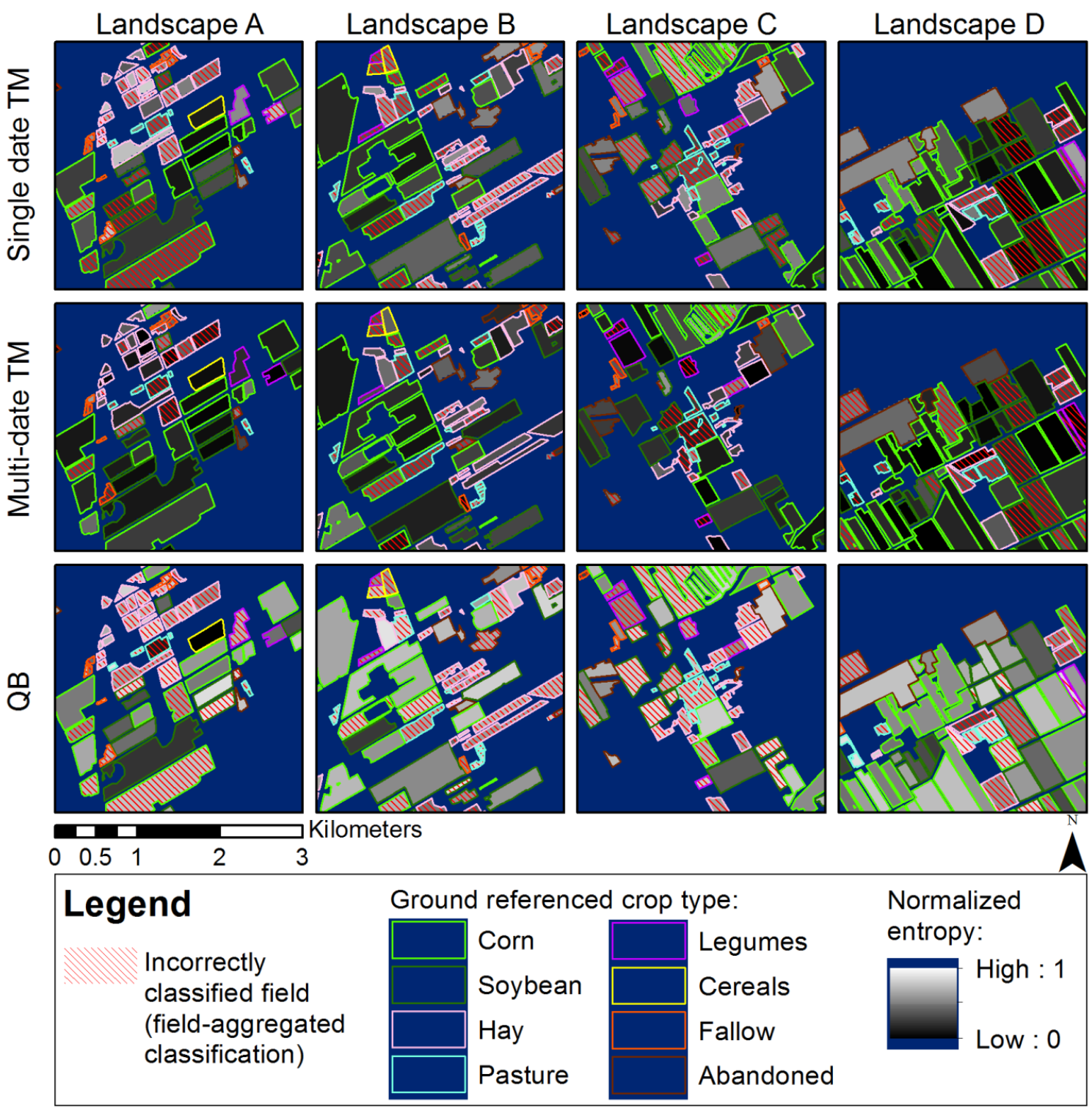

Figure F.1 Normalized entropy $\left(E_{\mathrm{n}}\right)$ values of field-aggregated probabilities of class membership, as well as incorrectly classified fields in the hardened classification. 
Table F.2 Error matrix for the per-pixel single date TM classification of Landscape A.

\begin{tabular}{|r|c|c|c|c|c|c|c|c|c|}
\hline & Corn & Soy. & Hay & Pasture & Legumes & Cereals & Fallow & Abandon & $\begin{array}{c}\text { User's } \\
\text { Acc. (\%) }\end{array}$ \\
\hline Corn & 1040 & 328 & 42 & 44 & 10 & 2 & 7 & 0 & 70.6 \\
\hline Soybean & 403 & 580 & 39 & 8 & 0 & 0 & 1 & 0 & 56.3 \\
\hline Hay & 0 & 0 & 183 & 9 & 16 & 0 & 2 & 0 & 87.1 \\
\hline Pasture & 0 & 26 & 61 & 8 & 4 & 0 & 4 & 4 & 7.5 \\
\hline Legumes & 35 & 10 & 138 & 5 & 62 & 0 & 15 & 1 & 23.3 \\
\hline Cereals & 26 & 0 & 32 & 6 & 4 & 90 & 25 & 6 & 47.6 \\
\hline Fallow & 2 & 0 & 70 & 2 & 0 & 0 & 1 & 7 & 1.2 \\
\hline Abandon. & 0 & 0 & 47 & 3 & 23 & 0 & 19 & 17 & 15.6 \\
\hline $\begin{array}{r}\text { Producer's } \\
\text { Acc. (\%) }\end{array}$ & 69.1 & 61.4 & 29.9 & 9.4 & 52.1 & 97.8 & 1.4 & 48.6 & $\begin{array}{c}\text { Overall: } \\
57.1 \%\end{array}$ \\
\hline
\end{tabular}

Table F.3 Error matrix for the per-pixel single date TM classification of Landscape B.

\begin{tabular}{|r|c|c|c|c|c|c|c|c|c|}
\hline & Corn & Soy. & Hay & Pasture & Legumes & Cereals & Fallow & Abandon & $\begin{array}{c}\text { User's } \\
\text { Acc. (\%) }\end{array}$ \\
\hline Corn & 1327 & 162 & 3 & 1 & 0 & 1 & 1 & 1 & 88.7 \\
\hline Soybean & 161 & 765 & 0 & 0 & 0 & 0 & 0 & 1 & 82.5 \\
\hline Hay & 21 & 5 & 237 & 74 & 32 & 14 & 14 & 7 & 58.7 \\
\hline Pasture & 0 & 0 & 86 & 1 & 0 & 0 & 3 & 7 & 1.0 \\
\hline Legumes & 94 & 76 & 83 & 94 & 7 & 45 & 0 & 3 & 1.7 \\
\hline Cereals & 7 & 0 & 39 & 7 & 0 & 10 & 1 & 29 & 10.8 \\
\hline Fallow & 7 & 0 & 85 & 14 & 0 & 0 & 26 & 39 & 15.2 \\
\hline Abandon. & 0 & 0 & 239 & 28 & 0 & 0 & 9 & 149 & 35.1 \\
\hline $\begin{array}{r}\text { Producer's } \\
\text { Acc. (\%) }\end{array}$ & 82.1 & 75.9 & 30.7 & 0.5 & 17.9 & 14.3 & 48.1 & 63.1 & $\begin{array}{c}\text { Overall: } \\
62.8 \%\end{array}$ \\
\hline
\end{tabular}

Table F.4 Error matrix for the per-pixel single date TM classification of Landscape C.

\begin{tabular}{|r|c|c|c|c|c|c|c|c|c|}
\hline & Corn & Soy. & Hay & Pasture & Legumes & Cereals & Fallow & Abandon & $\begin{array}{c}\text { User's } \\
\text { Acc. (\%) }\end{array}$ \\
\hline Corn & 697 & 159 & 2 & 0 & 0 & 0 & 0 & 0 & 81.2 \\
\hline Soybean & 162 & 504 & 0 & 1 & 0 & 0 & 0 & 0 & 75.6 \\
\hline Hay & 60 & 34 & 166 & 163 & 63 & 0 & 1 & 3 & 33.9 \\
\hline Pasture & 4 & 4 & 20 & 0 & 8 & 0 & 2 & 21 & 0 \\
\hline Legumes & 132 & 238 & 53 & 15 & 62 & 0 & 7 & 9 & 12.0 \\
\hline Cereals & 10 & 2 & 21 & 0 & 1 & 0 & 6 & 33 & 0 \\
\hline Fallow & 69 & 0 & 56 & 27 & 0 & 0 & 21 & 24 & 10.7 \\
\hline Abandon. & 0 & 0 & 48 & 1 & 124 & 0 & 11 & 201 & 52.2 \\
\hline $\begin{array}{r}\text { Producer's } \\
\text { Acc. (\%) }\end{array}$ & 61.5 & 53.6 & 45.4 & 0 & 24.0 & 0 & 43.8 & 69.1 & $\begin{array}{c}\text { Overall: } \\
50.9 \%\end{array}$ \\
\hline
\end{tabular}

Table F.5 Error matrix for the per-pixel single date TM classification of Landscape D.

\begin{tabular}{|r|c|c|c|c|c|c|c|c|c|}
\hline & Corn & Soy. & Hay & Pasture & Legumes & Cereals & Fallow & Abandon & $\begin{array}{c}\text { User's } \\
\text { Acc. (\%) }\end{array}$ \\
\hline Corn & 2066 & 823 & 92 & 1 & 35 & 0 & 0 & 1 & 68.5 \\
\hline Soybean & 359 & 588 & 38 & 0 & 4 & 0 & 0 & 0 & 59.5 \\
\hline Hay & 4 & 4 & 75 & 81 & 14 & 0 & 0 & 25 & 36.9 \\
\hline Pasture & 2 & 1 & 49 & 34 & 8 & 0 & 0 & 8 & 33.3 \\
\hline Legumes & 65 & 38 & 104 & 7 & 52 & 0 & 0 & 9 & 18.9 \\
\hline Cereals & 3 & 1 & 2 & 1 & 0 & 0 & 0 & 30 & 0 \\
\hline Fallow & 2 & 0 & 0 & 17 & 3 & 0 & 3 & 91 & 2.6 \\
\hline Abandon. & 0 & 0 & 0 & 13 & 2 & 0 & 2 & 504 & 96.7 \\
\hline $\begin{array}{r}\text { Producer's } \\
\text { Acc. (\%) }\end{array}$ & 82.6 & 40.4 & 20.8 & 22.1 & 44.1 & 0 & 60.0 & 75.4 & $\begin{array}{c}\text { Overall: } \\
63.1 \%\end{array}$ \\
\hline
\end{tabular}


Table F.6 Error matrix for the per-field single date TM classification of Landscape A.

\begin{tabular}{|r|c|c|c|c|c|c|c|c|c|}
\hline & Corn & Soy. & Hay & Pasture & Legumes & Cereals & Fallow & Aband. & $\begin{array}{c}\text { User's } \\
\text { Acc. (\%) }\end{array}$ \\
\hline Corn & 896 & 209 & 0 & 54 & 0 & 0 & 14 & 0 & 76.4 \\
\hline Soybean & 610 & 705 & 68 & 0 & 0 & 0 & 0 & 0 & 51.0 \\
\hline Hay & 0 & 0 & 293 & 8 & 0 & 0 & 0 & 0 & 97.3 \\
\hline Pasture & 0 & 30 & 13 & 10 & 0 & 0 & 0 & 0 & 18.9 \\
\hline Legumes & 0 & 0 & 106 & 0 & 75 & 0 & 28 & 0 & 35.9 \\
\hline Cereals & 0 & 0 & 37 & 7 & 0 & 92 & 26 & 11 & 53.2 \\
\hline Fallow & 0 & 0 & 33 & 0 & 0 & 0 & 0 & 4 & 0 \\
\hline Abandon. & 0 & 0 & 62 & 6 & 44 & 0 & 6 & 20 & 14.5 \\
\hline $\begin{array}{r}\text { Producer's } \\
\text { Acc. (\%) }\end{array}$ & 59.5 & 74.7 & 47.9 & 11.8 & 63.0 & 100 & 0 & 57.1 & $\begin{array}{c}\text { Overall: } \\
60.3 \%\end{array}$ \\
\hline
\end{tabular}

Table F.7 Error matrix for the per-field single date TM classification of Landscape B.

\begin{tabular}{|r|c|c|c|c|c|c|c|c|c|}
\hline & Corn & Soy. & Hay & Pasture & Legumes & Cereals & Fallow & Aband. & $\begin{array}{c}\text { User's } \\
\text { Acc. (\%) }\end{array}$ \\
\hline Corn & 1389 & 0 & 0 & 0 & 0 & 0 & 0 & 0 & 100 \\
\hline Soybean & 167 & 1008 & 0 & 0 & 0 & 0 & 0 & 0 & 85.8 \\
\hline Hay & 0 & 0 & 223 & 117 & 39 & 0 & 15 & 0 & 56.6 \\
\hline Pasture & 0 & 0 & 50 & 0 & 0 & 0 & 0 & 0 & 0.0 \\
\hline Cegumes & 61 & 0 & 78 & 82 & 0 & 70 & 0 & 7 & 0.0 \\
\hline Fallow & 0 & 0 & 18 & 0 & 0 & 0 & 0 & 0 & 0.0 \\
\hline Abandon. & 0 & 0 & 361 & 20 & 0 & 0 & 2 & 229 & 37.4 \\
\hline $\begin{array}{r}\text { Producer's } \\
\text { Acc. (\%) }\end{array}$ & 85.9 & 100 & 28.9 & 0 & 0 & 0 & 68.5 & 97.0 & $\begin{array}{c}\text { Overall: } \\
71.9 \%\end{array}$ \\
\hline
\end{tabular}

Table F.8 Error matrix for the per-field single date TM classification of Landscape C.

\begin{tabular}{|r|c|c|c|c|c|c|c|c|c|}
\hline & Corn & Soy. & Hay & Pasture & Legumes & Cereals & Fallow & Aband. & $\begin{array}{c}\text { User's } \\
\text { Acc. (\%) }\end{array}$ \\
\hline Corn & 781 & 122 & 0 & 0 & 0 & 0 & 0 & 0 & 86.5 \\
\hline Soybean & 226 & 520 & 0 & 0 & 0 & 0 & 0 & 0 & 69.7 \\
\hline Hay & 48 & 0 & 185 & 179 & 52 & 0 & 0 & 0 & 39.9 \\
\hline Pasture & 0 & 0 & 0 & 0 & 0 & 0 & 0 & 0 & 0 \\
\hline Cegumes & 33 & 299 & 14 & 1 & 42 & 0 & 8 & 0 & 10.6 \\
\hline Fallow & 46 & 0 & 32 & 0 & 0 & 0 & 10 & 0 & - \\
\hline Abandon. & 0 & 0 & 44 & 0 & 164 & 0 & 8 & 265 & 55.1 \\
\hline $\begin{array}{r}\text { Producer's } \\
\text { Acc. (\%) }\end{array}$ & 68.9 & 55.3 & 50.5 & 0 & 16.3 & - & 45.8 & 91.1 & $\begin{array}{c}\text { Overall: } \\
55.9 \%\end{array}$ \\
\hline
\end{tabular}

Table F.9 Error matrix for the per-field single date TM classification of Landscape D.

\begin{tabular}{|r|c|c|c|c|c|c|c|c|c|}
\hline & Corn & Soy. & Hay & Pasture & Legumes & Cereals & Fallow & Aband. & $\begin{array}{c}\text { User's } \\
\text { Acc. (\%) }\end{array}$ \\
\hline Corn & 2160 & 769 & 53 & 0 & 40 & 0 & 0 & 0 & 71.5 \\
\hline Soybean & 341 & 636 & 72 & 0 & 0 & 0 & 0 & 0 & 60.6 \\
\hline Hay & 0 & 2 & 53 & 111 & 0 & 0 & 0 & 0 & 31.9 \\
\hline Pasture & 0 & 0 & 0 & 23 & 0 & 0 & 0 & 0 & 100.0 \\
\hline Legumes & 0 & 48 & 182 & 0 & 78 & 0 & 0 & 0 & 25.3 \\
\hline Cereals & 0 & 0 & 0 & 0 & 0 & 0 & 0 & 0 & - \\
\hline Fallow & 0 & 0 & 0 & 20 & 0 & 0 & 5 & 0 & 20.0 \\
\hline Abandon. & 0 & 0 & 0 & 0 & 0 & 0 & 0 & 668 & 100.0 \\
\hline $\begin{array}{r}\text { Producer's } \\
\text { Acc. (\%) }\end{array}$ & 86.4 & 43.7 & 14.7 & 14.9 & 66.1 & - & 100 & 100 & $\begin{array}{c}\text { Overall: } \\
68.9 \%\end{array}$ \\
\hline
\end{tabular}


Table F.10 Error matrix for the per-pixel multi-date TM classification of Landscape A.

\begin{tabular}{|r|c|c|c|c|c|c|c|c|c|}
\hline & Corn & Soy. & Hay & Pasture & Legumes & Cereals & Fallow & Aband. & $\begin{array}{c}\text { User's } \\
\text { Acc. (\%) }\end{array}$ \\
\hline Corn & 1301 & 43 & 2 & 0 & 0 & 2 & 1 & 0 & 96.4 \\
\hline Soybean & 167 & 832 & 1 & 4 & 0 & 0 & 5 & 0 & 82.5 \\
\hline Hay & 3 & 14 & 364 & 70 & 9 & 0 & 32 & 4 & 73.4 \\
\hline Pasture & 0 & 0 & 10 & 4 & 0 & 0 & 0 & 0 & 28.6 \\
\hline Cegumes & 16 & 43 & 216 & 3 & 110 & 0 & 2 & 0 & 28.2 \\
\hline Cereals & 15 & 9 & 3 & 1 & 0 & 90 & 3 & 0 & 74.4 \\
\hline Aballow & 4 & 3 & 6 & 1 & 0 & 0 & 14 & 14 & 33.3 \\
\hline $\begin{array}{r}\text { Producer's } \\
\text { Acc. (\%) }\end{array}$ & 0 & 0 & 10 & 2 & 0 & 0 & 17 & 17 & 37.0 \\
\hline
\end{tabular}

Table F.11 Error matrix for the per-pixel multi-date TM classification of Landscape B.

\begin{tabular}{|r|c|c|c|c|c|c|c|c|c|}
\hline & Corn & Soy. & Hay & Pasture & Legumes & Cereals & Fallow & Aband. & $\begin{array}{c}\text { User's } \\
\text { Acc. (\%) }\end{array}$ \\
\hline Corn & 1294 & 41 & 0 & 2 & 0 & 1 & 0 & 0 & 96.7 \\
\hline Soybean & 124 & 918 & 0 & 0 & 0 & 0 & 0 & 0 & 88.1 \\
\hline Hay & 160 & 46 & 571 & 166 & 16 & 13 & 44 & 15 & 55.4 \\
\hline Pasture & 0 & 0 & 11 & 2 & 0 & 0 & 8 & 0 & 9.5 \\
\hline Cegumes & 24 & 0 & 92 & 29 & 23 & 32 & 0 & 5 & 11.2 \\
\hline Fallow & 12 & 1 & 0 & 0 & 0 & 24 & 0 & 1 & 63.2 \\
\hline Abandon. & 0 & 0 & 36 & 6 & 0 & 0 & 2 & 156 & 78.0 \\
\hline $\begin{array}{r}\text { Producer's } \\
\text { Acc. (\%) }\end{array}$ & 80.0 & 91.1 & 74.0 & 0.9 & 59.0 & 34.3 & 0. & 66.1 & $\begin{array}{c}\text { Overall: } \\
74.4 \%\end{array}$ \\
\hline
\end{tabular}

Table F.12 Error matrix for the per-pixel multi-date TM classification of Landscape C.

\begin{tabular}{|r|c|c|c|c|c|c|c|c|c|}
\hline & Corn & Soy. & Hay & Pasture & Legumes & Cereals & Fallow & Aband. & $\begin{array}{c}\text { User's } \\
\text { Acc. (\%) }\end{array}$ \\
\hline Corn & 881 & 55 & 0 & 3 & 0 & 0 & 0 & 1 & 93.7 \\
\hline Soybean & 168 & 784 & 6 & 2 & 0 & 0 & 0 & 0 & 81.7 \\
\hline Hay & 56 & 79 & 259 & 166 & 104 & 0 & 4 & 17 & 37.8 \\
\hline Pasture & 0 & 0 & 0 & 0 & 16 & 0 & 0 & 0 & 0 \\
\hline Cegumes & 6 & 6 & 49 & 3 & 121 & 0 & 8 & 0 & 62.7 \\
\hline Fallow & 15 & 6 & 0 & 0 & 0 & 0 & 0 & 2 & - \\
\hline Abandon. & 0 & 0 & 8 & 1 & 0 & 0 & 22 & 230 & 88.1 \\
\hline $\begin{array}{r}\text { Producer's } \\
\text { Acc. (\%) }\end{array}$ & 77.7 & 83.3 & 70.8 & 0 & 46.9 & - & 29.2 & 79.0 & $\begin{array}{c}\text { Overall: } \\
70.5 \%\end{array}$ \\
\hline
\end{tabular}

Table F.13 Error matrix for the per-pixel multi-date TM classification of Landscape D.

\begin{tabular}{|r|c|c|c|c|c|c|c|c|c|}
\hline & Corn & Soy. & Hay & Pasture & Legumes & Cereals & Fallow & Aband. & $\begin{array}{c}\text { User's } \\
\text { Acc. (\%) }\end{array}$ \\
\hline Corn & 2253 & 699 & 37 & 0 & 38 & 0 & 0 & 0 & 74.4 \\
\hline Soybean & 185 & 695 & 0 & 0 & 0 & 0 & 0 & 0 & 79.0 \\
\hline Hay & 46 & 46 & 109 & 95 & 79 & 0 & 0 & 118 & 22.1 \\
\hline Pasture & 0 & 2 & 6 & 0 & 0 & 0 & 0 & 0 & 0 \\
\hline Legumes & 0 & 6 & 192 & 38 & 0 & 0 & 0 & 0 & 0 \\
\hline Cereals & 15 & 7 & 16 & 0 & 1 & 0 & 0 & 14 & - \\
\hline Fallow & 2 & 0 & 0 & 15 & 0 & 0 & 4 & 93 & 3.5 \\
\hline Abandon. & 0 & 0 & 0 & 6 & 0 & 0 & 1 & 443 & 98.4 \\
\hline $\begin{array}{r}\text { Producer's } \\
\text { Acc. (\%) }\end{array}$ & 90.1 & 47.8 & 30.3 & 0 & 0 & - & 80.0 & 66.3 & $\begin{array}{c}\text { Overall: } \\
66.6 \%\end{array}$ \\
\hline
\end{tabular}


Table F.14 Error matrix for the per-field multi-date TM classification of Landscape A.

\begin{tabular}{|r|c|c|c|c|c|c|c|c|c|}
\hline & Corn & Soy. & Hay & Pasture & Legumes & Cereals & Fallow & Aband. & $\begin{array}{c}\text { User's } \\
\text { Acc. (\%) }\end{array}$ \\
\hline Corn & 1444 & 0 & 0 & 0 & 0 & 0 & 0 & 0 & 100 \\
\hline Soybean & 62 & 866 & 4 & 0 & 0 & 0 & 7 & 0 & 92.2 \\
\hline Hay & 0 & 0 & 363 & 79 & 0 & 0 & 34 & 0 & 76.3 \\
\hline Pasture & 0 & 0 & 0 & 0 & 0 & 0 & 0 & 0 & 0 \\
\hline Cegumes & 0 & 78 & 230 & 0 & 119 & 0 & 3 & 0 & 27.7 \\
\hline Fallow & 0 & 0 & 0 & 0 & 0 & 92 & 0 & 0 & 100 \\
\hline Abandon. & 0 & 0 & 15 & 6 & 0 & 0 & 23 & 20 & 31.8 \\
\hline $\begin{array}{r}\text { Producer's } \\
\text { Acc. (\%) }\end{array}$ & 95.9 & 91.7 & 59.3 & 0 & 100 & 100 & 9.5 & 57.1 & $\begin{array}{c}\text { Overall: } \\
84.0 \%\end{array}$ \\
\hline
\end{tabular}

Table F.15 Error matrix for the per-field multi-date TM classification of Landscape B.

\begin{tabular}{|r|c|c|c|c|c|c|c|c|c|}
\hline & Corn & Soy. & Hay & Pasture & Legumes & Cereals & Fallow & Aband. & $\begin{array}{c}\text { User's } \\
\text { Acc. (\%) }\end{array}$ \\
\hline Corn & 1385 & 0 & 0 & 0 & 0 & 0 & 0 & 0 & 100 \\
\hline Soybean & 70 & 1008 & 0 & 0 & 0 & 0 & 0 & 0 & 93.5 \\
\hline Hay & 158 & 0 & 667 & 199 & 16 & 0 & 52 & 0 & 61.1 \\
\hline Pasture & 0 & 0 & 6 & 0 & 0 & 0 & 0 & 0 & 0 \\
\hline Cegumes & 4 & 0 & 34 & 0 & 23 & 28 & 0 & 7 & 24.0 \\
\hline Fallow & 0 & 0 & 0 & 0 & 0 & 42 & 0 & 0 & 100 \\
\hline Abandon. & 0 & 0 & 65 & 20 & 0 & 0 & 0 & 22 & 0 \\
\hline $\begin{array}{r}\text { Producer's } \\
\text { Acc. (\%) }\end{array}$ & 85.7 & 100 & 86.4 & 0 & 59.0 & 60.0 & 0 & 87.7 & $\begin{array}{c}\text { Overall: } \\
83.0 \%\end{array}$ \\
\hline
\end{tabular}

Table F.16 Error matrix for the per-field multi-date TM classification of Landscape C.

\begin{tabular}{|r|c|c|c|c|c|c|c|c|c|}
\hline & Corn & Soyb. & Hay & Pasture & Legumes & Cereals & Fallow & Aband. & $\begin{array}{c}\text { User's } \\
\text { Acc. (\%) }\end{array}$ \\
\hline Corn & 1007 & 0 & 0 & 0 & 0 & 0 & 0 & 0 & 100 \\
\hline Soybean & 94 & 877 & 9 & 0 & 0 & 0 & 0 & 0 & 89.5 \\
\hline Hay & 33 & 64 & 277 & 186 & 94 & 0 & 10 & 19 & 40.6 \\
\hline Pasture & 0 & 0 & 0 & 0 & 0 & 0 & 0 & 0 & 0 \\
\hline Cegumes & 0 & 0 & 36 & 1 & 123 & 0 & 8 & 0 & 73.2 \\
\hline Fallow & 0 & 0 & 0 & 0 & 0 & 0 & 0 & 0 & - \\
\hline Abandon. & 0 & 0 & 0 & 0 & 0 & 0 & 30 & 265 & 89.8 \\
\hline $\begin{array}{r}\text { Producer's } \\
\text { Acc. (\%) }\end{array}$ & 88.8 & 93.2 & 75.7 & 0 & 47.7 & - & 0 & 91.1 & $\begin{array}{c}\text { Overall: } \\
78.6 \%\end{array}$ \\
\hline
\end{tabular}

Table F.17 Error matrix for the per-field multi-date TM classification of Landscape D.

\begin{tabular}{|r|c|c|c|c|c|c|c|c|c|}
\hline & Corn & Soy. & Hay & Pasture & Legumes & Cereals & Fallow & Aband. & $\begin{array}{c}\text { User's } \\
\text { Acc. (\%) }\end{array}$ \\
\hline Corn & 2249 & 716 & 53 & 0 & 40 & 0 & 0 & 0 & 73.5 \\
\hline Soybean & 252 & 689 & 0 & 0 & 0 & 0 & 0 & 0 & 73.2 \\
\hline Hay & 0 & 50 & 123 & 134 & 78 & 0 & 0 & 115 & 24.6 \\
\hline Pasture & 0 & 0 & 0 & 0 & 0 & 0 & 0 & 0 & 0 \\
\hline Legumes & 0 & 0 & 184 & 0 & 0 & 0 & 0 & 0 & 0 \\
\hline Cereals & 0 & 0 & 0 & 0 & 0 & 0 & 0 & 0 & - \\
\hline Fallow & 0 & 0 & 0 & 20 & 0 & 0 & 5 & 0 & 20.0 \\
\hline Abandon. & 0 & 0 & 0 & 0 & 0 & 0 & 0 & 553 & 100 \\
\hline $\begin{array}{r}\text { Producer's } \\
\text { Acc. (\%) }\end{array}$ & 89.9 & 47.4 & 34.2 & 0 & 0 & - & 100 & 82.8 & $\begin{array}{c}\text { Overall: } \\
68.8 \%\end{array}$ \\
\hline
\end{tabular}


Table F.18 Error matrix for per-pixel QB classification of Landscape A (\# pixels/1000).

\begin{tabular}{|r|c|c|c|c|c|c|c|c|c|}
\hline & Corn & Soy. & Hay & Pasture & Legumes & Cereals & Fallow & Aband. & $\begin{array}{c}\text { User's } \\
\text { Acc. (\%) }\end{array}$ \\
\hline Corn & 116.84 & 7.83 & 7.85 & 0.19 & 0.01 & 0.00 & 0.25 & 0.79 & 87.3 \\
\hline Soybean & 63.72 & 112.61 & 2.92 & 0.04 & 0.88 & 0.00 & 0.01 & 0.25 & 62.4 \\
\hline Hay & 14.49 & 4.45 & 14.45 & 0.79 & 1.00 & 0.00 & 0.58 & 0.22 & 40.1 \\
\hline Pasture & 10.93 & 11.40 & 17.37 & 1.23 & 1.71 & 0.00 & 3.65 & 1.13 & 2.6 \\
\hline Cegumes & 26.00 & 9.27 & 24.89 & 1.15 & 5.70 & 0.00 & 0.79 & 0.19 & 8.4 \\
\hline Fallow & 0.15 & 1.42 & 18.25 & 8.47 & 4.50 & 14.72 & 0.96 & 0.12 & 30.3 \\
\hline Abandon. & 1.71 & 0.68 & 2.40 & 0.24 & 0.46 & 0.00 & 1.58 & 0.94 & 19.8 \\
\hline $\begin{array}{r}\text { Producer's } \\
\text { Acc. (\%) }\end{array}$ & 49.6 & 75.7 & 14.9 & 9.2 & 31.1 & 100 & 14.4 & 34.2 & $\begin{array}{c}\text { Overall: } \\
49.4 \%\end{array}$ \\
\hline
\end{tabular}

Table F.19 Error matrix for per-pixel QB classification of Landscape B (\# pixels/1000).

\begin{tabular}{|r|c|c|c|c|c|c|c|c|c|}
\hline & Corn & Soy. & Hay & Pasture & Legumes & Cereals & Fallow & Aband. & $\begin{array}{c}\text { User's } \\
\text { Acc. (\%) }\end{array}$ \\
\hline Corn & 136.29 & 11.10 & 13.72 & 3.64 & 0.08 & 0.03 & 0.49 & 1.58 & 81.6 \\
\hline Soybean & 26.09 & 106.90 & 24.67 & 1.18 & 0.02 & 0.01 & 0.05 & 1.26 & 66.7 \\
\hline Hay & 28.74 & 9.69 & 12.93 & 10.08 & 0.18 & 2.39 & 1.06 & 1.10 & 19.5 \\
\hline Pasture & 24.44 & 12.30 & 34.95 & 3.68 & 5.95 & 2.46 & 3.90 & 4.66 & 4.0 \\
\hline Cegumes & 33.93 & 2.35 & 7.63 & 2.49 & 0.00 & 0.25 & 0.36 & 0.15 & 0 \\
\hline Fallow & 0.71 & 0.01 & 5.43 & 0.09 & 0.00 & 0.00 & 0.00 & 0.01 & 0 \\
\hline Abandon. & 1.96 & 11.29 & 13.28 & 5.17 & 0.33 & 3.04 & 0.93 & 17.10 & 1.7 \\
\hline $\begin{array}{r}\text { Producer's } \\
\text { Acc. (\%) }\end{array}$ & 53.7 & 4.19 & 7.53 & 7.48 & 0.03 & 2.78 & 1.60 & 11.73 & 31.6 \\
\hline
\end{tabular}

Table F.20 Error matrix for per-pixel QB classification of Landscape C (\# pixels/1000).

\begin{tabular}{|r|c|c|c|c|c|c|c|c|c|}
\hline & Corn & Soy. & Hay & Pasture & Legumes & Cereals & Fallow & Aband. & $\begin{array}{c}\text { User's } \\
\text { Acc. }(\%)\end{array}$ \\
\hline Corn & 101.47 & 19.62 & 16.39 & 17.34 & 10.99 & 0.00 & 0.50 & 4.61 & 59.4 \\
\hline Soybean & 12.11 & 50.81 & 9.64 & 0.67 & 19.04 & 0.00 & 0.02 & 2.34 & 53.7 \\
\hline Hay & 7.39 & 14.60 & 2.73 & 0.50 & 1.12 & 0.00 & 0.11 & 4.63 & 8.8 \\
\hline Pasture & 29.04 & 16.21 & 7.79 & 1.87 & 2.34 & 0.00 & 1.05 & 2.74 & 3.1 \\
\hline Legumes & 3.80 & 6.56 & 5.16 & 0.04 & 1.91 & 0.00 & 0.02 & 0.44 & 10.7 \\
\hline Cereals & 0.15 & 1.02 & 0.01 & 0.17 & 0.43 & 0.00 & 1.26 & 0.04 & - \\
\hline Fallow & 15.20 & 24.99 & 9.49 & 8.73 & 0.53 & 0.00 & 3.57 & 12.49 & 4.8 \\
\hline $\begin{array}{r}\text { Producer's } \\
\text { Acc. (\%) }\end{array}$ & 5.52 & 15.09 & 6.26 & 2.61 & 3.63 & 0.00 & 1.11 & 17.79 & 32.3 \\
\hline
\end{tabular}

Table F.21 Error matrix for per-pixel QB classification of Landscape D (\# pixels/1000).

\begin{tabular}{|r|c|c|c|c|c|c|c|c|c|}
\hline & Corn & Soy. & Hay & Pasture & Legumes & Cereals & Fallow & Aband. & $\begin{array}{c}\text { User's } \\
\text { Acc. (\%) }\end{array}$ \\
\hline Corn & 261.46 & 9.61 & 6.51 & 5.45 & 1.95 & 0.00 & 0.01 & 2.94 & 90.8 \\
\hline Soybean & 57.99 & 162.53 & 26.40 & 1.48 & 1.17 & 0.00 & 0.00 & 10.03 & 62.6 \\
\hline Hay & 7.40 & 4.12 & 6.50 & 5.94 & 3.81 & 0.00 & 0.04 & 4.75 & 20.0 \\
\hline Pasture & 30.48 & 5.36 & 2.69 & 4.58 & 1.73 & 0.00 & 0.11 & 3.10 & 9.5 \\
\hline Cegumes & 15.33 & 34.48 & 12.89 & 2.51 & 8.13 & 0.00 & 0.00 & 0.61 & 11.0 \\
\hline Fallow & 0.00 & 0.00 & 0.00 & 0.01 & 0.00 & 0.00 & 0.00 & 18.92 & - \\
\hline Abandon. & 11.58 & 1.59 & 0.52 & 2.25 & 0.32 & 0.00 & 0.50 & 6.85 & 3.5 \\
\hline $\begin{array}{r}\text { Producer's } \\
\text { Acc. (\%) }\end{array}$ & 67.6 & 71.6 & 11.5 & 19.0 & 44.4 & - & 54.0 & 54.4 & $\begin{array}{c}\text { Overall: } \\
61.2 \%\end{array}$ \\
\hline
\end{tabular}


Table F.22 Error matrix for the per-field QB classification of Landscape A (\# pixels/1000).

\begin{tabular}{|r|c|c|c|c|c|c|c|c|c|}
\hline & Corn & Soy. & Hay & Pasture & Legumes & Cereals & Fallow & Aband. & $\begin{array}{c}\text { User's } \\
\text { Acc. (\%) }\end{array}$ \\
\hline Corn & 128.33 & 13.01 & 6.33 & 0.00 & 0.00 & 0.00 & 0.00 & 0.00 & 86.9 \\
\hline Soybean & 90.65 & 128.24 & 0.00 & 0.00 & 0.00 & 0.00 & 0.00 & 0.00 & 58.6 \\
\hline Hay & 0.00 & 0.00 & 8.87 & 0.00 & 0.00 & 0.00 & 0.00 & 0.00 & 100 \\
\hline Pasture & 0.00 & 7.59 & 16.51 & 1.60 & 0.00 & 0.00 & 3.77 & 1.64 & 5.2 \\
\hline Legumes & 16.57 & 0.00 & 37.19 & 1.44 & 6.73 & 0.00 & 0.00 & 0.00 & 10.9 \\
\hline Cereals & 0.00 & 0.00 & 27.83 & 8.51 & 11.62 & 14.73 & 0.95 & 0.00 & 23.1 \\
\hline Fallow & 0.00 & 0.00 & 0.00 & 0.00 & 0.00 & 0.00 & 0.00 & 0.00 & 0 \\
\hline $\begin{array}{r}\text { Abandon. } \\
\text { Producer's }\end{array}$ & 0.00 & 0.00 & 0.00 & 1.73 & 0.00 & 0.00 & 6.30 & 3.89 & 32.7 \\
Acc. (\%) & 54.5 & 86.2 & 9.2 & 12.1 & 36.7 & 100 & 0 & 70.4 & $\begin{array}{c}\text { Overall: } \\
53.7 \%\end{array}$ \\
\hline
\end{tabular}

Table F.23 Error matrix for the per-field QB classification of Landscape B (\# pixels/1000).

\begin{tabular}{|r|c|c|c|c|c|c|c|c|c|}
\hline & Corn & Soy. & Hay & Pasture & Legumes & Cereals & Fallow & Aband. & $\begin{array}{c}\text { User's } \\
\text { Acc. (\%) }\end{array}$ \\
\hline Corn & 212.94 & 0.04 & 0.00 & 0.00 & 0.00 & 0.00 & 0.00 & 0.00 & 100 \\
\hline Soybean & 0.00 & 148.48 & 30.00 & 0.00 & 0.00 & 0.00 & 0.00 & 0.00 & 83.2 \\
\hline Hay & 16.55 & 0.00 & 25.37 & 11.07 & 0.00 & 0.00 & 0.00 & 0.00 & 47.9 \\
\hline Legumes & 0.00 & 0.00 & 39.15 & 3.08 & 6.58 & 4.42 & 5.84 & 0.92 & 5.1 \\
\hline Cereals & 0.68 & 0.00 & 12.23 & 0.00 & 0.00 & 0.00 & 0.00 & 0.00 & 0 \\
\hline Fallow & 0.00 & 5.86 & 8.08 & 3.04 & 0.00 & 0.00 & 0.32 & 11.98 & 1.1 \\
\hline Abandon. & 0.00 & 3.44 & 5.29 & 16.63 & 0.00 & 6.54 & 2.24 & 24.67 & 41.9 \\
\hline $\begin{array}{r}\text { Producer's } \\
\text { Acc. (\%) }\end{array}$ & 83.9 & 94.1 & 21.1 & 9.1 & 0 & 0 & 3.9 & 65.7 & $\begin{array}{c}\text { Overall: } \\
65.9 \%\end{array}$ \\
\hline
\end{tabular}

Table F.24 Error matrix for the per-field QB classification of Landscape C (\# pixels/1000).

\begin{tabular}{|r|c|c|c|c|c|c|c|c|c|}
\hline & Corn & Soy. & Hay & Pasture & Legumes & Cereals & Fallow & Aband. & $\begin{array}{c}\text { User's } \\
\text { Acc. }(\%)\end{array}$ \\
\hline Corn & 121.65 & 17.39 & 28.62 & 28.78 & 9.59 & 0.00 & 0.00 & 1.33 & 58.7 \\
\hline Soybean & 0.00 & 59.56 & 5.77 & 0.00 & 25.54 & 0.00 & 0.00 & 0.00 & 65.5 \\
\hline Hay & 0.00 & 18.86 & 0.00 & 0.00 & 0.00 & 0.00 & 0.00 & 0.00 & 0 \\
\hline Pasture & 46.18 & 0.00 & 3.51 & 0.00 & 0.00 & 0.00 & 1.39 & 0.00 & 0 \\
\hline Cegumes & 0.00 & 0.00 & 5.55 & 0.00 & 0.00 & 0.00 & 0.00 & 0.00 & 0 \\
\hline Fallow & 0.00 & 0.00 & 0.00 & 0.14 & 0.00 & 0.00 & 1.29 & 0.00 & - \\
\hline Abandon. & 9.84 & 29.28 & 14.01 & 1.57 & 0.00 & 0.00 & 4.97 & 0.91 & 9.8 \\
\hline $\begin{array}{r}\text { Producer's } \\
\text { Acc. (\%) }\end{array}$ & 68.5 & 40.0 & 0 & 0 & 0 & - & 65.0 & 95.0 & $\begin{array}{c}\text { Overall: } \\
45.0 \%\end{array}$ \\
\hline
\end{tabular}

Table F.25 Error matrix for the per-field QB classification of Landscape D (\# pixels/1000).

\begin{tabular}{|r|c|c|c|c|c|c|c|c|c|}
\hline & Corn & Soy. & Hay & Pasture & Legumes & Cereals & Fallow & Aband. & $\begin{array}{c}\text { User's } \\
\text { Acc. (\%) }\end{array}$ \\
\hline Corn & 351.64 & 6.42 & 8.12 & 0.00 & 6.06 & 0.00 & 0.00 & 0.00 & 94.5 \\
\hline Soybean & 19.09 & 186.48 & 20.73 & 0.00 & 0.00 & 0.00 & 0.00 & 0.00 & 82.4 \\
\hline Hay & 0.00 & 0.11 & 1.19 & 12.00 & 0.00 & 0.00 & 0.00 & 0.00 & 8.9 \\
\hline Pasture & 15.80 & 1.49 & 7.25 & 5.39 & 0.00 & 0.00 & 0.00 & 0.00 & 18.0 \\
\hline Legumes & 0.00 & 27.07 & 19.26 & 3.70 & 12.24 & 0.00 & 0.00 & 0.00 & 19.7 \\
\hline Cereals & 0.00 & 0.00 & 0.00 & 0.00 & 0.00 & 0.00 & 0.00 & 17.88 & - \\
\hline Fallow & 0.00 & 0.00 & 0.00 & 3.04 & 0.00 & 0.00 & 0.92 & 0.00 & 23.2 \\
\hline $\begin{array}{r}\text { Abandon. } \\
\text { Producer's }\end{array}$ & 0.00 & 5.51 & 0.00 & 0.00 & 0.00 & 0.00 & 0.00 & 85.68 & 94.0 \\
Acc. (\%) & 91.0 & 82.1 & 2.1 & 22.3 & 66.9 & - & 100 & 82.7 & $\begin{array}{c}\text { Overall: } \\
78.8 \%\end{array}$ \\
\hline
\end{tabular}


Table F.26 Error matrix for the per-field multi-date TM classification of Landscape A, with merged classes.

\begin{tabular}{|r|c|c|c|c|c|c|}
\hline & Corn & Soybean & Hay/Past./Legum. & Cereals & Fallow/Aband. & $\begin{array}{c}\text { User's } \\
\text { Acc. (\%) }\end{array}$ \\
\hline Corn & 1409 & 0 & 0 & 0 & 7 & 99.5 \\
\hline Soybean & 62 & 866 & 0 & 0 & 7 & 92.6 \\
\hline Hay/Past./Legum. & 35 & 78 & 795 & 0 & 37 & 84.1 \\
\hline Cereals & 0 & 0 & 0 & 92 & 0 & 100 \\
\hline Fallow/Aband. & 0 & 0 & 21 & 0 & 58 & 73.4 \\
\hline $\begin{array}{r}\text { Producer's } \\
\text { Acc. (\%) }\end{array}$ & 93.6 & 91.7 & 97.4 & 100 & 53.2 & $\begin{array}{c}\text { Overall: } \\
92.9 \%\end{array}$ \\
\hline
\end{tabular}

Table F.27 Error matrix for the per-field multi-date TM classification of Landscape B, with merged classes.

\begin{tabular}{|r|c|c|c|c|c|c|}
\hline & Corn & Soybean & Hay/Past./Legum. & Cereals & Fallow/Aband. & $\begin{array}{c}\text { User's } \\
\text { Acc. (\%) }\end{array}$ \\
\hline Corn & 1385 & 0 & 0 & 0 & 0 & 100 \\
\hline Soybean & 70 & 1008 & 0 & 0 & 0 & 93.5 \\
\hline Hay/Past./Legum. & 162 & 0 & 819 & 28 & 59 & 76.7 \\
\hline Cereals & 0 & 0 & 0 & 42 & 0 & 100 \\
\hline Fallow/Aband. & 0 & 0 & 211 & 0 & 231 & 52.3 \\
\hline $\begin{array}{r}\text { Producer's } \\
\text { Acc. (\%) }\end{array}$ & 85.7 & 100 & 79.5 & 60.0 & 79.7 & $\begin{array}{c}\text { Overall: } \\
86.8 \%\end{array}$ \\
\hline
\end{tabular}

Table F.28 Error matrix for the per-field multi-date TM classification of Landscape C, with merged classes.

\begin{tabular}{|r|c|c|c|c|c|c|}
\hline & Corn & Soybean & Hay/Past./Legum. & Cereals & Fallow/Aband. & $\begin{array}{c}\text { User's } \\
\text { Acc. (\%) }\end{array}$ \\
\hline Corn & 951 & 0 & 0 & 0 & 0 & 100 \\
\hline Soybean & 137 & 877 & 0 & 0 & 0 & 86.5 \\
\hline Hay/Past./Legum. & 0 & 64 & 760 & 0 & 8 & 91.3 \\
\hline Cereals & 0 & 0 & 0 & 0 & 0 & - \\
\hline Fallow/Aband. & 46 & 0 & 71 & 0 & 331 & 73.9 \\
\hline $\begin{array}{r}\text { Producer's } \\
\text { Acc. (\%) }\end{array}$ & 83.9 & 93.2 & 91.5 & - & 97.6 & $\begin{array}{c}\text { Overall: } \\
90.0 \%\end{array}$ \\
\hline
\end{tabular}

Table F.29 Error matrix for the per-field multi-date TM classification of Landscape D, with merged classes.

\begin{tabular}{|r|c|c|c|c|c|c|}
\hline & Corn & Soybean & Hay/Past./Legum. & Cereals & Fallow/Aband. & $\begin{array}{c}\text { User's } \\
\text { Acc. (\%) }\end{array}$ \\
\hline Corn & 2415 & 495 & 93 & 0 & 0 & 80.4 \\
\hline Soybean & 56 & 910 & 0 & 0 & 0 & 94.2 \\
\hline Hay/Past./Legum. & 30 & 50 & 519 & 0 & 0 & 86.6 \\
\hline Cereals & 0 & 0 & 0 & 0 & 0 & - \\
\hline Fallow/Aband. & 0 & 0 & 20 & 0 & 673 & 97.1 \\
\hline $\begin{array}{r}\text { Producer's } \\
\text { Acc. (\%) }\end{array}$ & 96.6 & 62.5 & 82.1 & - & 100 & $\begin{array}{c}\text { Overall: } \\
85.9 \%\end{array}$ \\
\hline
\end{tabular}




\section{Appendix G: Examples of poorly classified fields with uncertain ground referencing [study area classification]}

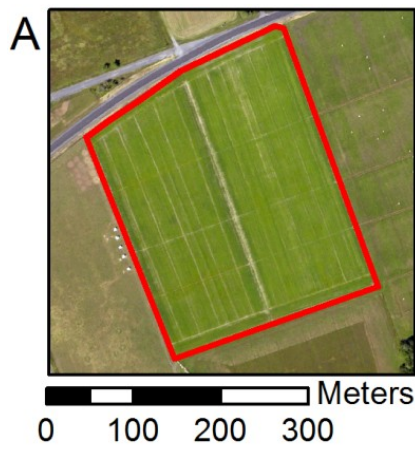

Largest case for Hay.

Ground reference team noted, "ploughed. cereal?" suggesting that the crop type was not very clear and may have been Cereals.

Field appeared to be atypical of the nearby Hay fields.

1: Soybean $(60.8 \%)$

2: Corn $(34.1 \%)$

3: Cereals $(3.8 \%)$

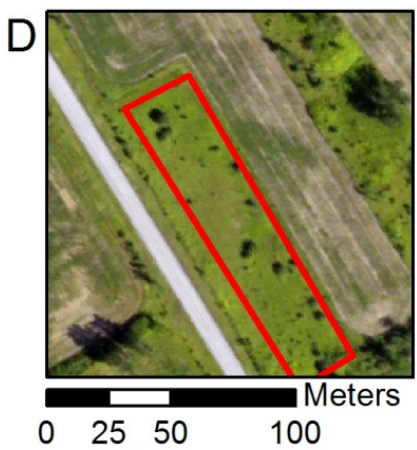

Case for Soybean.

Strongly appears to be Abandoned (not a field) in air photo. The field to the immediate North and East is Soybean.

1: Fallow $(51.1 \%)$

2: Pasture $(29.3 \%)$

3: Hay $(13.9 \%)$

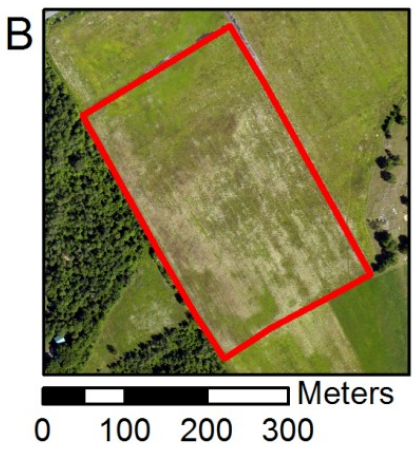

Second largest case for Hay. No ground reference team comment, but crop appears to have severe health issues in air photo, with a fair amount of exposed soil.

1: Cereals $(32.9 \%)$

2: Fallow $(18.8 \%)$

3: Pasture (16.4\%)
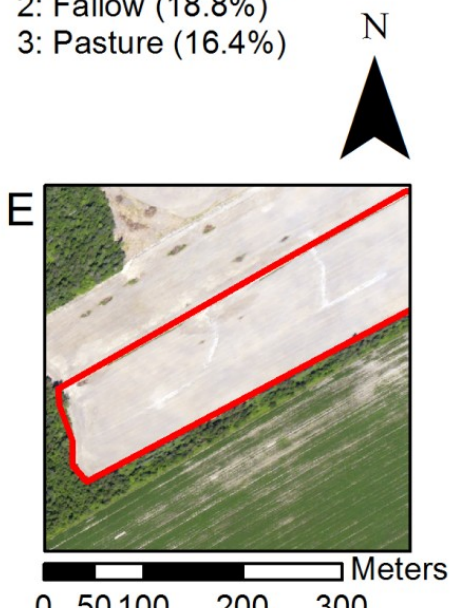

Case for Fallow. Ground reference team noted, "or hay" suggesting that it may have been Hay.

1: Hay $(37.1 \%)$

2: Cereals $(19.8 \%)$

3: Soybean $(17.9 \%)$

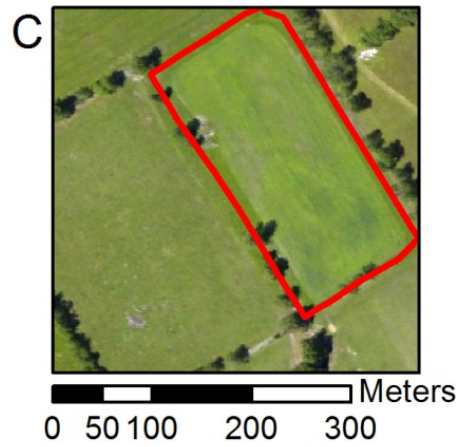

Case for Pasture. Ground reference team noted, "planted" suggesting that it had been referenced early in the growing season. In the air photo, vegetation appears to have been cut rather than grazed. Field to the Southwest is Pasture. 1: Hay $(23.0 \%)$

2: Soybean (19.4\%)

3: Legumes $(18.0 \%)$

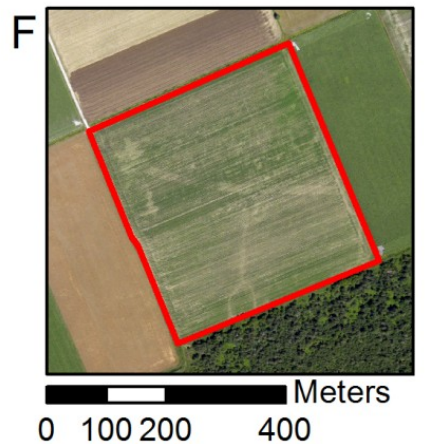

Largest case for Legumes. Ground reference team noted, "lots of dandelions."

1: Fallow $(42.5 \%)$

2: Hay $(37.4 \%)$

3: Pasture (13.9\%)

Figure G.1 Cases where (1) most-likely, (2) second most-likely, and (3) third mostlikely class did not match the ground referenced class. Cases are shown for (A, B) Hay, (C) Pasture, (D) Soybean, (E) Fallow, and (F) Legumes. Probabilities of class membership are included for the top three classes, as obtained from the Random Forest classifier. 
Appendix H: Hard and simulated biodiversity model coefficients for all diversity types (including abundance)
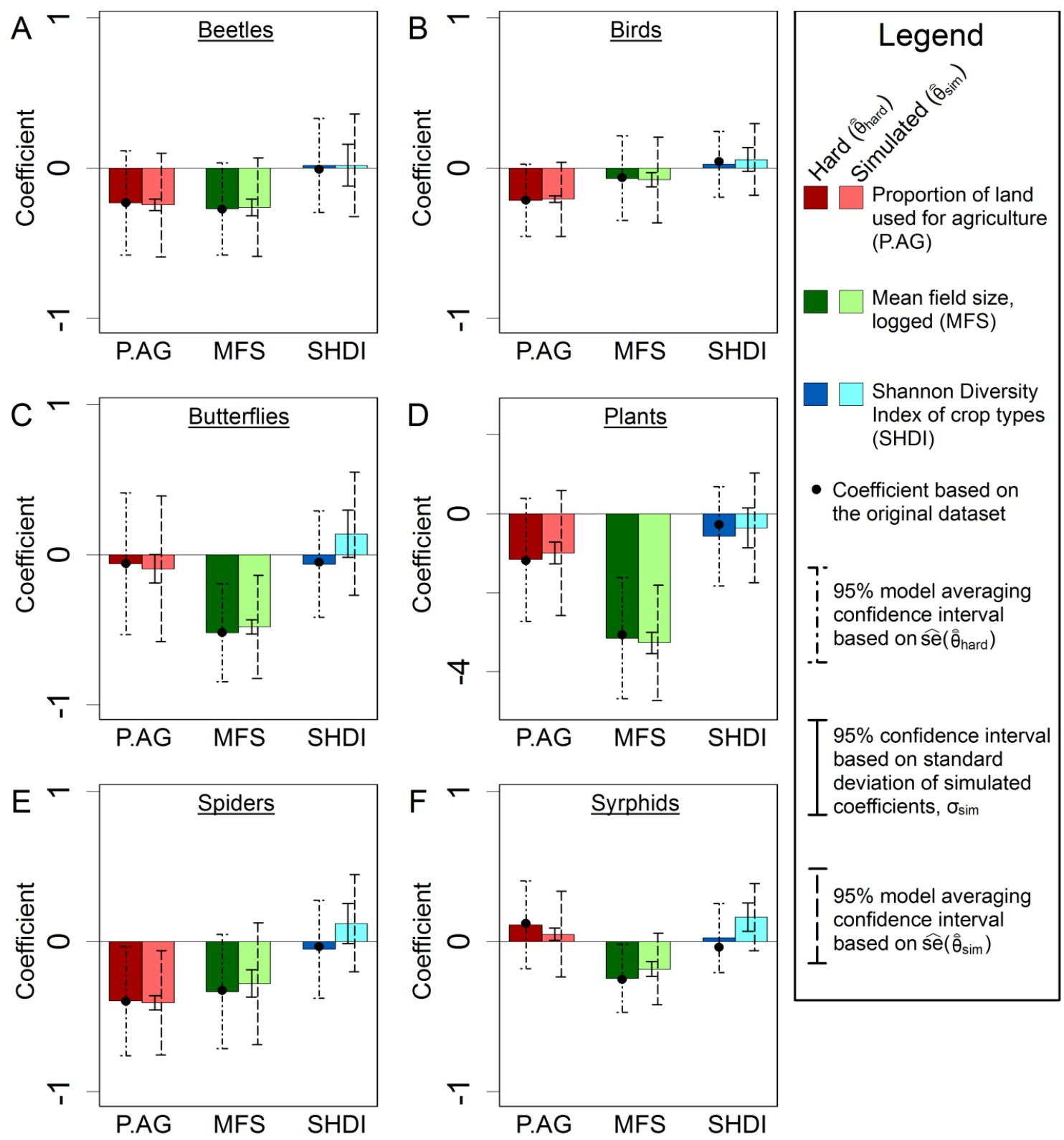

ce interval deviation of simulated

T based on $\widehat{\operatorname{se}}\left(\hat{\theta}_{\text {sim }}\right)$

Figure H.1 (alpha) Hard and simulated (independent fields approach) regression coefficients and confidence intervals for the $(\mathbf{A})$ beetles, $(\mathbf{B})$ birds, (C) butterflies, (D) plants, (E) spiders, and (F) syrphids alpha diversity models. 

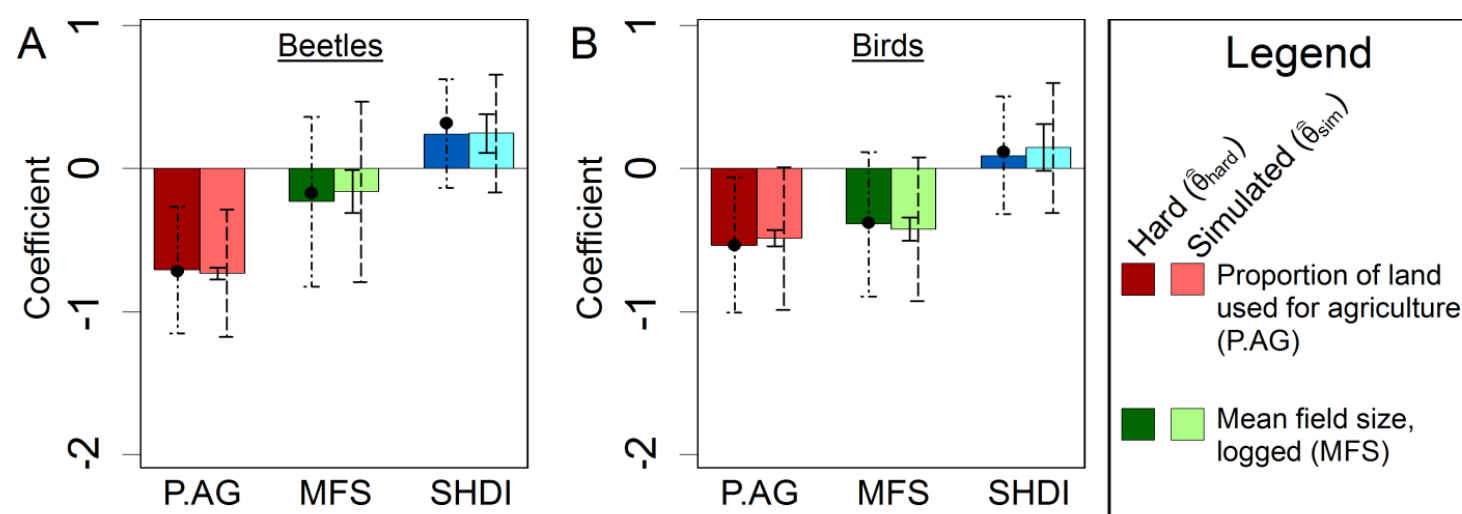
used for agriculture (P.AG)
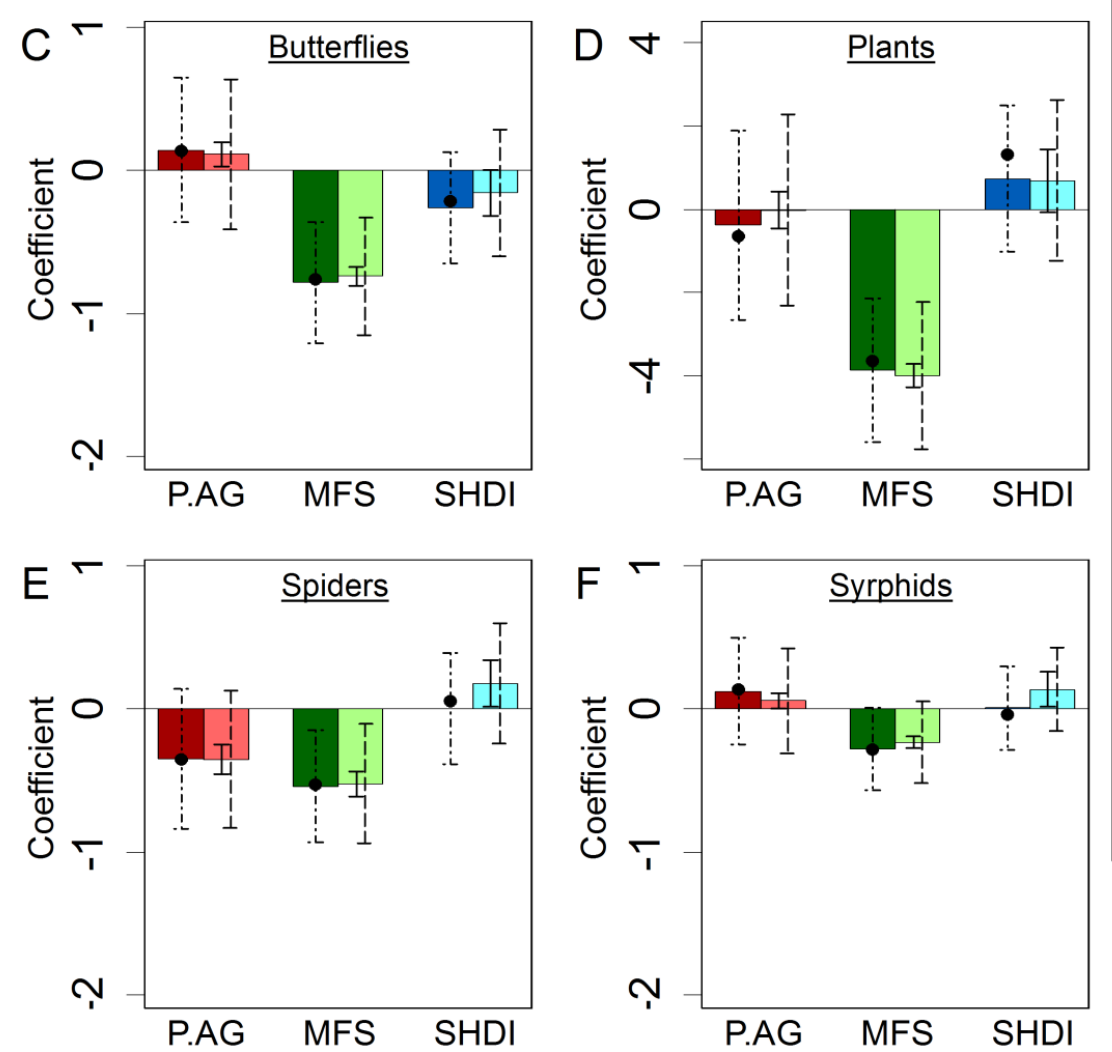

$95 \%$ confidence interval based on standard deviation of simulated $\perp$ coefficients, $\sigma_{\text {sim }}$

T $95 \%$ model averaging confidence interval based on $\widehat{\operatorname{se}}\left(\hat{\theta}_{\mathrm{sim}}\right)$

Figure H.2 (beta) Hard and simulated (independent fields approach) regression coefficients and confidence intervals for the $(\mathbf{A})$ beetles, $(\mathbf{B})$ birds, (C) butterflies, (D) plants, (E) spiders, and (F) syrphids beta diversity models. 

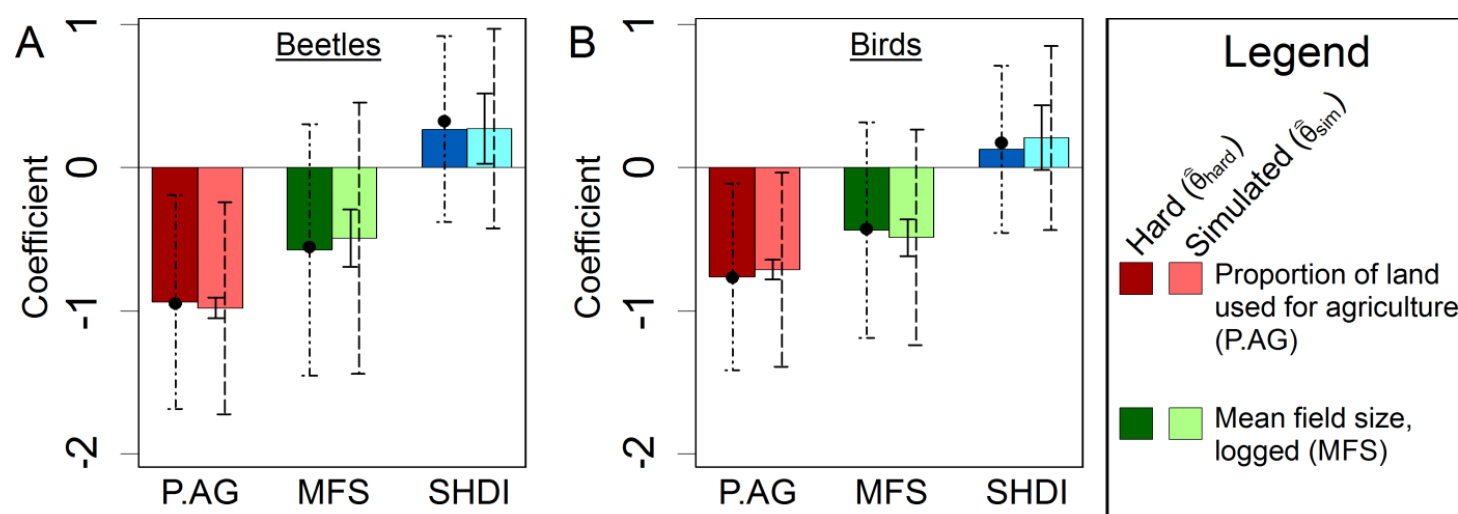
used for agriculture (P.AG)
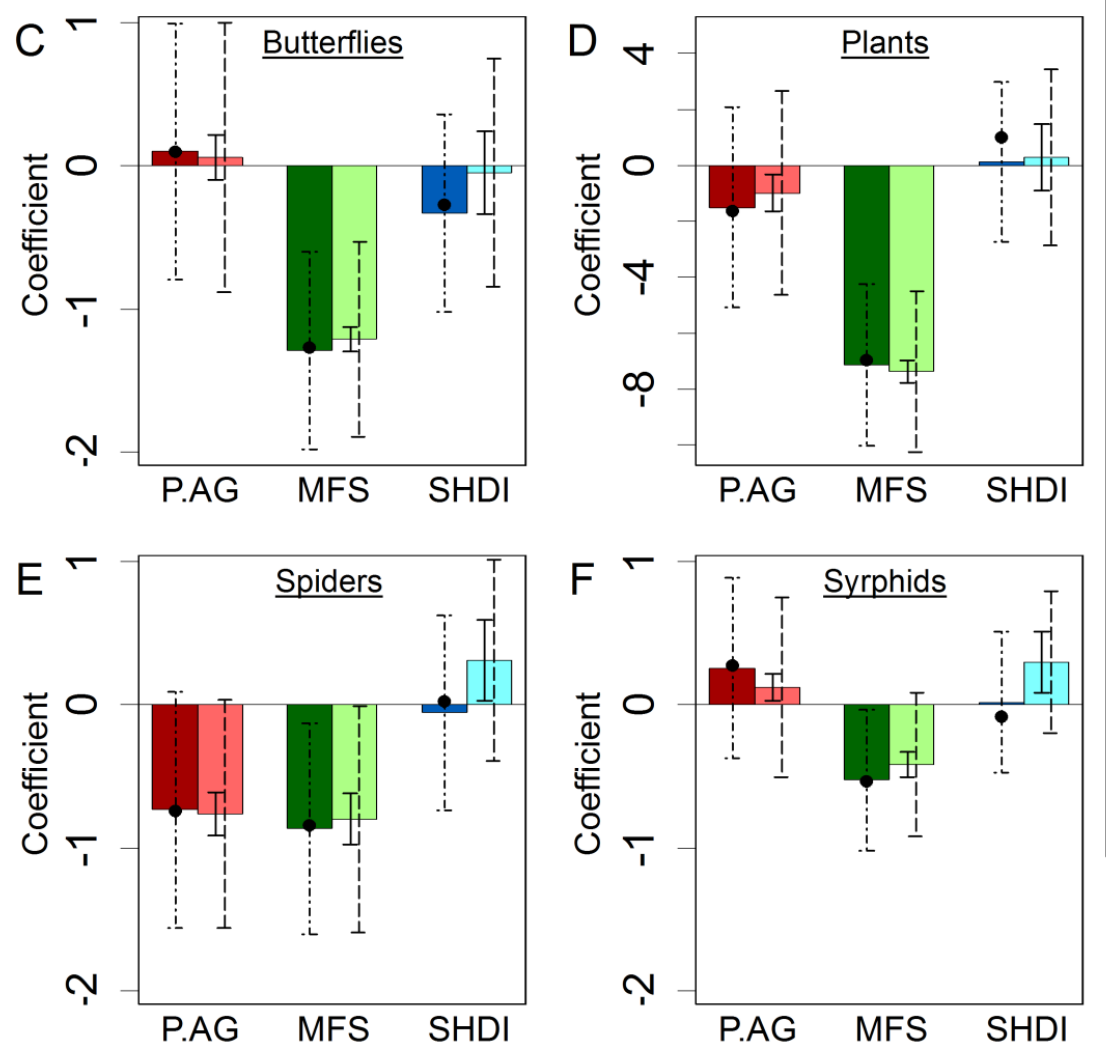

$95 \%$ confidence interval based on standard deviation of simulated $\perp$ coefficients, $\sigma_{\text {sim }}$

T $95 \%$ model averaging confidence interval based on $\widehat{\operatorname{se}}\left(\hat{\theta}_{\text {sim }}\right)$

Figure H.3 (gamma) Hard and simulated (independent fields approach) regression coefficients and confidence intervals for the $(\mathbf{A})$ beetles, $(\mathbf{B})$ birds, (C) butterflies, (D) plants, (E) spiders, and (F) syrphids gamma diversity models. 

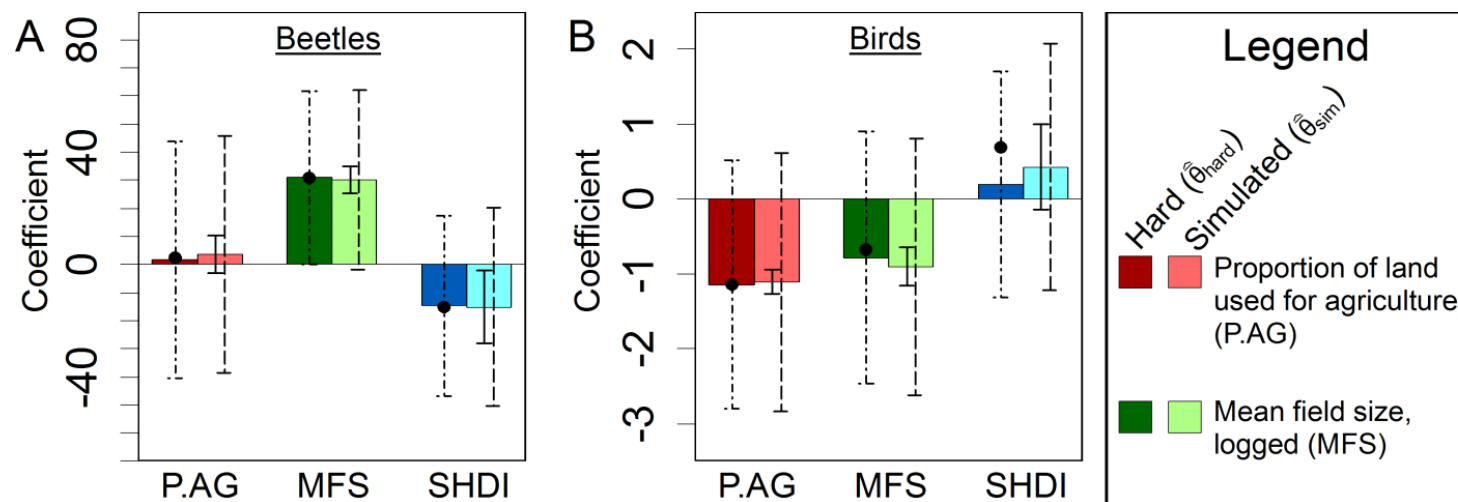
used for agriculture (P.AG)

Mean field size logged (MFS)
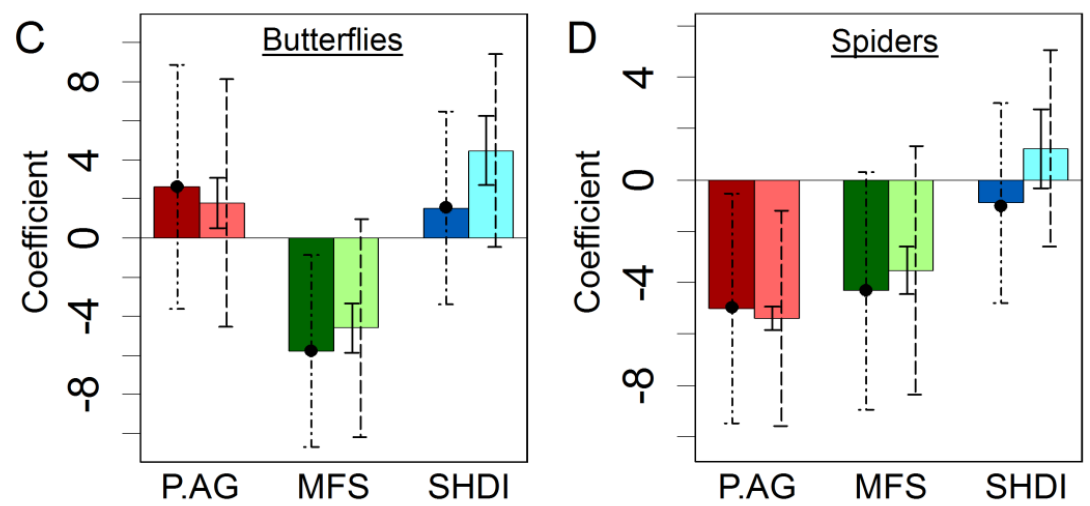

Shannon Diversity Index of crop types (SHDI)

- Coefficient based on the original dataset

7

i 95\% model averaging i confidence interval i based on $\widehat{\operatorname{se}}\left(\hat{\theta}_{\text {hard }}\right)$

-

$95 \%$ confidence interval based on standard deviation of simulated

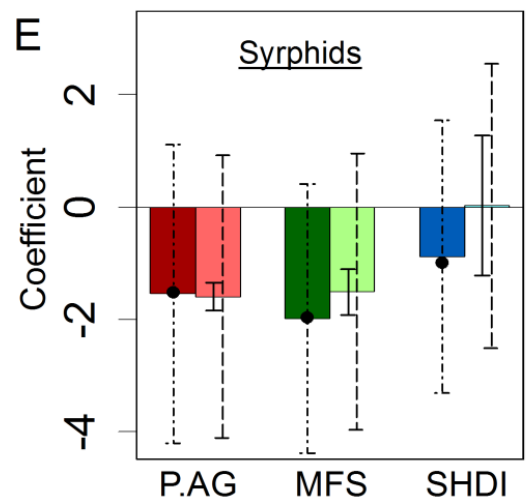
coefficients, $\sigma_{\text {sim }}$

$T$

95\% model averaging confidence interval based on $\widehat{\operatorname{se}}\left(\hat{\theta}_{\text {sim }}\right)$

Figure H.4 (abundance) Hard and simulated (independent fields approach) regression coefficients and confidence intervals for the $(\mathbf{A})$ beetles, $(\mathbf{B})$ birds, (C) butterflies, (D) plants, (E) spiders, and (F) syrphids abundance models. There were no abundance metrics for plants. 


\section{Appendix I: Uncertainty variance ratios for all models and simulation approaches}

Table I.1 The proportion of $\sigma_{\text {sim }}^{2}$ to $\widehat{\operatorname{var}}\left(\hat{\bar{\theta}}_{\text {sim }}\right)$, by model, for the model averaged coefficient estimates. Proportions are shown by simulation approach, including the independent field (Ind.Field), locally-grouped (Loc.Gr.), and landscape-grouped (Land.Gr.) approaches. See Section 4.2.4.3.

\begin{tabular}{|c|c|c|c|c|c|c|c|c|c|c|}
\hline \multirow[b]{2}{*}{ Taxon } & \multirow{2}{*}{$\begin{array}{c}\text { Diversity } \\
\text { Type }\end{array}$} & \multicolumn{3}{|c|}{ P.AG Coefficient } & \multicolumn{3}{|c|}{ MFS Coefficient } & \multicolumn{3}{|c|}{ SHDI Coefficient } \\
\hline & & Ind.Field & Loc.Gr. & Land.Gr. & Ind.Field & Loc.Gr. & Land.Gr. & Ind.Field & Loc.Gr. & Land.Gr. \\
\hline Beetles & Alpha & 0.0123 & 0.0119 & 0.0129 & 0.0286 & 0.0305 & 0.0360 & 0.1665 & 0.2227 & 0.2807 \\
\hline Beetles & Beta & 0.0088 & 0.0081 & 0.0080 & 0.0581 & 0.0603 & 0.0611 & 0.1094 & 0.1307 & 0.1619 \\
\hline Beetles & Gamma & 0.0096 & 0.0091 & 0.0089 & 0.0451 & 0.0464 & 0.0473 & 0.1234 & 0.1587 & 0.1981 \\
\hline Beetles & Abund. & 0.0262 & 0.0267 & 0.0278 & 0.0221 & 0.0221 & 0.0256 & 0.1366 & 0.1476 & 0.1846 \\
\hline Birds & Alpha & 0.0075 & 0.0079 & 0.0077 & 0.0273 & 0.0304 & 0.0323 & 0.1127 & 0.1618 & 0.2229 \\
\hline Birds & Beta & 0.0133 & 0.0140 & 0.0142 & 0.0269 & 0.0305 & 0.0325 & 0.1284 & 0.1801 & 0.2622 \\
\hline Birds & Gamma & 0.0099 & 0.0104 & 0.0103 & 0.0288 & 0.0324 & 0.0337 & 0.1209 & 0.1708 & .2442 \\
\hline Birds & Abund. & 085 & 0.0087 & 0.0083 & 0.0222 & 0.0255 & 0.0270 & 1201 & 0.1699 & 2331 \\
\hline Butterflies & Alpha & 0.0370 & 0.0385 & 0.0393 & 0.0188 & 0.0203 & 0.0209 & 0.1425 & 0.2086 & 0.2834 \\
\hline Butterflies & Beta & 0.0257 & 0.0305 & 0.0329 & 0.0262 & 0.0353 & 0.0441 & 0.1267 & 0.1762 & 0.2674 \\
\hline Butterflies & Gam & 74 & 1 & 2 & 63 & 0.0212 & 8 & 08 & 36 & 2709 \\
\hline Butterflies & Abund. & 0.0404 & 0.0469 & 0.0498 & 0.0516 & 0.0618 & 0.0665 & 0.1257 & 0.2324 & 0.3588 \\
\hline Plants & Alpha & 0.0318 & 0.0359 & 352 & 0342 & 0.0475 & .0479 & 0.1349 & 0.1705 & 0.2000 \\
\hline Plants & Beta & 0.0363 & 0.0380 & 0.0390 & 0.0266 & 0.0269 & 0.0305 & 0.1526 & 0.1727 & 0.2234 \\
\hline Plants & Gamma & 0.0320 & 0.0337 & 0.0335 & 0.0198 & 0.0216 & 0.0221 & 0.1423 & 0.1625 & 0.2034 \\
\hline Spiders & Alpha & 178 & 0.0199 & 208 & 498 & 0.0566 & 0.0588 & 1689 & 0.2906 & 3789 \\
\hline Spiders & Beta & 0.0442 & 0.0477 & 0.0465 & 0.0442 & 0.0443 & 0.0423 & 0.1563 & 0.2140 & 0.2801 \\
\hline Spiders & Gamma & 0.0354 & 0.0400 & 0.0403 & 0.0514 & 0.0534 & 0.0514 & 0.1628 & 0.2578 & 0.3346 \\
\hline Spiders & Abund. & 0.0123 & 0.0147 & 0.0159 & 0.0352 & 0.0458 & 0.0499 & 0.1593 & 0.3074 & 0.3915 \\
\hline Syrphids & Alpha & 0.0210 & 0.0258 & 0.0286 & 0.0420 & 0.0477 & 0.0413 & 0.1752 & 0.3112 & 0.3836 \\
\hline Syrphids & Beta & 0.0204 & 0.0221 & 0.0251 & 0.0223 & 0.0253 & 0.0276 & 0.1812 & 0.2607 & 0.3301 \\
\hline Syrphids & Gamma & 0.0225 & 0.0255 & 0.0287 & 0.0301 & 0.0340 & 0.0327 & 0.1830 & 0.2873 & 0.3552 \\
\hline Syrphids & Abund. & 0.0101 & 0.0111 & 0.0128 & 0.0274 & 0.0383 & 0.0506 & 0.2437 & 0.3241 & 0.3991 \\
\hline
\end{tabular}


Appendix J: Convergence plots across different simulation approaches
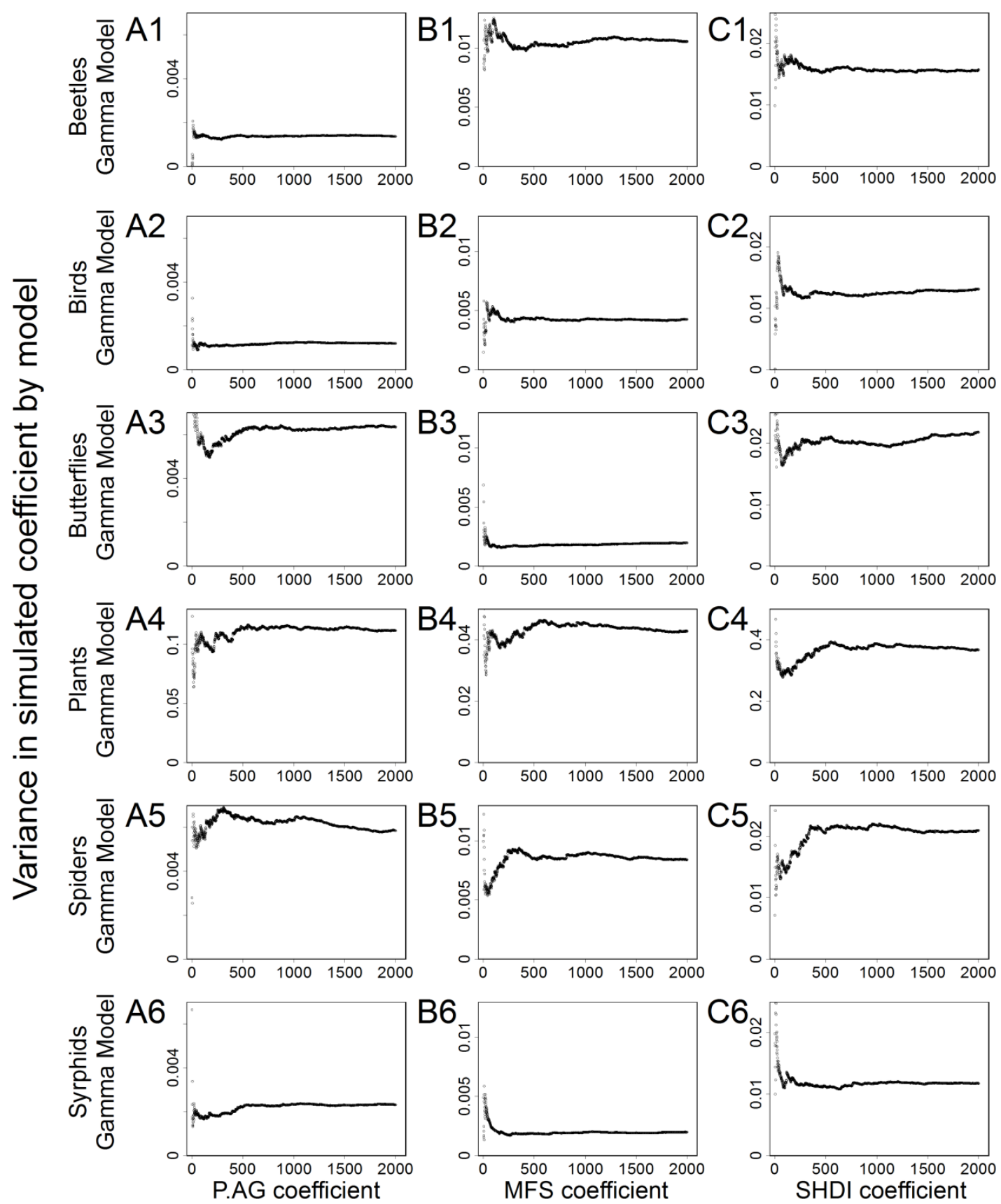

Number of realizations by coefficient

Figure J.1 (independent field) Cumulative variance in simulated coefficient

versus the number of realizations used to calculate this variance. The values shown are for the gamma diversity models and are based on the independent field simulation approach. 

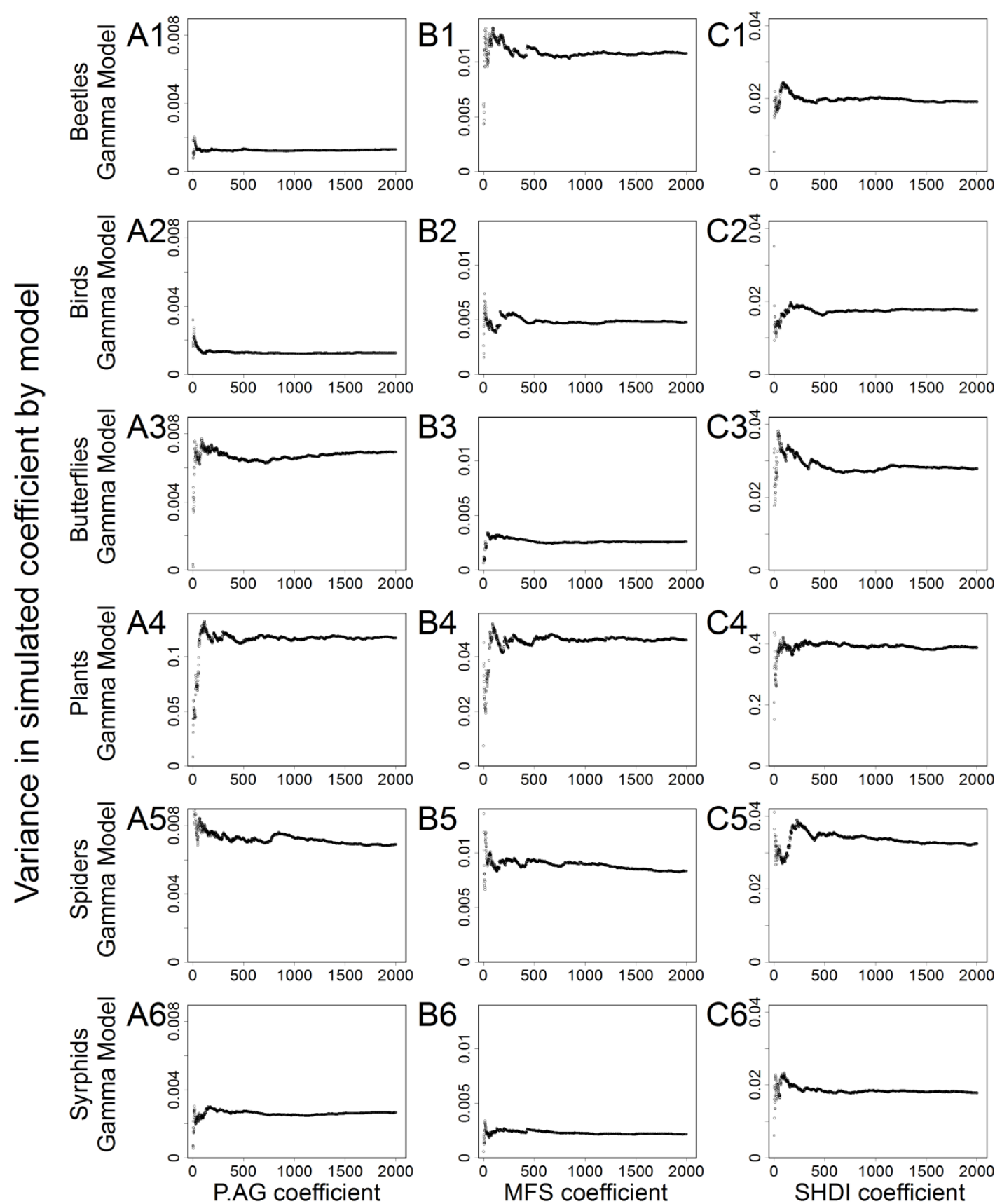

Number of realizations by coefficient

Figure J.2 (locally-grouped) Cumulative variance in simulated coefficient versus the number of realizations used to calculate this variance. The values shown are for the gamma diversity models and are based on the locallygrouped simulation approach. 

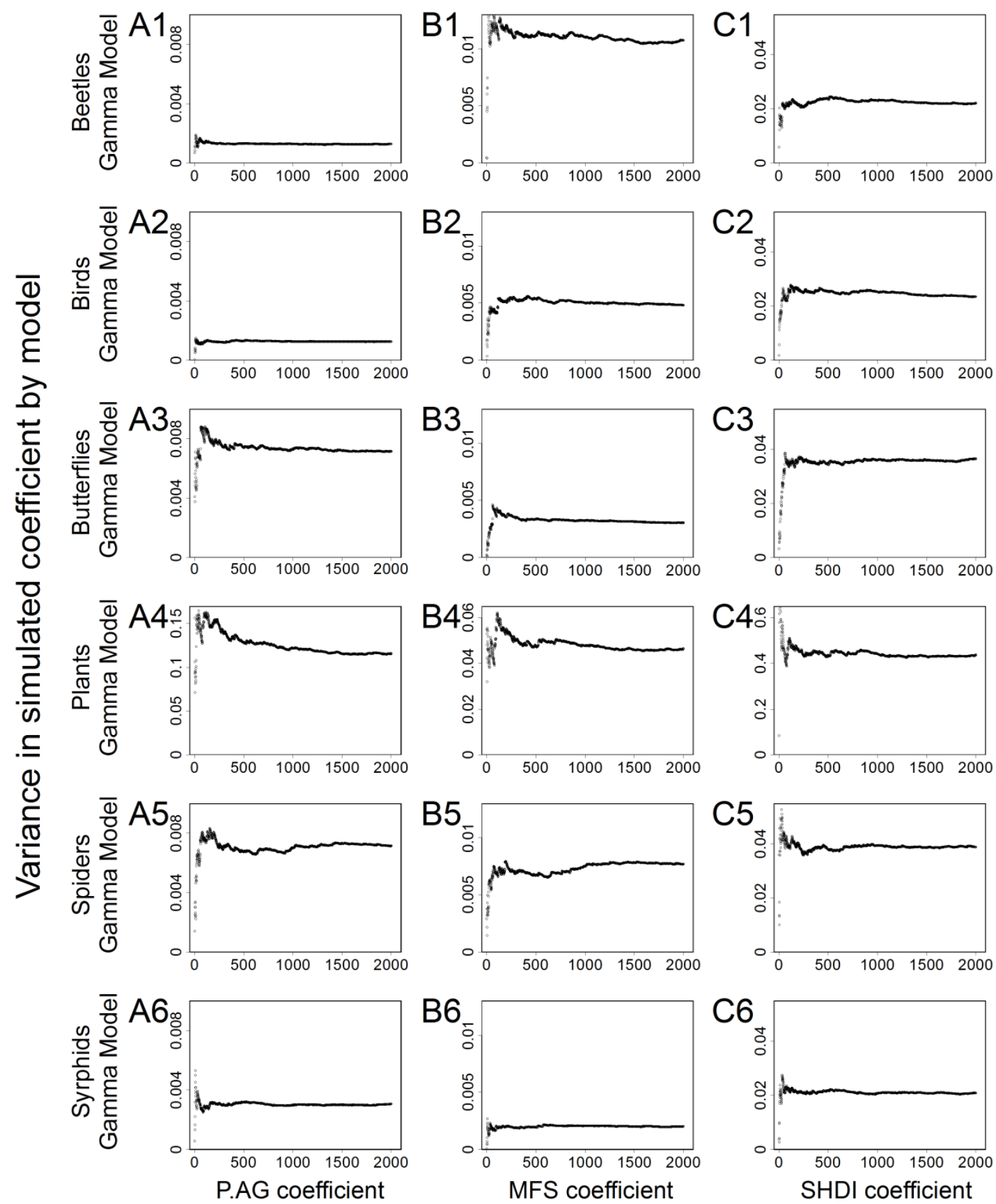

Number of realizations by coefficient

Figure J.3 (landscape-grouped) Cumulative variance in simulated coefficient versus the number of realizations used to calculate this variance. The values shown are for the gamma diversity models and are based on the landscapegrouped simulation approach. 


\section{Appendix K: Simulated landscape metrics for all LCs.}

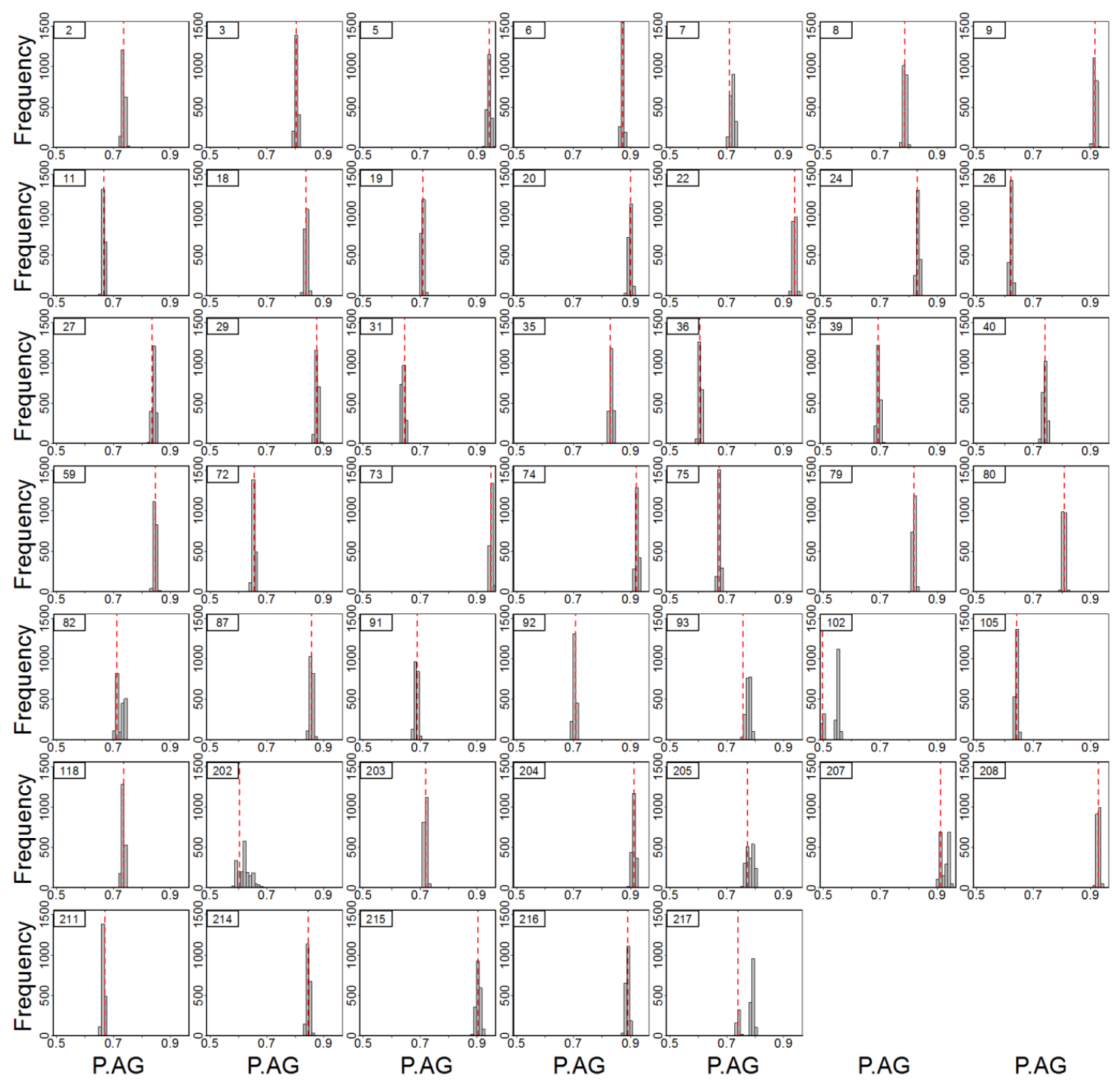

Figure K.1 P.AG metric distributions for all year-2012 LCs. The distributions are labeled in the upper left corner, with numbers corresponding to the Farmland Biodiversity Project landscape numbering system. 

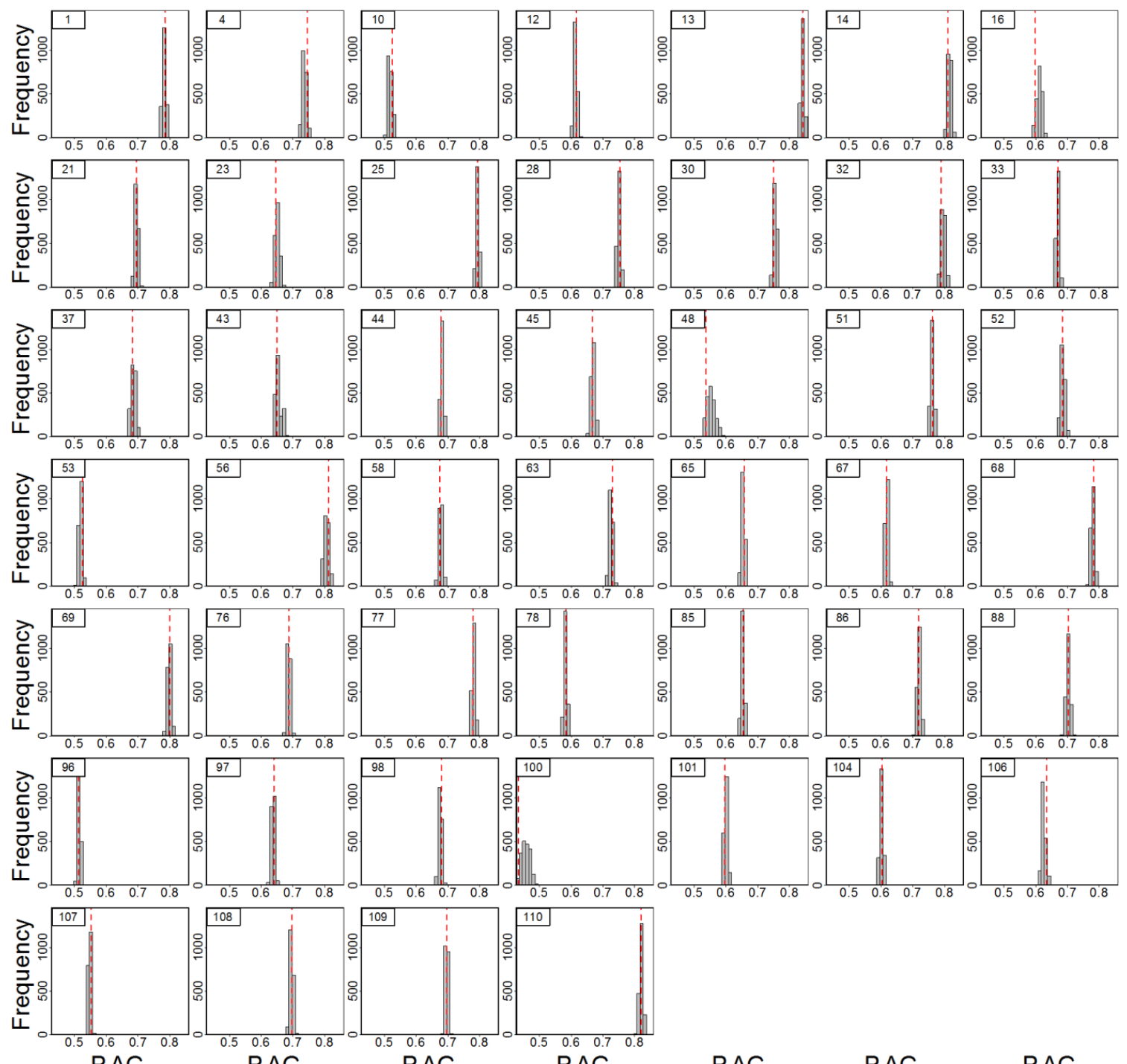

$\begin{array}{lll}\text { P.AG P.AG P.AG } & \end{array}$

Figure J.2 P.AG metric distributions for all year-2011 LCs. The distributions are labeled in the upper left corner, with numbers corresponding to the Farmland Biodiversity Project landscape numbering system. The dashed red line represents the hard metric. 

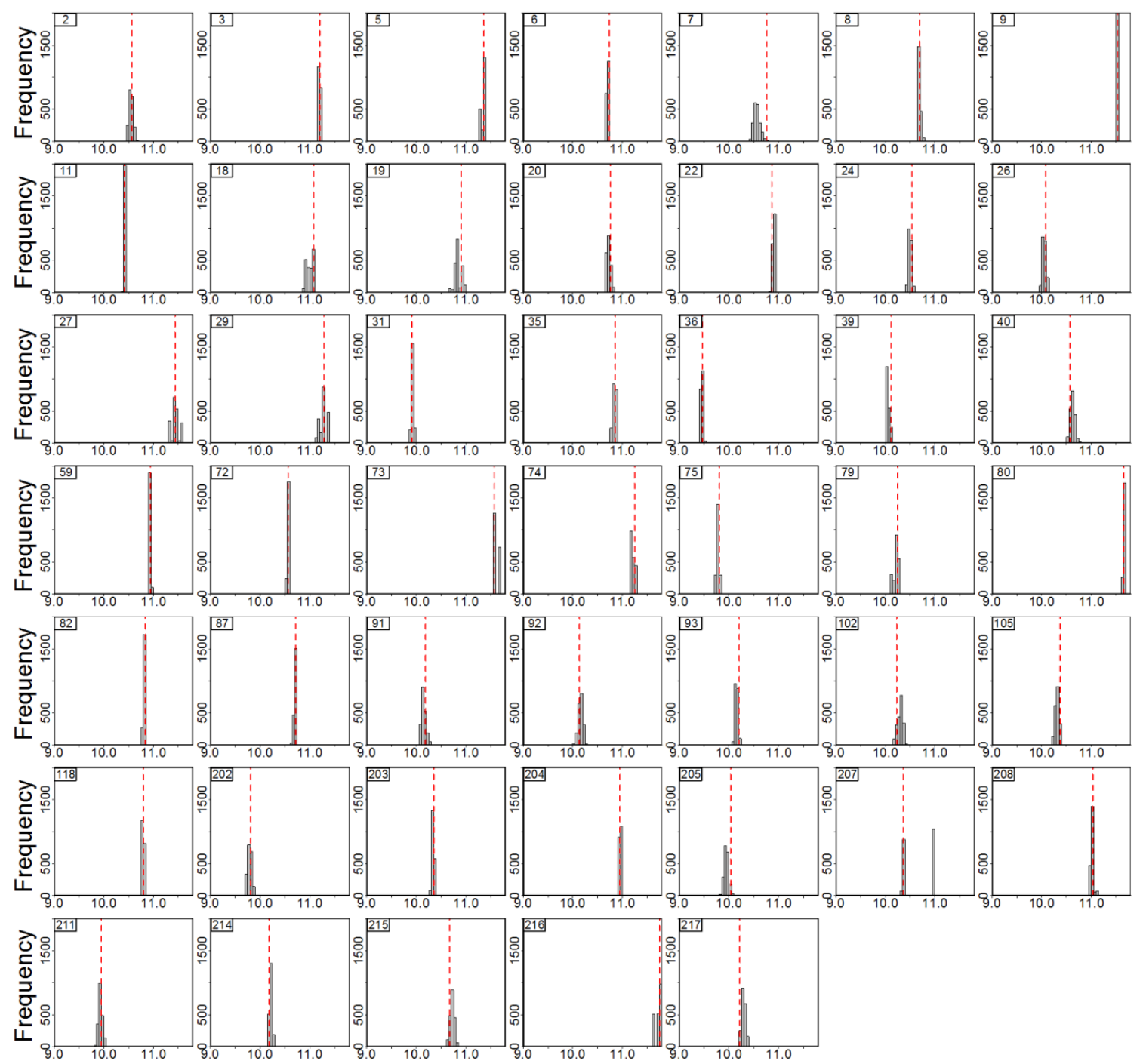

MFS, $\ln \left(m^{2}\right) \quad$ MFS, $\ln \left(m^{2}\right) \quad$ MFS, $\ln \left(m^{2}\right) \quad$ MFS, $\ln \left(m^{2}\right) \quad M F S, \ln \left(m^{2}\right) \quad M F S, \ln \left(m^{2}\right) \quad M F S, \ln \left(m^{2}\right)$

Figure K.3 MFS metric distributions for all year-2012 LCs. The distributions are labeled in the upper left corner, with numbers corresponding to the Farmland Biodiversity Project landscape numbering system. The dashed red line represents the hard metric. 

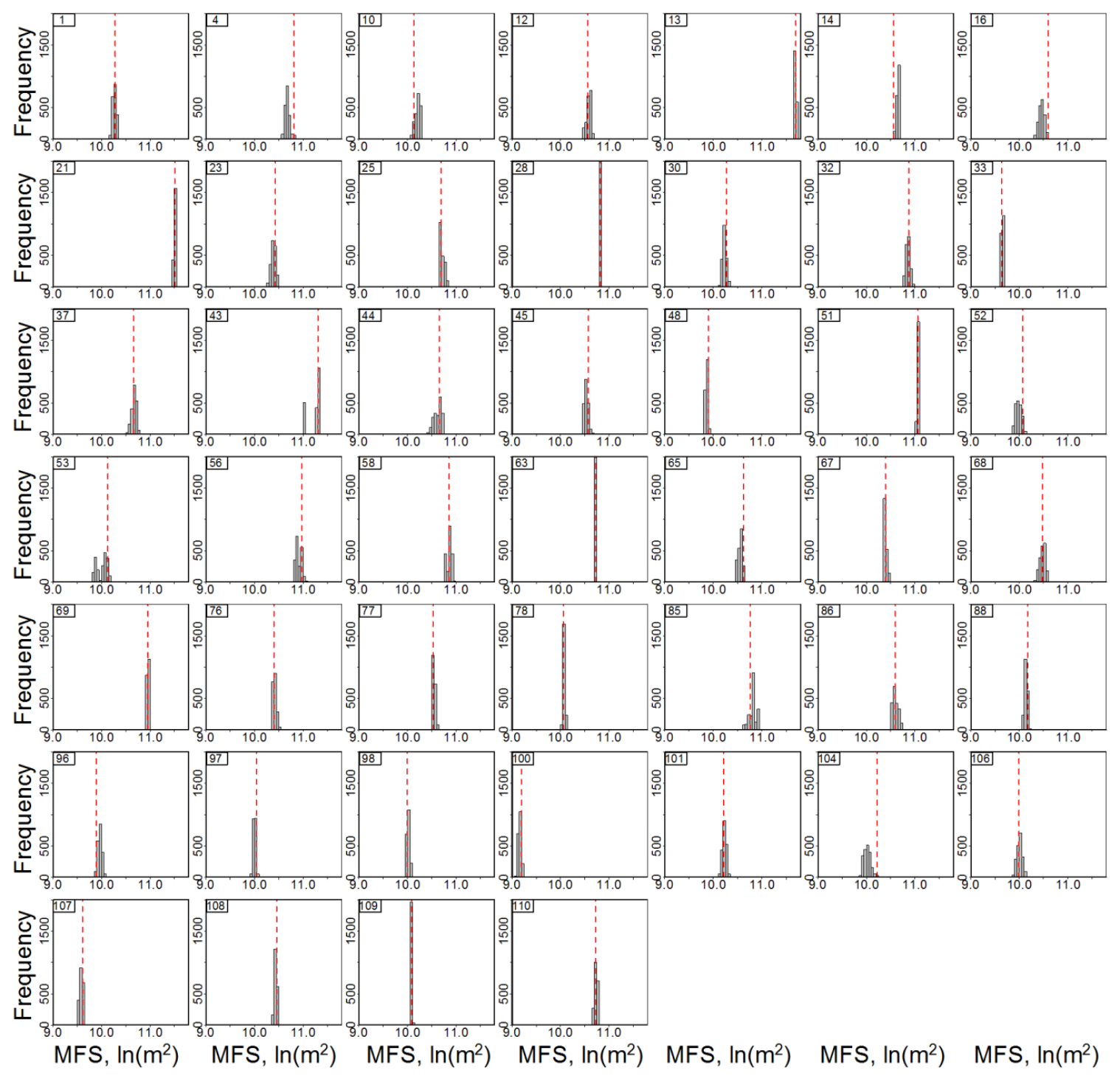

Figure K.4 MFS metric distributions for all year-2011 LCs. The distributions are labeled in the upper left corner, with numbers corresponding to the Farmland Biodiversity Project landscape numbering system. The dashed red line represents the hard metric. 

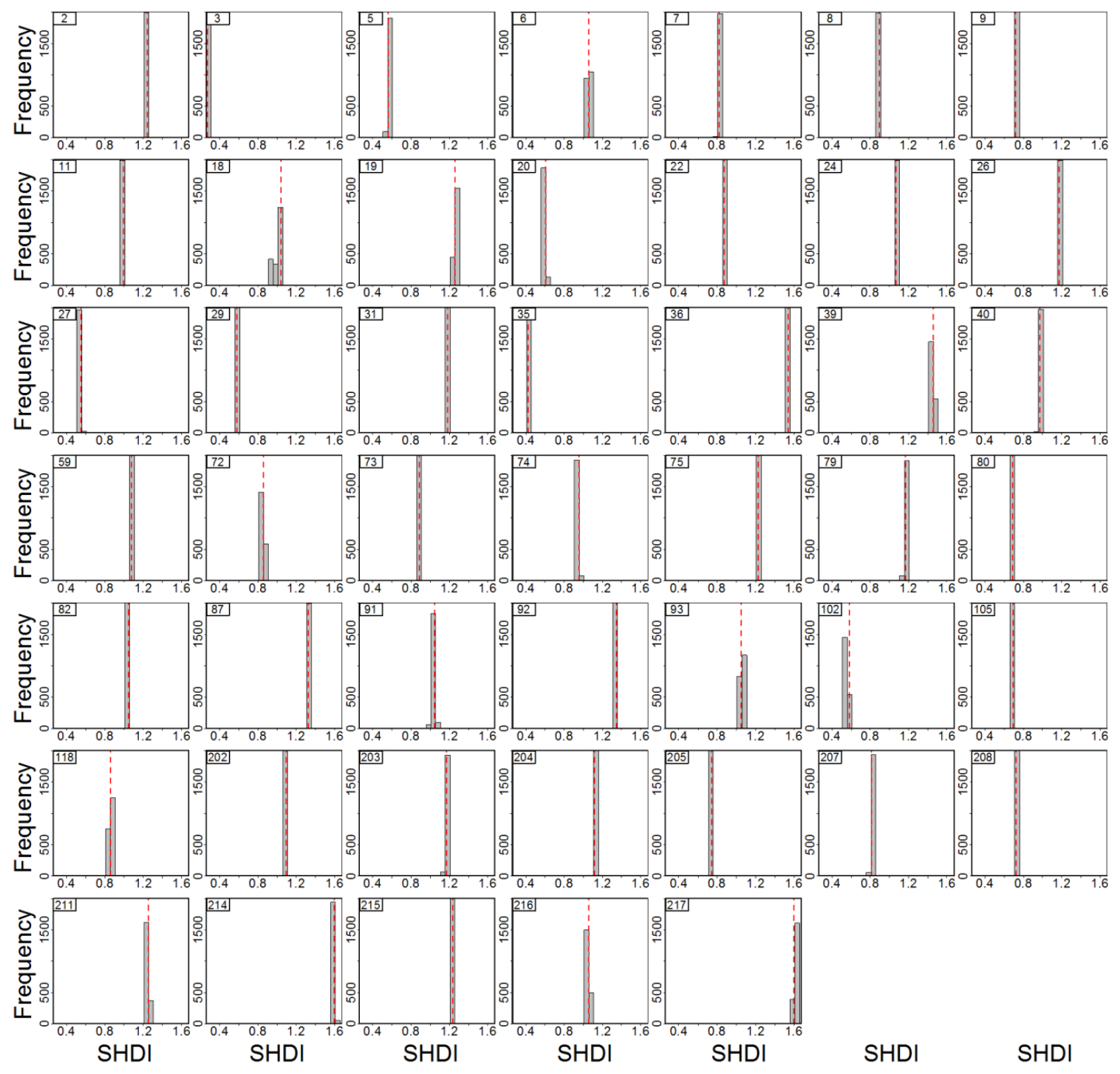

Figure K.5 SHDI metric distributions for all year-2012 LCs. These distributions were not influenced by thematic uncertainty associated with the year-2011 image classification, however, they were influenced slightly by changes in field area due to sensitized vertices, or the removal of fields from consideration (boundary interpretation uncertainty). The distributions are labeled in the upper left corner, with numbers corresponding to the Farmland Biodiversity Project landscape numbering system. The dashed red line represents the hard metric. 

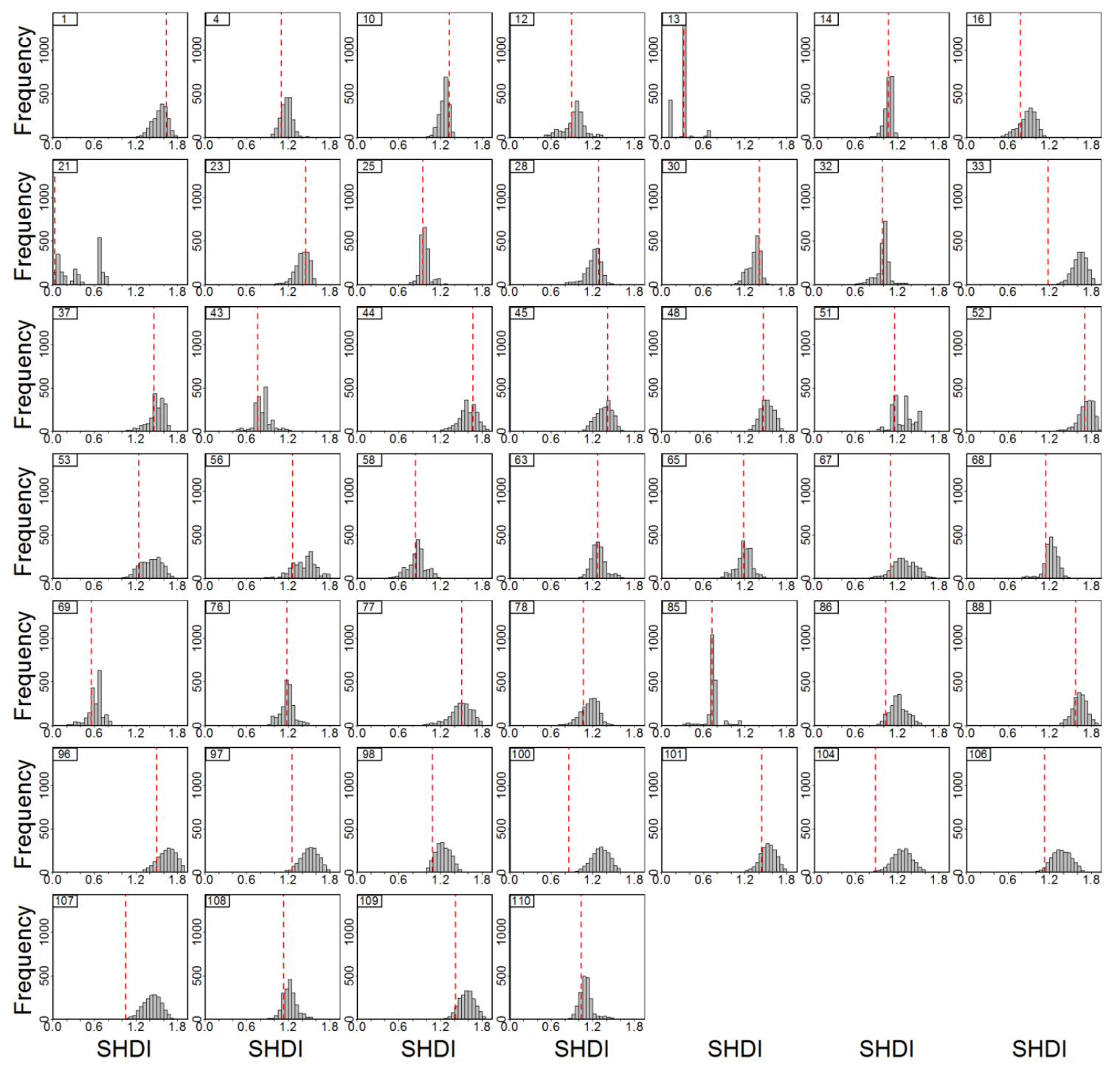

Figure K.6 SHDI metric distributions for all year-2011 LCs. These distributions were calculated under the independent field simulation approach. The distributions are labeled in the upper left corner, with numbers corresponding to the Farmland Biodiversity Project landscape numbering system. The dashed red line represents the hard metric. 

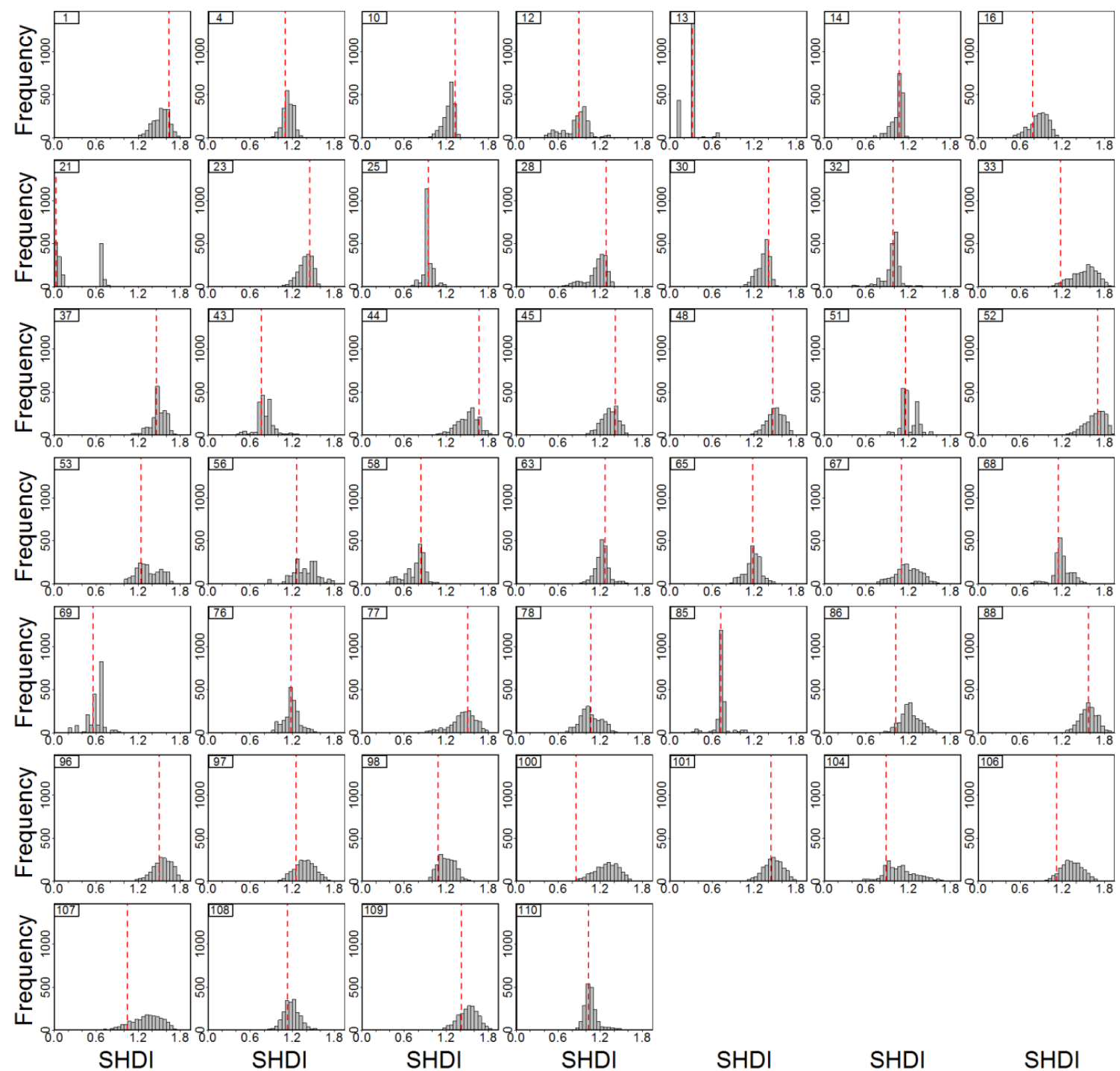

SHDI SHDI

SHDI

Figure K.7 SHDI metric distributions for all year-2011 LCs. These distributions were calculated under the locally-grouped simulation approach. The distributions are labeled in the upper left corner, with numbers corresponding to the Farmland Biodiversity Project landscape numbering system. The dashed red line represents the hard metric. 

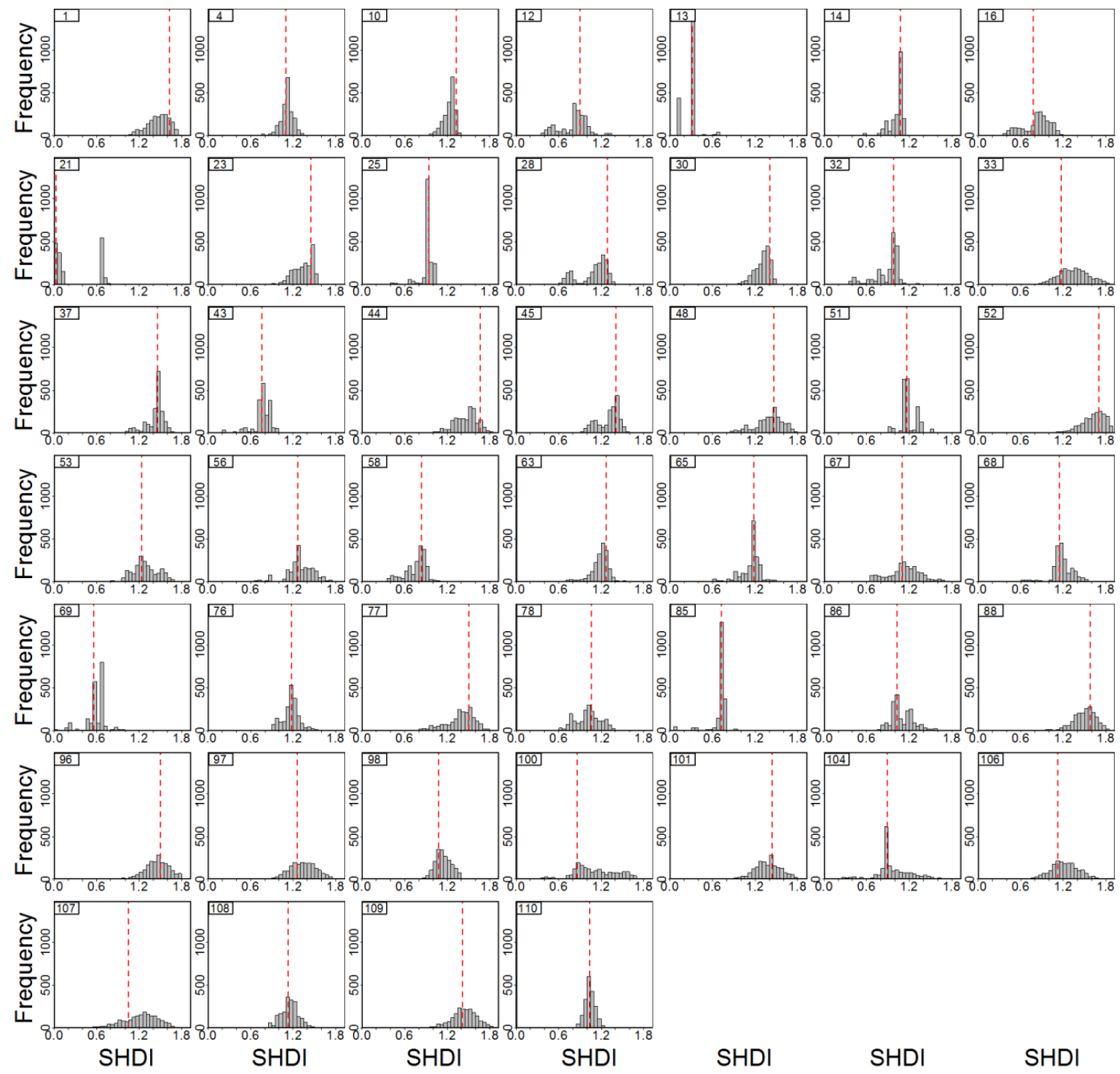

Figure K.8 SHDI metric distributions for all year-2011 LCs. These distributions were calculated under the landscape-grouped simulation approach. The distributions are labeled in the upper left corner, with numbers corresponding to the Farmland Biodiversity Project landscape numbering system. The dashed red line represents the hard metric. 


\section{Appendix L: Moran's I statistics}

A brief check for spatial autocorrelation in the per-field class membership probabilities was performed, using global Moran's I statistics. Moran's I was calculated (using ArcGIS 10.2) for each set of class membership probabilities (inverse distance relationship; row standardized, to account for aggregation to the field level; distance threshold of 1414 meters). The Moran's I statistics are provided in the table below.

Table L.1 Moran's I index for the per-field class membership probabilities.

\begin{tabular}{ccc}
\hline Class & Moran's Index & $\begin{array}{c}\text { Significance of } \\
\text { clustering, relative to } \\
\text { random }\end{array}$ \\
\hline Corn & 0.123 & $\mathrm{p}<0.001$ \\
Soybean & 0.218 & $\mathrm{p}<0.001$ \\
Hay & 0.186 & $\mathrm{p}<0.001$ \\
Pasture & 0.205 & $\mathrm{p}<0.001$ \\
Legumes & 0.072 & $\mathrm{p}<0.001$ \\
Cereals & 0.033 & $\mathrm{p}=0.007$ \\
Fallow & 0.182 & $\mathrm{p}<0.001$ \\
\hline
\end{tabular}




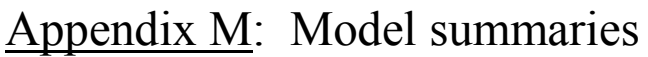

Table M.1 Model parameter details for the beetles, birds, and butterflies taxa. Simulated values are based on independent field simulation.

\begin{tabular}{|c|c|c|c|c|c|c|c|}
\hline Taxon & Diversity & Variable & $\widehat{\bar{\theta}}_{\text {hard }}$ & $\widehat{\operatorname{se}}\left(\widehat{\bar{\theta}}_{\text {hard }}\right)$ & $\overline{\bar{\theta}}_{\text {sim }}$ & $\widehat{\operatorname{se}}\left(\widehat{\bar{\theta}}_{\operatorname{sim}}\right)$ & $\sigma_{\operatorname{sim}}$ \\
\hline Beetles & Gamma & Intercept & 5.63344 & 0.43545 & 5.58185 & 0.44141 & 0.03264 \\
\hline Beetles & Gamma & P.AG & -0.94083 & 0.38060 & -0.98230 & 0.37810 & 0.03699 \\
\hline Beetles & Gamma & MFS & -0.57537 & 0.45022 & -0.49200 & 0.48389 & 0.10281 \\
\hline Beetles & Gamma & SHDI & 0.26849 & 0.33173 & 0.27363 & 0.35701 & 0.12542 \\
\hline Beetles & Gamma & Year & 0.70900 & 0.66218 & 0.80916 & 0.67719 & 0.06236 \\
\hline Beetles & Beta & Intercept & 2.70876 & 0.26747 & 2.67046 & 0.27111 & 0.02144 \\
\hline Beetles & Beta & P.AG & -0.70850 & 0.22644 & -0.73357 & 0.22799 & 0.02135 \\
\hline Beetles & Beta & MFS & -0.23258 & 0.30386 & -0.16212 & 0.32217 & 0.07768 \\
\hline Beetles & Beta & SHDI & 0.24326 & 0.19464 & 0.24687 & 0.21051 & 0.06963 \\
\hline Beetles & Beta & Year & 0.31103 & 0.40468 & 0.38548 & 0.41426 & 0.04119 \\
\hline Beetles & Alpha & Intercept & 2.91601 & 0.18774 & 2.90763 & 0.19130 & 0.01010 \\
\hline Beetles & Alpha & P.AG & -0.23184 & 0.17738 & -0.24571 & 0.17686 & 0.01960 \\
\hline Beetles & Alpha & MFS & -0.27167 & 0.15666 & -0.26004 & 0.16745 & 0.02832 \\
\hline Beetles & Alpha & SHDI & 0.01861 & 0.15915 & 0.02003 & 0.17465 & 0.07127 \\
\hline Beetles & Alpha & Year & 0.41465 & 0.27863 & 0.43104 & 0.28719 & 0.01892 \\
\hline Beetles & Abund. & Intercept & 101.83312 & 19.00459 & 103.06942 & 19.34791 & 1.19178 \\
\hline Beetles & Abund. & P.AG & 1.67640 & 21.51735 & 3.64007 & 21.52307 & 3.48151 \\
\hline Beetles & Abund. & MFS & 30.99761 & 15.72543 & 30.13307 & 16.36311 & 2.43053 \\
\hline Beetles & Abund. & SHDI & -14.69760 & 16.39867 & -15.09473 & 17.96617 & 6.64065 \\
\hline Beetles & Abund. & Year & 31.11234 & 27.69229 & 28.66159 & 28.51440 & 2.20488 \\
\hline Birds & Gamma & Intercept & 6.40541 & 0.39325 & 6.40946 & 0.39971 & 0.02528 \\
\hline Birds & Gamma & P.AG & -0.76422 & 0.33225 & -0.71321 & 0.34769 & 0.03458 \\
\hline Birds & Gamma & MFS & -0.43971 & 0.38512 & -0.48997 & 0.38517 & 0.06534 \\
\hline Birds & Gamma & SHDI & 0.12662 & 0.29868 & 0.20958 & 0.32923 & 0.11446 \\
\hline Birds & Gamma & Year & 0.53066 & 0.59452 & 0.52420 & 0.61061 & 0.04992 \\
\hline Birds & Beta & Intercept & 3.88851 & 0.26905 & 3.89407 & 0.27338 & 0.02045 \\
\hline Birds & Beta & P.AG & -0.53457 & 0.24179 & -0.48919 & 0.25406 & 0.02932 \\
\hline Birds & Beta & MFS & -0.38916 & 0.25831 & -0.42437 & 0.25623 & 0.04203 \\
\hline Birds & Beta & SHDI & 0.09358 & 0.21054 & 0.14501 & 0.23256 & 0.08332 \\
\hline Birds & Beta & Year & 0.54993 & 0.40868 & 0.54012 & 0.41957 & 0.04049 \\
\hline Birds & Alpha & Intercept & 2.53272 & 0.15160 & 2.53086 & 0.15285 & 0.00637 \\
\hline Birds & Alpha & P.AG & -0.21466 & 0.12289 & -0.20595 & 0.12615 & 0.01090 \\
\hline Birds & Alpha & MFS & -0.06689 & 0.14350 & -0.07784 & 0.14576 & 0.02407 \\
\hline Birds & Alpha & SHDI & 0.02701 & 0.11199 & 0.05770 & 0.12141 & 0.04076 \\
\hline Birds & Alpha & Year & -0.05023 & 0.22689 & -0.04621 & 0.23016 & 0.01255 \\
\hline Birds & Abund. & Intercept & 16.21070 & 1.01088 & 16.16996 & 1.01674 & 0.04135 \\
\hline Birds & Abund. & P.AG & -1.14229 & 0.84596 & -1.10530 & 0.88005 & 0.08102 \\
\hline Birds & Abund. & MFS & -0.78653 & 0.85855 & -0.90295 & 0.87350 & 0.13006 \\
\hline Birds & Abund. & SHDI & 0.19461 & 0.77073 & 0.42471 & 0.84063 & 0.29138 \\
\hline Birds & Abund. & Year & -1.76146 & 1.49843 & -1.68044 & 1.51709 & 0.08237 \\
\hline Butterflies & Gamma & Intercept & 3.81163 & 0.42486 & 3.78922 & 0.43152 & 0.01903 \\
\hline Butterflies & Gamma & P.AG & 0.10302 & 0.45725 & 0.05901 & 0.48047 & 0.07960 \\
\hline Butterflies & Gamma & MFS & -1.29118 & 0.35185 & -1.21266 & 0.34897 & 0.04450 \\
\hline Butterflies & Gamma & SHDI & -0.33009 & 0.35154 & -0.04611 & 0.40787 & 0.14751 \\
\hline Butterflies & Gamma & Year & 0.60316 & 0.60901 & 0.64399 & 0.62345 & 0.03670 \\
\hline Butterflies & Beta & Intercept & 2.05608 & 0.24387 & 2.04604 & 0.24737 & 0.01519 \\
\hline Butterflies & Beta & P.AG & 0.14291 & 0.25833 & 0.11331 & 0.26679 & 0.04279 \\
\hline Butterflies & Beta & MFS & -0.78570 & 0.21606 & -0.73986 & 0.21028 & 0.03404 \\
\hline Butterflies & Beta & SHDI & -0.26052 & 0.19911 & -0.15653 & 0.22684 & 0.08073 \\
\hline Butterflies & Beta & Year & 0.36212 & 0.35259 & 0.37956 & 0.36011 & 0.02927 \\
\hline Butterflies & Alpha & Intercept & 1.75920 & 0.21107 & 1.73859 & 0.21758 & 0.01329 \\
\hline Butterflies & Alpha & P.AG & -0.05955 & 0.24160 & -0.09319 & 0.24824 & 0.04772 \\
\hline Butterflies & Alpha & MFS & -0.52007 & 0.16660 & -0.47915 & 0.17479 & 0.02395 \\
\hline Butterflies & Alpha & SHDI & -0.06150 & 0.18073 & 0.13972 & 0.20984 & 0.07921 \\
\hline Butterflies & Alpha & Year & 0.23415 & 0.30229 & 0.27319 & 0.31786 & 0.02552 \\
\hline Butterflies & Abund. & Intercept & 16.90563 & 2.88286 & 16.12222 & 2.98355 & 0.39426 \\
\hline Butterflies & Abund. & P.AG & 2.62098 & 3.18174 & 1.79254 & 3.22846 & 0.64870 \\
\hline Butterflies & Abund. & MFS & -5.80114 & 2.50810 & -4.60525 & 2.84780 & 0.64711 \\
\hline Butterflies & Abund. & SHDI & 1.52726 & 2.52047 & 4.47330 & 2.52302 & 0.89443 \\
\hline Butterflies & Abund. & Year & 1.28218 & 4.20153 & 2.78798 & 4.46118 & 0.75763 \\
\hline
\end{tabular}


Table M.2 Model parameter details for the plants, spiders, and syrphids taxa. Simulated values are based on independent field simulation.

\begin{tabular}{|c|c|c|c|c|c|c|c|}
\hline Taxon & Diversity & Variable & $\widehat{\bar{\Theta}}_{\text {hard }}$ & $\widehat{\operatorname{se}}\left(\widehat{\bar{\theta}}_{\text {hard }}\right)$ & $\widehat{\bar{\theta}}_{\text {sim }}$ & $\widehat{\operatorname{se}}\left(\widehat{\bar{\Theta}}_{\operatorname{sim}}\right)$ & $\sigma_{\text {sim }}$ \\
\hline Plants & Gamma & Intercept & 36.93728 & 1.74601 & 36.90300 & 1.74003 & 0.09037 \\
\hline Plants & Gamma & P.AG & -1.50311 & 1.82609 & -0.98570 & 1.86656 & 0.33413 \\
\hline Plants & Gamma & MFS & -7.14049 & 1.48271 & -7.37555 & 1.46986 & 0.20678 \\
\hline Plants & Gamma & SHDI & 0.12493 & 1.46245 & 0.29328 & 1.60446 & 0.60531 \\
\hline Plants & Gamma & Year & -11.36525 & 2.54787 & -11.29742 & 2.54809 & 0.17881 \\
\hline Plants & Beta & Intercept & 21.83327 & 1.06045 & 21.78969 & 1.06615 & 0.06722 \\
\hline Plants & Beta & P.AG & -0.38279 & 1.16016 & -0.02248 & 1.17164 & 0.22309 \\
\hline Plants & Beta & MFS & -3.86727 & 0.88465 & -3.99838 & 0.90510 & 0.14761 \\
\hline Plants & Beta & SHDI & 0.73247 & 0.89698 & 0.69300 & 0.98563 & 0.38506 \\
\hline Plants & Beta & Year & -6.48392 & 1.53658 & -6.39769 & 1.56186 & 0.13300 \\
\hline Plants & Alpha & Intercept & 15.03345 & 0.78950 & 15.04811 & 0.78683 & 0.05279 \\
\hline Plants & Alpha & P.AG & -1.16635 & 0.79198 & -1.00006 & 0.80862 & 0.14410 \\
\hline Plants & Alpha & MFS & -3.15056 & 0.77954 & -3.26424 & 0.74408 & 0.13764 \\
\hline Plants & Alpha & SHDI & -0.57426 & 0.63972 & -0.36422 & 0.70746 & 0.25984 \\
\hline Plants & Alpha & Year & -4.74171 & 1.18841 & -4.77073 & 1.18498 & 0.10445 \\
\hline Spiders & Gamma & Intercept & 5.16664 & 0.39490 & 5.10035 & 0.40886 & 0.05170 \\
\hline Spiders & Gamma & P.AG & -0.73537 & 0.42194 & -0.76351 & 0.40649 & 0.07646 \\
\hline Spiders & Gamma & MFS & -0.86561 & 0.37696 & -0.79946 & 0.40425 & 0.09162 \\
\hline Spiders & Gamma & SHDI & -0.05559 & 0.34756 & 0.31193 & 0.35848 & 0.14463 \\
\hline Spiders & Gamma & Year & -1.64768 & 0.59980 & -1.52095 & 0.63269 & 0.09742 \\
\hline Spiders & Beta & Intercept & 2.75176 & 0.22361 & 2.72238 & 0.23178 & 0.02680 \\
\hline Spiders & Beta & P.AG & -0.34707 & 0.24985 & -0.35243 & 0.24604 & 0.05175 \\
\hline Spiders & Beta & MFS & -0.54204 & 0.20134 & -0.52334 & 0.21408 & 0.04499 \\
\hline Spiders & Beta & SHDI & 0.00123 & 0.19900 & 0.17973 & 0.21405 & 0.08461 \\
\hline Spiders & Beta & Year & -1.11445 & 0.33545 & -1.05772 & 0.35492 & 0.05026 \\
\hline Spiders & Alpha & Intercept & 2.41241 & 0.19489 & 2.38205 & 0.19848 & 0.01904 \\
\hline Spiders & Alpha & P.AG & -0.39603 & 0.18484 & -0.40696 & 0.17754 & 0.02370 \\
\hline Spiders & Alpha & MFS & -0.33300 & 0.19370 & -0.27978 & 0.20699 & 0.04619 \\
\hline Spiders & Alpha & SHDI & -0.05105 & 0.16627 & 0.12177 & 0.16554 & 0.06802 \\
\hline Spiders & Alpha & Year & -0.52863 & 0.29668 & -0.47088 & 0.30539 & 0.03610 \\
\hline Spiders & Abund. & Intercept & 19.96763 & 2.27490 & 19.50444 & 2.30852 & 0.20206 \\
\hline Spiders & Abund. & P.AG & -5.00481 & 2.27496 & -5.38836 & 2.13610 & 0.23714 \\
\hline Spiders & Abund. & MFS & -4.31533 & 2.36360 & -3.52533 & 2.47301 & 0.46388 \\
\hline Spiders & Abund. & SHDI & -0.90394 & 1.98015 & 1.20934 & 1.95206 & 0.77900 \\
\hline Spiders & Abund. & Year & -1.37658 & 3.48382 & -0.49555 & 3.56536 & 0.38358 \\
\hline Syrphids & Gamma & Intercept & 3.21300 & 0.28833 & 3.15501 & 0.29301 & 0.03105 \\
\hline Syrphids & Gamma & P.AG & 0.25698 & 0.32194 & 0.12071 & 0.32029 & 0.04808 \\
\hline Syrphids & Gamma & MFS & -0.52562 & 0.25192 & -0.41918 & 0.25652 & 0.04453 \\
\hline Syrphids & Gamma & SHDI & 0.01870 & 0.25314 & 0.29889 & 0.25320 & 0.10832 \\
\hline Syrphids & Gamma & Year & -1.98513 & 0.42058 & -1.87419 & 0.43181 & 0.05968 \\
\hline Syrphids & Beta & Intercept & 1.81542 & 0.16990 & 1.78891 & 0.17205 & 0.01463 \\
\hline Syrphids & Beta & P.AG & 0.12539 & 0.19175 & 0.05691 & 0.18729 & 0.02672 \\
\hline Syrphids & Beta & MFS & -0.27943 & 0.14678 & -0.23334 & 0.14689 & 0.02192 \\
\hline Syrphids & Beta & SHDI & 0.00673 & 0.14807 & 0.13824 & 0.14961 & 0.06369 \\
\hline Syrphids & Beta & Year & -1.06692 & 0.24709 & -1.01624 & 0.25237 & 0.02813 \\
\hline Syrphids & Alpha & Intercept & 1.39505 & 0.13350 & 1.36327 & 0.13622 & 0.01677 \\
\hline Syrphids & Alpha & P.AG & 0.11307 & 0.14945 & 0.04935 & 0.14587 & 0.02116 \\
\hline Syrphids & Alpha & MFS & -0.24301 & 0.11556 & -0.18284 & 0.12165 & 0.02492 \\
\hline Syrphids & Alpha & SHDI & 0.02440 & 0.11701 & 0.16449 & 0.11433 & 0.04786 \\
\hline Syrphids & Alpha & Year & -0.91343 & 0.19440 & -0.85254 & 0.20113 & 0.03223 \\
\hline Syrphids & Abund. & Intercept & 11.54142 & 1.47406 & 11.47080 & 1.50038 & 0.09161 \\
\hline Syrphids & Abund. & P.AG & -1.54393 & 1.35576 & -1.59382 & 1.28363 & 0.12872 \\
\hline Syrphids & Abund. & MFS & -1.98431 & 1.22445 & -1.50751 & 1.25517 & 0.20793 \\
\hline Syrphids & Abund. & SHDI & -0.88370 & 1.23723 & 0.02112 & 1.28893 & 0.63635 \\
\hline Syrphids & Abund. & Year & -7.79159 & 2.15908 & -7.66003 & 2.21957 & 0.17638 \\
\hline
\end{tabular}


Table M.3 Model parameter details for the beetles, birds, and butterflies taxa. Simulated values are based on locally-grouped simulation.

\begin{tabular}{|c|c|c|c|c|c|c|c|}
\hline Taxon & Diversity & Variable & $\widehat{\widehat{\theta}}_{\text {hard }}$ & $\widehat{\operatorname{se}}\left(\widehat{\bar{\Theta}}_{\text {hard }}\right)$ & $\widehat{\bar{\theta}}_{\text {sim }}$ & $\widehat{\operatorname{se}}\left(\widehat{\bar{\Theta}}_{\operatorname{sim}}\right)$ & $\sigma_{\text {sim }}$ \\
\hline Beetles & Gamma & Intercept & 5.63344 & 0.43545 & 5.58958 & 0.43850 & 0.03052 \\
\hline Beetles & Gamma & P.AG & -0.94083 & 0.38060 & -0.98548 & 0.37809 & 0.03607 \\
\hline Beetles & Gamma & MFS & -0.57537 & 0.45022 & -0.49513 & 0.48192 & 0.10384 \\
\hline Beetles & Gamma & SHDI & 0.26849 & 0.33173 & 0.24434 & 0.34725 & 0.13834 \\
\hline Beetles & Gamma & Year & 0.70900 & 0.66218 & 0.79408 & 0.67013 & 0.05800 \\
\hline Beetles & Beta & Intercept & 2.70876 & 0.26747 & 2.67935 & 0.26872 & 0.01912 \\
\hline Beetles & Beta & P.AG & -0.70850 & 0.22644 & -0.73647 & 0.22774 & 0.02047 \\
\hline Beetles & Beta & MFS & -0.23258 & 0.30386 & -0.16904 & 0.31809 & 0.07813 \\
\hline Beetles & Beta & SHDI & 0.24326 & 0.19464 & 0.22713 & 0.20433 & 0.07387 \\
\hline Beetles & Beta & Year & 0.31103 & 0.40468 & 0.36813 & 0.40839 & 0.03649 \\
\hline Beetles & Alpha & Intercept & 2.91601 & 0.18774 & 2.90846 & 0.19045 & 0.00933 \\
\hline Beetles & Alpha & P.AG & -0.23184 & 0.17738 & -0.24617 & 0.17696 & 0.01933 \\
\hline Beetles & Alpha & MFS & -0.27167 & 0.15666 & -0.26064 & 0.16761 & 0.02925 \\
\hline Beetles & Alpha & SHDI & 0.01861 & 0.15915 & 0.01090 & 0.16850 & 0.07952 \\
\hline Beetles & Alpha & Year & 0.41465 & 0.27863 & 0.42947 & 0.28513 & 0.01733 \\
\hline Beetles & Abund. & Intercept & 101.83312 & 19.00459 & 102.44509 & 19.18521 & 0.90785 \\
\hline Beetles & Abund. & P.AG & 1.67640 & 21.51735 & 3.60055 & 21.65682 & 3.54165 \\
\hline Beetles & Abund. & MFS & 30.99761 & 15.72543 & 30.45368 & 16.21162 & 2.40838 \\
\hline Beetles & Abund. & SHDI & -14.69760 & 16.39867 & -13.04303 & 17.50372 & 6.72453 \\
\hline Beetles & Abund. & Year & 31.11234 & 27.69229 & 29.86067 & 28.11819 & 1.66048 \\
\hline Birds & Gamma & Intercept & 6.40541 & 0.39325 & 6.41764 & 0.39766 & 0.02478 \\
\hline Birds & Gamma & P.AG & -0.76422 & 0.33225 & -0.71427 & 0.34870 & 0.03557 \\
\hline Birds & Gamma & MFS & -0.43971 & 0.38512 & -0.49822 & 0.38278 & 0.06893 \\
\hline Birds & Gamma & SHDI & 0.12662 & 0.29868 & 0.16423 & 0.32112 & 0.13272 \\
\hline Birds & Gamma & Year & 0.53066 & 0.59452 & 0.50788 & 0.60518 & 0.04888 \\
\hline Birds & Beta & Intercept & 3.88851 & 0.26905 & 3.89955 & 0.27153 & 0.01992 \\
\hline Birds & Beta & P.AG & -0.53457 & 0.24179 & -0.48964 & 0.25504 & 0.03017 \\
\hline Birds & Beta & MFS & -0.38916 & 0.25831 & -0.42792 & 0.25517 & 0.04453 \\
\hline Birds & Beta & SHDI & 0.09358 & 0.21054 & 0.11775 & 0.22628 & 0.09603 \\
\hline Birds & Beta & Year & 0.54993 & 0.40868 & 0.52921 & 0.41473 & 0.03941 \\
\hline Birds & Alpha & Intercept & 2.53272 & 0.15160 & 2.53334 & 0.15244 & 0.00629 \\
\hline Birds & Alpha & P.AG & -0.21466 & 0.12289 & -0.20621 & 0.12606 & 0.01122 \\
\hline Birds & Alpha & MFS & -0.06689 & 0.14350 & -0.08218 & 0.14469 & 0.02522 \\
\hline Birds & Alpha & SHDI & 0.02701 & 0.11199 & 0.03998 & 0.11829 & 0.04758 \\
\hline Birds & Alpha & Year & -0.05023 & 0.22689 & -0.05119 & 0.22903 & 0.01237 \\
\hline Birds & Abund. & Intercept & 16.21070 & 1.01088 & 16.18424 & 1.01270 & 0.03865 \\
\hline Birds & Abund. & P.AG & -1.14229 & 0.84596 & -1.10417 & 0.88120 & 0.08200 \\
\hline Birds & Abund. & MFS & -0.78653 & 0.85855 & -0.91318 & 0.87156 & 0.13911 \\
\hline Birds & Abund. & SHDI & 0.19461 & 0.77073 & 0.36971 & 0.81713 & 0.33686 \\
\hline Birds & Abund. & Year & -1.76146 & 1.49843 & -1.70888 & 1.50618 & 0.07704 \\
\hline Butterflies & Gamma & Intercept & 3.81163 & 0.42486 & 3.79237 & 0.42944 & 0.01963 \\
\hline Butterflies & Gamma & P.AG & 0.10302 & 0.45725 & 0.06930 & 0.47974 & 0.08325 \\
\hline Butterflies & Gamma & MFS & -1.29118 & 0.35185 & -1.22344 & 0.35065 & 0.05108 \\
\hline Butterflies & Gamma & SHDI & -0.33009 & 0.35154 & -0.10003 & 0.38937 & 0.16684 \\
\hline Butterflies & Gamma & Year & 0.60316 & 0.60901 & 0.63800 & 0.61817 & 0.03778 \\
\hline Butterflies & Beta & Intercept & 2.05608 & 0.24387 & 2.04370 & 0.24570 & 0.01552 \\
\hline Butterflies & Beta & P.AG & 0.14291 & 0.25833 & 0.11773 & 0.26712 & 0.04663 \\
\hline Butterflies & Beta & MFS & -0.78570 & 0.21606 & -0.74404 & 0.21109 & 0.03965 \\
\hline Butterflies & Beta & SHDI & -0.26052 & 0.19911 & -0.15982 & 0.21769 & 0.09138 \\
\hline Butterflies & Beta & Year & 0.36212 & 0.35259 & 0.38422 & 0.35589 & 0.02981 \\
\hline Butterflies & Alpha & Intercept & 1.75920 & 0.21107 & 1.74689 & 0.21501 & 0.01106 \\
\hline Butterflies & Alpha & P.AG & -0.05955 & 0.24160 & -0.08305 & 0.24927 & 0.04889 \\
\hline Butterflies & Alpha & MFS & -0.52007 & 0.16660 & -0.48739 & 0.17257 & 0.02461 \\
\hline Butterflies & Alpha & SHDI & -0.06150 & 0.18073 & 0.08294 & 0.20074 & 0.09169 \\
\hline Butterflies & Alpha & Year & 0.23415 & 0.30229 & 0.25712 & 0.31126 & 0.02118 \\
\hline Butterflies & Abund. & Intercept & 16.90563 & 2.88286 & 16.48494 & 2.93001 & 0.35824 \\
\hline Butterflies & Abund. & P.AG & 2.62098 & 3.18174 & 1.99695 & 3.26330 & 0.70673 \\
\hline Butterflies & Abund. & MFS & -5.80114 & 2.50810 & -4.85394 & 2.77636 & 0.69038 \\
\hline Butterflies & Abund. & SHDI & 1.52726 & 2.52047 & 3.76509 & 2.49599 & 1.20331 \\
\hline Butterflies & Abund. & Year & 1.28218 & 4.20153 & 2.08236 & 4.31975 & 0.68369 \\
\hline
\end{tabular}


Table M.4 Model parameter details for the plants, spiders, and syrphids taxa.

Simulated values are based on locally-grouped simulation.

\begin{tabular}{|c|c|c|c|c|c|c|c|}
\hline Taxon & Diversity & Variable & $\widehat{\bar{\theta}}_{\text {hard }}$ & $\widehat{\operatorname{se}}\left(\widehat{\bar{\Theta}}_{\text {hard }}\right)$ & $\widehat{\bar{\theta}}_{\text {sim }}$ & $\widehat{\operatorname{se}}\left(\widehat{\bar{\Theta}}_{\operatorname{sim}}\right)$ & $\sigma_{\text {sim }}$ \\
\hline Plants & Gamma & Intercept & 36.93728 & 1.74601 & 36.92239 & 1.73074 & 0.08922 \\
\hline Plants & Gamma & P.AG & -1.50311 & 1.82609 & -0.96691 & 1.86154 & 0.34194 \\
\hline Plants & Gamma & MFS & -7.14049 & 1.48271 & -7.41705 & 1.46086 & 0.21454 \\
\hline Plants & Gamma & SHDI & 0.12493 & 1.46245 & 0.04703 & 1.54174 & 0.62157 \\
\hline Plants & Gamma & Year & -11.36525 & 2.54787 & -11.33579 & 2.52312 & 0.17655 \\
\hline Plants & Beta & Intercept & 21.83327 & 1.06045 & 21.81184 & 1.05771 & 0.05901 \\
\hline Plants & Beta & P.AG & -0.38279 & 1.16016 & -0.01716 & 1.16861 & 0.22775 \\
\hline Plants & Beta & MFS & -3.86727 & 0.88465 & -4.01654 & 0.89541 & 0.14692 \\
\hline Plants & Beta & SHDI & 0.73247 & 0.89698 & 0.60369 & 0.94751 & 0.39379 \\
\hline Plants & Beta & Year & -6.48392 & 1.53658 & -6.44152 & 1.53904 & 0.11677 \\
\hline Plants & Alpha & Intercept & 15.03345 & 0.78950 & 15.06020 & 0.78218 & 0.05957 \\
\hline Plants & Alpha & P.AG & -1.16635 & 0.79198 & -0.97855 & 0.80952 & 0.15347 \\
\hline Plants & Alpha & MFS & -3.15056 & 0.77954 & -3.31762 & 0.75371 & 0.16426 \\
\hline Plants & Alpha & SHDI & -0.57426 & 0.63972 & -0.52702 & 0.67694 & 0.27955 \\
\hline Plants & Alpha & Year & -4.74171 & 1.18841 & -4.79466 & 1.17358 & 0.11787 \\
\hline Spiders & Gamma & Intercept & 5.16664 & 0.39490 & 5.12718 & 0.40214 & 0.04716 \\
\hline Spiders & Gamma & P.AG & -0.73537 & 0.42194 & -0.75136 & 0.41517 & 0.08308 \\
\hline Spiders & Gamma & MFS & -0.86561 & 0.37696 & -0.82739 & 0.39456 & 0.09121 \\
\hline Spiders & Gamma & SHDI & -0.05559 & 0.34756 & 0.19490 & 0.35453 & 0.18001 \\
\hline Spiders & Gamma & Year & -1.64768 & 0.59980 & -1.57185 & 0.61648 & 0.08839 \\
\hline Spiders & Beta & Intercept & 2.75176 & 0.22361 & 2.73542 & 0.22736 & 0.02305 \\
\hline Spiders & Beta & P.AG & -0.34707 & 0.24985 & -0.34246 & 0.24978 & 0.05453 \\
\hline Spiders & Beta & MFS & -0.54204 & 0.20134 & -0.53554 & 0.20897 & 0.04400 \\
\hline Spiders & Beta & SHDI & 0.00123 & 0.19900 & 0.12427 & 0.20847 & 0.09643 \\
\hline Spiders & Beta & Year & -1.11445 & 0.33545 & -1.08243 & 0.34432 & 0.04296 \\
\hline Spiders & Alpha & Intercept & 2.41241 & 0.19489 & 2.39173 & 0.19699 & 0.01888 \\
\hline Spiders & Alpha & P.AG & -0.39603 & 0.18484 & -0.40653 & 0.18027 & 0.02541 \\
\hline Spiders & Alpha & MFS & -0.33300 & 0.19370 & -0.29515 & 0.20391 & 0.04853 \\
\hline Spiders & Alpha & SHDI & -0.05105 & 0.16627 & 0.06496 & 0.16500 & 0.08895 \\
\hline Spiders & Alpha & Year & -0.52863 & 0.29668 & -0.48925 & 0.30165 & 0.03561 \\
\hline Spiders & Abund. & Intercept & 19.96763 & 2.27490 & 19.59977 & 2.29466 & 0.21906 \\
\hline Spiders & Abund. & P.AG & -5.00481 & 2.27496 & -5.36364 & 2.16738 & 0.26287 \\
\hline Spiders & Abund. & MFS & -4.31533 & 2.36360 & -3.67747 & 2.46810 & 0.52831 \\
\hline Spiders & Abund. & SHDI & -0.90394 & 1.98015 & 0.67792 & 1.93983 & 1.07552 \\
\hline Spiders & Abund. & Year & -1.37658 & 3.48382 & -0.67644 & 3.53090 & 0.41339 \\
\hline Syrphids & Gamma & Intercept & 3.21300 & 0.28833 & 3.17394 & 0.28929 & 0.02629 \\
\hline Syrphids & Gamma & P.AG & 0.25698 & 0.32194 & 0.12624 & 0.32341 & 0.05168 \\
\hline Syrphids & Gamma & MFS & -0.52562 & 0.25192 & -0.42930 & 0.25332 & 0.04672 \\
\hline Syrphids & Gamma & SHDI & 0.01870 & 0.25314 & 0.24027 & 0.24915 & 0.13354 \\
\hline Syrphids & Gamma & Year & -1.98513 & 0.42058 & -1.91107 & 0.42204 & 0.05014 \\
\hline Syrphids & Beta & Intercept & 1.81542 & 0.16990 & 1.79617 & 0.17029 & 0.01259 \\
\hline Syrphids & Beta & P.AG & 0.12539 & 0.19175 & 0.05812 & 0.18849 & 0.02805 \\
\hline Syrphids & Beta & MFS & -0.27943 & 0.14678 & -0.23658 & 0.14609 & 0.02322 \\
\hline Syrphids & Beta & SHDI & 0.00673 & 0.14807 & 0.11399 & 0.14622 & 0.07465 \\
\hline Syrphids & Beta & Year & -1.06692 & 0.24709 & -1.03040 & 0.24778 & 0.02401 \\
\hline Syrphids & Alpha & Intercept & 1.39505 & 0.13350 & 1.37562 & 0.13427 & 0.01438 \\
\hline Syrphids & Alpha & P.AG & 0.11307 & 0.14945 & 0.05310 & 0.14820 & 0.02379 \\
\hline Syrphids & Alpha & MFS & -0.24301 & 0.11556 & -0.19037 & 0.11911 & 0.02603 \\
\hline Syrphids & Alpha & SHDI & 0.02440 & 0.11701 & 0.13126 & 0.11343 & 0.06328 \\
\hline Syrphids & Alpha & Year & -0.91343 & 0.19440 & -0.87657 & 0.19591 & 0.02743 \\
\hline Syrphids & Abund. & Intercept & 11.54142 & 1.47406 & 11.49677 & 1.49451 & 0.09352 \\
\hline Syrphids & Abund. & P.AG & -1.54393 & 1.35576 & -1.58651 & 1.29394 & 0.13634 \\
\hline Syrphids & Abund. & MFS & -1.98431 & 1.22445 & -1.56737 & 1.26497 & 0.24762 \\
\hline Syrphids & Abund. & SHDI & -0.88370 & 1.23723 & -0.23130 & 1.25786 & 0.71613 \\
\hline Syrphids & Abund. & Year & -7.79159 & 2.15908 & -7.70985 & 2.20481 & 0.17882 \\
\hline
\end{tabular}


Table M.5 Model parameter details for the beetles, birds, and butterflies taxa.

Simulated values are based on landscape-grouped simulation.

\begin{tabular}{|c|c|c|c|c|c|c|c|}
\hline Taxon & Diversity & Variable & $\widehat{\bar{\theta}}_{\text {hard }}$ & $\widehat{\operatorname{se}}\left(\widehat{\bar{\Theta}}_{\text {hard }}\right)$ & $\widehat{\bar{\Theta}}_{\text {sim }}$ & $\widehat{\operatorname{se}}\left(\widehat{\widehat{\theta}}_{\operatorname{sim}}\right)$ & $\sigma_{\text {sim }}$ \\
\hline Beetles & Gamma & Intercept & 5.63344 & 0.43545 & 5.59720 & 0.43631 & 0.02849 \\
\hline Beetles & Gamma & P.AG & -0.94083 & 0.38060 & -0.98779 & 0.37833 & 0.03571 \\
\hline Beetles & Gamma & MFS & -0.57537 & 0.45022 & -0.50051 & 0.47598 & 0.10352 \\
\hline Beetles & Gamma & SHDI & 0.26849 & 0.33173 & 0.21887 & 0.33466 & 0.14895 \\
\hline Beetles & Gamma & Year & 0.70900 & 0.66218 & 0.77923 & 0.66490 & 0.05408 \\
\hline Beetles & Beta & Intercept & 2.70876 & 0.26747 & 2.68676 & 0.26698 & 0.01688 \\
\hline Beetles & Beta & P.AG & -0.70850 & 0.22644 & -0.73823 & 0.22730 & 0.02035 \\
\hline Beetles & Beta & MFS & -0.23258 & 0.30386 & -0.17427 & 0.31240 & 0.07721 \\
\hline Beetles & Beta & SHDI & 0.24326 & 0.19464 & 0.21569 & 0.19714 & 0.07931 \\
\hline Beetles & Beta & Year & 0.31103 & 0.40468 & 0.35354 & 0.40424 & 0.03220 \\
\hline Beetles & Alpha & Intercept & 2.91601 & 0.18774 & 2.90952 & 0.18970 & 0.00892 \\
\hline Beetles & Alpha & P.AG & -0.23184 & 0.17738 & -0.24612 & 0.17759 & 0.02014 \\
\hline Beetles & Alpha & MFS & -0.27167 & 0.15666 & -0.26258 & 0.16640 & 0.03155 \\
\hline Beetles & Alpha & SHDI & 0.01861 & 0.15915 & -0.00376 & 0.16086 & 0.08522 \\
\hline Beetles & Alpha & Year & 0.41465 & 0.27863 & 0.42755 & 0.28339 & 0.01643 \\
\hline Beetles & Abund. & Intercept & 101.83312 & 19.00459 & 102.03481 & 19.07818 & 0.72798 \\
\hline Beetles & Abund. & P.AG & 1.67640 & 21.51735 & 3.58631 & 21.68426 & 3.61472 \\
\hline Beetles & Abund. & MFS & 30.99761 & 15.72543 & 30.40614 & 16.08242 & 2.57384 \\
\hline Beetles & Abund. & SHDI & -14.69760 & 16.39867 & -12.72417 & 16.70143 & 7.17488 \\
\hline Beetles & Abund. & Year & 31.11234 & 27.69229 & 30.67069 & 27.87287 & 1.30773 \\
\hline Birds & Gamma & Intercept & 6.40541 & 0.39325 & 6.42426 & 0.39616 & 0.02340 \\
\hline Birds & Gamma & P.AG & -0.76422 & 0.33225 & -0.71474 & 0.35009 & 0.03549 \\
\hline Birds & Gamma & MFS & -0.43971 & 0.38512 & -0.50817 & 0.37869 & 0.06947 \\
\hline Birds & Gamma & SHDI & 0.12662 & 0.29868 & 0.12200 & 0.31033 & 0.15336 \\
\hline Birds & Gamma & Year & 0.53066 & 0.59452 & 0.49459 & 0.60129 & 0.04611 \\
\hline Birds & Beta & Intercept & 3.88851 & 0.26905 & 3.90518 & 0.27002 & 0.01875 \\
\hline Birds & Beta & P.AG & -0.53457 & 0.24179 & -0.48871 & 0.25694 & 0.03062 \\
\hline Birds & Beta & MFS & -0.38916 & 0.25831 & -0.43470 & 0.25249 & 0.04548 \\
\hline Birds & Beta & SHDI & 0.09358 & 0.21054 & 0.08575 & 0.21799 & 0.11163 \\
\hline Birds & Beta & Year & 0.54993 & 0.40868 & 0.51793 & 0.41086 & 0.03709 \\
\hline Birds & Alpha & Intercept & 2.53272 & 0.15160 & 2.53483 & 0.15216 & 0.00588 \\
\hline Birds & Alpha & P.AG & -0.21466 & 0.12289 & -0.20641 & 0.12596 & 0.01108 \\
\hline Birds & Alpha & MFS & -0.06689 & 0.14350 & -0.08491 & 0.14331 & 0.02575 \\
\hline Birds & Alpha & SHDI & 0.02701 & 0.11199 & 0.02913 & 0.11444 & 0.05403 \\
\hline Birds & Alpha & Year & -0.05023 & 0.22689 & -0.05417 & 0.22825 & 0.01151 \\
\hline Birds & Abund. & Intercept & 16.21070 & 1.01088 & 16.19815 & 1.01032 & 0.03348 \\
\hline Birds & Abund. & P.AG & -1.14229 & 0.84596 & -1.10823 & 0.88155 & 0.08015 \\
\hline Birds & Abund. & MFS & -0.78653 & 0.85855 & -0.92511 & 0.86452 & 0.14207 \\
\hline Birds & Abund. & SHDI & 0.19461 & 0.77073 & 0.28421 & 0.79084 & 0.38181 \\
\hline Birds & Abund. & Year & -1.76146 & 1.49843 & -1.73682 & 1.49956 & 0.06653 \\
\hline Butterflies & Gamma & Intercept & 3.81163 & 0.42486 & 3.79346 & 0.42755 & 0.01836 \\
\hline Butterflies & Gamma & P.AG & 0.10302 & 0.45725 & 0.07834 & 0.47834 & 0.08454 \\
\hline Butterflies & Gamma & MFS & -1.29118 & 0.35185 & -1.23098 & 0.35015 & 0.05516 \\
\hline Butterflies & Gamma & SHDI & -0.33009 & 0.35154 & -0.13481 & 0.36728 & 0.19116 \\
\hline Butterflies & Gamma & Year & 0.60316 & 0.60901 & 0.63605 & 0.61346 & 0.03513 \\
\hline Butterflies & Beta & Intercept & 2.05608 & 0.24387 & 2.04154 & 0.24417 & 0.01453 \\
\hline Butterflies & Beta & P.AG & 0.14291 & 0.25833 & 0.12254 & 0.26657 & 0.04833 \\
\hline Butterflies & Beta & MFS & -0.78570 & 0.21606 & -0.74833 & 0.21026 & 0.04415 \\
\hline Butterflies & Beta & SHDI & -0.26052 & 0.19911 & -0.16604 & 0.20617 & 0.10660 \\
\hline Butterflies & Beta & Year & 0.36212 & 0.35259 & 0.38857 & 0.35214 & 0.02774 \\
\hline Butterflies & Alpha & Intercept & 1.75920 & 0.21107 & 1.75121 & 0.21353 & 0.00904 \\
\hline Butterflies & Alpha & P.AG & -0.05955 & 0.24160 & -0.07652 & 0.24960 & 0.04948 \\
\hline Butterflies & Alpha & MFS & -0.52007 & 0.16660 & -0.49159 & 0.17094 & 0.02472 \\
\hline Butterflies & Alpha & SHDI & -0.06150 & 0.18073 & 0.04909 & 0.18892 & 0.10057 \\
\hline Butterflies & Alpha & Year & 0.23415 & 0.30229 & 0.24880 & 0.30753 & 0.01723 \\
\hline Butterflies & Abund. & Intercept & 16.90563 & 2.88286 & 16.74232 & 2.89699 & 0.28200 \\
\hline Butterflies & Abund. & P.AG & 2.62098 & 3.18174 & 2.15245 & 3.27744 & 0.73159 \\
\hline Butterflies & Abund. & MFS & -5.80114 & 2.50810 & -5.01952 & 2.70462 & 0.69733 \\
\hline Butterflies & Abund. & SHDI & 1.52726 & 2.52047 & 3.12553 & 2.42729 & 1.45390 \\
\hline Butterflies & Abund. & Year & 1.28218 & 4.20153 & 1.58431 & 4.23336 & 0.53414 \\
\hline
\end{tabular}


Table M.6 Model parameter details for the plants, spiders, and syrphids taxa.

Simulated values are based on landscape-grouped simulation.

\begin{tabular}{|c|c|c|c|c|c|c|c|}
\hline Taxon & Diversity & Variable & $\widehat{\bar{\Theta}}_{\text {hard }}$ & $\widehat{\operatorname{se}}\left(\widehat{\bar{\theta}}_{\text {hard }}\right)$ & $\widehat{\bar{\theta}}_{\text {sim }}$ & $\widehat{\operatorname{se}}\left(\widehat{\bar{\theta}}_{\operatorname{sim}}\right)$ & $\sigma_{\mathrm{sim}}$ \\
\hline Plants & Gamma & Intercept & 36.93728 & 1.74601 & 36.92857 & 1.72440 & 0.08083 \\
\hline Plants & Gamma & P.AG & -1.50311 & 1.82609 & -0.96273 & 1.85834 & 0.34024 \\
\hline Plants & Gamma & MFS & -7.14049 & 1.48271 & -7.41794 & 1.45010 & 0.21579 \\
\hline Plants & Gamma & SHDI & 0.12493 & 1.46245 & 0.02851 & 1.46548 & 0.66096 \\
\hline Plants & Gamma & Year & -11.36525 & 2.54787 & -11.34802 & 2.50618 & 0.15993 \\
\hline Plants & Beta & Intercept & 21.83327 & 1.06045 & 21.82914 & 1.05195 & 0.05227 \\
\hline Plants & Beta & P.AG & -0.38279 & 1.16016 & -0.01198 & 1.16392 & 0.22998 \\
\hline Plants & Beta & MFS & -3.86727 & 0.88465 & -4.01928 & 0.88645 & 0.15487 \\
\hline Plants & Beta & SHDI & 0.73247 & 0.89698 & 0.57710 & 0.89901 & 0.42494 \\
\hline Plants & Beta & Year & -6.48392 & 1.53658 & -6.47574 & 1.52383 & 0.10342 \\
\hline Plants & Alpha & Intercept & 15.03345 & 0.78950 & 15.04918 & 0.77692 & 0.05202 \\
\hline Plants & Alpha & P.AG & -1.16635 & 0.79198 & -0.97682 & 0.80869 & 0.15175 \\
\hline Plants & Alpha & MFS & -3.15056 & 0.77954 & -3.31562 & 0.74623 & 0.16340 \\
\hline Plants & Alpha & SHDI & -0.57426 & 0.63972 & -0.52322 & 0.64311 & 0.28758 \\
\hline Plants & Alpha & Year & -4.74171 & 1.18841 & -4.77284 & 1.15989 & 0.10293 \\
\hline Spiders & Gamma & Intercept & 5.16664 & 0.39490 & 5.14299 & 0.39791 & 0.04064 \\
\hline Spiders & Gamma & P.AG & -0.73537 & 0.42194 & -0.74063 & 0.42111 & 0.08453 \\
\hline Spiders & Gamma & MFS & -0.86561 & 0.37696 & -0.84479 & 0.38724 & 0.08777 \\
\hline Spiders & Gamma & SHDI & -0.05559 & 0.34756 & 0.11623 & 0.34051 & 0.19696 \\
\hline Spiders & Gamma & Year & -1.64768 & 0.59980 & -1.60172 & 0.60637 & 0.07566 \\
\hline Spiders & Beta & Intercept & 2.75176 & 0.22361 & 2.74410 & 0.22443 & 0.01898 \\
\hline Spiders & Beta & P.AG & -0.34707 & 0.24985 & -0.33368 & 0.25246 & 0.05444 \\
\hline Spiders & Beta & MFS & -0.54204 & 0.20134 & -0.54353 & 0.20470 & 0.04211 \\
\hline Spiders & Beta & SHDI & 0.00123 & 0.19900 & 0.07963 & 0.19833 & 0.10496 \\
\hline Spiders & Beta & Year & -1.11445 & 0.33545 & -1.09884 & 0.33733 & 0.03507 \\
\hline Spiders & Alpha & Intercept & 2.41241 & 0.19489 & 2.39687 & 0.19594 & 0.01760 \\
\hline Spiders & Alpha & P.AG & -0.39603 & 0.18484 & -0.40485 & 0.18217 & 0.02626 \\
\hline Spiders & Alpha & MFS & -0.33300 & 0.19370 & -0.30439 & 0.20122 & 0.04879 \\
\hline Spiders & Alpha & SHDI & -0.05105 & 0.16627 & 0.03361 & 0.15965 & 0.09827 \\
\hline Spiders & Alpha & Year & -0.52863 & 0.29668 & -0.49892 & 0.29909 & 0.03300 \\
\hline Spiders & Abund. & Intercept & 19.96763 & 2.27490 & 19.64822 & 2.28146 & 0.20551 \\
\hline Spiders & Abund. & P.AG & -5.00481 & 2.27496 & -5.34998 & 2.18534 & 0.27516 \\
\hline Spiders & Abund. & MFS & -4.31533 & 2.36360 & -3.74761 & 2.44665 & 0.54638 \\
\hline Spiders & Abund. & SHDI & -0.90394 & 1.98015 & 0.37914 & 1.86969 & 1.16987 \\
\hline Spiders & Abund. & Year & -1.37658 & 3.48382 & -0.76804 & 3.49904 & 0.38526 \\
\hline Syrphids & Gamma & Intercept & 3.21300 & 0.28833 & 3.19001 & 0.28729 & 0.01817 \\
\hline Syrphids & Gamma & P.AG & 0.25698 & 0.32194 & 0.13683 & 0.32682 & 0.05536 \\
\hline Syrphids & Gamma & MFS & -0.52562 & 0.25192 & -0.44271 & 0.24914 & 0.04502 \\
\hline Syrphids & Gamma & SHDI & 0.01870 & 0.25314 & 0.17527 & 0.24267 & 0.14463 \\
\hline Syrphids & Gamma & Year & -1.98513 & 0.42058 & -1.94205 & 0.41673 & 0.03449 \\
\hline Syrphids & Beta & Intercept & 1.81542 & 0.16990 & 1.80294 & 0.16921 & 0.00918 \\
\hline Syrphids & Beta & P.AG & 0.12539 & 0.19175 & 0.06129 & 0.18999 & 0.03013 \\
\hline Syrphids & Beta & MFS & -0.27943 & 0.14678 & -0.24120 & 0.14450 & 0.02400 \\
\hline Syrphids & Beta & SHDI & 0.00673 & 0.14807 & 0.08521 & 0.14173 & 0.08143 \\
\hline Syrphids & Beta & Year & -1.06692 & 0.24709 & -1.04347 & 0.24496 & 0.01743 \\
\hline Syrphids & Alpha & Intercept & 1.39505 & 0.13350 & 1.38539 & 0.13332 & 0.00977 \\
\hline Syrphids & Alpha & P.AG & 0.11307 & 0.14945 & 0.05959 & 0.15068 & 0.02549 \\
\hline Syrphids & Alpha & MFS & -0.24301 & 0.11556 & -0.19954 & 0.11633 & 0.02364 \\
\hline Syrphids & Alpha & SHDI & 0.02440 & 0.11701 & 0.09611 & 0.11138 & 0.06898 \\
\hline Syrphids & Alpha & Year & -0.91343 & 0.19440 & -0.89541 & 0.19329 & 0.01853 \\
\hline Syrphids & Abund. & Intercept & 11.54142 & 1.47406 & 11.50178 & 1.48719 & 0.08875 \\
\hline Syrphids & Abund. & P.AG & -1.54393 & 1.35576 & -1.57973 & 1.30186 & 0.14727 \\
\hline Syrphids & Abund. & MFS & -1.98431 & 1.22445 & -1.59758 & 1.25584 & 0.28255 \\
\hline Syrphids & Abund. & SHDI & -0.88370 & 1.23723 & -0.35978 & 1.20436 & 0.76087 \\
\hline Syrphids & Abund. & Year & -7.79159 & 2.15908 & -7.71900 & 2.18698 & 0.16897 \\
\hline
\end{tabular}

